\title{
Antidepressants, serotonin and cognition : applied and fundamental studies in human volunteers
}

Citation for published version (APA):

Wingen, M. (2007). Antidepressants, serotonin and cognition : applied and fundamental studies in human volunteers. [Doctoral Thesis, Maastricht University]. https://doi.org/10.26481/dis.20070524mw

Document status and date:

Published: 01/01/2007

DOI:

10.26481/dis.20070524mw

Document Version:

Publisher's PDF, also known as Version of record

\section{Please check the document version of this publication:}

- A submitted manuscript is the version of the article upon submission and before peer-review. There can be important differences between the submitted version and the official published version of record.

People interested in the research are advised to contact the author for the final version of the publication, or visit the DOI to the publisher's website.

- The final author version and the galley proof are versions of the publication after peer review.

- The final published version features the final layout of the paper including the volume, issue and page numbers.

Link to publication

\footnotetext{
General rights rights.

- You may freely distribute the URL identifying the publication in the public portal. please follow below link for the End User Agreement:

www.umlib.nl/taverne-license

Take down policy

If you believe that this document breaches copyright please contact us at:

repository@maastrichtuniversity.nl

providing details and we will investigate your claim.
}

Copyright and moral rights for the publications made accessible in the public portal are retained by the authors and/or other copyright owners and it is a condition of accessing publications that users recognise and abide by the legal requirements associated with these

- Users may download and print one copy of any publication from the public portal for the purpose of private study or research.

- You may not further distribute the material or use it for any profit-making activity or commercial gain

If the publication is distributed under the terms of Article $25 \mathrm{fa}$ of the Dutch Copyright Act, indicated by the "Taverne" license above, 


\section{Antidepressants, serotonin and cognition Applied and fundamental studies in human volunteers}




\section{Paranimfen}

Kim Kuypers

Inez Ramakers

(C) M. Wingen, Maastricht 2007

Printed by: Schrijen Lippertz Huntjens BV, Voerendaal ISBN: 978-90-9021691-1 


\section{Antidepressants, serotonin and cognition Applied and fundamental studies in human volunteers}

\section{Proefschrift}

ter verkrijging van de graad van doctor aan de Universiteit Maastricht, op gezag van de rector Magnificus, Prof. mr. G.P.M.F. Mols, volgens het besluit van het College van Decanen, in het openbaar te verdedigen op donderdag 24 mei 2007 om 12:00 uur

door

Marleen Wingen

Geboren op 28-08-1980 te Heerlen 


\section{Promotor}

Prof. dr. W.J. Riedel

\section{Copromotor}

Dr. J.G. Ramaekers

\section{Beoordelingscommissie}

Prof. dr. R. Goebel

Prof. dr. J. Jolles (voorzitter)

Prof. dr. B. Leonard (National University of Ireland)

Dr. J. Prickaerts

Prof. dr. W. Sturm (UK-Aachen)

The research described in this thesis was conducted at the Brain \& Behavior Institute, the department of Neurocognition, Maastricht University. Two studies (chapter 3 and 5) described in this thesis were funded by Lunbeck Denmark BV. Additional financial support was received from the EU $5^{\text {th }}$ Framework Programme and Hersenstichting Nederland (chapter 4).

Financial support for the publication of this thesis was kindly provided by Lundbeck BV, NV Organon, Wyeth Pharmaceuticals BV, Ministerie van Verkeer en Waterstaat, AstraZeneca BV, Servier Nederland Farma BV. 


\section{Contents}

Chapter 1 General Introduction $\quad 7$

Chapter 2 Serotonin and human cognitive performance $\quad 17$

Chapter 3 Actual driving performance and psychomotor function in healthy subjects after acute and subchronic treatment with escitalopram, mirtazapine and placebo

Chapter 4 Driving impairment in depressed patients receiving long-term antidepressant treatment

Chapter 5 Verbal memory performance during subchronic challenge with a selective serotonergic and a mixed action antidepressant

Chapter 6 Selective verbal and spatial memory impairment after 5- $\mathrm{HT}_{1 \mathrm{~A}}$ and 5-HT $2 \mathrm{~A}$ receptor blockade $n$ healthy volunteers pre-treated with an SSRI

Chapter 7 The role of 5- $\mathrm{HT}_{1 \mathrm{~A}}$ and 5- $\mathrm{HT}_{2 \mathrm{~A}}$ receptors in attention and motor control: a mechanistic study in healthy volunteers $\quad 97$

Chapter 8 Sustained attention and serotonin: a pharmaco-fMRI study $\quad 113$

$\begin{array}{ll}\text { Chapter } 9 \text { General discussion } & 127\end{array}$

$\begin{array}{ll}\text { References } & 137\end{array}$

$\begin{array}{ll}\text { Summary } & 161\end{array}$

$\begin{array}{ll}\text { Samenvatting } & 167\end{array}$

Dankwoord 173

Curriculum Vitae 175

$\begin{array}{ll}\text { Publications } & 175\end{array}$ 

Chapter 1

General Introduction

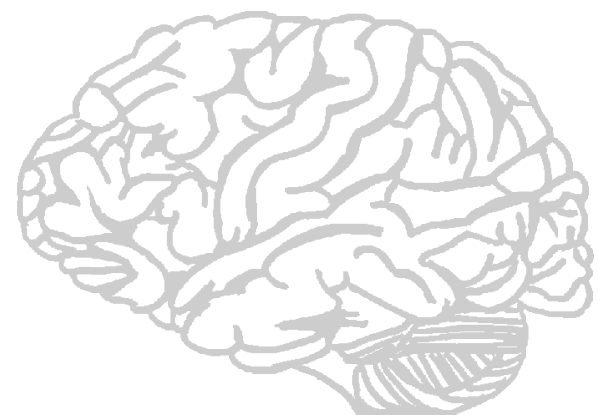




\section{Behavioral toxicity of antidepressants}

Adverse effects of antidepressants will be represented in daily life by impaired cognitive performance and car driving. The use of antidepressants sometimes causes people to get drowsy or slow down and this may cause accidents on the road or during work. This reversible pharmacological effect of antidepressants or drugs in general is called behavioural toxicity and causes disruption of neuropsychological processes and inhibits or reduces the efficiency of normal behaviour (Ramaekers, 1998). The seriousness, appearance and the type of adverse events differ between antidepressants, depending on the mechanism of action. In general, antidepressants with anticholinergic or sedative actions impair cognitive function (Amado-Boccara et al., 1995). Experimental psychopharmacological research in the present thesis focuses on the behavioural toxicity of antidepressants. There are two different aims underlying the various experiments. Firstly, in applied research the side effects of specific antidepressant treatments on cognition and driving performance are described. Secondly, the role of the neurotransmitter serotonin in performance is assessed by experimentally manipulating antidepressant treatment.

A typical experiment described in this thesis, is conducted according to a within subject design. The behavioural adverse effects of a drug of interest are evaluated by comparing the drug to a placebo treatment while cognitive and psychomotor tests are conducted as outcome measures. An active control condition is often included to compare the effect of the drug of interest quantitatively to another drug of which the behavioural adverse effects are already established in previous research. Cognition and psychomotor functioning are usually assessed in the laboratory by means of computerized cognitive tasks. In the present thesis, various computerized tasks are used covering a broad range of cognitive functions. Assessing the effects of a drug on driving performance is an important issue in experimental psychopharmacological research. The knowledge of drug effects on cognitive functions obtained using abstract computerized laboratory tasks of cognition, is translated to predict drug effects on car driving and other daily life functions. In addition to laboratory tasks that measure different aspects of driving related behaviour as attention, this thesis also contains descriptions of studies of drug effects on actual driving performance on the road.

In general, both types of psychopharmacological studies can be conducted in healthy volunteers as well as in mentally ill patients. Examining the possible side effects on cognition or driving performance of antidepressant medication in healthy 
volunteers will give a clear look at the specific side effects independent of any effect of mental illness itself. On the other hand, given that depressed patients use these medications in daily life, it is important to know what the net effect is on cognition and driving performance in patients. Due to symptoms of the psychiatric illness and the interaction between medication use and the symptoms, cognitive functioning of patients is possibly different in- and develops differently over time when compared to healthy volunteers (Lane \& O'Hanlon, 1999). In the present thesis, applied research into over-the-road driving performance in healthy as well as depressed persons is assessed.

\section{Serotonin and cognition}

Serotonin or 5-hydroxytryptamine (5-HT) is an essential monoamine neurotransmitter involved in behavioural functions including mood, sleep, aggression, appetite and sex (Aghajanian \& Sanders-Bush, 2002). Serotonin is released from the raphe nuclei located in the brainstem and it follows 5 major serotonergic pathways projecting to the brain and the body via the central- and peripheral nervous system respectively. Only $5 \%$ of the total serotonin transmission is distributed in the brain, the most amount of serotonin ( $80 \%$ of body $5-\mathrm{HT})$ is found in the gastrointestinal tract. In the brain, projections lead to different areas such as prefrontal cortex, basal ganglia, hippocampus and hypothalamus (Stahl, 1998b). A wide variety of serotonergic receptor groups and subtypes exist. For example the group of $5-\mathrm{HT}_{1}$ receptors includes the $5-\mathrm{HT}_{1 \mathrm{~A}}$ somatodendritic autoreceptor mainly situated in the hippocampus, amygdala and dorsal raphe nucleus and the group of $5-\mathrm{HT}_{2}$ receptors includes the $5-\mathrm{HT}_{2 \mathrm{~A}}$ receptor largely available in the cerebral cortex (Buhot et al., 2000). One mechanism to eliminate serotonin from the brain is reuptake of serotonin from the synaptic cleft.

Research in animals as well as in humans demonstrated that serotonin is involved in different aspects of cognition including attention and memory (Buhot et al., 2000; Matrenza et al., 2004; Meneses, 1999; Riedel et al., 1999). Manipulating or challenging the serotonergic system in humans is only possible through indirect mechanisms as serotonin itself does not cross the blood brain barrier. Decreased serotonin availability can be accomplished experimentally by a method called tryptophan depletion in which the availability of the precursor of serotonin (tryptophan) is diminished. This leads to a reduction of serotonin synthesis (Moore et al., 2000). Increased serotonin levels may be obtained by blocking the reuptake 
mechanisms through oral administration of selective serotonin reuptake inhibitors (SSRIs). Acute experimentally decreased serotonin levels has previously been shown to impair memory consolidation on a verbal memory task (Riedel, Klaassen et al., 1999; Schmitt et al., 2000) while conversely, another study showed improved delayed memory recall after intravenous SSRI administration (Harmer et al., 2002). In addition, SSRIs have been shown to impair sustained attention in several studies, measured with the Mackworth Clock Test (O'Hanlon et al., 1998; Ramaekers et al., 1995; Riedel et al., 2005; Schmitt, Ramaekers et al., 2002). Furthermore, acute tryptophan depletion caused improvement of selective attention (Coull et al., 1995; Schmitt et al., 2000). The question arises what pharmacological mechanisms underlie these effects of serotonin on memory and attention. Consequently, it is interesting to examine these fundamental mechanisms on the receptor level or on different brain mechanisms and interacting neurotransmitters.

In the present thesis, the role of acutely increased serotonin levels in memory and attention in humans has been investigated one step further by exploring different brain and receptor mechanisms (i.e. 5- $\mathrm{HT}_{1 \mathrm{~A}}$ and 5- $\mathrm{HT}_{2 \mathrm{~A}}$ receptors) using selective serotonin reuptake inhibition. To increase serotonin, the senantiomer of the SSRI citalopram has been used alone or in combination with receptor antagonists. Escitalopram is the most selective and most potent antidepressant among the group of selective serotonin reuptake inhibitors and it serves as a valuable tool in challenging the serotonergic system.

\section{Depression, antidepressants, cognition and driving performance}

Serotonergic dysfunctioning is assumed to be part of the etiology of a variety of psychiatric disorders including major depressive disorder (Charney, 1998; Meltzer, 1989). Major depressive disorder is characterized by depressed mood, loss of interest and serious loss of energy. Furthermore, patients can experience abnormal sleep and psychomotor agitation or slowing. According to the World Health Organization, depression is a common mental disorder with severe consequences for the patient and for the society. It causes the second highest disease burden in the age group 15-44 and by the year 2020 it will be the second highest ranking disease burden in the developed world (WHO, 2001).

In addition to the more commonly recognised features, depression is often accompanied by a decline in cognitive and psychomotor functioning. The cognitive deficits are related to the mental illness itself and not to more general physical 
disease-related factors (e.g. stress or not feeling well) (Den Hartog et al., 2003). An association between depression and impairment of memory has previously been demonstrated (Burt et al., 1995). Especially, problems with encoding and retrieval from episodic memory (Brand et al., 1992; Den Hartog et al., 2003; Zakzanis et al., 1998) and deficits in attention can be seen in depression (Landro et al., 2001; Purcell et al., 1997). A review concerning cognitive deficits in depression (Austin et al., 2001) showed impairment in episodic memory (verbal and visual) and impairment in executive deficits, for example difficulties on measures of verbal fluency and attentional set-shifting. Consequently, it is expected that the quality of daily living functions such as car driving is impaired in depressed patients and moreover, traffic safety is compromised.

In the Netherlands, around $45 \%$ of the depressive population receives professional care and around $42 \%$ of the depressive population is treated with an antidepressant medication (Spijker et al., 2001). Based on information obtained from Dutch pharmacies, antidepressants are among the most prescribed drugs and 9 of 10 prescriptions are repeat prescriptions. The number of registered antidepressant prescriptions was 5,5 million in 2005 accounting for a cost to the society of 162 million euros (Stichting Farmaceutische Kengetallen, 2006). Antidepressants can be classified by their mechanism of action, for example some inhibit the enzyme monoamine oxidase and thereby increase the availability of the monoamines (MAO inhibitors e.g. phenelzine and moclobemide) others block the reuptake of serotonin, norepinephrine and even dopamine (tricyclic antidepressants, TCAs e.g. amitriptyline and desipramine) (Stahl, 1998a). Paroxetine and citalopram are the two most prescribed antidepressants in the Netherlands; these drugs are typically classified as selective serotonin reuptake inhibitors (SSRIs). The therapeutic effect of SSRIs is thought to be based on $5-\mathrm{HT}_{1 \mathrm{~A}}$ autoreceptor desensitization. Somatodendritic $5-\mathrm{HT}_{1 \mathrm{~A}}$ autoreceptors act to reduce serotonin neuronal firing rates by means of a feedback mechanism. During prolonged SSRI treatment, the autoreceptor will desensitize and thereby neuronal firing will be disinhibited. Consequently, serotonin neurotransmission is increased (Stahl, 1998b). The third most commonly used antidepressant is venlafaxine as a serotonergic and noradrenergic reuptake inhibitor (SNRI) (Stichting Farmaceutische Kengetallen, 2006).

Depending on the mechanism of action, antidepressants exert different side effects, physically as well as cognitively. MAO inhibitors have serious side effects 
including elevation of blood pressure. The use of TCAs is accompanied by side effects including constipation, blurred vision, dry mouth and memory impairment mediated by muscarinic anticholinergic action and dizziness, drowsiness and sedation mediated by a high affinity for $\mathrm{H}_{1}$ histaminic receptors or noradrenergic $\mathrm{a}_{1}$ receptors (Amado-Boccara et al., 1995; Stahl, 1998a). SSRIs are said to be better tolerated because they are free from negative cognitive and psychomotor effects compared to TCAs (Hindmarch, 1995). Nevertheless, SSRIs have different pharmacodynamic profiles compared to each other and therefore SSRIs may have different, effects on cognition and different adverse effects including nausea, sexual dysfunction and insomnia (Stahl, 1998b). In their therapeutic action, SSRIs all block the serotonin reuptake pump, but next to that SSRIs may differ in their binding to serotonin receptors, in anticholinergic effects, in effects on dopamine neurotransmission, in affinity for $\mathrm{H}_{1}$ receptors and in affinity for sigma1 binding site (Lane \& O'Hanlon, 1999). Cyclic antidepressants were shown to increase the relative risk of depressed patients to be involved in a traffic accident (Leveille et al., 1994; Ray et al., 1992) and to produce driving impairment through the first two weeks of administration in healthy volunteers (Ramaekers, 2003). Since depression by itself is associated with cognitive and psychomotor dysfunction, antidepressants with relatively less negative side effects or even positive effects on cognition are preferable.

The present thesis explored the side effects of commonly used antidepressants on cognition and actual driving performance in healthy volunteers as well as in depressed patients. Central to all chapters is the hypothesis that the core serotonergic action of antidepressants has specific effects (negative, neutral or positive) on cognition whereas other pharmacological mechanisms encountered in antidepressants, such as anicholinergic-, anti-adrenergic and antihistaminergic mechanisms may have a negative impact on performance.

\section{Aim and outline of the dissertation}

The aim of this thesis is on the one hand to give more information on the side effects of commonly used antidepressants on cognition and driving performance in healthy persons as well as patients and on the other hand to explore the role of serotonin in cognition, particularly memory and attention, manipulated at the receptor level by administering drugs to healthy volunteers and measured at the neuronal network level by means of fMRI and relating this to drug effects on cognitive performance. 
In Chapter 2 a general overview is given concerning evidence for a role of serotonin in human cognition discussing studies with healthy volunteers (e.g. using serotonergic manipulations as SSRIs and ATD) and with selected (clinical) populations (e.g. depressed patients and patients with Alzheimer's disease).

Chapter 3 and Chapter 4 are both applied studies investigating driving performance and side effects of antidepressants. In both studies, actual driving performance was assessed by two standard on the road driving tests and cognitive and psychomotor functions were measured by means of laboratory tasks. Chapter 3 is together with Chapter 5 derived from a large experiment; both chapters however address a specific aim.

In Chapter 3 it is specifically examined what the acute and subchronic effects of an SSRI (escitalopram) and a noradreneric and serotonergic antidepressant (mirtazapine) on driving performance and psychomotor function in healthy volunteers, in a randomized within subject design including 18 healthy volunteers. SSRIs are presumed to have few negative effects on car driving compared to TCA's. Citalopram, the parent compound of escitalopram is free from detrimental effects on psychomotor function (Fairweather et al., 1997; Herberg, 2001; Nathan et al., 2000). The effect of escitalopram on psychomotor function and actual driving performance had not yet been examined. Mirtazapine on the other hand was shown to impair psychomotor function and actual car driving in previous studies (Mattila et al., 1989; Ramaekers et al., 1998; Ridout et al., 2003). After evening dosages of escitalopram, mirtazapine and placebo on separate days for a period of 15 days, performance was measured the next day (day 2), at day 9 and at day 16. Day 2, 9 and 16 reflect performance after acute treatment, dose increase and steady state respectively.

It was hypothesized that acute and subchronic treatment with a selective serotonergic antidepressant would not affect actual driving performance and psychomotor function. In addition, it was expected that a mixed-action antidepressant, such as mirtazapine, would impair driving performance and psychomotor function by blocking central $\mathrm{H}_{1}$ receptors in the brain.

In Chapter 4 the actual driving performance and cognition in 24 depressed patients receiving long-term antidepressant (SSRI and SNRI) treatment compared to 24 
healthy volunteers in a between subject design is examined. Previous experimental studies on the effects of antidepressants on driving performance are mainly conducted in healthy volunteers rather than in depressed patients. In general, it is expected that antidepressants that are often prescribed nowadays such as SSRIs or SNRIs have few impairing effects on driving performance. Information on the longterm effects of antidepressant on driving performance of depressed patients was not available and had to be investigated. On a single test day, preceded by a training the day before, depressed patients receiving SSRI or SNRI treatment and matched healthy volunteers were tested on memory, attention and driving performance.

It was hypothesized that driving performance and cognition of depressed patients receiving long-term treatment with with serotonergic/noradrenergic antidepressants was comparable to that of matched healthy controls, because of alleviation of depressive symptoms caused by the non-sedating treatment

Chapter 5 to chapter 8 are challenge studies in which the role of serotonin in cognition was further explored by means of a selective antidepressant in healthy volunteers. Chapter 6 and 7 are both derived of a large study but these chapters are designed to address a specific aim. Different laboratory tasks were assessed to cover a variety of cognitive functions including different aspects of memory and attention, motor control and motor impulsivity.

Chapter 5 deals with serotonin and verbal memory by assessing the acute and subchronic effects of different dosages of escitalopram and mirtazapine on verbal memory measured in a randomized within subject-design with 18 healthy volunteers. In a previous study, the SSRI citalopram was used to manipulate serotonin and delayed verbal memory was improved in humans after an acute dose (Harmer et al., 2002). In the present study escitalopram was used as a selective tool to manipulate serotonin levels. No information on the effects of mirtazapine on verbal memory was available but by blocking the $\mathrm{H}_{1}$ receptor, mirtazapine has sedative properties (Anttila \& Leinonen, 2001). From days 1-7 an evening dose of escitalopram $10 \mathrm{mg}$, mirtazapine $30 \mathrm{mg}$ or placebo was administered and from days 8-15 an evening dose of escitalopram $20 \mathrm{mg}$, mirtazapine $45 \mathrm{mg}$ or placebo was administered. Verbal memory was assessed at the next day after the first evening of treatment administration (day 2), at day 9 and at day 16. 
It was hypothesized that an acute and subchronic dose of escitalopram may enhance verbal memory due to its selective serotonergic action and that acute and subchronic treatment with mirtazapine would impair verbal memory due to its histaminergic action

In Chapter 6 it is explored what the contribution of general serotonin levels and serotonergic receptor function (i.e. $5-\mathrm{HT}_{1 \mathrm{~A}}$ and $5-\mathrm{HT}_{2 \mathrm{~A}}$ receptors) on memory in a fundamental study including 16 healthy volunteers. It has been suggested that memory function may be mediated by serotonergic receptor activation (Kuypers \& Ramaekers, 2005) and candidate receptors are 5- $\mathrm{HT}_{1 \mathrm{~A}}$ and 5- $\mathrm{HT}_{2 \mathrm{~A}}$ receptors (Buhot et al., 2000; de Quervain et al., 2003; Meneses, 2002; Yasuno et al., 2003). On 4 separate test days, escitalopram $20 \mathrm{mg}$ was administered randomly in combination with pindolol $10 \mathrm{mg}$ (a very partial 5- $\mathrm{HT}_{1 \mathrm{~A}}$ receptor agonist), ketanserin $50 \mathrm{mg}$ (a 5$\mathrm{HT}_{2 \mathrm{~A}}$ receptor antagonist) or placebo. Several memory tasks were conducted including a verbal memory task, a spatial working memory task and a reversal learning task.

It was hypothesized that pindolol and ketanserin would augment the effect of escitalopram on memory by acutely elevating serotonin levels through blockade of the presynaptic 5-HT $\mathrm{T}_{1 \mathrm{~A}}$ autoreceptor and by decreasing dopamine function through blockade of the $5-\mathrm{HT}_{2 \mathrm{~A}}$ receptor respectively

Chapter 7 examines the involvement of general serotonin levels and serotonergic receptor function (i.e. 5- $\mathrm{HT}_{1 \mathrm{~A}}$ and 5- $\mathrm{HT}_{2 \mathrm{~A}}$ receptors) in motor control and attention in a within subject design with 16 healthy volunteers. It was shown before that SSRI administration has an impairing effect on sustained attention Test (O'Hanlon et al., 1998; Ramaekers et al., 1995; Riedel et al., 2005; Schmitt, Ramaekers et al., 2002). Possibly this effect is mediated by specific serotonergic receptor activation. On each test day (4 in total) tasks concerning attention and motor control were assessed including a sustained attention task, a selective attention task, a divided attention task, a critical tracking task and a stop signal task. Treatments on the test days were escitalopram $20 \mathrm{mg}$ in combination with pindolol $10 \mathrm{mg}$, ketanserin $50 \mathrm{mg}$ or placebo, all randomly assigned.

It was hypothesized that the SSRI escitalopram would enhance serotonergic levels and that

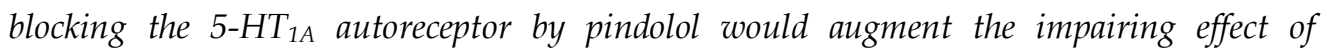


escitalopram on sustained attention. Furthermore, it was expected that blocking the 5-HT $\mathrm{T}_{2 \mathrm{~A}}$ receptor by ketanserin may decrease dopamine release and therefore may also augment the impairing effect of escitalopram on sustained attention.

Chapter 8 is a pharmaco-fMRI study in which the impairing role of serotonin on brain regions involved in sustained attention is investigated. Previous studies showed an impairment after SSRI administration on sustained attention measured with the Mackworth Clock Test (O'Hanlon et al., 1998; Ramaekers et al., 1995; Riedel et al., 2005; Schmitt, Ramaekers et al., 2002). The purpose of Chapter 8 was to investigate this impairing effect on the level of brain circuits associated with sustained attention in a balanced, double blind, 2-way crossover design. Escitalopram $20 \mathrm{mg}$ and placebo were administered at 2 different test days separated with a wash-out period for 7 days and participants performed the Mackworth Clock Test while lying in an fMRI headscanner.

It was hypothesized that increased serotonin levels after escitalopram administration would impair sustained attention and this was expected to be observed in brain regions involved in sustained attention

In the last chapter, Chapter 9, findings of the previous chapters are integrated and discussed in terms of whether they answered the research questions outlined above and implications for future studies are given. 


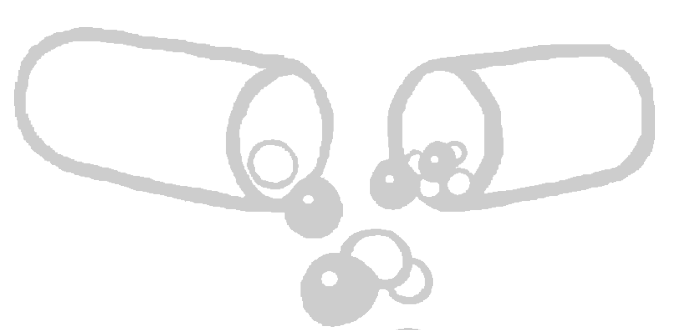

\title{
Chapter 2 Serotonin and human cognitive performance
}

\begin{abstract}
In the past decade, experimental studies involving healthy human volunteers have revealed that manipulations of the central serotonin (5-HT) system can produce quite specific changes in cognitive functioning, independent of overt mood changes. Reduced serotonin turnover is consistently associated with impaired long-term memory functioning. Low serotonin function may also impair cognitive flexibility and improve focused attention. On the other hand, stimulation of central serotonin has repeatedly been found to impair performance in a true vigilance task. Currently, there is little evidence for mirrored cognitive changes due to opposite serotonin manipulations in healthy volunteers. Given the mounting evidence for a role of serotonin in human cognition, reduced serotonin function could be directly linked to cognitive disturbances in certain conditions, such as in depression and Alzheimer's Disease (AD). There is evidence that stimulating (i.e. normalizing) serotonin activity in depression may have specific beneficial effects on cognition, independent of a general relief of depressive symptoms, but this premise needs to be confirmed by larger-scale clinical studies. Recently, a potential role of serotonin in the cognitive symptoms in $\mathrm{AD}$ has been identified, but there is insufficient data to evaluate the effects of serotonin stimulation on cognitive symptoms in AD. It is concluded that serotonin is a potential target for pharmacological cognition enhancement, particularly for restoration of impaired cognitive performance due to serotonin dysfunction. Further differentiation of the role of serotonin in normal and disturbed cognition and evaluation of the effects of serotonin manipulations in various populations is required to establish the full potential of serotonin drugs as cognition enhancers.
\end{abstract}

Schmitt, J. A. J., Wingen, M., Ramaekers, J. G., Evers, E. A. T., \& Riedel, W. J. (2006). Serotonin and human cognitive performance. Current Pharmaceutical Design, 12, 2473 2486. 


\section{Introduction}

Serotonin (5-HT) has long been implicated in a wide range of behavioural functions, specifically in mood regulation, aggression and impulsivity. Serotonergic disturbances are linked to a variety of psychopathological conditions, the most prominent being depression, bipolar disorder and obsessive compulsive disorder (Davis et al., 2002). In the past decade, experimental studies with animals and humans have revealed that serotonin may also play an important role in normal and disturbed cognitive functioning. The premise that serotonin is involved in cognitive functions has important implications. First, it broadens our understanding the origin of cognitive deficits that are associated with certain conditions. For example, suboptimal serotonin activity may be responsible to at least part of the cognitive deficits seen in depression (Riedel, Klaassen, \& Schmitt, 2002) Low serotonin has recently been related to cognitive deficits seen in Alzheimer's Disease (Lai et al., 2002; Newhouse et al., 2002; Porter et al., 2003) and may even contribute to age-related cognitive decline (Richter-Levin \& Segal, 1996). Even in healthy women with premenstrual complaints, cognitive deficits have been related to serotonergic disturbances (Bethea et al., 2002). Secondly, such insights provide the basis for the development of treatment strategies for such conditions. Currently, the serotonergic system is considered a promising target for cognition enhancement (Allain et al., 2003; Roth et al., 2004). Thirdly, knowledge of serotonin modulation of cognition helps predict and possibly avoid cognitive side effects of drugs affecting the serotonin system (Schmitt, 2001).

Several excellent reviews are available on animal studies on serotonergic modulation of cognition (Buhot, 1997; Buhot et al., 2000; Meneses, 1999; Robbins et al., 1998; Steckler \& Sahgal, 1995). Despite recent developments and insights, however, an extensive overview focusing on human studies in this field is not available. The aim of the current review is to discuss the evidence for a role of serotonin in human cognitive functions; identify specific human cognitive functions that may be mediated by serotonin; and to explore the conditions under which manipulations of central serotonergic activity may enhance cognitive performance in human subjects. For the purpose of this review, we will focus on the results from psychopharmacological manipulations in either healthy volunteers or selected patient groups. Although occasionally results from animal studies will be briefly addressed to provide supplementary information and identify possible neurobiological mechanisms, an extensive discussion of the animal data on serotonin and cognition is 
beyond the scope of this paper. For this, the reader is referred to the aforementioned review papers.

In humans, several psychopharmacological approaches can be employed to study serotonin function in relation to cognition. In recent years, acute tryptophan depletion (ATD) has gained popularity as a method to temporarily lower serotonin activity in the human brain. The method is based on reducing the availability of Ltryptophan (TRP), the only precursor for serotonin, in the brain. In short, ATD involves oral administration of an amino acid suspension without TRP, which reduces TRP transport into the brain by increasing competition for active transport sites across the blood-brain barrier. ATD leads to marked reductions of brain TRP, serotonin and 5-HIAA (for reviews see (Moore et al., 2000; Reilly et al., 1997)). For example, five hours after intake of the ATD mixture, the alfa-methyl-[11C]tryptophan labeled method of estimating serotonin synthesis rate yielded values across various regions throughout the brain suggesting that serotonin synthesis rate was reduced by $\sim 87 \%$ and $\sim 97 \%$ in healthy men and women, respectively (Nishizawa et al., 1997). In rats, ATD reduces spontaneous hippocampal serotonin release by approximately $40 \%$ (Fadda, 2000). In a similar fashion, increasing brain TRP availability through TRP loading can be used to stimulate central serotonin synthesis (Young, 1986; Young \& Leyton, 2002). A maximal increase in brain serotonin synthesis of $\sim 100 \%$ appears to be achieved after a $3 \mathrm{~g}$ oral TRP load (Young, 1996; Young \& Gauthier, 1981).

Quite a number of human studies have assessed cognitive changes after acute or sub-chronic administration of various medicinal drugs that stimulate serotonin activity throughout the brain. These are predominantly selective serotonin reuptake inhibitors (SSRIs) and various tricyclic antidepressants (TCAs), which increase serotonin neurotransmission by blocking the reuptake of serotonin into the presynaptic neuron and are widely prescribed as antidepressants. Their cognitive effects have been investigated in both healthy volunteers as well as various patient groups, particularly depressed patients. However, these studies are often clinical trials of antidepressant treatment efficacy in patients in which assessment of cognitive (side) effects is a secondary goal at most. Other studies were designed as head-tohead comparison of cognitive side effects of competing drugs in healthy volunteers. Because of lack of specificity for the serotonergic system, TCA trials are less suitable to study serotonin effects on cognition. Their anticholinergic, antithistaminergic, antidopaminergic and/or antinoradrenergic actions are known to adversely affect 
cognition (Amado-Boccara et al., 1995) and hence obscure any serotonin-related changes. For this reason, this review will focus on studies involving SSRIs, which lack significant affinities for non-serotonergic neurotransmitter systems, to evaluate the cognitive effects of global serotonin stimulation. In addition to the aforementioned methods to alter whole brain serotonin activity, a number of serotonin receptor agonists and antagonists with varying selectivity are available for human research. However, these compounds have only sporadically been used in human cognitive research. This is unfortunate, as they may provide vital information regarding serotonin receptor subtypes that are involved in specific human cognitive domains. Finally, the prolonged use of MDMA (3,4-methylenedioxymethamphetamine; ecstasy) is according to some authors associated with quite selective neurotoxic damage to serotonin neurones in animal experiments (McCann et al., 2000). Hence, assessment of the cognitive changes in frequent MDMA users may be used to provide indirect evidence on the role of serotonin in human cognition. Although acute MDMA intake also invokes potent stimulatory effects on serotonin neurotransmission, additional pharmacodynamic properties of MDMA, most notably those pertaining to the dopamine (DA) system (Green et al., 2003), renders MDMA less suitable for examination of specific serotonin mechanisms.

The evidence for a role of serotonin in human cognition will be discussed in two sections, one dealing with healthy volunteer studies, the other with studies in selected (clinical) populations. The distinction is based on the premise that the cognitive effects of various serotonin manipulations may vary according to baseline central serotonin activity. In several conditions, e.g. depression, and possibly also Alzheimer's disease and normal aging, both serotonin hypofunction and cognitive decline is observed. At this point, the causal relation between a compromised central serotonin system and the observed cognitive deficits in these and other conditions has not been clearly established, but there is increasing evidence that low serotonin is at least partially linked to the cognitive problems. Hence, these populations may specifically benefit from pro-serotonergic pharmacological therapies that shift central serotonin neurotransmission towards a more optimal level, thus restoring cognitive function. By contrast, in healthy volunteers, no performance deficits due to a compromised serotonin system are to be expected. As they already operate close to their optimal cognitive performance level, cognition enhancement is generally difficult to achieve, by any means. Possibly, stimulation of the serotonin system in this group may even result in diminished performance, if optimal serotonin 
modulation is disrupted. As such, assessing the cognitive sequelae of altered central serotonin levels in healthy volunteers is a valuable method to identify the fundamental role of serotonin in cognition, providing leads for investigating possible serotonergic mechanisms underlying impaired cognition in specific clinical populations, and ultimately providing the rationale for serotonergic treatment options.

\section{Serotonin and human cognition: evidence from healthy volunteers studies}

Recent findings from psychopharmacological studies with healthy volunteers have identified a number of cognitive domains that appear to be sensitive to changes in serotonin neurotransmission in the brain. The most consistent effects relate to longterm memory functions, certain attentional functions, notably focused and sustained attention, and specific executive functions that mediate behavioural adaptations to changing environmental demands: a function referred to as cognitive flexibility. In the following section, the evidence for serotonin involvement in these functions from experiments with healthy human volunteers is reviewed.

\section{Serotonin and human memory functions}

Memory is a multifaceted cognitive function relating to the acquisition, storage and preservation of information for short and longer time periods, and the subsequent retrieval of this information. Numerous memory tests are available, varying in terms of the types of information, temporal characteristics and specific processes that are targeted. List learning paradigms are often used to test various aspects of declarative memory (memory for facts). Such tests typically consist of one or more acquisition trials in which information is presented, followed by recall and recognition trials to assess retrieval and storage, respectively. Varying the time interval between presentation and assessment allows for a differentiation between short- and longterm memory functioning.

The serotonergic system appears to be involved in specific human memory processes. The perhaps most compelling evidence for this was obtained in a number of experiments in which ATD was used to lower central serotonergic activity in healthy volunteers. These studies demonstrated that lowering of serotonergic activity is associated with a quite specific impairment of long-term memory performance (Riedel, Klaassen, \& Schmitt, 2002). In one of the initial experiments, Riedel et al (1999) showed that ATD reduced the ability to actively recall, as well as recognize, 
words from a previously presented word list. The effect was only apparent after a 30 minutes delay between presentation and assessment, but not when word recall was assessed immediately after presentation. This pattern lead the investigators to the conclusion that consolidation of new information into long-term memory was compromised by a reduction of central serotonin activity, but that short-term memory functions appear to be spared. Several studies have subsequently confirmed the notion that serotonin is specifically involved in long-term memory functioning. ATD was found to specifically reduce delayed recall and/or delayed recognition of visually presented words (Harrison et al., 2004; Kilkens et al., 2004; Rowley et al., 1998; Schmitt et al., 2000; Sobczak et al., 2002), spoken words (McAllister-Williams et al., 2002), pictures (Sobczak et al., 2002) and even abstract figures (Rubinsztein et al., 2001), whereas two studies failed to detect ATD-induced changes in memory for words (Hughes, Gallagher et al., 2003; Shansis et al., 2000). The authors of the latter study suggest that the results may have been hampered by modest levels of tryptophan depletion. Overall, results seem to indicate that ATD-induced memory impairment occurs independent of the nature of the stimuli and suggest that serotonin modulates long-term memory processes at a fundamental level. The specificity of this effect for the depletion of tryptophan was demonstrated in two studies where the memory consolidation deficit was found in the ATD condition but not when other amino acids, lysine (Klaassen et al., 1999) and tyrosine/ phenylalanine (Harrison et al., 2004) respectively, were depleted within the same group of volunteers. Furthermore, it was demonstrated that ATD does not affect long-term memory retrieval or recognition when the depletion is induced after learning and consolidation of a word list (Schmitt et al., 2000). Event related potential (ERP) recordings further showed that impaired long-term memory recall for words after ATD occurred without changes in the magnitude or topography of the neural correlates of retrieval functions (McAllister-Williams et al., 2002). These results suggest that impaired storage and/or consolidation of new information, rather than the ability to access and retrieve information, underlies poor long-term memory performance after serotonin depletion.

The results emerging from ATD studies indicate that reduced long-term memory performance is not likely to be attributable to consistent changes in perceptual functions, information processing, attention or executive functions (Gallagher et al., 2003; Harrison et al., 2004; Park et al., 1994; Riedel, Klaassen et al., 1999; Schmitt et al., 2000). In fact, there is some indication that some attentional 
functions, i.e. selective attention (see below), and memory related executive functions, i.e. strategy driven retrieval from semantic memory (word fluency), may benefit from a reduction of serotonin neurotransmission (Schmitt et al., 2000). Also, the absence of evident changes in self-reported mood ratings of healthy volunteers following ATD (Booij et al., 2003; Riedel, Klaassen, \& Schmitt, 2002; Young \& Leyton, 2002) suggests that diminished memory function is not mediated by mood changes. A detailed analysis of the long-term memory impairment following ATD, however, revealed that recall of words with a positive and neutral affective valence was specifically impaired, whereas recall of negative words was unaffected (Klaassen et al., 2002). The authors conclude that serotonin depletion shifts affective memory bias towards words with a negative affective valence. Supporting this notion, recent data show that ATD was associated with a specific decrement in memory for positively loaded words (Kilkens et al., 2004). The results are indicative of a mood congruent memory bias caused by a lowering of mood and suggest an interaction between subtle mood effects and memory effects induced by low serotonin activity.

In contrast with the quite consistent findings on serotonin depletion and memory impairment, the studies on the effects of serotonin stimulation on memory functions in healthy volunteers are scarce and have yielded rather inconsistent results. No changes in long or short-term memory performance were seen after $50 \mathrm{mg}$ acute (Coffey et al., 1994) or 50-100 mg subchronic (Schmitt et al., 2001; Siepmann et al., 2003) administration of the SSRI sertraline in healthy volunteers. Citalopram administration has been associated with decreased long-term memory performance in healthy middle-aged subjects. After 14 days of treatment with citalopram: $20 \mathrm{mg}$ on days 1-7; $40 \mathrm{mg}$ on days 8-14), subjects reproduced 1.5 words less (5\%) at delayed recall (Riedel et al., 2005) Impairment of delayed word recall was also found after sub-chronic administration of 20-40 $\mathrm{mg}$ paroxetine in healthy middle-aged subjects (Schmitt et al., 2001). Memory function, particularly long-term memory retrieval and storage, was impaired following intravenous administration of $7 \mathrm{~g}$ TRP (Sobczak et al., 2003). However, the relatively high dose of TRP had marked sedative effects, probably due to the conversion of TRP to melatonin, as was apparent from subjective rating scales and spontaneous reported side effects. Moreover, sedation was positively correlated with memory performance, suggesting that the observed memory effect was primarily mediated by melatonin accumulation and possibly, to some extent, by sedative effects of serotonin stimulation itself. Finally, memoryenhancing effects of SSRIs have also been reported. An acute intravenous 
administration of $10 \mathrm{mg}$ of the selective serotonin reuptake inhibitor (SSRI) citalopram in healthy volunteers was shown to facilitate verbal memory consolidation (Harmer et al., 2002). After subchronic administration of the SSRI sertraline in healthy elderly subjects, sertraline plasma levels were correlated positively with short-term memory scores on day 7 (low dose: $50 \mathrm{mg}$ ) and long-term memory scores on day 21 (high dose: $150 \mathrm{mg}$ ), while no correlation with memory was found on day 14 (intermediate dose: $100 \mathrm{mg}$ ) (Furlan et al., 2001). A study of the acute effects of $10 \mathrm{mg}$ ipsapirone, a $5-\mathrm{HT}_{1 \mathrm{~A}}$ agonist, and $0.5 \mathrm{mg} / \mathrm{kg} \mathrm{m}-\mathrm{CPP}$ (metachlorophenylpiperazine), a 5- $\mathrm{HT}_{2 \mathrm{C}}$ agonist, showed that ipsapirone impaired immediate recall in healthy volunteers (Riedel, Klaassen, Griez et al., 2002).

Data from serotonin depletion studies provide ample evidence for a role of the central serotonergic system in long-term memory functioning. It seems that increasing serotonin activity in healthy volunteers, however, does not necessarily lead to memory improvement. In fact, some findings suggest that overstimulation of the serotonin system actually induces memory impairment. The absence of consistent beneficial effects of serotonin stimulation may indicate that in healthy volunteers, under normal conditions, the level of central serotonin activity is not a limiting factor for optimal memory functioning. From the current data, serotonin manipulations do not seem a particular viable method to enhance memory in healthy subjects, although reports of improved memory following serotonin stimulation certainly merit further investigation.

\section{Serotonin and human attention functions}

\section{Focused attention}

Focused attention refers to the ability to attend a relevant stimulus or stimulus attribute while ignoring irrelevant information. In focussed attention tasks a predefined informational cue, indicating the required response, is accompanied by distracting information, which causes interference. A classic and often used test of focused attention is the Stroop Colour Word Test (Stroop, 1935), in which subjects have to ignore the content of a series of printed colour names and name the incongruent ink colours (e.g. "yellow" printed in blue ink). Interference can also be introduced in flanker task (Eriksen \& Eriksen, 1974) by simultaneous presentation of distracting information in proximity of the target stimulus, e.g. a central arrow that indicates the required response is flanked by arrows that point in the opposite direction. Another example of a focused attention task is the shadowing condition of 
the dichotic listening task (Kimura \& D'Amico, 1989), in which series of numbers spoken in one ear have to be processed while ignoring simultaneously spoken numbers in the other ear.

Data from several ATD experiments indicate a putative role of serotonin in the modulation of focused attention. In a healthy volunteer study, ATD was found to reduce interference in a Stroop Colour Word Test and increase performance on the focussed attention component of the dichotic listening task (Schmitt et al., 2000). These findings are in line with previous ATD studies reporting decreased interference in the Stroop test (Rowley et al., 1998) and speeded responses to incompatible stimuli (e.g. the word "left" presented at the right side of the screen) after ATD (Coull et al., 1995). The positive effects of ATD on Stroop and dichotic listening performance could not be replicated in a subsequent study, although the data tended to show a similar pattern(Sobczak et al., 2002). The authors argued that the heterogeneity of their study population, in terms of age and psychiatric family loading, may have lead to reduced sensitivity. Lack of ATD effects on Stroop performance have also been reported in two other studies (Danjou et al., 1990; Gallagher et al., 2003). A recent finding, however, of a dose-related increase in performance on the Stroop test in a study comparing the effects of two levels of ATD in depressed patients provides strong evidence for serotonin involvement in focused attention (Booij et al., 2005). The role of serotonin in focused attention is further substantiated by EEG (electroencephalography) and MEG (magnetoencephalography) measures in healthy volunteers after ATD. Both EEG and MEG correlates of auditory attention (mismatch negativity, N2b and N2 wave) suggested that ATD decreased involuntary attention shifting to task-irrelevant sound changes and thus modulated resource allocation to the task-relevant activity (Ahveninen et al., 2002).

In healthy volunteers, there is no clear evidence that serotonin stimulation influences measures of focused attention, although it must be noted that only a few studies have addressed this issue. Performance on the Stroop test was unaffected by 14-15 days of administration of 20-40 mg paroxetine (Hindmarch \& Harrison, 1988; Kerr et al., 1992; Schmitt, Ramaekers et al., 2002) and 50-100 mg sertraline (Schmitt, Ramaekers et al., 2002). No changes in performance on a dichotic listening task, in an identical version that was shown to be sensitive to ATD, were found following subchronic administration of 50-100 mg sertraline and 20-40 mg paroxetine (Schmitt, Ramaekers et al., 2002). A 7g intravenous TRP challenge to stimulate serotonin neurotransmission resulted in diminished speed of responding to incompatible 
stimuli in a left-right task TRP (Sobczak et al., 2003), in line with the effects of ATD on focused attention. However, the effect was only observed in those subjects with a first degree relative with bipolar disorder. Furthermore, the effect did not extend to other tests of focused attention in the study, i.e. the Stroop test and dichotic listening.

\section{Sustained attention (vigilance)}

Sustained attention is the ability to direct and focus cognitive activity or alertness on specific stimuli over a prolonged period of time. In tests of sustained attention or vigilance stimuli are usually intermittent, unpredictable and infrequent. The prototypical vigilance task is the Mackworth Clock task (Mackworth, 1950). This task was developed during World War II to simulate radar monitoring operations. A computer screen displays a circular arrangement of dots simulating the second marks on a clock. Dots are briefly illuminated in clockwise rotation. At irregular intervals a "double jump" occurs by skipping one of the dots in the normal sequence. These are the signals that an observer has to detect and respond to. It has been shown that vigilance performance declines steeply as a function of time on task (Mackworth, 1950).

Augmentation of serotonin neurotransmission has been shown to impair human vigilance performance, i.e. the ability to remain alert for prolonged periods of time. Ramaekers et al. compared the acute and subchronic effects of fluoxetine $20 \mathrm{mg}$ on sustained attention to those of placebo in a double-blind, cross-over study involving 18 healthy volunteers. Sustained attention was assessed using the Mackworth Clock paradigm (Mackworth, 1950) on days 1, 8 and 22 of treatment. A reduction in sustained attention was observed throughout the fluoxetine treatment period. The selective impairing effects on vigilance and sustained attention have also been observed following two weeks of administration of incremental dose regimens of venlafaxine $(75-150 \mathrm{mg})$ to healthy volunteers. Little effect of venlafaxine on sustained attention was seen on the first day of treatment but the effect increased to become significant after one week of treatment. Subjects seemed to notice venlafaxine's effect on vigilance as they subjectively rated their alertness less as compared to placebo. Single and repeated doses of paroxetine $30 \mathrm{mg}$ did not affect vigilance performance as compared to placebo in healthy volunteers (Deijen et al., 1989). However in this study maprotiline $100 \mathrm{mg}$ failed to affect vigilance performance also, even though this drug is known to produce drowsiness. Deyen et al (Deijen et al., 1989) did not reveal any task parameters relevant to an operational 
Mackworth clock paradigm but their vigilance data suggests that their version may not have been sensitive enough to assess drug-induced changes. Schmitt et al (2002) on the other hand did report a paroxetine induced vigilance decrement. They compared the effects of subchronic treatment with sertraline $(50-100 \mathrm{mg})$ and paroxetine $(20-40 \mathrm{mg})$ on sustained attention in a placebo controlled study in 21 healthy volunteers. Paroxetine significantly reduced performance on the Mackworth Clock task, i.e. it decreased the number of correct detections and increased mean reaction time for correct detections. In contrast, sertraline did not reduce the number of correct detections and decreased mean reaction time. Similar results were reported in a placebo controlled study by Riedel et al (2005). They found that citalopram impaired vigilance performance in healthy volunteers during the first week of treatment with $20 \mathrm{mg}$ and during the second week of treatment with $40 \mathrm{mg}$ daily. In contrast, no vigilance impairment was found during sertraline $(50-100 \mathrm{mg})$ treatment. The magnitude of citalopram induced impairment in the Mackworth Clock task was comparable to that previously observed for fluoxetine, venlafaxine and paroxetine (O'Hanlon et al., 1998; Ramaekers et al., 1995; Schmitt, Ramaekers et al., 2002). Edgar et al (Edgar et al., 2004) assessed vigilance performance in 2 groups of healthy volunteers who received 16 days of treatment with escitalopram or sertraline. The Mackworth Clock Test was administered at baseline and on day 16 at 2 and 4 hrs post dosing. Relative to baseline escitalopram decreased vigilance accuracy, but only at 2 hrs post dosing. Harmer et al (2002) failed to measure any affect of citalopram on sustained attention while using another vigilance paradigm: i.e. Rapid Digit Information Processing. It is unclear however whether this paradigm measures the same aspects of vigilance as the Mackworth Clock Test.

Thus, sertraline is the only SSRI studied so far with no detrimental effects on vigilance. It has been suggested that the latter may be due to the fact that sertraline also possesses an ancillary mechanism that facilitates cortical arousal; i.e. blockade of DA reuptake. In such case vigilance impairment following serotonin stimulation may be counteracted by the vigilance promoting effects of DA stimulation (Schmitt, Ramaekers et al., 2002).

Studies employing the Mackworth Clock paradigm thus all seem to point out that enhancing serotonin in normal individuals consistently impairs vigilance. The mechanism underlying this effect however has not been fully elucidated. A reduction in noradrenergic (NA) activity does not seem to play a major role as simultaneous enhancement of NA with serotonin by venlafaxine was previously shown not to 
counteract the vigilance decrement (O'Hanlon et al., 1998). Similarly, decreased catecholamine availability after tyrosine/phenylalaline depletion did not affect performance of healthy volunteers in a digit-vigilance task (Harrison et al., 2004). Animal studies have also indicated that NA is not specifically associated with the maintenance of vigilance (Delagrange et al., 1993; McGaughy et al., 1997), but is more likely to control phasic arousal and hence may be related only when a sensory pathway habituation type of vigilance decrement is at stake. DA on the other hand has been associated with tonic arousal and readiness to respond (Pribram \& McGuinness, 1975; Robbins, 1997). Stimulation of DA transmission by amphetamine and methylphenidate has been shown to improve vigilance performance (Koelega, 1993). Vigilance impairment produced by SSRIs may involve both serotonin and DA neural circuits. It is interesting in this context that simultaneous depletion of serotonin and catecholamines did impair sustained attention of healthy volunteers in a digit-vigilance task (Hughes, Matrenza et al., 2003), whereas selective depletion of serotonin and catecholamines did not (Harrison et al., 2004). It has been shown that serotonin projections from the raphe nucleus exert a tonic inhibitory influence over the mesocortical, nigrostriatal and mesolimbic DA systems. Thus augmentation of serotonin can be predicted to further inhibit DA transmission in DA pathways (Schmitt, Ramaekers et al., 2002).

Several researchers have claimed that SSRIs can increase general CNS (Central Nervous System) arousal and attention (Kerr et al., 1991). This claim was generally based on the notion that SSRIs are capable of increasing the critical flicker fusion (CFF) threshold in healthy volunteers. CFF is a task that requires subjects to discriminate flicker from fusion in a set of light emitting diodes held in foveal fixation. The psychological functions that are being measured with CFF however have never been truly established. In general, drug induced decrements in CFF threshold are believed to reflect sedative aspects from a particular compound, whereas elevation of CFF threshold might indicate the opposite: i.e. activation. If true, data demonstrating elevated CFF thresholds after SSRIs administration could be taken to support the notion that these drugs improve (sustained) attention. However it has also been argued that a rise in CFF threshold may also result from mydriasis produced by SSRIs rather than a change in arousal (Ramaekers et al., 1995). Pupil diameter is an important determinant of CFF threshold and several studies have shown that serotonergic drugs may alter pupil size. More in particular it has been shown that SSRIs may cause a $2 \mathrm{~mm}$ increase in pupil size after single and repeated 
doses (Deijen et al., 1989; McGuirk \& Silverstone, 1990; Raptopoulos et al., 1989; Saletu \& Grunberger, 1988; Schmitt, Riedel et al., 2002). It is noteworthy in this respect that all of the studies reporting SSRI induced elevation of CFF threshold failed to control pupil size as a possible confounder, and that investigators that did control pupil diameter while measuring the effects of SSRIs on CFF did not report any significant elevations in CFF threshold (Ramaekers et al., 1995 ; Schmitt, Riedel et al., 2002). Only Mattilla et al (Mattila et al., 1988) reported a slight CFF threshold elevation from baseline following the administration of an SSRI (sertraline 50mg) in an CFF paradigm controlling for pupil size. However, the authors believed that this finding was invalid as it was confounded by irregular baseline deviations. When compared directly to placebo, no significant change in CFF was reported following administration of sertraline. Thus, studies showing SSRI induced elevations in CFF threshold do not necessarily contradict with studies showing that SSRIs decrease vigilance or sustained attention. CFF data should always be interpreted with caution, particularly when confounding factors such pupil size are uncontrolled for.

\section{Serotonin and cognitive flexibility}

Cognitive flexibility refers to the ability to adapt ongoing behaviour to changes in reinforcement. It is a multi component process, involving several cognitive subprocesses such as feedback processing, behavioural adaptation, learning of new stimulus-reward associations and inhibition of the previously stimulus-reward associations (response inhibition). Tasks measuring cognitive flexibility include reversal learning tasks, decision making tasks or gambling tasks, the intra dimensional/ extra dimensional shift (ID/ED) task, and the Wisconsin Card Sorting Task (WCST). Typically, these tasks require the subjects to choose the appropriate response, and adjust their response strategy based on the feedback or cues they receive (correct/incorrect, reward/punishment, probabilities). Especially the orbitofrontal and cingulate cortices have been associated with cognitive flexibility (Catafau et al., 1998; Cools et al., 2002; Krawczyk, 2002; Kringelbach \& Rolls, 2003; Rezai et al., 1993; Rogers, Owen et al., 1999; Volz et al., 1997). Neuroimaging studies have implicated these brain regions in various cognitive processes that are important for flexible behaviour, including cognitive processing of affective meaning and reward, and inhibitory control (see (Murphy et al., 2003; Rogers, Owen et al., 1999; Rogers et al., 2003)). 
In a typical reversal learning task the subject has to choose between two stimuli that are presented simultaneously. The assignment is to identify the correct or most profitable stimulus based on the feedback that is given after each response. Once the correct association is learned, the reinforcement rules are reversed the subject has to adjust its responding to the new stimulus reward association (reversal shift). Several animal studies have implicated serotonin in reversal learning (see (Clark et al., 2004; Clarke et al., 2004). In healthy human volunteers, ATD impaired the ability to perform a reversal shift in an ID/ED task, as was apparent from an increase in erroneous responses after a reversal shift (Park et al., 1994; Rogers, Blackhaw et al., 1999). Impaired performance after ATD was also observed in a probabilistic reversal learning task, although deficits were observed for speed, rather than accuracy (Murphy et al., 2002). In a recent fMRI study (Evers, Cools et al., 2005), ATD did not affect behavioural indices of reversal learning, increased activation in the dorsomedial PFC after ATD was found when subject adapted their behaviour after a reversal. There was a trend that the increased activation in the dorsomedial PFC extended to all the events where negative feedback was received. It was suggested that decreased serotonin may impair cognitive flexibility by increasing the sensitivity for negative feedback. Lack of effects of ATD on reversal learning, as measured by an ID/ED task (Hughes, Gallagher et al., 2003), WCST (Gallagher et al., 2003; Hughes, Gallagher et al., 2003) and a probabilistic reversal learning task involving multiple reversal shifts (Evers, Tillie et al., 2005), have also been reported. While the apparent inconsistencies may be attributed to methodological issues, i.e. small sample size (Murphy et al., 2002), timing of the post-treatment assessments (Evers, Tillie et al., 2005) and modest levels of tryptophan depletion (Hughes, Gallagher et al., 2003), it is clear that further research is required to confirm and specify the role of serotonin in reversal learning.

The ability to make decisions that are most favourable in term of reward, and to adjust choices to varying conditions of potential reward and punishment, has also been associated with serotonin. Decision making tasks assess the quality and speed of choices that a subject makes under various conditions of potential gains or losses and associated chances of winning or losing. ATD tended to increase deliberation times, i.e. reduced the speed of decision making, particularly when probabilities of making the correct choice were higher. Furthermore, quality of decision making, i.e. choosing the most likely of the two response options, was reduced. ATD did not affect risktaking, i.e. the size of the bets that subjects placed on a particular response, or 
impulsivity in the selection of the bets (Rogers, Everitt et al., 1999). In subsequent study (Rogers et al., 2003) it was shown altered decision making after ATD may be mediated by changes in reward processing. More specifically, ATD was found to attenuate the subjects' ability to discriminate between different magnitudes of expected gains. Processing of punishment cues (discrimination between small and large losses) or probability cues (discrimination between low and high probability of winning) was not affected by ATD. The authors propose that complex interactions between serotonin and mesolimbic DA system may underlie the observed changes in affective processing that is associated with the orbitofrontal cortex. In contrast, Anderson et al (Anderson et al., 2003) failed to detect any ATD-induced changes in decision making in a gambling task in which probabilities of winning and potential gain were manipulated. As was also noted by the authors, the parallel group design and modest sample sizes (15 ATD versus 13 placebo subjects) probably limited the study's statistical power.

Currently, there are no reports on the effects of serotonin stimulation on cognitive flexibility in healthy volunteers.

\section{Clinical aspects}

\section{Depression, serotonin and cognition}

Impaired cognitive function is one of the key features of a depressive disorder. The cognitive deficits that are associated with a depressive episode were initially thought to be secondary symptoms, mediated by lowered mood, abnormal sleep, loss of interest or motivational deficits. These factors may certainly contribute to abnormal cognitive functioning in depression, however, it has now become clear that cognitive impairment is one of the core symptoms of depression and may occur independent of mood changes.

A depressive episode is often accompanied by extensive cognitive impairment across a wide range of cognitive domains. Impairment of long-term memory functioning, particularly impairment of encoding and retrieval, is frequently and quite consistently observed in depression (Austin et al., 2001; Brand et al., 1992; Burt et al., 1995; Den Hartog et al., 2003; Zakzanis et al., 1998). In addition, attention deficits (Landro et al., 2001; Purcell et al., 1997) and impairment of executive functions, for example difficulties on measures of verbal fluency, attentional setshifting and cognitive flexibility, are reported (Austin et al., 2001; Austin et al., 1992; Elliott, Baker et al., 1997; Elliott, Sahakian, Herrod et al., 1997; Elliott et al., 1996; 
Elliott, Sahakian, Michael et al., 1997; Fossati et al., 2002; Murphy et al., 2001; Murphy et al., 2003; Stordal et al., 2004). Given that central serotonergic dysfunction is considered to be one of the neuronal substrates of depression(Maes \& Meltzer, 1995), one could speculate at least part of the observed cognitive deficits are directly attributable to diminished brain serotonin neurotransmission. It is particularly noteworthy that the observed deficiencies in memory encoding and/or consolidation during depression are highly comparable to the memory effects that are seen following ATD in healthy volunteers (Riedel, Klaassen, \& Schmitt, 2002). Similarly, reduced cognitive flexibility in depression seems to be in line with ATD-induced impairment of cognitive flexibility in healthy volunteers. It must be noted, however, that depression is also associated with deficits in central catecholaminergic (i.e. NA and DA) activity (Anand \& Charney, 2000; Ordway et al., 2002) neuroendocrine abnormalities, (e.g. cortisol hypersecretion) (Porter et al., 2004; Schatzberg et al., 2002) and immunological changes(Kronfol, 2002; O'Brien et al., 2004). It is therefore likely that the pattern of depressive symptoms, including the cognitive changes, is the result of a complex interplay between various neurobiological, neuroendocrine and immunological factors.

Many antidepressant drugs stimulate serotonergic neurotransmission by either blocking the reuptake of serotonin into the presynaptic neuron or inhibiting the enzyme responsible for the degradation of serotonin (monoamine oxidase). The most obvious and straightforward approach to gain insight in the role of serotonin in cognitive deficits in depression is to evaluate the cognitive effects of treatment of depressed patients with pro-serotonergic antidepressants. Successful antidepressant treatment alleviates depressive symptoms and is generally associated with improvement of cognitive performance. Clinical improvement and enhanced memory performance was observed after six weeks of treatment with fluoxetine in depressed patients (Richardson et al., 1994). In elderly depressed patients, improvement of short- and long-term memory functions, visuospatial capabilities, arousal measures, and psychomotor speed was observed after 6 weeks administration of fluoxetine (Fairweather et al., 1993; La Pia et al., 2001). Similarly, in elderly depressed patients, twelve weeks of treatment with sertraline reduced depression symptoms and improved short- and long-term memory function (Bondareff et al., 2000; Finkel, Richter, \& Clary, 1999). Also in elderly depressed patients, 12 weeks treatment with fluoxetine or sertraline improved clinical ratings, short- and long-term memory function and performance on the digit symbol 
substitution test (Doraiswamy et al., 2003; Finkel, Richter, Clary et al., 1999; Newhouse et al., 2000). It is worth mentioning that in those studies in which the effects of non-selective antidepressants were also assessed, notably mianserin (La Pia et al., 2001), nortriptyline (Bondareff et al., 2000; Doraiswamy et al., 2003; Finkel, Richter, \& Clary, 1999), imipramine (Koetsier et al., 2002) or amitriptyline (Richardson et al., 1994), these drugs generally had comparable efficacy in terms of the therapeutic effect, but produced significantly less cognitive improvement or in some cases cognitive deterioration. These findings highlight the unfavourable cognitive side effects produced by anti-cholinergic, anti-noradrenergic and antihistaminergic drug actions.

A major methodological caveat in the aforementioned clinical trials is the absence of a control condition. Cognitive performance is known to improve with repeated assessments due to practice effects, procedural learning and familiarity with the testing protocols, leading to reduced anxiety. The importance of a control group is illustrated by a study by Nebes et al. (Nebes et al., 2003). In this study, improvement of cognitive performance was observed following 12 weeks of treatment with paroxetine and nortriptyline in elderly depressed patients. However, a similar improvement of cognitive performance was observed in a healthy matched control group that underwent an identical study procedure (except for drug administration). The results could not be explained by treatment response, benzodiazepine comedication, age of depression onset, or by co-existence of early dementia. Consequently, it was concluded that there was also no evidence for nortriptyline or paroxetine having an effect on cognition in elderly depressed patients. Others did find that nortriptyline and paroxetine treatment improved cognitive performance in elderly depressed patients compared to a healthy control group, although the effect was modest and limited to those subjects that were modestly to severely cognitive impaired at baseline (Butters et al., 2000). Sertraline or desipramine treatment did not significantly alter cognitive performance (reaction time, DSST, trail making) in depressed elderly patients, when compared to placebo treatment. However, clinical efficacy also did not differ between treatment groups and placebo, according to the authors, due to modest sample sizes, mild to moderate severity of depression, and a high placebo response. In another study, depressed patients, when compared to a healthy control group, showed improved performance on a continuous performance task, along with clinical improvement, following 4 weeks of fluvoxamine administration (Koetsier et al., 2002). In a group of depressed inpatients receiving 
fluoxetine, performance on a series of tests assessing fine motor skills was markedly improved after 5 weeks of treatment, compared to healthy controls undergoing the same assessments (Sabbe et al., 1996). Overall, these findings suggest that cognition does tend to improve with successful antidepressant therapy, but without ample control for spontaneous changes in performance there is a risk of overestimating the actual cognitive effect of antidepressant treatment.

A second important issue is whether or not cognitive improvement after serotonergic antidepressant treatment is a direct effect of enhanced serotonin neurotransmission or the secondary result of alleviation of the depressive illness in general, e.g. removal of mood, motivational and sleep abnormalities. Mixed results were obtained when clinical improvement was correlated with improvement of overall cognitive performance (combined changes in memory and DSST scores) following 3 months of nortriptyline, sertraline, or fluoxetine treatment. While significant correlations were found for patients treated with sertraline and, albeit weaker, for nortriptyline, no correlation was found in patients treated with fluoxetine. Thus, although cognitive improvement may be linked to clinical efficacy, this does not seem to be a prerequisite. The authors suggested that both a direct pharmacological effect, i.e. serotonin stimulation, as well as improvement of depression may affect cognition in depressed patients (Doraiswamy et al., 2003). Also, no correlation between improvement of clinical depression scales and improved performance on a continuous performance test could be found following 4 weeks of imipramine or fluvoxamine treatment (Koetsier et al., 2002). There is some evidence that serotonin stimulation may indeed improve cognition directly, independent of relieve of other depressive symptoms. After 6 weeks of trazodone treatment, clinical remission and improved verbal long-term memory function (in spite of trazodone's sedating effects) was observed in a group of middle-aged depressed patients. In the ensuing placebo washout period of one week, memory performance reverted to baseline levels while depression ratings remained below depression threshold. Thus, memory function appeared to fluctuate mainly as a function of serotonin activity, rather than depressive symptomatology (Riedel, Schoenmakers et al., 1999). In another study, the noradrenergic antidepressant desipramine and the SSRI fluoxetine were found to be equally effective in terms of their clinical effects. Improvement of memory, however, was only apparent with the serotonergic antidepressant fluoxetine. This does not only suggests a dissociation between clinical response and memory improvement, but is also in keeping with the specific role of serotonin in 
memory processes (Levkovitz et al., 2002). One study has investigated the acute effects of $10 \mathrm{mg}$ ipsapirone, a $5-\mathrm{HT}_{1 \mathrm{~A}}$ agonist, and $0.5 \mathrm{mg} / \mathrm{kg} \mathrm{m}-\mathrm{CPP}$ (metachlorophenylpiperazine), a 5- $\mathrm{HT}_{2 \mathrm{C}}$ agonist, on cognition and mood in medication-free, young and middle-aged, depressed patients and healthy controls (Riedel, Klaassen, Griez et al., 2002). Ipsapirone tended to improve short-term memory in patients, but impaired short-term memory in controls, whereas neither group showed any ipsapirone-induced changes in mood. M-CPP, on the other hand, impaired performance on reaction time tests in all subjects, and visual search efficiency in patients only. After $\mathrm{m}-\mathrm{CPP}$, depression and tenseness ratings were increased in patients, and increased fatigue was seen in all subjects. The pattern of results seem to indicate that certain transient mood changes may affect certain aspects of performance: increased fatigue was accompanied by increased reaction times, and depression and tenseness were associated with visual search deficiencies. Altered memory functioning, however, appeared to occur without any overt changes in mood states. Moreover, while $5-\mathrm{HT}_{1 \mathrm{~A}}$ agonism impaired memory in healthy subjects, facilitating effects were found in patients. The authors speculate that the latter finding may be related to hippocampal 5- $\mathrm{HT}_{1 \mathrm{~A}}$ desensitation in patients (Riedel, Klaassen, Griez et al., 2002). As such, it may be tentatively hypothesized that the differential memory effects may reflect normalization versus overstimulation of hippocampal 5$\mathrm{HT}_{1 \mathrm{~A}}$-mediated serotonergic neurotransmission in patients and healthy subjects, respectively.

\section{Serotonin in neurodegeneration: ageing and dementia}

Improved understanding of the nature of alterations in the serotonin system occurring in aging, late-life depression, and dementia has important implications for testing hypotheses of neurochemical mechanisms underlying the aging process and age-related neuropsychiatric disease. Cognitive performance enhancing effects of serotonin manipulations could be useful in various clinical populations in whom serotonin function is thought to be compromised. Given the specific involvement of serotonin in memory consolidation, neurodegenerative diseases may involve the serotonergic system. To consider are ageing, Alzheimer's Disease (AD) and frontotemporal dementia. The working hypothesis is that therapies that stimulate the serotonergic system in these populations might be beneficial.

There is increasing evidence for alterations in the function of the serotonin system in $\mathrm{AD}$, which may be responsible for many of the behavioural aspects of the 
disease including the frequent coexistence of depression. The literature implicating serotonin dysfunction in depression, aging and AD is based largely on the results of indirect measures, including animal models, post mortem human studies, and peripheral measurements of serotonin binding (Meltzer et al., 1998). In aging, it has recently been shown that $5-\mathrm{HT}_{2 \mathrm{~A}}$ receptor binding decreases dramatically in a variety of brain regions up through midlife (Sheline et al., 2001). In AD, serotonin dysregulation is reported separately or in conjunction with that of the cholinergic system, due to interactions at the receptor level, in particular in septo-hippocampal cholinergic neurones as well as in cholinergic neurones from the nucleus basalis to the cortex and the amygdala (Buhot et al., 2000). In a study by Porter (Porter et al., 2003) AD patients were administered ATD and their cognitive responses were compared to those of age-matched controls. No specific differences were found with respect to cognitive responses to ATD between the AD and control groups. However, the observed effects of ATD both in the normal aged and in the AD groups seemed to differ from those seen in healthy young volunteers. An impairment of performance on the backward digit span was observed after ATD in both groups. Such impairment may well underlie other memory deficits induced by ATD, such as the observed ATD-induced impairment in the Paired Associates Learning test (Porter et al., 2003). However, no ATD-induced impairments were found on the Rey auditory verbal learning task and neither on the Rey Visual Design Learning Test. Ultimately, this could mean that memory consolidation impairment, acts as a marker of cognitive aging and although it is a sensitive indicator of ATD, might not be a relevant state marker of serotonin in AD. Previously, the same group (Porter et al., 2000) reported on the basis of differences between AD patients and healthy controls in their response to ATD, that using the 3MSE, an extended version of the Mini Mental State Examination scored on a scale of 1-100, AD patients responded to ATD by an impairment of performance on that scale whereas elderly controls did not. Porter et al. (2003) noted that this may reflect a ceiling effect on this scale in the elderly control group. We therefore remain with the fact that this was hitherto the first and only study to investigate the specific role of serotonin in aging and AD.

There are almost no reports of attempts to use SSRIs in AD and ageing specifically aimed at enhancing cognition. Partly, this is due to the fact that studies of SSRIs in ageing subjects are usually focused on alleviating low mood in late-life depression. In AD, studies of SSRIs have been reported to improve the non-cognitive symptoms rather than cognitive performance (Gottfries, 2001). Furthermore, a trial 
has been reported in which treatment of AD patients with cholinesterase inhibitors was compared to the same in addition with the SSRI citalopram. The outcome focused on attributing additional mood and behavioural symptoms improvement in the cholinesterase inhibitor + SSRI group (Moretti et al., 2002).

Rahman et al. (Rahman et al., 1999) have recently suggested that some of the symptoms associated with the frontal variant of frontotemporal dementia (fvFTD) are related to serotonergic dysfunction. This concerns marked deficits on tests sensitive to ventromedial prefrontal or orbitofrontal function (i.e. risk-taking, reversal learning), in the relative absence of impairments on tests sensitive to dorsolateral prefrontal function (i.e. spatial working memory and planning) (Rahman et al., 1999). Many of the symptoms of fvFTD are indeed currently treated using serotonin-boosting compounds, which are presumably aimed at the aforementioned brain regions and their associated behaviours such as impulsiveness, depression, alterations in eating habits and obsessions and compulsions (Rahman et al., 1999). Swartz et al. (Swartz et al., 1997) have demonstrated in preliminary studies that it is possible to ameliorate many of these specific symptoms, including impulsivity, depression, carbohydrate craving and compulsions, in fvFTD patients by using serotonin-boosting compounds (Swartz et al., 1997). Recently, however, administration of the SSRI paroxetine was found to impair the ability to perform reversal shifts in the ID/ED shift task in patients with frontotemporal dementia (Deakin JB, 2004).

\section{Acquired neurodegeneration: serotonergic vulnerability associated with MDMA-use}

Other evidence for cognitive dysfunction associated with putative serotonergic neurodegeneration may come from studies in former users of MDMA. Longlasting degeneration of long serotonergic fibres is seen after MDMA-use in animal studies (Ricaurte et al., 2000) and may also occur in human users (McCann et al., 2000; McCann et al., 1998; Reneman, 2001). These indirect observations of potential neurotoxicity are thought to underlie the overtly observed impairments of memory and mood in abstinent MDMA-users. More specifically it has been hypothesised that MDMA-users 'display cognitive deficits in tasks predominantly sensitive to temporal lobe dysfunction,' but no deficits in most tasks sensitive to prefrontal functioning (Fox et al., 2002). This dissociation might be related to a predominant physiological role of the serotonin system for mnemonic processes, as has been suggested by neuroanatomy (Buhot et al., 2000) and by ATD studies (Riedel, Klaassen, \& Schmitt, 2002). However, it may also be related to a particular vulnerability of the 
hippocampal complex to neurotoxic effects of MDMA (Gouzoulis-Mayfrank et al., 2003). Hence, ATD may be a model of the serotonergic depletion observed after MDMA use. A recent report comparing the metabolic and cognitive responses to ATD of former MDMA users showed that altered serotonin levels (as manifested by tryptophan levels) as well as impaired memory were the most sensitive markers of ATD's effects in ex-users of MDMA(Curran \& Verheyden, 2003). Furthermore, these measures were related in such a way that altered metabolism of tryptophan in exusers may reflect serotonergic vulnerabilty, either due to pre-morbid differences in their serotonin function or due to acquired drug-induced vulnerability (Curran \& Verheyden, 2003).

Treatment options for this group are predominantly antidepressants such as the reuptake inhibitors of serotonin, as they have been shown to not only elevate serotonin function, but have also been hypothesised to promote neurogenesis (Duman et al., 2001; Jacobs et al., 2000). Besides, estrogen, lithium and rolipram (phosphodiesterase inhibitor) have also been suggested (Duman et al., 2001).

\section{Cognitive dysfunction as a marker of low serotonin in diseases associated with peripheral} serotonergic dysfunction

A high proportion of cancer and hepatitis $C$ patients receiving immune-based therapy with the cytokine interferon-alpha, develop symptoms of depression and co-morbid cognitive symptoms such as memory impairment that are indistinguishable from those found in major depressive disorders (Capuron et al., 2002). The mechanism is poorly understood and could either lead to low serotonin via reduced appetite due to cytokine treatment or ATD by the cytokine-mediated induction of indoleamine 2,3dioxygenase (IDO), the enzyme that accelerates the catabolism of tryptophan, or both(Capuron et al., 2002). Antidepressant treatment could improve these patients' conditions of interleukin-induced depression and memory impairment, by preventing these symptoms to occur and improving compliance with interleukin therapy. The results of a recent placebo-controlled study of preventive SSRI treatment seemed to be largely consistent with this hypothesis (Capuron et al., 2003).

In a different group of cancer patients suffering from carcinoid tumors in the gastrointestinal tract which are known to probably cause a prolonged state of low serotonin by means of endogenous tryptophan depletion (Russo et al., 2003), a pattern of cognitive performance was observed that did not resemble that observed in depression, but rather consisted of improved measures of focused attention, similar 
to the effects of ATD previously described in healthy volunteers (Coull et al., 1995; Schmitt et al., 2000).

The sensory function of the intestine is highly serotonergically innervated (Camilleri, 2001). In patients with irritable bowel syndrome (IBS), serotonin may play a regulatory role in both gastrointestinal motility and sensitivity, as well as in affective dysregulation (Kilkens et al., 2003). In IBS there is also a hypothesis of endogenous tryptophan depletion and clinical benefits of several serotonergic agents have been shown (Lembo, 2000). Furthermore, it has been reported that greater brain serotonin synthesis in female IBS patients may be related to the pathological visceral pain processing of the IBS patients, a larger female predominance of the disorder, and the sex difference of the efficacy of the $5-\mathrm{HT}_{3}$ antagonist in treatment (Nakai et al., 2003). A recent attempt to show the association of peripheral and central serotonergic dysregulation, has shown that acute lowering of 5-HT synthesis enhances visceral urge and pain perception, while at the same time inducing memory impairment manifested as affective memory bias (i.e. preferential loss of memory for positive emotions), presumably through serotonergic modulation of the 'brain-gut axis' (Kilkens et al., 2004). Treatment of IBS with 5- $\mathrm{HT}_{3}$ antagonists is aimed at improving peripheral serotonergic function. $5-\mathrm{HT}_{3}$ antagonists have been studied for cognition enhancement, but clinical trials in $\mathrm{AD}$ and mild cognitive impairment have failed.

\section{Concluding remarks}

Healthy volunteer studies have provided support, of varying strength, for a role of serotonin in a number of human cognitive functions. The most robust evidence is available for human episodic memory functions and particularly the detrimental effects of low brain serotonin on long-term memory consolidation are quite consistently found. At the same time, it must be noted that the experimental data on serotonin inhibition and human memory are only available from studies using one specific method to lower central serotonin neurotransmission, namely ATD. The memory deficits that are associated with prolonged MDMA use, presumably caused by MDMA's neurotoxic effects on the serotonin system, provides indirect corroborating evidence, but currently there are no data on the memory effects of, for example, 5-HT antagonists. Furthermore, it appears to be difficult to ascertain any consistent effects of increased central serotonin on memory in healthy volunteers. A similar pattern is seen for the notion that serotonin is involved in focused attention and cognitive flexibility, which is largely based on results of ATD studies, and 
serotonin's effect on vigilance, which is solely based on SSRI studies. Overall, the findings of serotonin modulation of cognition in healthy volunteers are characterized by quite consistent and selective effects of cognitive changes, predominantly performance decrements, following serotonin manipulations in one direction (inhibition or stimulation), without clear evidence of mirrored behavioral effects of opposite serotonin manipulations. This may be due to several factors. As mentioned earlier, it may easier to induce performance decrements than to enhance cognitive performance in healthy volunteers who have a close to optimal performance level. Secondly, relatively few serotonin challenge studies have been performed, using varying pharmacological manipulations, dosages, methods of administration, and treatment regimes, which all can affect the actual level of central serotonin effects that are achieved, and hence the behavioral outcome of the manipulation. In addition, it is unclear if the relationship between serotonin activity and cognitive function is a linear one, or instead follows an inverted U-curve, as has been proposed for frontal DA activity and working memory functions (Lidow et al., 2003). In latter case, the behavioral effect would be the combined result of baseline serotonin activity and the level of pharmacological stimulation, with the possibility of performance decrements through overstimulation. At this point, however, there is insufficient data to either support or dismiss an inverted-U curve hypothesis.

While the potential for cognition enhancement by serotonin drugs in healthy volunteers remains to be established, restoration of impaired cognitive performance due to serotonin dysfunction is more viable potential target for serotonin drugs. The observed long-term memory deficits in depression are consistent with the ascribed role of serotonin in memory formation, and evidence suggest that serotonin stimulation may have particular beneficial effects on memory in these patients. Yet, a clear evaluation of the cognitive effects of 5-HT antidepressants in most clinical trials is hampered by a lack of control groups. Furthermore, 5-HT drug effects have been almost exclusively assessed in a limited subpopulation, namely elderly depressed patients. Also, from the existing data, it is difficult to dissociate the direct effects of serotonin augmentation on cognition from those that are mediated by alleviation of other symptoms of depression. Comparative studies, assessing the additional value, with regard to cognition, of 5-HT drugs over other effective antidepressant therapies, i.e. non-serotonergic antidepressants and non-pharmacological interventions, would provide further insight into this matter. Low serotonin has also been implicated in aging, AD and fvFTD, but it is unclear to what extent these serotonergic changes are 
responsible for the observed cognitive problems. Little work has been done in this area, but evaluation of the cognitive effects of pro-serotonergic drugs in these populations could lead to new fundamental insights in the neurobiology of these conditions, and, if successful, lead to new (co-) treatment strategies and drug development targets for these conditions.

Generally, impaired cognitive function is likely to be the resultant of a set of interacting neurobiological changes, rather than a single factor. Serotonergic dysfunction is unlikely to be the sole cause of cognitive impairment in any condition of illness, but given the mounting evidence of serotonin involvement in variety of cognitive functions, loss of integrity of the central serotonergic system may certainly contribute, in various degrees, to cognitive deterioration. As such, the serotonergic system has become a target for pharmacological cognitive enhancement. The challenge for future research is to seek out those conditions in which low serotonin significantly contributes to cognitive decline. Furthermore, future research aimed at clarifying the nature of the relationship serotonin and human cognition, identification of the role of 5-HT receptor subtypes, and the potential functional interactions between serotonin and other neurotransmitter systems that may underlie serotonin modulation of cognition, would be essential for understanding the full potential of 5HT drugs as cognition enhancers, even in healthy individuals. 


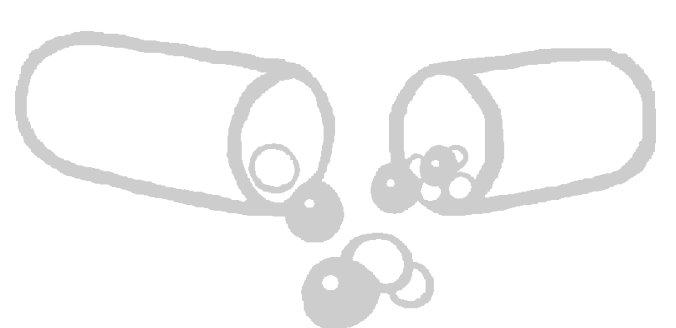

\title{
Chapter 3 \\ Actual driving performance and psychomotor function in healthy subjects after acute and subchronic treatment with escitalopram, mirtazapine and placebo
}

\begin{abstract}
Objective: The effects of escitalopram 10-20 mg/day and mirtazapine $30-45 \mathrm{mg} /$ day on actual driving and psychomotor performance of 18 healthy subjects were determined in a randomized, double-blind, placebocontrolled, multiple-dose, 3-way crossover trial. Method: Each treatment period lasted for 15 days and was separated from the next period by a washout period of at least 13 days. Subjects received an evening dose of escitalopram 10mg, mirtazapine 30mg, or placebo from Days 1-7 and an evening dose of escitalopram 20mg, mirtazapine 45mg, or placebo from Days 8-15. On Days 2, 9, and 16, reflecting acute period, dose increase, and steady state respectively, the Road Tracking test was performed. The main parameter was Standard Deviation of Lateral Position. Psychomotor performance was also assessed on Days 2, 9, and 16 by laboratory computer tasks. Subjective sleep quality was measured and mood was measured by visual analogue scales. Results: Treatment differences were apparent during the acute treatment period, in which subjects treated with mirtazapine $30 \mathrm{mg}$ performed less well in the driving test as compared to placebo. The Divided Attention Task results also revealed a significant increase in tracking error after a single dose of mirtazapine 30mg as compared to placebo. Mirtazapine decreased feelings of alertness and contentedness. Mirtazapine did not affect performance on days 9 and 16 of treatment. Escitalopram did not affect driving, psychomotor performance or subjective mood throughout treatment. Conclusion: In conclusion, driving performance, as well as psychomotor functioning, was not affected by escitalopram treatment in healthy subjects. Driving performance was significantly impaired after ingestion of mirtazapine $30 \mathrm{mg}$ during the acute treatment period.
\end{abstract}

Wingen, M., Bothmer, J., Langer, S., \& Ramaekers, J. G. (2005). Actual driving performance and psychomotor function in healthy subjects after acute and subchronic treatment with escitalopram, mirtazapine, and placebo: a crossover trial. Journal of clinical psychiatry, 66(4), 436-443. 


\section{Introduction}

Escitalopram is the S-enantiomer of citalopram and it is the most selective serotonin reuptake inhibitor (SSRI). Escitalopram treats major depressive disorder effectively (Lepola et al., 2003) and has been shown to be superior to citalopram (Auquier et al., 2003). The standard dose is 10 or $20 \mathrm{mg}$ daily and the most common side-effect is nausea (Aronson \& Delgado, 2004). Mirtazapine is a noradrenergic and specific serotonergic antidepressant (NaSSA) and its therapeutic effect is derived by blockade of the a2-adrenoceptors and by indirect stimulation of the $5-\mathrm{HT}_{1}$ receptors, via blockade of $5-\mathrm{HT}_{2}$ and $5-\mathrm{HT}_{3}$ receptors (De Boer \& Ruigt, 1995). Mirtazapine has shown antidepressant efficacy in placebo-controlled trials (Anttila \& Leinonen, 2001). The most prominent side-effects of mirtazapine are drowsiness or sedation, dry mouth, increased appetite, and weight gain (Kasper et al., 1997; Montgomery, 1995). Sedation is attributed to mirtazapine's high affinity for blocking the histaminergic H1 receptor (Anttila \& Leinonen, 2001). The sedative effects of mirtazapine may potentially last for a prolonged period, as the drug possesses an elimination half-life of 20-40 hours.

Antidepressants can have an impairing effect on psychomotor function and car driving due to side-effects, such as sedation, blurred vision or dizziness. These side effects may reduce the driving ability of depressed patients. However, not all antidepressants influence driving ability to the same extent. In cases of severe major depression, an effective antidepressant with few side effects, may even improve driving performance when alleviating the depression (Grappe et al., 1998; Hindmarch, 1995, 1997). Selective antidepressants, such as selective serotonin reuptake inhibitors (SSRIs) are known to have less impairing effects on car driving than tricyclic antidepressants (TCAs) in depressed patients (Brunnauer \& Laux, 2003). In addition, driving should be contraindicated during the starting phase of treatment with TCAs, because of the sedating effects that appear immediately after acute doses (Ramaekers, 2003). A few studies have determined the effects of citalopram or escitalopram on psychomotor performance and tasks related to driving performance in healthy volunteers. An acute dose of citalopram $10 \mathrm{mg}$ showed comparable effects to placebo on a number of psychomotor tests and on a driving simulator test (Wilkinson et al., 2003). Herberg (2001) (Herberg, 2001) found that citalopram 20 and $40 \mathrm{mg}$ daily did not impair psychomotor performance in healthy subjects. In another placebo-controlled study, an improvement in choice reaction time and critical flicker fusion threshold was shown, 1 to 4 hours after citalopram $20 \mathrm{mg}$ administration 
(Nathan et al., 2000). Other psychomotor tests showed no improvement, i.e. the digit symbol substitution test and the trailmaking B test (Nathan et al., 2000). After 1 and 8 days administration of 10, 20 and $40 \mathrm{mg}$ citalopram, there were no detrimental effects on psychomotor functioning, including the choice reaction time test and compensatory tracking, compared to placebo (Fairweather et al., 1997). In general, it can be said that citalopram is free of impairing psychomotor effects. There are some indications that citalopram as well as escitalopram in some degree decreases vigilance (Edgar et al., 2004; Riedel et al., 2005; Schmitt, Riedel et al., 2002). The relevance of this finding for actual driving performance is however presently unclear. No previous studies have been carried out to assess the effects of citalopram or escitalopram on actual driving performance.

The aim of the present study was to compare the influence of acute and subchronic treatment with escitalopram and mirtazapine on actual driving performance and psychomotor functioning in healthy subjects.

\section{Materials and methods}

\section{$\underline{\text { Subjects }}$}

Eighteen healthy subjects, 9 men and 9 women, mean age (sd): 31,4 (5,8), were recruited by advertisement in local newspapers. Subjects were screened by a telephone interview and a health questionnaire and all underwent a medical examination (including a standard 12- lead electrocardiogram, blood haematology and chemistry, urinalysis and drug and pregnancy screening). Selection was based on the following inclusion criteria: possession of a valid driving license for more than 3 years, driving experience of $>5000 \mathrm{~km}$ per year in average, normal binocular visual acuity corrected or uncorrected and body mass index between 19 to $29 \mathrm{~kg} / \mathrm{m}^{2}$. Subjects who met one or more of the following criteria were excluded from the study: history or present evidence of a serious illness such as renal, hepatic, cardiovascular, pulmonary, endocrine, neurological or psychiatric, haematological, gastrointestinal diseases, medical history of glaucoma, pregnancy (as determined at screening) or breastfeeding, known hypersensitivity to medicinal drugs, treatment with an investigational drug within 3 months prior to screening, use of medicines (except oral contraceptives and paracetamol), excessive smoking (more than 10 cigarettes a day), overconsumption of alcohol (more than 35g ethanol a day, comparable to 3,5 standard drinks) or caffeine (more than 6 cups of regular coffee a day), positive result 
of urine drug screening at the screening visit for alcohol and/or drug of abuse, positive result of $\mathrm{HCV}$ antibody or $\mathrm{HBs}$ antigen testing or blood donation.

The study was approved by the standing medical ethics committee of Maastricht University and it was carried out in accordance with the World Medical Association's Declaration of Helsinki (Edinburgh, 2000). Written informed consent was obtained from each subject prior to participation.

\section{Design and treatments}

The study was a randomized, double-blind, placebo-controlled, 3-way crossover design. Treatments were administered in separate 15-day series and treatment orders were balanced and assigned by a pre-determined randomization schedule. Subjects received $10 \mathrm{mg} /$ day escitalopram on Days 1 to 7 followed by $20 \mathrm{mg} / \mathrm{day}$ escitalopram on Days 8 to 15, $30 \mathrm{mg}$ /day mirtazapine on Days 1 to 7 followed by 45 $\mathrm{mg}$ /day mirtazapine on Days 8 to 15 or placebo. Drugs and placebo were always ingested at fixed times in the evening. Dosing started the evening before (Day 1) the first test day (Day 2).The treatment sessions were separated by washout periods of at least 13 days.

\section{Testing Procedure}

Subjects were trained in two sessions, one week prior to their first treatment condition in driving and psychometric tests to minimize learning effects. Training in the Critical Tracking Task and the Divided Attention task (see detailed descriptions below) continued until the subject had performed each test with less than 5\% variance from the average over the final three trials. The assessments were done on Days 2 (referred to as acute), Day 9 (dose increase) and Day 16 (steady state) of each treatment series, 12 to16 hours after drug administration. Subjects were not allowed to consume alcohol 48 hours prior to testing and caffeine-containing beverages 4 hours prior to testing. On each test day, subjects were screened for alcohol use in breath and for recent drug use in urine for opiates, methadone, cocaine, amphetamines, ecstasy and cannabinoids. During testing, subjects were not allowed to smoke. Subjects arrived at 9.00 a.m. and psychometric tests started at 9.30 a.m. The driving test started at 10.30 a.m. 
Actual Driving Performance (Road Tracking Test)

In the Road Tracking Test (O'Hanlon, 1984), subjects operated a specially instrumented vehicle over a $100 \mathrm{~km}$ primary highway circuit while maintaining a constant speed ( $95 \mathrm{~km}$ or 58 miles per hour) and a steady lateral position between the delineated boundaries of the right (slower) traffic lane. The vehicle was dualcontrolled and the subject was accompanied by a driving instructor. An electrooptical device mounted at the rear of the car continuously measured lateral distance separating the vehicle and the left lane-line. This signal was digitised at a rate of $4 \mathrm{~Hz}$ and stored on an onboard computer disk file for later editing analysis. The off line editing routine involved removal of all data segments that revealed signal loss, disturbance or occurrence of passing manoeuvres. The remaining data were then used to calculate means and variances for lateral position and speed (SP). Standard deviation of lateral position (SDLP in $\mathrm{cm}$ ) was taken as a variable. SDLP is a measure of road tracking error; in practical terms, a composite index of allowed weaving, swerving and overcorrecting. The standard deviation of the speed (SDSP) was also taken as a performance measure. The test duration was to be about 1 hour; the actual test duration varied between 45 and 120 minutes.

\section{Critical Tracking Task}

Critical Tracking Task (CTT) (Jex et al., 1966) measures the subject's ability to control a displayed error signal in a first-order compensatory tracking task. Error was displayed as an increasing horizontal deviation of a cursor from the midpoint on a horizontal, linear scale. Compensatory joystick movements nulled the error by returning the cursor to the midpoint. The frequency, at which the subject lost control, was the critical frequency or lambda ${ }_{c}\left(\lambda_{c}\right)$ in rad/s. The test included 5 trials of which the lowest and the highest scores were discarded; the average of the remaining scores was taken as the final score.

\section{Divided Attention Task}

Divided Attention Task (DAT) (Moskowitz, 1973) measures the ability to divide attention between two tasks performed simultaneously. Firstly, the subject performed the same tracking task as described above but at a constant level of difficulty set at $50 \%$ of his/her maximum capacity for 12 minutes. Tracking error is measured as the difference in millimetre between the position of the cursor and the midpoint of the scale. Secondly, the subject monitored 24 peripheral displays upon which single 
digits change asynchronously at 5 seconds intervals. The occurrence of the digit " 2 " was a signal for the subject to remove the foot from a pedal as rapidly as possible. Signals occurred twice at every location, in random order, at intervals of 5 to 25 seconds. Mean absolute tracking error (DATte in $\mathrm{mm}$ ) and average reaction time (DATrt in ms) were taken as variables.

\section{Syntactical Reasoning Task}

A series of 32 sentences were presented to the subject. Each described the order of the two letters; e.g. "B follows A". Each sentence is followed immediately by the same letters, printed on the computer screen; e.g. " $\mathrm{AB}^{\mathrm{N}}$. In half of the trials the order was the same as described by the preceding sentence and in the other half of the trials the order was opposite. Sentence difficulty varies within the series, from simple active sentences as given above to more complicated sentences involving passives, negatives or both; e.g., " $\mathrm{B}$ is not followed by $\mathrm{A}$ ". The required response was to indicate as quickly as possible using appropriate push buttons whether or not the pair of letters was in the same order as given in the preceding sentence. This task measures working memory (Baddeley, 1968). Correct number of responses (SRTcr) and mean reaction time (SRTrt) were the measurements.

\section{Digit-Symbol Substitution Task}

The Digit-Symbol Substitution Task (DSST) is a computerised version of the original paper and pencil test taken from the Wechsler Adult Intelligence Scale (Wechsler, 1981) and is a measurement of psychomotor speed, concentration and attention. The subject was briefly shown an encoding scheme consisting of a row of squares at the top of the screen, in which 9 digits were randomly associated with particular symbols. The same symbols were presented in a fixed sequence at the bottom of the screen as a row of separate response buttons. The randomization procedures were chosen such that symbols never appeared at the same ordinal position within both rows. The encoding scheme and the response buttons remained visible while the subject was shown successive presentations of a single digit at the centre of the screen. The task was to match each digit with a symbol from the encoding list and click the corresponding response button. The number of digits correctly encoded within 3 minutes was the performance measure (DSSTcr). 


\section{Subjective measurements}

Different visual analogue scales were used to assess subjective measurements of mood and drug effects on driving,. In addition, each test day subjects filled out the Groninger Sleep Quality Scale (Mulder-Hajonides van der Meulen, 1981) to assess sleep quality during the preceding night. The measurements were a total score of 14 yes/no questions to score the number of sleep complaints (ranging from good sleep (score 0) to worst possible sleep (score 14)) and specific questions about time needed to fall asleep, number of awakenings during the night and sleep duration in hours. Adverse events, observed, spontaneous reported by the subject or elicited upon a non-leading question were recorded. After the psychometric tests, subjects were asked to assess their mood by filling in a 16-item mood scale from which the factors Alertness, Contentedness and Calmness were derived (Bond \& Lader, 1974). Subjects were asked to rate their driving performance after the road tracking test and the driving instructor was asked to rate the driving performance and the degree of sedation of the subject after the road tracking test.

\section{Pharmacokinetics}

Blood samples for serum drug level analysis were collected on day 2, 9 and 16 of each treatment period using the following procedure: peripheral venous access was established and the required blood sample of $7 \mathrm{ml}$ whole blood was taken. The blood samples were analysed for serum concentration of escitalopram, the S-enantiomer and R-enantiomer of the metabolites demethylcitalopram (DCT) and didemethylcitalopram (DDCT), and for mirtazapine by means of a validated analysis method according to the principles of Good Laboratory Practice.

\section{Statistical analyses}

Sample size was based on a power calculation for detecting a treatment difference of $2.0 \mathrm{~cm}$ or more on the primary measure, i.e. Standard Deviation of Lateral Position (SDLP). A treatment difference of $2.4 \mathrm{~cm}$ was found to be clinically relevant which corresponds with a blood alcohol concentration (BAC) of $0.5 \mathrm{mg} / \mathrm{ml}$ in a study with social drinkers performing the same Road Tracking Test (Louwerens et al., 1987) The power of detecting a mean difference of $2.0 \mathrm{~cm}$ was calculated to be greater than $90 \%$ using the non-central $\mathrm{T}$ - distribution, with the within subject standard deviation being $2.1 \mathrm{~cm}$ as estimated in previous studies carried out by the Maastricht University research group. 
Parameters of the road tracking test, psychometric tests and subjective mood scales were subject to ANOVA analyses with Subject, Treatment and Period as factors. Driving and psychomotor data were analyzed for each test day separately. In case of a main treatment effect, drug-placebo effects were determined using simple contrasts. The results of the Groninger Sleep Quality scale were not normally distributed. To examine the main treatment effects, the variables were analyzed by the Friedman Test (nonparametric). In case of a significant treatment effect, drug-placebo comparisons were defined through the Wilcoxon signed-rank test (nonparametric). All statistical tests were conducted using SPSS (version 11.5 for Windows).

\section{Results}

\section{Missing data}

A total of 18 subjects completed the study. The road tracking test was interrupted by the driving instructor in one subject after treatment with mirtazapine $30 \mathrm{mg} /$ day (Day 2) because he fell asleep while driving. In this case, the remaining data (64\% complete) were used to calculate driving measurements. There were no missing data concerning the psychometric test. One subject did not complete the Groninger Sleep Quality scale Questionnaire during the second day of placebo treatment. These incomplete data were treated as missing values.

\section{Driving and psychometric tests}

Summary of the results of the driving test and the psychometric tests is shown in Table 1. Standard deviation of lateral position (SDLP) showed a significant effect of Treatment and Period $\left(\mathrm{F}_{2,32}=5.59, \mathrm{p}<0.05\right)$ on Day 2. Drug-placebo comparisons revealed an impairing effect of mirtazapine $(30 \mathrm{mg})$, but not of escitalopram $(10 \mathrm{mg})$. No interaction was found between Treatment and Period. Treatments did not affect SDLP on Days 9 and 16. Results of the SDLP are presented in Figure 1. Mean (se) tracking error in the divided attention task is presented in Figure 2 for each treatment condition. Significant effects of Treatment and Period $\left(F_{2,32}=3.46, p<0.05\right)$ were found on tracking error in the divided attention task on Day 2. Mirtazapine (30 mg) significantly increased tracking error, as demonstrated by drug-placebo contrasts. Escitalopram $(10 \mathrm{mg}$ ) had no effect on tracking error on Day 2 compared to placebo. No interaction was found between Treatment and Period. There were no significant Treatment effects demonstrated on Days 9 and 16. For the remaining performance measurements, no statistically significant effects of Treatment were established. 
Figure 1 Mean (SE) standard deviation of lateral position (SDLP) after 2, 9 and 16 days of treatment with escitalopram, mirtazapine and placebo

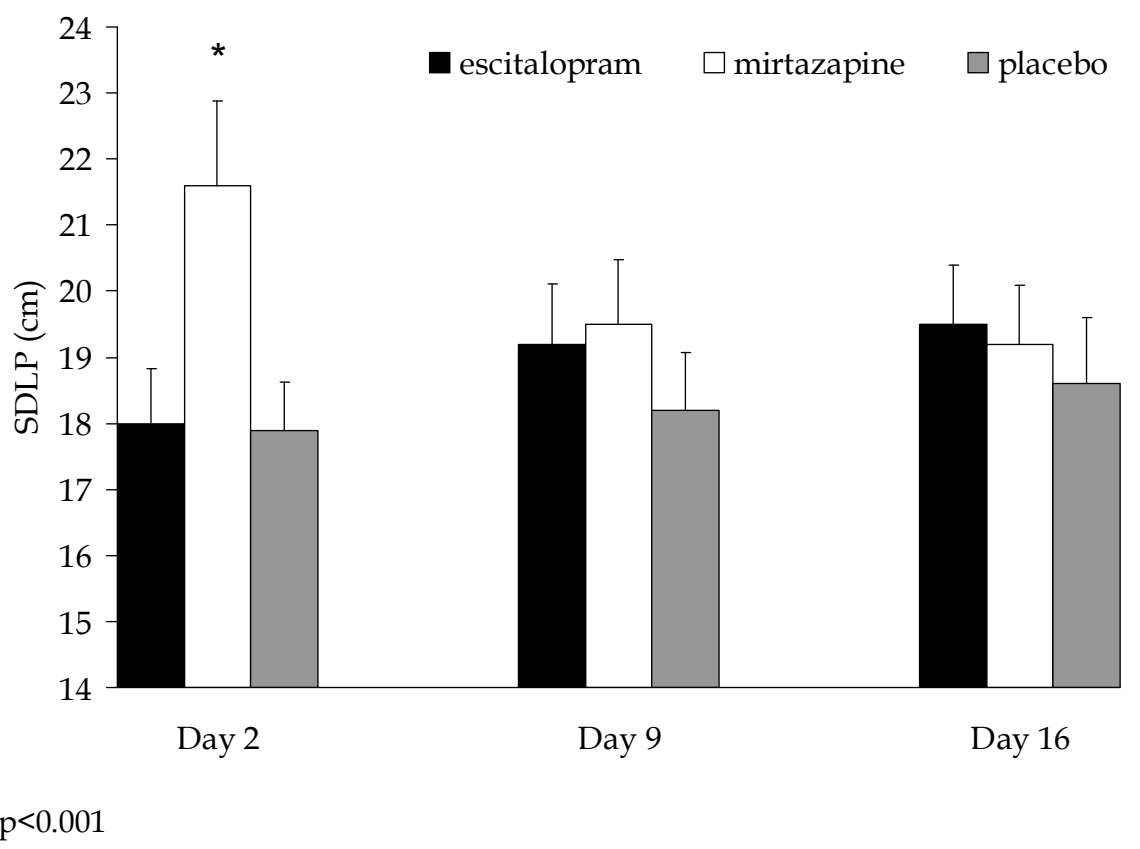

Figure 2 Mean (SE) tracking error of the divided attention task after 2, 9 and 16 days of treatment with escitalopram, mirtazapine and placebo.

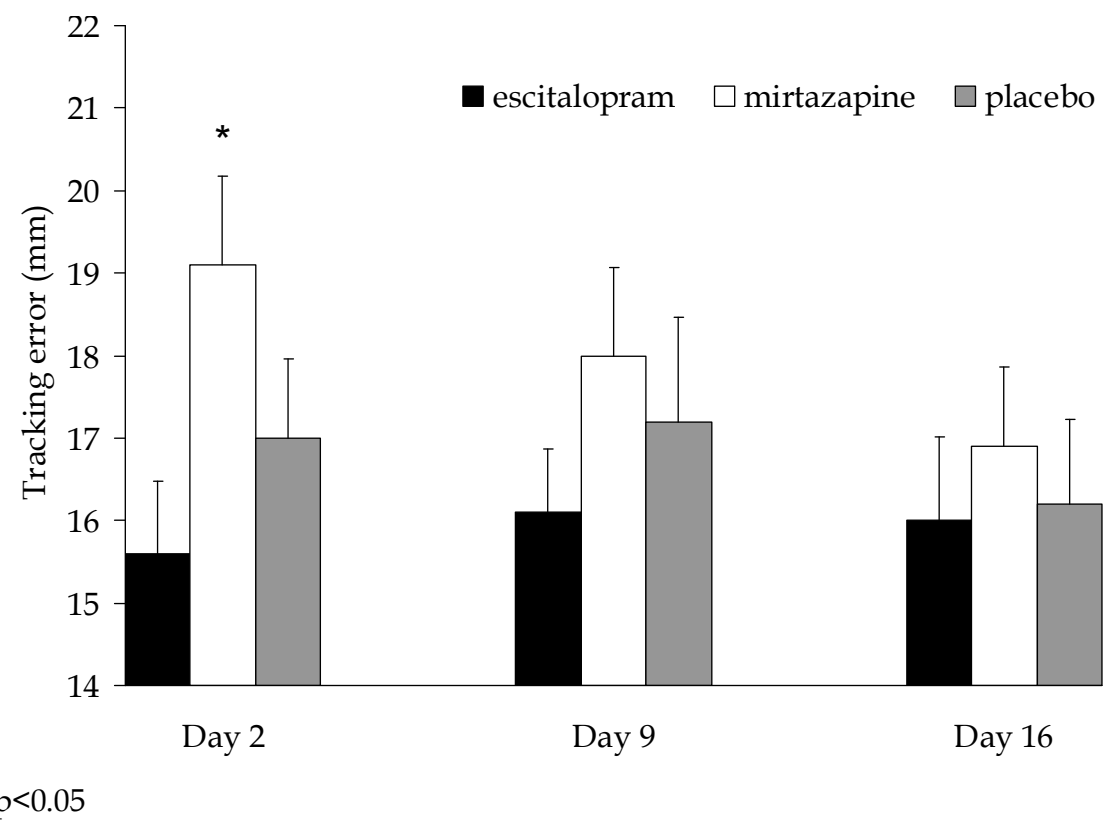




\section{Subjective measurements}

Summary of the results of subjective mood measurements are shown in Table 1. Alertness and Contentedness were significantly affected by Treatment on day 2 . Drug-placebo comparisons revealed that mirtazapine $(30 \mathrm{mg})$ reduced alertness and contentedness. No significant Treatment effects were demonstrated on day 2 for the factor calmness or on day 9 and 16 for the three factors.

Summary of the results of Groninger Sleep Quality Scale and subjective driving measurements are shown in Table 2. Friedman Test revealed effects of Treatment on sleep duration on nights 1 and 15. Drug-placebo comparisons showed that escitalopram significantly reduces sleep duration by 67 minutes, whereas mirtazapine increased sleep duration by 58 minutes during the first night of treatment. Other drug-placebo comparisons did not reveal significant differences.

Effects of Treatment on day 2 were significant for the ratings of driving performance by the driving instructor and by the subjects. Drug-placebo comparisons however revealed no significant treatment effects on driving performance rated by the driving instructor. Subjects rated their driving performance worse after mirtazapine (30 mg) treatment, demonstrated by placebo-drug comparisons. There was also a significant Treatment effect on day 9 on driving performance as rated by the subjects, but this could not be attributed to any of the drugs as compared to placebo.

\section{Adverse events}

The adverse events with the highest incidence in the escitalopram group were fatigue, insomnia and headache, followed by nausea (Table 3). In the mirtazapine group, the adverse event with the highest incidence was fatigue, followed by insomnia and headache. In the placebo group, fatigue, insomnia and headache occurred most frequently.

\section{Pharmacokinetics}

Mean (SD) plasma concentrations for escitalopram were 20.17 (4.60) nmol/L at Day 2, $71.44(27.89) \mathrm{nmol} / \mathrm{L}$ on Day 9 and 97.78 (41.46) nmol/L on Day 16. Mean (SD) plasma concentrations for mirtazapine were 67.67 (16.52) nmol/L on Day 2, 166.07 (52.64) nmol/L on Day 9 and 203.67 (98.48) nmol/L on Day 16. The values were within the expected therapeutic range. 
Table 1. Summary of the results of the driving test, psychometric tests and subjective mood measurements

\begin{tabular}{|c|c|c|c|c|c|c|c|c|c|}
\hline & \multirow[b]{3}{*}{ Day } & \multirow{2}{*}{\multicolumn{3}{|c|}{ Mean (standard error of mean) }} & \multirow{2}{*}{\multicolumn{3}{|c|}{$\begin{array}{l}\text { Treatment effect } \\
\text { Overall }\end{array}$}} & \multicolumn{2}{|c|}{ Contrast analyses } \\
\hline & & & & & & & & \multirow{2}{*}{$\begin{array}{c}\text { ESC vs PLA } \\
\mathrm{p} \\
\end{array}$} & \multirow{2}{*}{$\begin{array}{c}\text { MIR vs PLA } \\
\mathrm{p} \\
\end{array}$} \\
\hline & & PLA & ESC & MIR & $\mathbf{F}$ & df & $\mathrm{p}$ & & \\
\hline SDLP & 2 & $17.9(0.72)$ & $18.1(0.87)$ & $21.8(1.36)$ & 13.1 & 2,32 & $<.001$ & ns & $<.001$ \\
\hline \multirow[t]{2}{*}{$(\mathrm{cm})$} & 9 & $18.2(0.87)$ & $19.2(0.97)$ & $19.5(1.03)$ & 2.34 & 2,32 & ns & & \\
\hline & 16 & $18.6(1.01)$ & $19.4(0.94)$ & $19.2(0.94)$ & .503 & 2,23 & ns & & \\
\hline \multirow{3}{*}{$\begin{array}{l}\text { SDSP } \\
(\mathrm{km} / \mathrm{h})\end{array}$} & 2 & $1.68(0.09)$ & $1.61(0.08)$ & $1.68(0.09)$ & .470 & 2,32 & ns & & \\
\hline & 9 & $1.71(0.11)$ & $1.72(0.11)$ & $1.66(0.08)$ & .108 & 2,32 & ns & & \\
\hline & 16 & $1.68(0.82)$ & $1.76(0.08)$ & $1.68(0.07)$ & .578 & 2,32 & ns & & \\
\hline \multirow{3}{*}{$\begin{array}{l}\text { CTT } \\
(\mathrm{rad} / \mathrm{s})\end{array}$} & 2 & $4.17(0.18)$ & $4.20(0.18)$ & $3.96(0.17)$ & 2.60 & 2,32 & ns & & \\
\hline & 9 & $4.08(0.17)$ & $4.20(0.16)$ & $4.02(0.16)$ & 1.62 & 2,32 & $\mathrm{~ns}$ & & \\
\hline & 16 & $4.28(0.16)$ & $4.21(0.15)$ & $4.19(0.14)$ & .516 & 2,32 & ns & & \\
\hline \multirow{3}{*}{$\begin{array}{l}\text { DATte } \\
(\mathrm{mm})\end{array}$} & 2 & $17.0(0.96)$ & $15.5(0.91)$ & $19.1(1.14)$ & 7.65 & 2,32 & .002 & ns & .032 \\
\hline & 9 & $17.2(1.26)$ & $16.0(0.81)$ & $18.0(1.13)$ & 1.96 & 2,32 & ns & & \\
\hline & 16 & $16.2(1.03)$ & $16.0(1.07)$ & $17.0(1.01)$ & .749 & 2,32 & $\mathrm{~ns}$ & & \\
\hline \multirow{3}{*}{$\begin{array}{l}\text { DATrt } \\
\text { (ms) }\end{array}$} & 2 & $1683(79)$ & $1633(56)$ & $1717(60)$ & 1.12 & 2,32 & ns & & \\
\hline & 9 & $1727(80)$ & $1659(67)$ & $1671(63)$ & .687 & 2,32 & ns & & \\
\hline & 16 & $1646(59)$ & $1616(65)$ & $1612(53)$ & .233 & 2,32 & ns & & \\
\hline \multirow{3}{*}{$\begin{array}{l}\text { SRTcr } \\
(\#)\end{array}$} & 2 & $22.7(1.53)$ & $23.4(1.48)$ & $23.7(1.60)$ & .516 & 2,32 & ns & & \\
\hline & 9 & $24.0(1.63)$ & $24.3(1.32)$ & $23.6(1.50)$ & .348 & 2,32 & ns & & \\
\hline & 16 & $23.4(1.69)$ & $25.3(1.19)$ & $23.3(1.44)$ & 1.17 & 2,32 & ns & & \\
\hline \multirow{3}{*}{$\begin{array}{l}\text { SRTrt } \\
\text { (ms) }\end{array}$} & 2 & 1489 (94) & $1530(88)$ & $1613(85)$ & 2.27 & 2,32 & ns & & \\
\hline & 9 & 1417 (93) & $1474(98)$ & $1483(88)$ & .603 & 2,32 & ns & & \\
\hline & 16 & $1430(92)$ & $1463(81)$ & $1406(85)$ & 2.79 & 2,32 & ns & & \\
\hline \multirow{3}{*}{$\begin{array}{l}\text { DSSTcr } \\
(\#)\end{array}$} & 2 & $76.1(2.76)$ & $77.2(2.35)$ & $75.1(2.16)$ & .945 & 2,32 & ns & & \\
\hline & 9 & $78.1(2.88)$ & $78.9(2.45)$ & $77.0(1.92)$ & .629 & 2,32 & ns & & \\
\hline & 16 & $79.6(2.07)$ & $78.8(2.02)$ & $78.4(2.11)$ & .279 & 2,32 & ns & & \\
\hline \multirow{3}{*}{$\begin{array}{l}\text { Alertness } \\
(\mathrm{mm})\end{array}$} & 2 & $79.2(3.88)$ & $74.6(4.65)$ & $60.3(5.10)$ & 11.9 & 2,32 & $<.001$ & $\mathrm{~ns}$ & $<.001$ \\
\hline & 9 & $71.5(5.15)$ & $73.6(3.68)$ & $68.9(5.52)$ & .812 & 2,32 & ns & & \\
\hline & 16 & $76.9(5.78)$ & $76.0(4.28)$ & $71.3(4.53)$ & 1.70 & 2,32 & ns & & \\
\hline \multirow{3}{*}{$\begin{array}{l}\text { Conten- } \\
\text { tedness } \\
(\mathrm{mm})\end{array}$} & 2 & $85.3(3.42)$ & $82.7(3.55)$ & $80.5(3.51)$ & 4.24 & 2,32 & 0.023 & ns & .007 \\
\hline & 9 & $81.5(3.72)$ & $83.9(3.51)$ & $80.8(4.35)$ & 1.35 & 2,32 & ns & & \\
\hline & 16 & $84.9(3.54)$ & $84.8(3.94)$ & $83.6(3.80)$ & .235 & 2,32 & ns & & \\
\hline \multirow{3}{*}{$\begin{array}{l}\text { Calmness } \\
(\mathrm{mm})\end{array}$} & 2 & $83.7(4.06)$ & $80.1(4.68)$ & $83.1(4.14)$ & 3.05 & 2,32 & ns & & \\
\hline & 9 & $83.6(3.38)$ & $81.9(4.72)$ & $85.2(3.21)$ & .502 & 2,32 & ns & & \\
\hline & 16 & $87.8(2.54)$ & $81.9(4.58)$ & $82.3(3.68)$ & 1.74 & 2,32 & ns & & \\
\hline
\end{tabular}

Treatments are placebo (PLA), escitalopram 10-20 mg (ESC) and mirtazapine 30-45 $\mathrm{mg}(\mathrm{MIR}), \mathrm{df}=$ degrees of freedom, ns = not significant 
Table 2. Summary of the results of the Groninger Sleep Quality Scale and subjective driving measurement

\begin{tabular}{|c|c|c|c|c|c|c|c|c|c|}
\hline & \multirow[b]{3}{*}{ Night } & \multicolumn{3}{|c|}{ Mean (standard error of mean) } & \multicolumn{3}{|c|}{ Treatment effect } & \multicolumn{2}{|c|}{ Contrast analyses } \\
\hline & & \multirow[b]{2}{*}{ PLA } & \multirow[b]{2}{*}{ ESC } & \multirow[b]{2}{*}{ MIR } & \multicolumn{2}{|c|}{ Overall } & \multirow[b]{2}{*}{$\mathrm{p}$} & \multirow{2}{*}{$\begin{array}{c}\text { ESC vs PLA } \\
\mathrm{p} \\
\end{array}$} & \multirow{2}{*}{$\begin{array}{c}\text { MIR vs PLA } \\
\mathrm{p} \\
\end{array}$} \\
\hline & & & & & $\mathbf{F}$ & df & & & \\
\hline Sleep & 1 & $2.29(0.87)$ & $4.83(0.96)$ & $3.33(0.70)$ & 3.23 & 2,32 & ns & & \\
\hline \multirow[t]{2}{*}{ complaints } & 8 & $3.28(1.00)$ & $4.72(0.81)$ & $3.61(0.52)$ & 2.49 & 2,32 & ns & & \\
\hline & 15 & $3.56(0.88)$ & $4.61(0.99)$ & $2.72(0.49)$ & 2.27 & 2,23 & ns & & \\
\hline Time needed & 1 & $16.4(4.15)$ & $36.1(14.5)$ & $17.5(7.00)$ & 4.13 & 2,32 & ns & & \\
\hline to fall asleep & 8 & $17.6(4.23)$ & $15.4(3.09)$ & $25.0(7.62)$ & 0.13 & 2,32 & ns & & \\
\hline$(\mathrm{min})$ & 15 & $22.1(4.85)$ & $21.4(4.41)$ & $21.8(4.52)$ & 0.98 & 2,32 & ns & & \\
\hline \multirow{3}{*}{ \# Awakenings } & & $0.94(0.38)$ & $2.11(0.54)$ & $1.29(0.34)$ & 4.33 & 2,32 & ns & & \\
\hline & 8 & $1.44(0.33)$ & $1.44(0.35)$ & $1.17(0.39)$ & 2.72 & 2,32 & ns & & \\
\hline & 15 & $1.14(0.33)$ & $1.83(0.48)$ & $0.61(0.22)$ & 5.77 & 2,32 & ns & & \\
\hline \multicolumn{2}{|l|}{ Sleep duration 1} & $7.24(0.23)$ & $6.12(0.39)$ & $7.82(0.28)$ & 13.7 & 2,32 & .001 & \multirow[t]{3}{*}{.007} & \multirow[t]{3}{*}{.05} \\
\hline \multirow[t]{3}{*}{ (hours) } & 8 & $7.11(0.34)$ & $7.10(0.22)$ & $7.56(0.32)$ & 2.86 & 2,32 & ns & & \\
\hline & 15 & $7.28(0.32)$ & $6.76(0.24)$ & $7.78(0.22)$ & 12.76 & 2,32 & .002 & & \\
\hline & \multicolumn{4}{|l|}{ Day } & & & & & \\
\hline Driving rated & 2 & $77.4(4.62)$ & $71.3(4.79)$ & $12.3(53.7)$ & 6.37 & 2,32 & 0.041 & \multirow{3}{*}{$\begin{array}{l}\text { ns } \\
\text { ns }\end{array}$} & \multirow{3}{*}{$\begin{array}{c}0.025 \\
\text { ns }\end{array}$} \\
\hline by subject & 9 & $75.4(5.15)$ & $65.4(5.02)$ & $67.7(5.50)$ & 6.68 & 2,32 & \multirow{2}{*}{\begin{tabular}{|c|}
0.036 \\
$\mathrm{~ns}$
\end{tabular}} & & \\
\hline$(\mathrm{mm})$ & 16 & $71.9(5.6)$ & $63.0(3.89)$ & $66.3(5.09)$ & 3.44 & 2,32 & & & \\
\hline Driving rated & 2 & $81.1(2.23)$ & $79.2(2.69)$ & 74.8 (3.27) & 6.96 & 2,32 & 0.031 & \multirow[t]{3}{*}{ ns } & \multirow[t]{3}{*}{ ns } \\
\hline by instructor & 9 & $76.6(3.82)$ & $75.0(3.58)$ & $78.3(4.35)$ & 1.94 & 2,32 & \multirow{2}{*}{$\begin{array}{l}\text { ns } \\
\text { ns }\end{array}$} & & \\
\hline$(\mathrm{mm})$ & 16 & $77.1(3.06)$ & $71.3(4.75)$ & $74.4(3.47)$ & 1.53 & 2,32 & & & \\
\hline Sedation rated & & $19.5(6.01)$ & $20.9(6.70)$ & $28.2(7.16)$ & 1.34 & 2,32 & ns & & \\
\hline by instructor & 9 & $18.3(5.94)$ & $20.0(5.15)$ & $18.2(5.83)$ & 0.76 & 2,32 & ns & & \\
\hline$(\mathrm{mm})$ & 16 & $20.2(5.78)$ & $26.0(5.58)$ & $18.4(4.30)$ & 2.66 & 2,32 & ns & & \\
\hline
\end{tabular}

Treatments are placebo (PLA), escitalopram 10-20 mg (ESC) and mirtazapine 30-45 mg (MIR), $\mathrm{df}=\mathrm{degrees}$ of freedom, $\mathrm{ns}=$ not significant

Table3. Number and percentages of most common adverse events

\begin{tabular}{l||l|l|l}
\hline Preferred Term & PLA (n=18) & ESC (n=18) & MIR (n=18) \\
\hline \hline Fatigue & $3(16.7 \%)$ & $6(33.3 \%)$ & $11(61.1 \%)$ \\
Insomnia & $3(16.7 \%)$ & $4(22.2 \%)$ & $2(11.1 \%)$ \\
Somnolence & $1(5.56 \%)$ & $4(22.2 \%)$ & $7(38.9 \%)$ \\
Headache & $3(16.7 \%)$ & $4(22.2 \%)$ & $2(11.1 \%)$ \\
Dizziness & $1(5.56 \%)$ & $3(16.7 \%)$ & $5(27.8 \%)$ \\
Dry mouth & $1(5.56 \%)$ & $3(16.7 \%)$ & $1(5.56 \%)$ \\
Nausea & $2(11.1 \%)$ & $3(16.7 \%)$ & $1(5.56 \%)$ \\
Agitation & $0(0)$ & $1(5.56 \%)$ & $0(0)$ \\
\hline
\end{tabular}

Treatments are placebo (PLA), escitalopram 10-20 mg (ESC) and mirtazapine 30-45 mg 


\section{Discussion}

The main goal of the present study was to compare the effect of different evening doses of escitalopram and mirtazapine on actual driving performance as measured by the subjects' mean standard deviation of lateral position (SDLP) during the Road Tracking Test. Results from the present study showed that escitalopram did not affect driving performance in the acute $(10 \mathrm{mg})$, dose increase $(20 \mathrm{mg})$ or steady state treatment phase. Mirtazapine, on the other hand, produced driving impairment after the initial dose as indicated by a significant rise in mean SDLP compared to placebo. In the case of one subject the driving test could not be completed due to excessive sleepiness after a single dose of mirtazapine $30 \mathrm{mg}$. Mirtazapine's detrimental effect on driving decreased over time and was no longer of clinical relevance after repeated dosing. Escitalopram (10-20 mg) did also not affect psychomotor function after single and repeated doses. Mirtazapine impaired tracking in a divided attention task. This effect however was only apparent after a single dose and not after repeated drug administration. On the first night of treatment, subjective measurements of sleep showed a reduction of sleep duration after escitalopram administration and an increase of sleep duration after mirtazapine administration. In addition, subjective measurements of driving performance showed that subjects rated their driving performance much worse after mirtazapine $30 \mathrm{mg}$ treatment.

Results of the present study are fully in line with current notions regarding the effects of escitalopram and mirtazapine on psychomotor and/or driving performance. Escitalopram is a SSRI and SSRIs have generally been shown to produce no or little effect on psychomotor function and cognition. Mild psychomotor or cognitive impairment is most likely to occur for SSRIs possessing some affinity for muscarinic receptors such as paroxetine and fluvoxamine, or for a1 receptors such as nefazadone (see review: (Ramaekers, 2003)). Escitalopram, however, is the most selective SSRI available and possesses no affinity for additional receptors systems (see review: (Aronson \& Delgado, 2004)). The absence of any driving and psychomotor impairment in the present study provides further evidence that therapeutic doses of escitalopram do not affect driving performance.

Mirtazapine is an a2 antagonist that is known to possess strong, antagonistic binding affinities for postsynaptic serotonergic and histaminergic receptors. The antagonistic effect on histaminergic $\mathrm{H} 1$ receptors is not thought to mediate therapeutic effects. Rather H1 blockade causes somnolence and sedation that may result in performance impairment on a range of activities. The sedative effects of 
mirtazapine may potentially last for a prolonged period, as the drug possesses an elimination half-life of 20 to 40 hours. Consequently, mirtazapine is generally given at night to promote sleep and reduce daytime drowsiness.

Several investigations about the effects of mirtazapine on psychomotor function and driving in healthy volunteers have been reported. Ramaekers et al (1998) assessed the effect of evening doses of mirtazapine for 15 days. Actual driving and psychomotor assessments were conducted on days 2, 8, 9 and 16 of each period. Subjects received mirtazapine in doses of $15 \mathrm{mg}$ and $30 \mathrm{mg}$ nocte during the first and second week of dosing respectively. Mirtazapine 15mg nocte increased Standard Deviation of Lateral Position (the main driving parameter) by $2,2 \mathrm{~cm}$ after the first dose which was less than shown by social drinkers performing the same Road Tracking Test with a blood alcohol concentration (BAC) of $0.5 \mathrm{mg} / \mathrm{ml}$ (Louwerens et al., 1987). The magnitude of mirtazapine induced impairment was much less than those reported in other studies. Mattila et al (1989) (Mattila et al., 1989) for example, reported that single doses of mirtazapine $15 \mathrm{mg}$ and amitriptilyne $50 \mathrm{mg}$ produced severe and comparable psychomotor impairment after administration in the morning. The main difference with the study design of Ramaekers et al (1998) lies in the time of drug administration, namely at the test day or at the evening before the test day. Consequently, Ramaekers et al (1998) has suggested that the sedative effect of mirtazapine on daytime performance might be much alleviated by nocturnal administration. This notion has recently been confirmed by Ridout et al (Ridout et al., 2003) who assessed the psychomotor effects after evening and daytime doses of mirtazapine (15-30 mg) in a single, comparative placebo-controlled study over a 5 day period. Day time doses of mirtazapine $15 \mathrm{mg}$ significantly impaired performance in a Brake Reaction Time test on the first day of treatment. A single nocturnal dose of mirtazapine $15 \mathrm{mg}$ however did not affect brake reaction time after single and repeated doses. In addition, both drug regimens did not affect driving performance after 5 days of dosing. These studies thus seem to suggest that the impairing potential of mirtazapine in whole or in part is mitigated by nocturnal dosing or sleep. In addition, the degree of daytime performance impairment during mirtazapine treatment may also depend on the starting dose. The three studies above have assessed mirtazapine $15 \mathrm{mg}$ doses during treatment initiation, whereas in medical practice mirtazapine treatment is often started at higher doses, i.e. 30mg. Data from the present study thus may provide complementary information on the impairing 
effects of mirtazapine treatment on daytime performance, complementary during treatment initiation.

As it turned out in the present study study, an evening dose of mirtazapine $30 \mathrm{mg}$ significantly impaired actual driving performance and psychomotor function after the first night of treatment. The drug increased SDLP by $3.9 \mathrm{~cm}$ as compared to placebo in the Road Tracking Test. The effect of mirtazapine $30 \mathrm{mg}$ in the evening would be the equivalent of driving with a blood alcohol concentration (BAC) of above $0.5 \mathrm{mg} / \mathrm{ml}$ (Louwerens et al., 1987), i.e. the BAC above which drivers have an elevated risk of becoming involved in a traffic accident (Borkenstein et al., 1974). The rise in SDLP after the 30mg dose of mirtazapine was also bigger as compared to the effect of the noctural $15 \mathrm{mg}$ dose, i.e. 2,2, $\mathrm{cm}$ in the previous driving study (Ramaekers et al., 1998). The latter elevation in SDLP was associated with a BAC below $0.5 \mathrm{mg} / \mathrm{ml}$ and was generally considered of insufficient magnitude to reduce driver safety. The present data however demonstrate that the acute, sedative effect of mirtazapine on driving will become clinically relevant when treatment is started at higher doses, such as $30 \mathrm{mg}$ nocte.

The detrimental effect of mirtazapine on driving performance was primarily limited to the acute phase of treatment. There was some indication of a rise in SDLP after mirtazapine dose escalation, but this effect was relatively small in magnitude and not significantly different from mean SDLP in the escitalopram condition. Overall, driving performance did not differ during the dose escalation and steady state compared to placebo. Other measures also demonstrated that mirtazapine impairment was limited to the acute phase of treatment. Mirtazapine $30 \mathrm{mg}$ in the evening decreased tracking performance in a Divided Attention Task and decreased feelings of alertness and contentedness. The Critical Tracking task did not show impairment after mirtazapine $30 \mathrm{mg}$. Apparently, when the cognitive load performance is increased by adding a visual search task as in the Divided Attention Task, tracking performance becomes more vulnerable to the impairing effects of a drug. None of the effects were present after 1 or 2 weeks of repeated dosing. The absence of mirtazapine impairment after repeated dosing is probably related to the development of tolerance. Many studies have shown that tolerance to the acutely impairing effects of sedative antidepressants on driving performance develops within a few days of dosing (see review: (Ramaekers et al., 1998)). The implication is that driving under the influence of a sedative antidepressant such as mirtazapine should only be contraindicated during the acute phase of treatment. 
A potential limitation of the present study is the restricted age range of the subjects (21-40 years of age). It is generally believed that elderly people are more vulnerable to pharmacologic treatment. Generalisation of results from experimental driving studies in younger volunteers to the elderly population has to be done with caution (Ramaekers, 2003). The magnitude of driving impairment observed in adult volunteers might only be a conservative estimate of a drug's activity in elderly individuals who appear extra sensitive to pharmacologic treatment, particularly in case of sedating antidepressants.

In conclusion, escitalopram 10 to $20 \mathrm{mg}$ did not affect actual driving, psychomotor performance and cognitive function in healthy subjects. Mirtazapine 30mg nocte produced significant and clinically relevant impairment of driving and psychomotor performance during the acute treatment phase. The findings on psychomotor and driving performance are supported by subjective evaluations. Mirtazapine decreased feelings of alertness and contentedness; no subjective mood changes were present during escitalopram treatment. It is recommended to avoid high doses of mirtazapine during treatment initiation in order to promote safety over the day. The results from this study show that antidepressants can affect driving performance differently because of differences in their pharmacodynamic profiles. Importantly, broad class warnings about antidepressants and driving may not be informative enough and should be specified to individual substances.

\section{Acknowledgments}

This study was sponsored by H. Lundbeck A/S, Denmark. The results have previously been presented (as a poster) at the $25^{\text {th }}$ CINP congress, Paris, France June 20-24 2004 and at the 17th ECNP congress, Stockholm, Sweden, October 9-13 2004. We are thankful to A. van Oers, P. van Ruitenbeek, M. Klomp, J. de Hoogh and medical doctor C.J. van Leeuwen, for their assistance in the data collection. 


\title{
$Q_{\infty} \Omega$
}

Chapter 4

Driving impairment in depressed patients receiving long-term antidepressant treatment

\begin{abstract}
Background: Depression is a common mental disorder with cognitive deficits, but little information is available on the effects of antidepressant treatment on driving performance in depressed patients.

Aims: Assessing actual driving performance and cognition of depressed patients receiving long-term antidepressant treatment. Method: Performance was assessed in depressed patients receiving SSRI (Selective Serotonin Reuptake inhibitor) or SNRI (Serotonin and Noradrenalin Reuptake Inhibitor) treatment for 6-52 weeks and in matched, healthy controls by means of two standardized on-the-road driving tests and laboratory tests of cognition. Results: Data showed poorer driving performance as indicated by a higher Standard Deviation of Lateral Position (SDLP) or 'weaving motion' in medicated patients relative to controls. Time to speed adaptation and Critical Flicker Fusion Threshold were also impaired in medicated patients. Hamilton Depression Rating Scale scores in medicated patients were significantly higher as compared to controls. No other significant results between the two groups were demonstrated on the variables of the driving tests and laboratory tests of cognition. Conclusions: Depressed patients receiving long-term treatment with SSRI and SNRI type antidepressants show impaired driving performance. This impairment in driving performance can probably be attributed to residual depressive symptoms instead the of antidepressant treatment.
\end{abstract}

Wingen, M., Ramaekers, J. G., \& Schmitt, J. A. (2006). Driving impairment in depressed patients receiving long-term antidepressant treatment. Psychopharmacology, 188(1), 84-91. 


\section{Introduction}

Depression is a common mental disorder that, in addition to affective disturbances, is characterized by loss of energy and cognitive decline (Austin et al., 2001; Burt et al., 1995; Zakzanis et al., 1998). This pattern of symptoms is likely to affect daily functions, including driving ability. However, despite concerns of both patients and clinicians regarding driving ability during depression, driving performance of treated or untreated depressed patients remains poorly investigated.

Epidemiological data show that depression itself and the use of sedative antidepressants increase the relative risk of depressed patients of becoming involved in traffic accidents (Leveille et al., 1994; Ray et al., 1992; Vaa, 2003). On the other hand, selective serotonin reuptake inhibitors and related antidepressants did not increase crash risk in patients (Barbone et al., 1998). Experimental driving studies have shown that sedative antidepressants such as TCAs and alpha-2-antagonists produce driving impairment through 1-2 weeks after treatment initiation (Ramaekers, 2003). However, these experimental driving studies have mainly focused on measuring antidepressant effects in healthy volunteers rather than in depressed patients. Only one study assessed the effects of antidepressant treatment with fluoxetine and moclobemide on actual on actual road driving performance in a depressed patient population (Ramaekers et al., 1997). That study showed that driving performance did not improve over a 6 week course of treatment with any of these antidepressants and that driving was not correlated to changes in HAMD ratings.

Information on the long term effects of antidepressant drugs on driving in depressed patients is presently not available. In general, it can be expected that nonsedating antidepressants that are prescribed nowadays produce few side effects and will produce little or no driving impairment and possibly may even improve driving performance in depressed patients by alleviating the depressive symptoms. However, this has never been investigated.

In the present study, the effect of long-term antidepressant treatment in depressed patients on actual driving performance and cognition was examined. Driving performance of depressed patients receiving chronic antidepressant treatment and healthy controls was assessed in two on-the-road driving tests in normal traffic. Cognition was measured by tasks that are related to driving skills and tasks that are shown to be sensitive to impaired performance associated with depression i.e. memory and attention measurements. It was expected that driving 
performance and cognition of depressed patients receiving long-term treatment with non-sedating antidepressants was comparable to that of matched healthy controls.

\section{Materials and Methods}

\section{Subjects}

Twenty-four depressed patients who received antidepressant treatment with a SSRI or venlafaxine for 6-52 weeks, referred to as treated patients, were recruited through regional psychiatric centers in the South-Limburg area and by advertisement in local newspapers. In addition, twenty-four healthy volunteers were recruited by advertisement in local newspapers. Participants were screened by a telephone interview and health questionnaire including questions about the individual's medical condition and medical history, lifestyle and demographical characteristics.

All participants were free from neurological, cardiovascular, respiratory, metabolic, hepatic or renal disorders or a history of these illnesses, did not use illicit drugs, were not pregnant or lactating and were devoid of any sensory or motor deficits that could reasonably be expected to affect test performance. All participants had a valid driving license for at least three years and driving experience of at least $5000 \mathrm{~km} /$ years during each of the preceding three years.

Healthy participants were free from psychiatric illnesses at present or in the past and had no first degree relative with a history of a psychiatric illness. Treated patients had a primary diagnosis of unipolar disorder with an active depressive episode by a psychiatrist or physician according to DSM-IV criteria with scores on the Hamilton Depression Rating Scale above 17. In addition, the diagnosis prior to start of treatment was confirmed by a retrospective Structured Clinical Interview for DSMIV (SCID) at the laboratory. None of the participants had a present or past diagnosis of psychotic depression.

Healthy participants did not take any medication, except for oral contraceptives. Depressed patients received treatment with citalopram $(n=4$, average dose: $25 \mathrm{mg}$ (SD 10), range: 20-40 mg), sertraline ( $\mathrm{n}=4$, average dose: 87,5 mg (SD 25 ), range: $50-100 \mathrm{mg})$, paroxetine ( $\mathrm{n}=8$, average dose: $28,8 \mathrm{mg}$ (SD 9.9), range: $20-40 \mathrm{mg}$ ) or venlafaxine ( $\mathrm{n}=8$, average dose: $134,4 \mathrm{mg}$ (SD 75.5), range: $75-300 \mathrm{mg})$, but no other psychoactive medication. Two patients used concomitant antihypertensive medication, i.e irbesartan $150 \mathrm{mg}$ and lisinopil $20 \mathrm{mg}$. Demographical characteristics of the two groups are shown in table 1. The study was approved by the standing medical ethics committee of Maastricht University and the Maastricht Academic 
Hospital's Board of Directors. It was carried out in accordance with the World Medical Association's Declaration of Helsinki (Edinburgh, 2000). Written informed consent was obtained from each volunteer prior to participation to the study.

Table 1: Subject characteristics for the group of patients receiving antidepressant treatment for 6-52 weeks, their matched healthy controls.

\begin{tabular}{l||l|l}
\hline Characteristics & $\begin{array}{l}\text { Depressed patients with } \\
\text { treatment for 6-52 weeks }\end{array}$ & Healthy Volunteers \\
\hline \hline $\mathbf{N}$ & 24 & 24 \\
\hline Sex & $12(50 \%)$ & $12(50 \%)$ \\
$\quad$ Male & $12(50 \%)$ & $12(50 \%)$ \\
Female & 42.2 & 41.8 \\
\hline Age & 21 & 22 \\
Mean & 63 & 61 \\
Minimum & & \\
Maximum & $8.8(\mathrm{SE} 1.37)$ & $0.5(\mathrm{SE} 0.19)$ \\
\hline Hamilton Depression rating Scale & 0 & 0 \\
Mean & 26 & 3 \\
Minimum & $19(79 \%)$ & - \\
Maximum & 0 & - \\
\hline Precipitating factor & $5(21 \%)$ & - \\
None & 0 & - \\
Somatic illness & $3(13 \%)$ & - \\
Psychosomatic stressors & $21(87 \%)$ & - \\
Both & 0 & - \\
\hline Characterization & 0 & - \\
Depression with anxiety & 0 & \\
Depression with mainly & & \\
somatic symptoms & & \\
Retarded depression & & \\
Neurotic depression & & \\
\hline
\end{tabular}

\section{$\underline{\text { Design }}$}

The study was conducted according to a parallel group design, comparing a group of treated depressed patients with healthy controls. Each patient was matched to a healthy control subject comparable for age, gender and years of driving experience.

\section{Testing Procedure}

On a separate training day, participants practiced the driving and cognitive tests to familiarize with the procedure and test instructions. On the test day, participants arrived at the university laboratory at $13.15 \mathrm{~h}$ and subsequently filled out the selfrating questionnaires. At $13.30 \mathrm{~h}$ the cognitive test battery was administered, which took approximately 35 minutes. Next, the Hamilton Depression Rating Scale was completed. At $14.30 \mathrm{~h}$ participants were driven to the starting point of the driving circuit. During the subsequent 105 minutes the driving assessments were conducted, 
allowing for a 10-minute break between the tests. The participants arrived back at the university at $17.15 \mathrm{~h}$. In total, the test day lasted 4 hours.

\section{Assessments}

Actual driving performance

\section{Road Tracking Test}

In the Road Tracking Test (O'Hanlon, 1984), participants operated a specially instrumented vehicle over a $100 \mathrm{~km}$ primary highway circuit while maintaining a constant speed (95 km/58 miles per hour) and a steady lateral position between the delineated boundaries of the right (slower) traffic lane. The vehicle was dualcontrolled and the participant was accompanied by a driving instructor. An electrooptical device mounted at the rear back of the car continuously measured lateral distance separating the vehicle and the left lane-line. This signal was stored on an onboard computer disk file for later editing analysis. The remaining data were used to calculate means and variances for lateral position and speed. Standard deviation of lateral position (SDLP in $\mathrm{cm}$ ) was taken as a performance measure. SDLP is a measure of road tracking error; in practical terms, a composite index of weaving, swerving and overcorrecting. The standard deviation of the speed was also taken as a performance measure.

\section{Car-following Test}

The car following test, adapted from a previous study (Brookhuis et al., 1994), involved two cars driving on a secondary highway (Ramaekers et al., 2002). The participant controlled the following car accompanied by a driving instructor. The participant was requested to attempt to drive at 15-30 meters behind the preceding car which was controlled by a second driving instructor. The test began with the two vehicles traveling one after the other at speeds of $70 \mathrm{~km} / \mathrm{h}$. The speed of the leading car was automatically controlled by a modified cruise-control system and could be changed within amplitude of 10 kilometers per hour. The main parameter was the time to speed adaptation (TSA in $\mathrm{msec}$ ); this is the reaction time of the participant to speed and deceleration of the leading car. Between speed maneuvers, the investigator in the leading car randomly activated the brake lights. The participant was instructed to react to the brake lights by removing his/her foot from the speed pedal as fast as possible; this parameter was called brake reaction time. Headway was continuously recorded. 


\section{Cognition}

The cognitive test battery consisted of various tasks measuring psychomotor and cognitive function. The visual verbal learning task, an adapted version of the Auditory Verbal Learning Task, was used to assess short and long term memory function (Rey, 1964). The Change Blindness Task measured visual scene analysis and memory. Participants were shown a series of 100 photographs from various traffic situations on a computer screen. Subjects were required to report changes in each of these traffic scenes that occurred during an occlusion period of $1 \mathrm{sec}$ (Dornhoefer et al., 2002). The Left-Right test was used as a parametric version of the well-known color-word response conflict task. This test measures simple reaction time and selective attention (Stroop, 1935). The Continuous Performance Test (CPT) was used to study vigilance (attention) (Conners, 1992)and also provided a measure of the ability to inhibit or suppress a habit response. In the present study a computerized version of the original CPT was used. The Critical Flicker Fusion Threshold was determined using the Leeds Psychomotor Tester (Leeds Psychomotor Services, York). This is a measure of CNS arousal and activation (Curran \& Wattis, 1998; Schmitt, Riedel et al., 2002). The mean of six measurements was taken as dependent variable. Finally, a computerized version of the Digit-Symbol Substitution Task (DSST) (Wechsler, 1981)was administered.

\section{Subjective measurements}

The Hamilton Depression Rating Scale (Hamilton, 1967) and Beck's Depression Inventory (Beck et al., 1961) were used to rate vegetative and cognitive symptoms of depression, including co morbid anxiety symptoms. In addition, participants rated feelings of depression, tension, fatigue, vigor and anger by means of the Profile Of Mood States (McNair et al., 1992). Participants filled out the Groninger Sleep Quality Scale on the evenings prior to testing in order to assess subjective sleep quality and sleep duration (Mulder-Hajonides van der Meulen, 1981). Participants as well as the driving instructor were asked to rate their driving performance after completion of the driving tests.

\section{Statistical analyses}

The outcome variables of the driving tests, cognitive tests and questionnaires were subject to analysis of variance (ANOVA) comparing two groups: patients treated with antidepressants and matched healthy controls. Subsequent analysis on type of 
antidepressant (i.e. SSRI vs SNRI) was conducted on outcome variables that showed a significant difference between patients and controls. Differences in performance between SSRI treated patients and their matched healthy controls were compared to differences in performance between venlafaxine treated patients and their matched healthy controls, also by ANOVA to examine if type of antidepressant has an influence on the results. A correlational analysis of HDRS scores and SDLP was conducted over difference scores of treated patients and their matched healthy controls. All statistical tests were conducted using SPSS (version 11.5 for Windows).

\section{$\underline{\text { Results }}$}

Actual Driving Performance

Road Tracking Test

ANOVA revealed a statistically significant difference in SDLP between patients and controls. SDLP was higher for treated patients compared to healthy controls $(\mathrm{F} 1,46=8.29, \mathrm{p}<0.01)$. Results of SDLP for patients and controls are presented in Figure 1.

\section{Car-following Test}

Time to speed adaptation in the car following test was statistically significant impaired in treated patients compared to healthy controls $(F 1,46=5.73, p<0.05)$ presented in Figure 2. Other outcome measures of this test, i.e. brake reaction time, speed and headway did not differ significantly between groups. Means (SE) of these measures are shown in table 2. Means (SE) of these measures are shown in table 2.

\section{Cognition}

Average Critical Flicker Fusion threshold frequency was statistically significantly reduced in treated patients compared to healthy controls $(F 1,46=11.16, p<0.01)$. For the remaining cognitive performance measurements, no statistically significant effects were established. Means (SE) of all cognitive assessments are shown in table 2. 
Figure 1. Mean (SE) Standard Deviation of Lateral Position (SDLP)

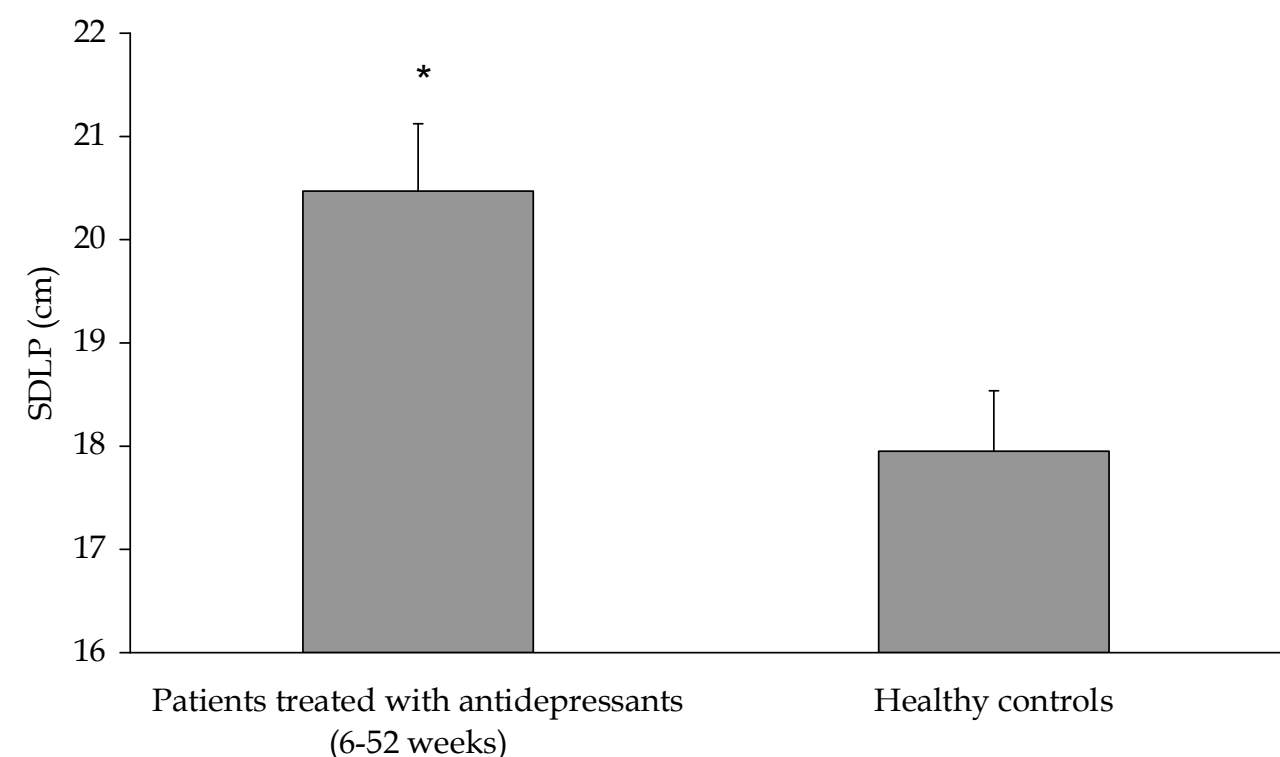

${ }^{*} \mathrm{p}<0.01$

Figure 2. Mean (SE) Time to Speed Adaptation (TSA) of the car following test

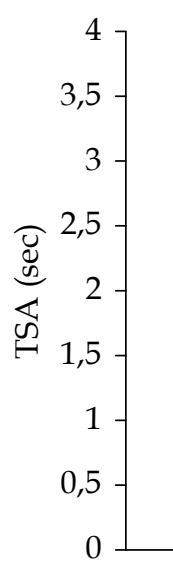

Patients treated with antidepressants (6-52 weeks)

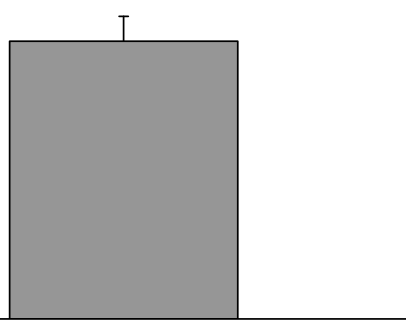

Healthy controls

${ }^{*} \mathrm{p}<0.05$ 
Table 2: Cognitive and driving assessments (means, SE) for the group of depressed patients receiving antidepressant treatment for 6 to 52 weeks and healthy controls.

\begin{tabular}{|c|c|c|}
\hline Assessments/outcome measures & $\begin{array}{l}\text { Depressed patients with } \\
\text { treatment for 6-52 weeks }\end{array}$ & Healthy Volunteers \\
\hline \multicolumn{3}{|l|}{ Driving Assessments } \\
\hline \multicolumn{3}{|l|}{ Road Tracking Test } \\
\hline Standard Deviation of Lateral Position & $20.5(0.65)$ & $18.0(0.58)$ \\
\hline$(\mathrm{cm})$ & $96.2(0.22)$ & $95.8(0.24)$ \\
\hline Speed & $2.2(0.30)$ & $1.9(0.36)$ \\
\hline SD Speed & & \\
\hline Car Following Test & $588(21.7)$ & $548(28.1)$ \\
\hline Brake reaction time (msec) & $3.1(0.33)$ & $2.1(0.19)$ \\
\hline Time To Speed Association (sec) & & \\
\hline Driving Instructor Rating & $6.6(0.31)$ & $7.4(0.28)$ \\
\hline Driving quality & $1.1(0.41)$ & $0.49(0.19)$ \\
\hline 'Sedation' & & \\
\hline $\begin{array}{c}\text { Volunteer Rating } \\
\text { Driving quality } \\
\end{array}$ & $3.9(0.37)$ & $2.8(0.25)$ \\
\hline \multicolumn{3}{|l|}{ Cognitive Tests } \\
\hline \multicolumn{3}{|l|}{30 Word Learning Test } \\
\hline Immediate recall (\# words) & $35.0(2.1)$ & $35.2(1.9)$ \\
\hline Delayed recall (\# words) & $11.8(0.90)$ & $10.9(0.91)$ \\
\hline Delayed recognition (\% correct) & 80.3 & 80.7 \\
\hline Delayed recognition speed (msec) & $800(34.8)$ & $828(24.0)$ \\
\hline \multicolumn{3}{|l|}{ Left-Right Task } \\
\hline Compatible RT (msec) & $604(16.6)$ & $614(13.2)$ \\
\hline Incompatible RT (msec) & $628(11.6)$ & $635(13.1)$ \\
\hline Incompatible correct responses & $15.9(0.06)$ & $15.8(0.12)$ \\
\hline Compatible correct responses & $15.6(0.10)$ & $15.7(0.12)$ \\
\hline \multicolumn{3}{|l|}{ Critical Flicker Fusion } \\
\hline Threshold frequency $(\mathrm{Hz})$ & $19.8(0.42)$ & $22.0(0.50)$ \\
\hline \multicolumn{3}{|l|}{ Digit Symbol Substitution Task } \\
\hline \multicolumn{3}{|l|}{ Continuous Performance Test } \\
\hline Reaction time hits (msec) & 384 (11.6) & $385(9.94)$ \\
\hline Correct detections (\#) & $46.0(0.73)$ & $47.0(0.27)$ \\
\hline \multicolumn{3}{|l|}{ Change Blindness Task } \\
\hline Overall correct detections & $43.1(1.36)$ & $44.7(1.24)$ \\
\hline Relevant correct detections & $29.8(0.72)$ & $31.1(0.63)$ \\
\hline Irrelevant correct detections & $14.2(1.00)$ & $13.6(0.77)$ \\
\hline RT overall correct detections (msec) & $884(31.3)$ & $871(28.4)$ \\
\hline RT relevant correct detections (msec) & $780(46.8)$ & $807(28.8)$ \\
\hline RT irrelevant correct detections (msec) & $1032(43.6)$ & $1018(42.3)$ \\
\hline
\end{tabular}

\section{Subjective measurements}

A summary of the results of subjective mood measurements is shown in Table 3. There was a statistically significant difference in scores on the Hamilton Depression Rating Scale between the two groups. Patients treated with antidepressants had a higher HDRS score (F1,46=35,49, p<0.001).

Treated patients had statistically significant higher scores on the Beck Depression Inventory as compared to healthy controls $(F 1,46=27.2, p<0.001)$. 
Furthermore, treated patients had statistically significant higher scores on the 'depression' $(\mathrm{F} 1,46=11.2, \mathrm{p}<0.05)$, 'tension' $(\mathrm{F} 1,46=10.1, \mathrm{p}<0.05)$ and 'fatigue' (F1,46=14.6, $\mathrm{p}<0.001)$ subscales of the POMS, and significantly lower scores on the 'vigour' subscale $(\mathrm{F} 1,46=21.4, \mathrm{p}<0.001)$. No effects were seen on the 'anger' subscale. Sleep quality was statistically significant reduced in treated patients. The average number of sleep complaints in these patients was more than 3 times as high as those reported by healthy volunteers $(\mathrm{F} 1,46=11.3, \mathrm{p}<0.01)$. The level of alertness and driving quality, as rated by the accompanying driving instructor, of treated patients was not statistically significant different than that of healthy controls. However, treated patients' rating of their own driving ability was statistically significant worse than that of healthy controls $(\mathrm{F} 1,46=6.81, \mathrm{p}<0.05)$.

Table 3: Subjective assessments of mood and sleep quality (means, SE) for the group of depressed patients receiving antidepressant treatment for 6 to 52 weeks and healthy controls.

\begin{tabular}{l||l|l}
\hline Questionnaire/ subscale & $\begin{array}{l}\text { Depressed patients with } \\
\text { treatment for 6-52 weeks }\end{array}$ & Healthy Volunteers \\
\hline \hline Beck Depression Inventory (BDI) & $11.8(1.8)$ & $3.0(0.5)$ \\
\hline Profile of Mood States (POMS) & & \\
$\quad$ Depression & $51.9(2.9)$ & $63.7(2.0)$ \\
$\quad$ Tension & $34.2(2.5)$ & $47.1(1.3)$ \\
Fatigue & $34.23(2.8)$ & $41.0(1.8)$ \\
$\quad$ Vigour & $79.4(1.9)$ & $90.6(1.5)$ \\
$\quad$ Anger & $51.60(2.7)$ & $54.27(1.5)$ \\
\hline Sleep Quality & & \\
$\quad$ Number of sleep complaints & $5.2(0.9)$ & \\
(Groninger sleep questionnaire) & & \\
\hline
\end{tabular}

\section{Type of antidepressant}

Given the different types antidepressants i.e. SSRI and venlafaxine, it was investigated whether these subtypes affected outcomes variables differently. Outcome variables including SDLP, TSA, average Critical Flicker Fusion threshold frequency, HDRS, POMS and average number of sleep complaints revealed no statistically significant differences on type of antidepressant. The only statistically significant difference was shown on the Beck Depression Inventory $(F 1,22=6,19$, $p<0.05)$ with higher difference scores between venlafaxine treated patients and their matched healthy controls compared to SSRI treated patients. 


\section{Correlation analysis}

There was no statistically significant correlation between difference scores of HDRS and difference scores of SDLP. No statistically significant differences were revealed by dividing treated patients in 2 groups of 'low' (HDRS<8) and 'high' HDRS scores on SDLP. The mean SDLP for these groups were 20,14 $(n=13)$ and 20,86 $(n=11)$ respectively.

\section{Discussion}

This is the first study that examined the effects of long-term treatment with SSRI and SNRI antidepressants on actual driving performance in depressed patients. Results revealed a statistically significant impairment of driving performance in depressed patients receiving long-term antidepressant treatment as compared to healthy controls. Driving impairment was evident from increments in weaving (SDLP) and time to speed adaptation (TSA) on the on-the-road driving tests. Objective impairment in the driving test was corroborated by subjective ratings of driving performance. Patients receiving long-term antidepressant treatment rated their own driving ability significantly worse as compared to healthy controls. No significant differences between depressed patients receiving antidepressant treatment as compared to healthy controls were found on cognition such as memory and attention. Driving performance was comparable in patients receiving SSRIs or venlafaxine.

Previous studies also suggest that successful antidepressant treatment may not normalize driving ability of depressed patients. In depressed patients receiving amitriptyline, little improvement was seen on a series of laboratory tasks aimed at assessing fitness for driving in the first six weeks of treatment, although depression ratings fell significantly (Gerhard \& Hobi, 1984). Even after treatment with novel antidepressants depressed patients showed an impairing effect on a 'act and react' test system assessing driving skills, although these novel antidepressants have been shown to produce less performance impairment than tricyclic antidepressants (Brunnauer \& Laux, 2003). In another study, 89\% of depressed patients receiving polypharmacological antidepressant treatment assessed with the same 'act and react' test system, were considered unfit to drive (Grabe et al., 1998). The validity of these laboratory tasks in terms of real life driving performance remains uncertain but the results are confirmed by one study using an actual on the road driving test in depressed patients. Here an improvement of depressive symptoms but no improvement of SDLP in depressed patients treated with fluoxetine and 
moclobemide during 6 weeks of treatment was demonstrated (Ramaekers et al., 1997).

It is very difficult to validate the results of the driving tests against the criterion of actual accident involvement, but it is possible to quantify the test results against a secondary criterion that itself is highly correlated with accident risk, i.e. blood alcohol concentration (BAC). The association between SDLP and BACs between 0.00 and $0.15 \mathrm{mg} / \mathrm{mL}$ has been established in a series of controlled studies with healthy social drinkers (Louwerens et al., 1987). Against these data, the increase in SDLP in the treated patients receiving SSRI treatment would compare to a BAC of $0.50 \mathrm{mg} / \mathrm{ml}$, which is the legal BAC limit for driving in many countries. It is important to note that the comparisons with alcohol merely illustrate the magnitude of the SDLP effects; they do not necessarily translate into comparable accident risks. It is unclear to which extend the factors that determine SDLP performance also contribute to the increased accident risk with alcohol.

The absence of deficits in memory, psychomotor and attention functions in the medicated depressed patient group in the present study is consistent with the notion that successful SSRI therapy is generally associated with an alleviation of the cognitive deficits that are seen during a depressive episode (Amado-Boccara et al., 1995). However, driving performance appears to be much less susceptible to antidepressant treatment. Our observation of a decreased CFF threshold - an indirect measure of central arousal (Curran, 1990) - in the medicated patients in our study suggests that impaired driving performance in this group may be related to suboptimal energetic resources, perhaps due to the mild reduction in sleep quality that was found in this group. One may argue that the prolonged driving tests are more sensitive to arousal-mediated effects on performance than short-lasting laboratory tests. Correlational analysis has shown that conventional psychomotor and cognitive tests do not strongly predict antidepressant effects on actual driving performance (Ramaekers, 2003). Residual depressive symptoms were present in the treated depressed patients group (average HDRS of 8.8 versus 0.5 in healthy controls) and these may possibly be associated to impaired driving performance. However, no correlation was found between driving performance and HDRS, suggesting that depressive symptomology is not a primary factor in the observed effects. Further, although citalopram (Riedel et al., 2005), paroxetine (Schmitt, Ramaekers et al., 2002), and venlafaxine (O'Hanlon et al., 1998) have been shown to induce mild reductions of vigilance in healthy volunteer studies, these drugs generally do not affect SDLP after 
acute or repeated doses in healthy volunteers (Ramaekers, 2003). It is therefore unlikely that behavioral side-effects of the SSRIs influenced driving performance in a meaningful way. Rather driving impairment in depressed patients seems most likely caused by the residual depression itself.

While antidepressant treatment apparently did not normalize driving performance of depressed patients, it may still have attenuated driving impairment relative to untreated depression. We did not test untreated depressed patients, but in order to gain insight into this issue, we compared our data to historical SDLP data obtained from baseline assessments of a study on the effects of fluoxetine and moclobemide on driving performance (Ramaekers et al., 1997). The SDLP methodology was identical except for the type of vehicle used. To control for this, we calculated a correction factor based on the difference between mean (placebo) values obtained in 8 studies with the previous car and 8 studies with the current car. Also, we were able to match a sub-sample of the untreated patients to our patients, based on age, gender and driving experience. The corrected SDLP in untreated depressed patients was 21.9 (SE 1.1) compared to 20.5 (SE 0.7) in the present treated patient group. There was no statistically significant difference between both groups. Nevertheless, the average increase in SDLP seen in the untreated patients was reduced by $35 \%$ in the SSRI and venlafaxine treated patients, suggesting there may be a small beneficial effect of treatment on driving performance. It is clear that the use of historical controls may have many pitfalls and that changes in driving performance over the course of antidepressant treatment need to be studied directly in order to obtain a reliable picture.

In conclusion, it was shown that depressed patients receiving long-term SSRI or SNRI treatment exhibit statistically significant inferior driving skills, as assessed with standardized on the road driving tests, compared to matched healthy controls. Based on the fact that there are generally no detrimental effects of SSRIs on driving performance and despite no correlation between HDRS scores and SDLP, the impairment in driving performance can probably be attributed to residual depressive symptoms but this remains to be investigated. Whether the observed impairment translates into increased accident risk remains to be established, but it seems prudent that patients are advised of potentially compromised driving ability, even when clinical remission by antidepressant treatment is achieved. Further research, specifically longitudinal studies addressing the time course of the effects of various antidepressant treatments on driving performance in depressed patients, is needed to 
elucidate the relation between depression, antidepressant treatment and driving ability.

\section{Acknowledgments}

The authors are thankful to drs Debije, Geeraerts, Meijer, Havermans, Peeters and Van Boxtel for recruiting and selecting the patients, and Marije Meeuwissen, Nadine Kessen, Michiel Schraven, Irma Brauers and Henk Brauers for their work during data collection. This study was conducted as a part of the IMMORTAL project which was funded by the EU $5^{\text {th }}$ Framework Programme (http://www.immortal.or.at). Additional financial support was received from the Hersenstichting Nederland. 


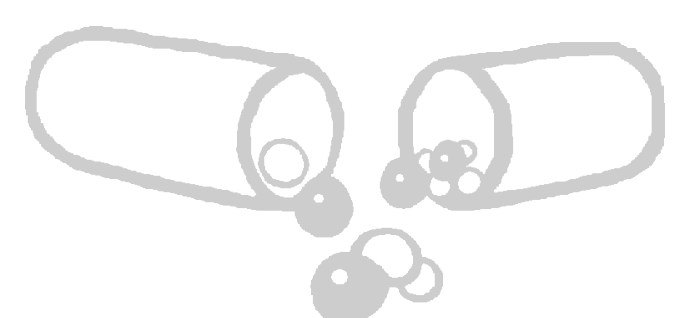

\title{
Chapter 5 \\ Verbal Memory performance during subchronic challenge with a selective serotonergic and a mixed action antidepressant
}

\begin{abstract}
Objectives: Effects of escitalopram 10-20 mg/day and mirtazapine $30-45 \mathrm{mg} /$ day on verbal memory of 18 healthy participants were assessed in a randomized, double-blind, placebocontrolled, 3-way crossover trial.

Method: Each treatment period lasted for 15 days and was separated from the next period by a washout period of at least 13 days. Participants received an evening dose of escitalopram $10 \mathrm{mg}$, mirtazapine $30 \mathrm{mg}$, or placebo from days $1-7$ and an evening dose of escitalopram $20 \mathrm{mg}$, mirtazapine $45 \mathrm{mg}$, or placebo from days $8-15$. On days 2 , 9, and 16 a visual verbal memory task was performed measusing drug effects during the acute phase, after dose increase and at steady state.

Results: Escitalopram did not affect immediate or delayed verbal memory score throughout treatment. During mirtazapine treatment, participants performed less well in the overall immediate recall score compared to placebo. This impairment was most pronounced in the final trial of the visual verbal learning task.

Conclusion: Verbal memory was not affected by acute and subchronic escitalopram treatment in healthy participants. Overall immediate verbal memory was slightly but significantly impaired throughout mirtazapine treatment, probably due to a general reduction in overall arousal caused by $\mathrm{H} 1$ blockade.
\end{abstract}

Wingen M, Langer S, Ramaekers JG (2006): Verbal memory performance during subchronic challenge with a selective serotonergic and a mixed action antidepressant. Human psychopharmacology 21:473-9. 


\section{Introduction}

The role of serotonin in cognition and specifically in memory is an issue of present interest. Recent studies have given new insights in serotonergic manipulations and memory. Animal research shows that serotonin receptors are situated in brain areas involved in memory and different 5-HT-agonist or 5-HT-antagonists show memory improvement or impairment (Buhot et al., 2000; Meneses, 1999). There are a number of ways to examine the role of serotonin in memory in healthy volunteers. A convincing way to study the effect of decreased serotonin levels is tryptophan depletion. Several studies show an impairment of memory consolidation after acute tryptophan depletion in healthy volunteers (Riedel et al., 2002) which implicates that lowered serotonin levels are associated with memory impairment. Conversely, one could expect that serotonin augmentation would improve verbal memory.

Increased serotonin levels in healthy volunteers can be obtained by the use of antidepressants, particularly by the use of selective serotonin reuptake inhibitors (SSRIs). A few studies in healthy volunteers have been conducted to assess the effects of SSRIs on memory specifically but these studies have shown controversial results. Subchronic administration of sertraline produced no effects on memory compared to placebo (Schmitt et al., 2001; Siepmann et al., 2003) but subchronic administration of paroxetine did impair delayed recall on a memory task (Schmitt et al., 2001). An acute dose of the SSRI fluvoxamine, 50 and $100 \mathrm{mg}$, produced no effect on memory compared to placebo (Fairweather et al., 1996). Different doses of citalopram had comparable effects to placebo on the Sternberg Memory Scanning task after acute and subchronic (8 days) administration in healthy volunteers (Fairweather et al., 1997). Administration of $40 \mathrm{mg} /$ day citalopram for 15 days impaired delayed recall compared to placebo, whereas $100 \mathrm{mg} /$ day sertraline did not (Riedel et al., 2005). There was just one study showing an improvement of delayed verbal memory after increased serotonin levels caused by SSRI administration (Harmer et al., 2002). In the latter study, citalopram $10 \mathrm{mg}$ was administered intravenously which probably resulted in higher brain serotonin levels as compared to oral dosing studies. The controversial results of the above-mentioned studies could be due to differences between specific SSRIs. For instance, the impairing effect of paroxetine is probably attributional to additional muscarinic receptor blockade, next to paroxetines' serotonergic mechanism (Schmitt et al., 2001). Other differences between the results may have resulted from methodological differences such as a variety in memory tasks, in SSRI dosages or in time of treatment administration. 
The present study tried to take these differences into account and assessed the effect of different dosages of escitalopram and mirtazapine on short term and long term verbal memory. Escitalopram is the S-enantiomer of citalopram. Escitalopram is currently the most selective serotonin reuptake inhibitor (Owens et al., 2001; Sanchez et al., 2004). It therefore may serve as a valuable tool in challenge studies of the serotonergic system. It was hypothesized that an acute and subchronic dose of escitalopram may enhance verbal memory. In this study, the noradrenergic and specific serotonergic antidepressant (NaSSA) mirtazapine was used as an active control. Due to its high affinity for blocking the histaminergic $\mathrm{H} 1$ receptor (Anttila \& Leinonen, 2001; Biswas et al., 2003) mirtazapine shows sedative properties. Acute and subchronic treatment with mirtazapine was hypothesized to impair memory.

\section{Materials and Methods}

\section{Subjects}

Eighteen healthy participants ( 9 women and 9 men) with a mean age (SD) of $31,4(5,8)$ were recruited by advertisement in local newspapers. All participants underwent a medical examination by a medical doctor, including a standard 12- lead electrocardiogram, blood haematology and chemistry, urinalysis, drug and pregnancy screening. In addition, participants filled out an extensive medical questionnaire, which was reviewed by the medical doctor. Exclusion criteria were history or present evidence of renal, hepatic, cardiovascular, pulmonary, endocrine, neurological or psychiatric illness, haematological, gastrointestinal diseases and medical history of glaucoma. Other exclusion criteria were body mass index below 19 or above $29 \mathrm{~kg} / \mathrm{m}^{2}$, pregnancy (as determined at screening) or breastfeeding, known hypersensitivity to medicinal drugs, treatment with an investigational drug within 3 months prior to screening, use of medicines (except oral contraceptives and paracetamol), excessive smoking (more than 10 cigarettes a day), overconsumption of alcohol (more than 35mg ethanol a day) or overconsumption of caffeine (more than 6 cups of regular coffee a day), positive result of urine drug screening at the screening visit for alcohol and/or drug of abuse, and any sensory or motor deficits which would reasonably be expected to affect test performance. All participants gave their written informed consent prior to participation. The study was approved by the standing medical ethics committee of Maastricht University and it was carried out in accordance with the World Medical Association's Declaration of Helsinki (Edinburgh, 2000). 


\section{Design and treatments}

The study was conducted according to a randomized, double-blind, placebocontrolled, 3-way crossover design. Treatments were administered in separate 15-day series and treatment orders were balanced and assigned by a Latin square design resulting in 6 treatment orders over 18 participants. Participants received $10 \mathrm{mg} /$ day escitalopram at days 1 to 7 followed by $20 \mathrm{mg}$ /day escitalopram at days 8 to 15,30 $\mathrm{mg}$ /day mirtazapine at days 1 to 7 followed by $45 \mathrm{mg} /$ day mirtazapine at days 8 to 15 or placebo. Drugs and placebo were always ingested in a given time-frame in the evening (between 8.00 and 10.00 p.m.). Dosing (day 1) started in the evening prior to the first test day (day 2). The treatment sessions were separated by washout periods of at least 13 days.

\section{Testing Procedure}

Participants were trained prior to the study to practice the memory test. The assessments were done at days 2, 9 and 16 of each treatment series, 12-14 hours after drug administration. Participants were not allowed to consume alcohol 48 hours prior to testing and caffeine-containing beverages 4 hours prior to testing. During testing, participants were not allowed to smoke. Participants completed the Visual Verbal learning task (see below) at the training session and at every test day. There were ten assessments ( 1 training session and 3 testdays per each of the 3 treatment periods). Therefore ten versions of the visual verbal learning task, with different but comparable words, were used. This study was a part of a larger study previously published (Wingen et al., 2005) in which participants were subjected to an extensive testing battery.

\section{Visual verbal learning task}

The visual verbal learning task is an adapted version of the Auditory Verbal Learning Task and assesses short and long term memory function (Rey, 1964). The test consists of a list of 15 monosyllabic words. Each word is shown on the computer display for 2 seconds and the participant is requested to read it aloud and to remember it. The participant is asked to freely recall as many words as possible when the series ends. The number of words remembered is the first trial score. In total, the same list is presented in the same manner for five successive occasions. The words correctly recalled in all five trials are summed to the total immediate recall score, a measure for 
short-term memory. After 30 minutes delay with distracting tasks to prevent the participant from rehearsing the learned words, the participant is asked to recall as many words as possible. The number of words correctly recalled is taken as the delayed recall score, a measure for long-term memory. This is followed by a recognition test, where 15 words from the list previously shown and 15 other monosyllabic words are presented. The participant has to respond as quickly as possible by pressing the "YES" or "NO" button to indicate recognition of the word. The number of correctly recognized words counts for the delayed recognition score.

\section{$\underline{\text { Statistical analyses }}$}

All statistical tests were conducted using SPSS (version 11.5 for Windows). Parameters of the visual verbal learning task were subject to univariat ANOVA analyses. Memory data were analyzed overall with Subject, Treatment, Day and Period as factors and for each test day separately with Subject, Treatment and Period as factors. In case of a Treatment effect, drug-placebo effects were determined using simple contrasts. The alpha criterion significance level was set at $\mathrm{p}=0.05$.

\section{Pharmacokinetics}

Blood samples for drug serum level analysis were collected on day 2, 9 and 16 of each treatment period using the following procedure: peripheral venous access was established and the required blood sample of $7 \mathrm{ml}$ whole blood was taken. The blood samples were analysed for serum concentration of escitalopram, the S-enantiomer and R-enantiomer of the metabolites demethylcitalopram (DCT) and didemethylcitalopram (DDCT), and for mirtazapine by means of a validated analysis method according to the principles of Good Laboratory Practice.

\section{$\underline{\text { Results }}$}

All 18 participants completed the study. Mean (SD) plasma concentrations for escitalopram were 20.17 (4.60) nmol/L at Day 2, 71.44 (27.89) nmol/L at Day 9 and 97.78 (41.46) nmol/L at Day 16. Mean (SD) plasma concentrations for mirtazapine were 67.67 (16.52) nmol/L at Day 2, 166.07 (52.64) nmol/L at Day 9 and 203.67 (98.48) $\mathrm{nmol} / \mathrm{L}$ at Day 16. The values were within the expected therapeutic range. 


\section{Immediate recall}

Overall analysis revealed a significant effect on the total immediate recall score on the factors Treatment $\left(\mathrm{F}_{2,138}=3,03, \mathrm{p}=0.05\right)$, Day $\left(\mathrm{F}_{2,138}=6,10, \mathrm{p}<0.01\right)$ and Period $\left(\mathrm{F}_{2,138}=4,24\right.$, $\mathrm{p}<0.05)$. Further analyses showed no significant Treatment by Day or Treatment by Period interaction. This indicates that the treatment effect stands for its own. According to drug-placebo comparisons, mirtazapine had an impairing effect on total immediate recall. Escitalopram did not impair total immediate recall (see Figure 1). Subsequently, the overall analysis revealed a significant Treatment effect $\left(F_{2,138}=3,41\right.$, $\mathrm{p}<0.05)$ on the fifth trial score. Drug-placebo comparisons showed an impairing effect of mirtazapine $(p=0.017)$. A significant Period effect was found on the third trial score $\left(\mathrm{F}_{2,138}=4,48, \mathrm{p}<0.05\right)$ but there was no Drug by Period interaction. A summary of the results of the Visual Verbal Learning task is shown in table 1. Analyzing each day separately, there were no Treatment effects on Day 2, 9 or 16 shown in table 2.

Figure 1. Means (SE) of theVisual Verbal Learning Task - overall total immediate recall score.

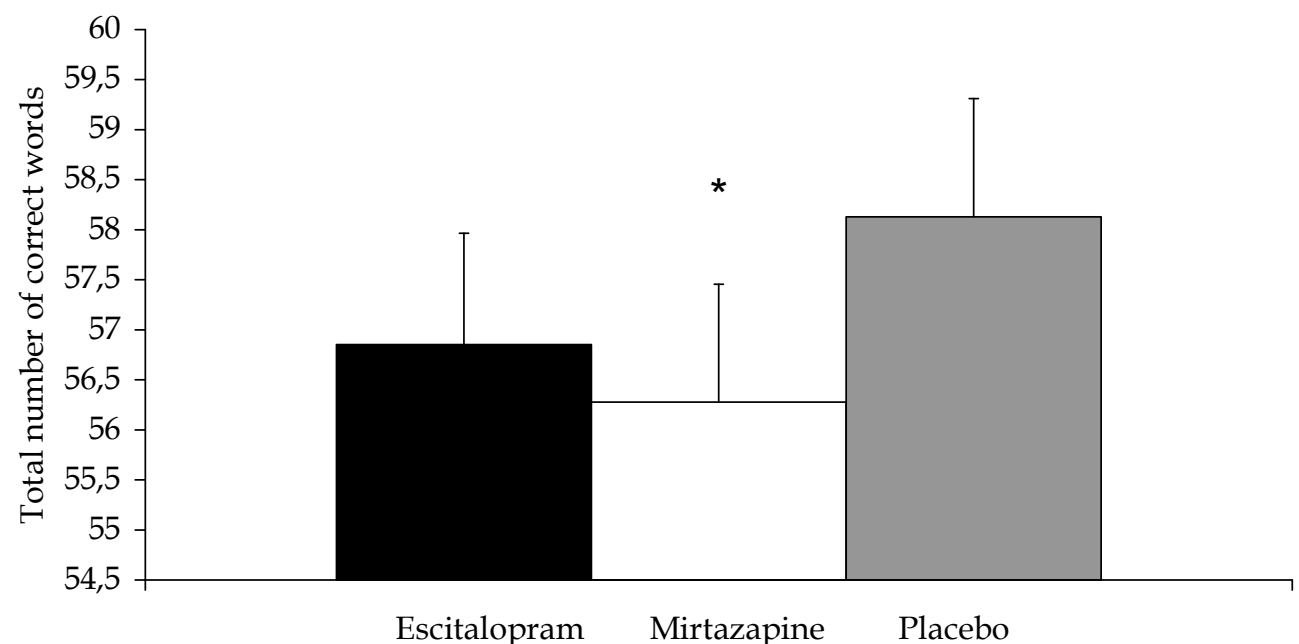

${ }^{*} \mathrm{p}=.017$ mirtazapine compared to placebo

\section{Delayed recall}

No significant effects on the delayed recall score or on the delayed recognition score were detected, overall and for each day separately. 
Table 1. Summary of the overall average results for each trial of the Visual Verbal Learning Task

\begin{tabular}{l||l|l|l|l|l|l}
\hline \multirow{2}{*}{ Measurement } & \multicolumn{2}{l}{ Mean (SE) of number of words } \\
\cline { 2 - 7 } & Escitalopram & Mirtazapine & Placebo & F & df & p \\
\hline \hline Immediate recall & & & & & & \\
\hline Trial 1 & $7,7(0,30)$ & $7,9(0,31)$ & $8,0(0,32)$ & 0,7 & 2,138 & $\mathrm{~ns}$ \\
\hline Trial 2 & $10,7(0,29)$ & $10,6(0,31)$ & $11,1(0,31)$ & 2,0 & 2,138 & $\mathrm{~ns}$ \\
\hline Trial 3 & $12,1(0,25)$ & $12,1(0,26)$ & $12,6(0,28)$ & 2,3 & 2,138 & $\mathrm{~ns}$ \\
\hline Trial 4 & $13,0(0,25)$ & $12,7(0,23)$ & $13,0(0,24)$ & 0,7 & 2,138 & $\mathrm{~ns}$ \\
\hline Trial 5 & $13,4(0,24)$ & $13,0(0,24)$ & $13,6(0,21)$ & 3,4 & 2,138 & .036 \\
\hline Delayed recall & $12,1(0,35)$ & $11,7(0,37)$ & $12,1(0,39)$ & 1,2 & 2,138 & $\mathrm{~ns}$ \\
\hline Word recognition & & & & & & \\
\hline Correct & $28,7(0,19)$ & $28,5(0,24)$ & $28,8(0,20)$ & 0,5 & 2,138 & $\mathrm{~ns}$ \\
\hline Reaction time & $650(6,61)$ & $648(7,4)$ & $651(7,5)$ & 0,1 & 2,138 & $\mathrm{~ns}$ \\
\hline
\end{tabular}

ns= not significant

Table 2. Summary of the results for each test day of the Visual Verbal Learning Task

\begin{tabular}{|c|c|c|c|c|c|c|c|}
\hline \multirow{2}{*}{\multicolumn{2}{|c|}{ Measurement }} & \multicolumn{6}{|c|}{ Mean (SE) of number of words } \\
\hline & & Escitalopram & Mirtazapine & Placebo & $\mathbf{F}$ & $\mathrm{df}$ & $\mathrm{p}$ \\
\hline \multicolumn{8}{|c|}{ Immediate recall } \\
\hline & Day 2 & $55,3(2,0)$ & $55,1(1,94)$ & $56,7(2,31)$ & 0,9 & 2,32 & ns \\
\hline & Day 9 & $56,2(2,08)$ & $56,7(2,25)$ & $58,6(2,04)$ & 1,9 & 2,32 & ns \\
\hline & Day 16 & $59,1(1,68)$ & $57,1(2,00)$ & $59,1(1,84)$ & 2,1 & 2,32 & ns \\
\hline \multicolumn{8}{|c|}{ Delayed recall } \\
\hline & Day 2 & $12,2(0,53)$ & $11,5(0,60)$ & $12,4(0,69)$ & 1,7 & 2,32 & ns \\
\hline & Day 9 & $11,6(0,65)$ & $11,4(0,70)$ & $12,2(0,68)$ & 1,0 & 2,32 & ns \\
\hline & Day 16 & $12,3(0,62)$ & $12,1(0,65)$ & $11,8(0,67)$ & 0,5 & 2,32 & ns \\
\hline \multicolumn{8}{|c|}{ Word recognition } \\
\hline \multirow[t]{3}{*}{ Correct } & Day 2 & $28,9(0,34)$ & $28,6(0,37)$ & $28,7(0,39)$ & 0,3 & 2,32 & ns \\
\hline & Day 9 & $28,7(0,29)$ & $28,4(0,50)$ & $28,9(0,32)$ & 0,6 & 2,32 & ns \\
\hline & Day 16 & $28,6(0,37)$ & $28,6(0,38)$ & $28,7(0,32)$ & 0,01 & 2,32 & ns \\
\hline \multirow[t]{3}{*}{$\mathrm{RT}(\mathrm{msec})$} & Day 2 & $660(11,90)$ & $658(12,39)$ & $663(16,30)$ & 0,1 & 2,32 & ns \\
\hline & Day 9 & $640(9,59)$ & $642(11,58)$ & $639(10,62)$ & 0,03 & 2,32 & ns \\
\hline & Day 16 & $649(12,77)$ & $645(14,71)$ & $652(11,29)$ & 0,2 & 2,32 & ns \\
\hline
\end{tabular}

ns= not significant

\section{Discussion}

The results of this study suggest that different dosages (10mg and 20mg) of escitalopram have no effect on short-term and long-term verbal memory whereas mirtazapine (30mg increased up to $45 \mathrm{mg}$ after 8 days) slightly impaired immediate recall, during 16 days of treatment compared to placebo. The impairing effect was particularly evident in the fifth and final learning trial. The impairing effect of mirtazapine on memory was rather small and only reached statistical significance when combined over all treatment days.

It was hypothesized that escitalopram would enhance memory by increasing central serotonin levels. For citalopram an improving effect on verbal memory, specifically delayed recall and recognition, had been shown (Harmer et al., 2002). There are several reasons that can account for acute escitalopram administration not 
having enhancing effects in the present study. Harmer et al. (2002) administered citalopram $10 \mathrm{mg}$ intravenously, however in the present study escitalopram $10 \mathrm{mg}$ was administered orally. The estimate of bioavailability for escitalopram (oral administration) appears to be around 80\% (Sogaard et al., 2005). Although limited, first-pass metabolism of the hepatic circulation may decrease the blood plasma levels for escitalopram. As a result, the serotonin levels in the brain may be lower after oral administration compared to intravenous administration. Furthermore, the time of drug administration differed between both studies. Harmer et al (2002) used daily dosing and the memory task was carried out $45 \mathrm{~min}$ after the end of the infusion. In the present study, escitalopram was administered in the evening and verbal memory was tested the next day. Consequently, there was no measurement of the earliest action of escitalopram. Still, the results of the present study are in line with the results of a previous study on escitalopram $(10 \mathrm{mg})$ in which no effect on verbal memory was found after acute treatment (Wilkinson et al., 2003). Future studies on serotonin and memory should emphasize the use of intravenous administration of SSRI's for manipulating the serotonergic system although this may move away from the clinical understanding of the cognitive and behavioural toxicity of oral medication.

The present study found no effects on verbal memory after subchronic administration of escitalopram. Previous studies showed that subchronic administration of citalopram did not affect memory (Fairweather et al., 1997), but 15 days of administration of citalopram $40 \mathrm{mg}$ impaired delayed recall (Riedel et al., 2005). Time of drug administration can be a possible explanation for the rather deviant results in the present study. Daytime testing after daytime administration (46 hours after citalopram intake) may substantially differ from daytime testing after evening administration (12-14 hours after escitalopram intake). The possibility exist that overnight sleep after nocturnal administration may have mitigated any detrimental effects of escitalopram on memory performance. For example, previous studies have show that daytime driving impairment after treatment with sedating antidepressant was much less after nocturnal dosing as compared to daytime dosing even though drug-blood concentration were the same after both dosing regimes (Ramaekers, 2003).

The role of serotonin in memory is still unknown. Only one study showed memory improving effects after increasing serotonin by means of a SSRI (Harmer et al., 2002). Probably, the serotonergic system is a very complex system including many different serotonergic receptors. As animal studies showed, stimulation of different 5- 
HT receptors can have opposing effects on memory (Buhot et al., 2000; Meneses, 1999). The serotonin level in the brain increases after administration of an SSRI and this affects various post- and presynaptic 5-HT receptors. Decreasing the serotonin level by tryptophan depletion also affects the 5-HT receptors although differently. Increasing or decreasing the serotonin level in the brain does not have to have opposite effects on memory. One study investigated the effect of serotonin increase by means of 3,4-methylenedioxymetamphetamine (MDMA) administration in human volunteers on verbal memory and showed a memory impairing effect on immediate and delayed verbal recall (Kuypers \& Ramaekers, 2005). In contrast to tryptophan depletion and SSRI administration which are involved in memory consolidation, MDMA administration apparently also impairs immediate recall, possibly mediated through 5- $\mathrm{HT}_{1 \mathrm{~A}}$ receptor or 5- $\mathrm{HT}_{2 \mathrm{~A}}$ receptor agonism (Kuypers \& Ramaekers, 2005). This also supports the assumption of that the association between serotonin and memory is complex involving specific serotonin receptor subtypes.

In the present study, it was expected that mirtazapine would impair memory performance and, essentially, a significant overall impairment was found. In other words, mirtazapine impaired immediate verbal recall during 16 days. Analyzing for each test day separately, it was not possible to assign this effect to an initial dose, dose increase or steady state effect. Impairment of psychomotor and cognitive performance is common after single and repeated doses of alpha2-antagonists such as mirtazapine (Ramaekers et al., 1998; Wingen et al., 2005). Detrimental effects of mirtazapine on cognition are generally believed to result from the drugs' high affinity for the $\mathrm{H} 1$ receptor. Blockade of the brain $\mathrm{H} 1$ receptors is well known to cause drowsiness and sedation and result in acute psychomotor and cognitive impairment (Hindmarch \& Shamsi, 1999; O'Hanlon \& Ramaekers, 1995; Theunissen et al., 2006). Importantly, although it is said that mirtazapine is a well tolerated antidepressant that improves sleep in depressed patients (Nutt, 2002), it can still reduce daytime alertness the day after the initial dose (Radhakishun et al., 2000). Notably, the results of the present study are not completely generalizable to older medication users because of the relative limited age range of the study. In addition, different memory tasks or paradigms might show more pronounced treatment effects.

In summary, escitalopram 10 and $20 \mathrm{mg}$ had no effect on a visual verbal memory task that measured immediate and delayed recall and recognition. A role of serotonin in memory could not be further established. Mirtazapine $30 \mathrm{mg}$ and $45 \mathrm{mg}$ 
revealed a slightly impairing overall effect on immediate recall, probably due to its sedative effects caused by $\mathrm{H} 1$ receptor blockade.

\section{Acknowledgments}

This study was sponsored by H. Lundbeck A/S, Denmark. 


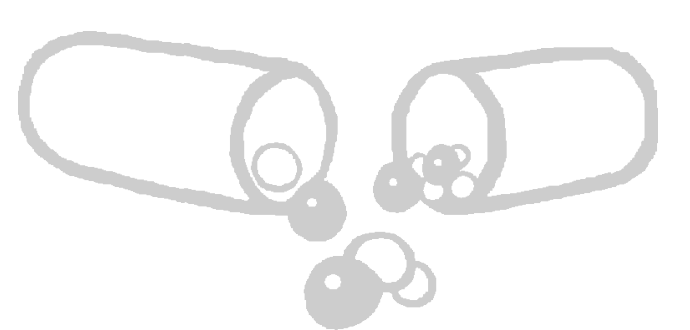

\title{
Chapter 6 \\ Selective verbal and spatial memory impairment after $5-\mathrm{HT}_{1 \mathrm{~A}}$ and $5-\mathrm{HT}_{2 \mathrm{~A}}$ receptor blockade in healthy volunteers pre-treated with an SSRI
}

\begin{abstract}
Introduction: Serotonergic neurotransmission has been implicated in memory impairment. It is unclear however if memory performance is mediated through general serotonin availability, through specific 5-HT receptors or both. The aim of the present study was to assess the contribution of serotonin reuptake inhibition and specific blockade of $5-\mathrm{HT}_{1 \mathrm{~A}}$ and $5-\mathrm{HT}_{2 \mathrm{~A}}$ receptors to memory impairment. Methods: The study was conducted according to a randomized, double-blind, placebo controlled, 4-way crossover design including 16 healthy volunteers. The treatment consisted of oral administration of escitalopram $20 \mathrm{mg}+$ placebo, escitalopram $20 \mathrm{mg}$ + ketanserin $50 \mathrm{mg}$, escitalopram $20 \mathrm{mg}$ + pindolol $10 \mathrm{mg}$ and placebo on 4 separate days with a wash-out period of minimum 7 days. Different memory tasks were performed including verbal memory, spatial working memory and reversal learning. Results: Escitalopram showed an impairing effect on immediate verbal recall which nearly reached statistical significance. No effects of escitalopram were found on other types of memory. In combination with pindolol, immediate verbal recall was significantly impaired. Escitalopram in combination with ketanserin impaired spatial working memory significantly. No effects were found on reversal learning. Conclusion: Selective impairment of immediate verbal recall after a $5-\mathrm{HT}_{1 \mathrm{~A}}$ partial agonist and selective impairment of spatial working memory performance after $5-\mathrm{HT}_{2 \mathrm{~A}}$ receptor antagonist, both in combination with a selective serotonergic reuptake inhibitor (escitalopram), suggests that $5-\mathrm{HT}_{1 \mathrm{~A}}$ and $5-\mathrm{HT}_{2 \mathrm{~A}}$ receptors are distinctly involved in verbal and spatial memory.
\end{abstract}

Wingen M, Kuypers KPC, Ramaekers JG (2006): Selective verbal and spatial memory impairment after $5-\mathrm{HT}_{1 \mathrm{~A}}$ and $5-\mathrm{HT}_{2 \mathrm{~A}}$ receptor blockade in healthy volunteers pretreated with an SSRI. Journal of Psychopharmacology DOI:10.1177/0269881106072506. 


\section{Introduction}

In humans, the association between memory function and serotonin has mostly been studied in tryptophan depletion studies and in serotonergic challenge studies. Acute trypthophan depletion studies have consistently shown to reduce memory consolidation (Harrison et al., 2004; Riedel, Klaassen et al., 1999; Schmitt et al., 2000) which implicates that lowered serotonin levels are associated with memory impairment. Challenge studies with acute and chronic administration of selective serotonin reuptake inhibitors (SSRIs) that enhance serotonin levels (Anderson et al., 2005) have provided inconsistent results by showing negative (Riedel et al., 2005), neutral (Riedel et al., 2005; Wilkinson et al., 2003; Wingen et al., 2006) as well as positive (Harmer et al., 2002) effects on memory consolidation. Likewise, other serotonergic drugs such as MDMA have been shown to impair verbal and spatial memory performance during intoxication when serotonin levels are high (Kuypers \& Ramaekers, 2005; Kuypers \& Ramaekers, 2006) but also during abstinence when serotonin levels are low (Curran \& Travill, 1997; Parrott et al., 1998). Consequently, it has been proposed that memory function may be mediated through some alternative mechanism such as serotonergic receptor activation rather than through synaptic serotonin availability alone (Kuypers \& Ramaekers, 2005).

Studies in animals as well as humans have provided strong evidence for an involvement of $5-\mathrm{HT}_{1 \mathrm{~A}}$ and $5-\mathrm{HT}_{2}$ receptors in different aspects of memory. The 5$\mathrm{HT}_{1 \mathrm{~A}}$ receptor can be found pre- and post-synaptically and is highly concentrated in the hippocampus and in the raphe nucleus. In animals, $5-\mathrm{HT}_{1 \mathrm{~A}}$-agonist or $5-\mathrm{HT}_{1 \mathrm{~A}}$ antagonists have been shown to respectively produce memory improvement or impairment (Buhot et al., 2000). In humans, administration of a 5-HT $\mathrm{HT}_{1 \mathrm{~A}}$ agonist impaired immediate and delayed verbal recall presumably by stimulating the postsynaptic 5- $\mathrm{HT}_{1 \mathrm{~A}}$ receptor in the hippocampus (Yasuno et al., 2003). The 5- $\mathrm{HT}_{1 \mathrm{~A}}$ agonist ipsapirone also impaired immediate verbal memory in healthy volunteers but improved immediate verbal memory in depressed patients (Riedel, Klaassen, Griez et al., 2002). Post-synaptic 5- $\mathrm{HT}_{2 \mathrm{~A}}$ receptors can be found in high levels in cerebral cortical areas and at intermediate levels in the hippocampus, nucleus accumbens and the hypothalamus (Dwivedi \& Pandey, 1998). A role for $5-\mathrm{HT}_{2}$ receptors in memory seems present, although the exact mechanism is not clear (Meneses, 2002). In healthy humans the $5-\mathrm{HT}_{2 \mathrm{~A}}$ receptor was shown to be involved in memory by the fact that a polymorphism of the gene encoding the 5- $\mathrm{HT}_{2 \mathrm{~A}}$ receptor affected delayed memory recall negatively. Carriers of heterozygous variant, which indicates a blunted receptor 
response, had a poorer episodic memory performance compared to carriers of the common variant (de Quervain et al., 2003).

The aim of this study was to further assess the contribution of general serotonin levels and serotonergic receptor function to memory performance. General elevation of synaptic serotonin levels was achieved by pretreating healthy volunteers with an SSRI, i.e. escitalopram. Escitalopram is the most selective SSRI available in dosages of 10 and $20 \mathrm{mg}$ (Aronson \& Delgado, 2004). Additional blockade of 5- $\mathrm{HT}_{1 \mathrm{~A}}$ and $5-\mathrm{HT}_{2 \mathrm{~A}}$ receptors, was achieved by subsequent administration of pindolol and ketanserin respectively. Pindolol is a very partial $5-\mathrm{HT}_{1 \mathrm{~A}}$ agonist that blocks $5-\mathrm{HT}_{1 \mathrm{~A}}$ receptors preferentially presynaptically (Rabiner et al., 2004; Rabiner et al., 2000). The indirect inhibiting effect of the SSRI on serotonin via the 5- $\mathrm{HT}_{1 \mathrm{~A}}$ autoreceptor is attenuated by pindolol which leads to increased serotonin levels (Rasmussen et al., 2004). Consequently, it was expected that pindolol would augment the effect of escitalopram on memory. SSRIs in combination with pindolol have also been used in preclinical studies to augment or accelerate the antidepressant effect of SSRIs (Blier et al., 1998). To obtain a $37 \%$ occupancy of the $5-\mathrm{HT}_{1 \mathrm{~A}}$ autoreceptor a $10 \mathrm{mg}$ single dose of pindolol was necessary in healthy volunteers (Rabiner et al., 2001). Ketanserin has previously shown to be a valuable drug for studying the role of $5-\mathrm{HT}_{2 \mathrm{~A}}$ receptors in human performance (Liechti et al., 2000; Vollenweider et al., 1998). It was suggested that the agonistic effect of MDMA on the dopaminergic system through $5-\mathrm{HT}_{2}$ stimulation was blocked by ketanserin $50 \mathrm{mg}$ (Liechti et al., 2000). In the present study, it was expected that blocking the $5-\mathrm{HT}_{2 \mathrm{~A}}$ receptor by ketanserin would decrease dopamine release (Bortolozzi et al., 2005; Lucas \& Spampinato, 2000) which in theory would also augment the effects of the SSRI on memory.

\section{Methods}

\section{Subjects}

Sixteen healthy volunteers $(8 \propto, 8 \hat{)})$ mean age (se) $25,9(1,73)$ were recruited. All participants underwent a screening procedure consisting of a telephone interview, health questionnaire, 12-lead electrocardiogram, laboratory test (haematology and blood chemistry, urinalysis, drug-and pregnancy screening) and a routine medical examination. Volunteers were included when they were 21 to 45 years of age, healthy (i.e. absence of all exclusion criteria), had a normal static binocular acuity (corrected or uncorrected), a body mass index between 19 and 30, and were willing to sign an informed consent. Excluded were those volunteers who 
suffered from, or had a history of cardiac, hepatic, renal, pulmonary, neurological, gastrointestinal, haematological or psychiatric illness. Other exclusion criteria were excessive drinking (>20 glasses of alcohol containing beverages a week), pregnancy or lactation, menstrual disorder, use of medication other than oral contraceptives, smoking tobacco or use of illicit drugs, and any sensory or motor deficits which could reasonably be expected to affect test performance. Those volunteers who had a firstdegree relative with a psychiatric disorder or a history of a psychiatric disorder were also excluded. The study was approved by the standing medical ethics committee of Maastricht University and the Maastricht Academic Hospital's Board of Directors. It was carried out in accordance with the World Medical Association's Declaration of Helsinki (Edinburgh, 2000). Written informed consent was obtained from each volunteer prior to participation to the study.

\section{Design and treatment}

The study was conducted according to a double-blind, placebo controlled, 4-way crossover design. Complete balancing of the treatment orders yielded sixteen treatment orders randomly assigned to 16 healthy human volunteers. Balancing the treatments to prevent order effects, results in every treatment being administered for a given period in the same number. Treatments consisted of escitalopram $20 \mathrm{mg}+$ placebo, escitalopram $20 \mathrm{mg}$ + ketanserin $50 \mathrm{mg}$, escitalopram $20 \mathrm{mg}+$ pindolol 10 $\mathrm{mg}$ and placebo + placebo administered at 4 different test days separated by a washout period of at least 7 days (mean (se), range: $8,5(0,48), 7-23)$ to prevent carryover effects. Escitalopram oral dose has a Cmax around 4 hours and a half-life of 27-32 hours (Aronson \& Delgado, 2004). Ketanserin and pindolol also oral dosages have a Cmax of 2 hours and 1 hour and a half-life of 3-4 hours and 13-18 hours respectively (Aellig et al., 1981; Hedner et al., 1983).

\section{Testing procedure}

Participants were trained prior to their first treatment condition on cognitive assessments to minimize learning effects. Participants arrived at 9.00 a.m. at the test laboratory; received a standard breakfast and completed a sleep quality questionnaire. They received the first treatment capsule, containing either escitalopram or placebo at 9.30 a.m. Participants were then seated for the next $4 \mathrm{hrs}$ in a secluded waiting room in order to wait for escitalopram to reach Cmax. At 12 hours participants received a standard lunch, followed by the second treatment 
capsule at 12.30 p.m. containing pindolol, ketanserin or placebo. Mood assessment and cognitive assessments were conducted at 13.30 p.m., i.e. at $1 \mathrm{hr}$ after the second drug treatment. Participants were not allowed to consume alcohol 24 hours prior to testing and caffeine-containing beverages 4 hours prior to testing.

\section{Cognitive assessments}

The Visual Verbal Learning task (VVLT)

The VVLT, a modified version of the Rey Auditory Verbal Learning Test (Rey, 1964) consisted of a list of 30 monosyllabic words in Dutch, which were presented in three trials on a computer screen (Riedel, Klaassen et al., 1999). Immediately after each presentation (immediate recall) and 20 minutes after the last presentation (delayed recall) participants were asked to verbally reproduce as many of the presented words as possible. Twenty-five minutes after the last presentation a delayed recognition test was administered. A series of thirty words were shown (fifteen new and fifteen old words from the original wordlist) and the participant was asked to press 'yes' for 'familiar' and 'no' for 'new', as quickly as possible. According to the theory of signal detection (Pollack et al., 1964), the proportion of correct recognized words (cr) and the proportion of falsely recognized (fr) constitute the nonparametric sensitivity measure: $\mathrm{A}^{\prime}=1-1 / 4(\mathrm{fr} / \mathrm{cr}+(1-\mathrm{cr}) /(1-\mathrm{fr})) \cdot \mathrm{A}^{\prime}$ is in fact the proportion of correctly recognized words corrected for the participant's response tendency. $A^{\prime}$ was arcsin transformed before statistical analyses.

The outcome measurements were the number of correct words recalled during the three immediate recall trials as a measure of short term memory, the number of correct words produced on delayed free recall as a measure of retrieval from long term memory, $\mathrm{A}^{\prime}$ as a measure of storage in long term memory, and the mean reaction times of correctly recognized words as a measure of speed of long term memory.

\section{Spatial Memory Task (SMT)}

The spatial memory task assessed short-term memory for spatial information (Vermeeren et al., 1995). The spatial memory task was based on spatial localization. The participant was briefly shown a fixation point in the centre of the computer display. Shortly thereafter, a target appeared at a random location for $500 \mathrm{msec}$. The participants' task was to memorize the position of the target and, using a trackball, to relocate the cursor as accurately as possible over that position. The cursor appeared 
either immediately upon target offset or after a delay of 2 or $4 \mathrm{sec}$. The participant depressed a button to indicate that the cursor was at the recalled position of the target. The test consisted of 75 trials, divided equally among 3 response delays. The sequence of delays was random. Regression lines were calculated to describe localization error (in $\mathrm{mm}$ ) as a function of response delay. The slope reflects memory decay, and the intercept the participant's localization error.

\section{Probabilistic Reversal Learning task (RVL)}

In the reversal-learning task (Cools et al., 2002) two abstract stimuli, composed of two bars of different colour, were randomly presented to the left or right side of a computer screen (Evers, Tillie et al., 2005). One stimulus was advantageous (S+) and was usually (70\%) associated with large reward (addition of 80 - 250 points) and occasionally (30\%) with a small punishment (subtraction of $10-60$ points), based on a pseudo-random sequence. The other stimulus was disadvantageous (S-) and was usually (60\%) associated with a large punishment (250 - 600 points) and occasionally (40\%) with a small reward (30 - 60 points). Volunteers had to determine which stimulus was advantageous based on the feedback; the number of points won or lost. Once this was learned, i.e. the advantageous stimulus was chosen four times out of five responses, the stimulus-reward contingencies were reversed ( $\mathrm{S}+$ became $\mathrm{S}_{-}$, and S- became S+). The participant's task was to keep track of the most profitable stimulus and to collect as many points as possible. The task duration was 10 minutes or 121 trials. Outcome measurements were total number of reversals, total number of perseverations in proportion to the reversals, total number of points collected, overall mean reaction time and mean reaction time of the first correct response after a reversal switch.

\section{Subjective measurement}

Participants rated feelings of depression, tension, fatigue, vigour and anger by means of the Profile Of Mood States (McNair et al., 1992).

\section{$\underline{\text { Statistical analyses }}$}

Data were analyzed by means of a GLM repeated measures analysis (SPSS 11.5) with Treatment (4 levels) as main factor. In case of a significant overall effect of Treatment, follow-up contrast tests were used to test for differences between drugs and placebo 
and between combination treatments and escitalopram. The alpha criterion significance level was set at $\mathrm{p}=0.05$.

\section{$\underline{\text { Results }}$}

Missing data

All 16 subjects completed the study, there were no missing values. Drugs were tolerated well by all participants during the test days. For spontaneously reported side effects see Table 1.

Table 1. Spontaneously reported side effects

\begin{tabular}{l||c|c|c|c}
\hline & Escitalopram & $\begin{array}{c}\text { Esitalopram } \\
+ \text { ketanserin }\end{array}$ & $\begin{array}{c}\text { Escitalopram } \\
+ \text { pindolol }\end{array}$ & Placebo \\
\hline \hline Drowsiness during test day & $6 \%$ & $25 \%$ & $12,5 \%$ & - \\
\hline Nausea during test day & $6 \%$ & $6 \%$ & $6 \%$ & - \\
\hline Drowsiness started the next evening & - & - & $12,5 \%$ & - \\
\hline Nausea startedthe next evening & - & $12,5 \%$ & - & - \\
\hline
\end{tabular}

\section{Cognitive assessments}

A summary of mean (se) performance scores on all cognitive and subjective measures in each treatment condition is given in Table 2. Mean differences (confidence intervals), estimates of effect sizes and observed power are given in Table 3.

The Visual Verbal Learning task (VVLT)

Overall, Treatment significantly affected total immediate recall $\left(\mathrm{F}_{3,45}=3.96, \mathrm{p}<.05\right)$. Drug-placebo contrasts showed a slight impairing effect of escitalopram that approached statistical significance $\left(\mathrm{F}_{1,15}=3.54, \mathrm{p}=.08\right)$. Furthermore, escitalopram + pindolol impaired total immediate recall significantly $\left(\mathrm{F}_{1,15}=8.19, \mathrm{p}<.05\right)$. Drug-drug comparison showed that total immediate recall after escitalopram + pindolol was worse compared to escitalopram alone $\left(\mathrm{F}_{1,15}=5.525, \mathrm{p}<.05\right)$. No interaction effects were found between Treatment and learning trials. No effects were found on delayed recall and delayed recognition. Mean (se) immediate recall scores in every treatment condition for the separate learning trials are shown in Figure 1. 
Table 2. Mean (se) outcome variables of memory tasks and subjective measurement

\begin{tabular}{|c|c|c|c|c|c|c|}
\hline \multirow{3}{*}{ Treatment } & \multicolumn{6}{|c|}{ Mean (standard error of mean) } \\
\hline & \multirow{2}{*}{ Escitalopram } & \multirow{2}{*}{$\begin{array}{l}\text { Escitalopram } \\
+ \text { ketanserin } \\
\end{array}$} & \multirow{2}{*}{$\begin{array}{c}\text { Escitalopram } \\
+ \text { pindolol }\end{array}$} & \multirow{2}{*}{ Placebo } & \multicolumn{2}{|c|}{ Overall } \\
\hline & & & & & $F, d f=3,45$ & $p$ \\
\hline \multicolumn{7}{|l|}{ VVLT } \\
\hline Total immediate recall & $41,9(2,28)$ & $42,1(2,39)$ & $37,8(2,82)^{\mathrm{A}, \mathrm{C}}$ & $44,1(1,23)$ & 3.96 & $<.05$ \\
\hline Delayed recall & $13,1(1,23)$ & $13,2(1,35)$ & $12,4(1,57)$ & $14,2(1,44)$ & 0.88 & ns \\
\hline \multicolumn{7}{|l|}{ Delayed recognition } \\
\hline \# Correct recalled words & $13,6(0,29)$ & $13,6(0,34)$ & $13,2(0,38)$ & $12,8(0,48)$ & 1.53 & ns \\
\hline Mean RT (msec) & $787(37,67)$ & $787(40,44)$ & $774(31,77)$ & $787(29,66)$ & 0.12 & ns \\
\hline $\mathrm{A}^{\prime}$ & $0,94(0,01)$ & $0,96(0,01)$ & $0,95(0,01)$ & $0,96(0,02)$ & 0.95 & ns \\
\hline \multicolumn{7}{|l|}{ SMT } \\
\hline Localization error delay 0 (mm) & $8,4(0,44)$ & $11,1(1,05)^{\mathrm{B}, \mathrm{C}}$ & $8,1(0,59)$ & $7,8(0,41)$ & 5.99 & $<.01$ \\
\hline Localization error delay 2 (mm) & $11,3(0,77)$ & $14,3(1,26)^{\mathrm{A}, \mathrm{C}}$ & $11,5(0,79)$ & $11,1(0,84)$ & 3.03 & $<.05$ \\
\hline Localization error delay 4 (mm) & $13,7(1,06)$ & $16,3(1,19)^{\mathrm{A}}$ & $13,8(0,79)$ & $12,6(0,94)$ & 2.78 & $=.05$ \\
\hline Mean RT delay 0 (msec) & $2240(181,59)$ & $2403(269,55)$ & $2126(146,24)$ & $2026(110,55)$ & 1.85 & ns \\
\hline Mean RT delay 2 (msec) & $2128(151,84)$ & $2243(219,55)$ & $1930(123,11)$ & $2055(151,86)$ & 2.53 & ns \\
\hline Mean RT delay 4 (msec) & $2289(198,37)$ & $2302(267,06)$ & $2013(151,64)$ & $2048(143,44)$ & 1.72 & ns \\
\hline Intercept & $8,5(0,44)$ & $11,3(1,08)^{\mathrm{A}, \mathrm{C}}$ & $8,4(0,55)$ & $8,2(0,44)$ & 5.18 & $<.05$ \\
\hline Slope & $1,3(0,23)$ & $1,3(0,15)$ & $1,4(0,10)$ & $1,2(0,18)$ & 0.44 & ns \\
\hline \multicolumn{7}{|l|}{ RVL } \\
\hline \# reversals & $14,8(0,90)$ & $13,9(0,70)$ & $14,0(1,00)$ & $14,4(1,05)$ & 0.37 & ns \\
\hline \# perseverations & $3,86(0,88)$ & $5,06(2,30)$ & $1,81(0,53)$ & $1,07(0,33)$ & 1.80 & ns \\
\hline \# points & $9082(782,95)$ & $9505(935,08)$ & $10201(826,31)$ & $9248(785,13)$ & 1.16 & ns \\
\hline Mean RT (msec) & $690(68,8)$ & $612(34,50)$ & $596(51,57)$ & $603(46,97)$ & 2.79 & ns \\
\hline Mean RT after reversal (msec) & $679(68,31)$ & $621(46,4)$ & $583(49,30)$ & $589(42,45)$ & 1.83 & ns \\
\hline \multicolumn{7}{|l|}{ POMS } \\
\hline Depression & $20,8(3,64)^{\mathrm{A}}$ & $20,78(3,20)$ & $25,8(4,57)$ & $17,0(2,62)$ & 2.94 & $<.05$ \\
\hline Anger & $20,9(3,08)^{\mathrm{A}}$ & $16,7(2,33)$ & $21,6(3,14)$ & $16,4(2,41)$ & 3.74 & $<.05$ \\
\hline Fear & $35,6(4,19)^{\mathrm{B}}$ & $31,0(4,62)$ & $36,1(6,32)^{\mathrm{A}}$ & $20,9(2,65)$ & 3.93 & $<.05$ \\
\hline Vigor & $30,4(4,76)^{\mathrm{B}}$ & $29,6(4,82)^{\mathrm{B}}$ & $35,9(5,89)^{\mathrm{A}}$ & $17,7(2,75)$ & 4.88 & $<.01$ \\
\hline Tension & $17,9(2,36)$ & $18,6(2,51)$ & $22,6(3,66)$ & $15,3(2,37)$ & 2.32 & ns \\
\hline
\end{tabular}

$\mathrm{A}=\mathrm{p}<.05$ as indicated by separate drug-placebo contrasts

$B=p<.01$ as indicated by separate drug-placebo contrasts

$\mathrm{C}=\mathrm{p}<.05$ as indicated by separate drug contrasts compared to escitalopram

$\mathrm{ns}=$ non significant 
Table 3. Mean differences (confidence intervals), estimates of effects sizes and observed power of significant drug-placebo contrasts

\begin{tabular}{|c|c|c|c|}
\hline & \multicolumn{3}{|c|}{ Drug placebo contrast } \\
\hline & \multicolumn{3}{|c|}{ Escitalopram } \\
\hline & Mean differnce $(95 \% \mathrm{CI})$ & $\begin{array}{l}\text { Estimates of } \\
\text { effect size }\end{array}$ & Observed Power \\
\hline $\begin{array}{l}\text { VVLT } \\
\text { Total immediate recall }\end{array}$ & ns & 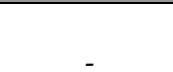 & _- \\
\hline SMT & & & \\
\hline Localization error delay $0(\mathrm{~mm})$ & ns & - & - \\
\hline Localization error delay $2(\mathrm{~mm})$ & ns & - & - \\
\hline Localization error delay $4(\mathrm{~mm})$ & ns & - & - \\
\hline Intercept & ns & - & - \\
\hline \multicolumn{4}{|l|}{ POMS } \\
\hline Depression & $-6,36(-12,09--0.69)$ & 0,272 & 0,60 \\
\hline Anger & $-4,40(-8,27--0,54)$ & 0,282 & 0,62 \\
\hline Fear & $-11,62(-18,50--4,75)$ & 0,464 & 0,92 \\
\hline \multirow[t]{2}{*}{ Vigor } & $12,66(3,95-21,37)$ & 0,390 & 0,83 \\
\hline & \multicolumn{3}{|c|}{ Escitalopram + ketanserin } \\
\hline VVLT & & & \\
\hline Total immediate recall & ns & - & - \\
\hline SMT & & & \\
\hline Localization error delay $0(\mathrm{~mm})$ & $3,26(1,06-5,46)$ & 0,399 & 0,84 \\
\hline Localization error delay $2(\mathrm{~mm})$ & $3,20(0,17-6,24)$ & 0,252 & 0,56 \\
\hline Localization error delay $4(\mathrm{~mm})$ & $3,74(0,60-6,89)$ & 0,300 & 0,66 \\
\hline Intercept & $3,07(0,67-5,48)$ & 0,330 & 0,72 \\
\hline \multicolumn{4}{|l|}{ POMS } \\
\hline Depression & ns & - & - \\
\hline Anger & ns & - & - \\
\hline Fear & ns & - & - \\
\hline \multirow[t]{2}{*}{ Vigor } & 11,88 & 0,237 & 0,52 \\
\hline & \multicolumn{3}{|c|}{ Escitalopram + pindolol } \\
\hline \multicolumn{4}{|l|}{ VVLT } \\
\hline Total immediate recall & $-6,31(-11,01--1,61)$ & 0,353 & 0,76 \\
\hline \multicolumn{4}{|l|}{ SMT } \\
\hline Localization error delay $0(\mathrm{~mm})$ & ns & - & - \\
\hline Localization error delay $2(\mathrm{~mm})$ & ns & - & - \\
\hline Localization error delay $4(\mathrm{~mm})$ & ns & - & - \\
\hline Intercept & ns & - & - \\
\hline \multicolumn{4}{|l|}{ POMS } \\
\hline Depression & ns & - & - \\
\hline Anger & ns & - & - \\
\hline Fear & $-15,17(-27,22--3,12)$ & 0,324 & 0,71 \\
\hline Vigor & $18,20(6,13-30,27)$ & 0,408 & 0,85 \\
\hline
\end{tabular}

ns= non significant 
Figure 1 Mean (se) recall of words after 3 learning trials as performance measurement in the Visual Verbal Learning Task

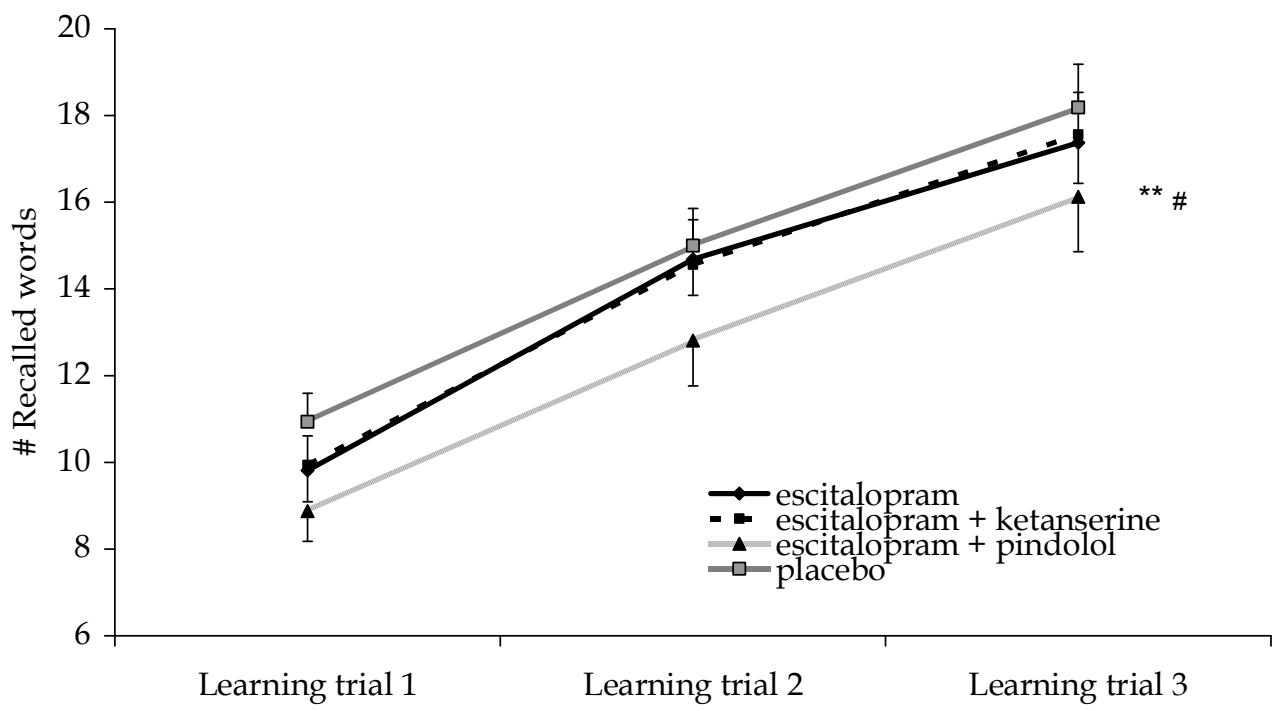

$* *=p<0.05$ escitalopram + pindolol compared to placebo as indicated by separate drug-placebo contrasts

$\#=$ p $<0.05$ escitalopram + pindolol compared to escitalopram as indicated by a separate drug contrast

\section{Spatial Memory Task (SMT)}

A significant overall effect of Treatment was found on localisation error in each response delay condition: i.e. after a response delay of 0 sec (delay 0$)\left(F_{3,45}=5.99\right.$, $\mathrm{p}<.01), 2 \mathrm{sec}$ (delay 2) $\left(\mathrm{F}_{3,45}=3.03, \mathrm{p}<.05\right)$ and $4 \mathrm{sec}$ (delay 4$)\left(\mathrm{F}_{3,45}=2.78, \mathrm{p}=.05\right)$. Drugplacebo contrasts showed an impairing effect of escitalopram + ketanserin in each response delay condition; i.e. delay $0\left(\mathrm{~F}_{1,15}=9.94, \mathrm{p}<.01\right)$, delay $2\left(\mathrm{~F}_{1,15}=5.06, \mathrm{p}<.05\right)$ and delay $4\left(\mathrm{~F}_{1,15}=6.44, \mathrm{p}<.05\right)$. Drug-drug comparison showed that localisation error was increased on delay $0\left(\mathrm{~F}_{1,15}=6.08, \mathrm{p}<.05\right)$ and delay $2\left(\mathrm{~F}_{1,15}=4.98, \mathrm{p}<.05\right)$ compared to escitalopram. These results indicate that escitalopram + ketanserin increased localization error independent of response delay. The latter notion was also evinced by the fact that combined treatment of escitalopram + ketanserin affected the intercept $\left(F_{1,15}=7.40, p<.05\right)$ compared to placebo and compared to escitalopram $\left(F_{1,15}=5.957\right.$, $\mathrm{p}<.05)$, but not the slope of the regression equation determining localization error as a 
function of response delay. Reaction time was not affected by Treatment. Means (se) localization error in every treatment condition is shown in Figure 2.

Figure 2 Mean (se) localization error of the Spatial Memory Task as a function of response delay

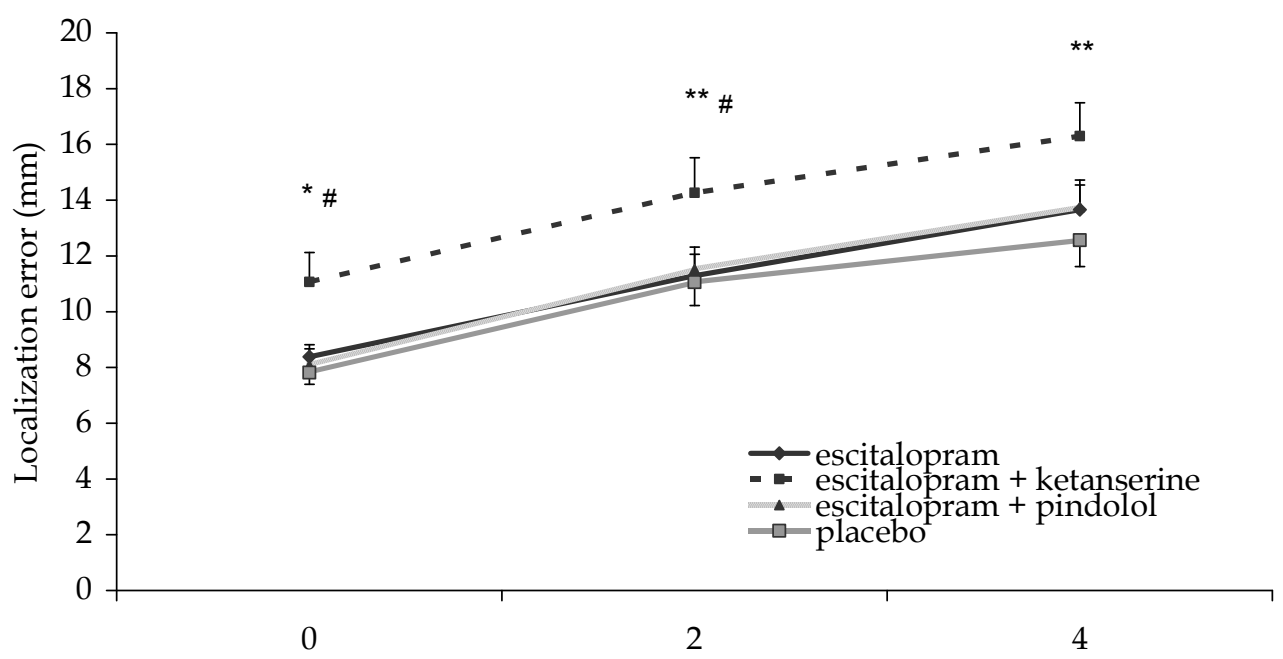

Response delay (sec)

$*=p<0.01$ escitalopram + ketanserin compared to placebo as indicated by separate drug-placebo contrasts

$* *=p<0.05$ escitalopram + ketanserin compared to placebo as indicated by separate drugplacebo contrasts

$\#=p<0.05$ escitalopram + ketanserin compared to escitalopram as indicated by a separate drug contrast

Probabilistic Reversal Learning task (RVL)

No statistically significant Treatment effects were established on the RVL.

\section{Subjective measurement}

Overall effects of Treatment were found on feelings of depression $\left(\mathrm{F}_{3,45}=2.94, \mathrm{p}<.05\right)$, anger $\left(\mathrm{F}_{3,45}=3.74, \mathrm{p}<.05\right)$, fatigue $\left(\mathrm{F}_{3,45}=3.93, \mathrm{p}<.05\right)$, and vigour $\left(\mathrm{F}_{3,45}=4.88, \mathrm{p}<.01\right)$. Drugplacebo contrasts showed significantly increased feelings of depression after escitalopram $\left(\mathrm{F}_{1,15}=5.59, \mathrm{p}<.05\right)$ and nearly significantly increased feelings of depression after escitalopram + pindolol $\left(\mathrm{F}_{1,15}=4.04, \mathrm{p}=.06\right)$. Furthermore, escitalopram 
significantly increased feelings of anger $\left(\mathrm{F}_{1,15}=5.89, \mathrm{p}<.05\right)$ and escitalopram + pindolol slightly increased feelings of anger although not significantly different from placebo $\left(F_{1,15}=3.75, p=.07\right)$. Feelings of fatigue were significantly increased by escitalopram $\left(\mathrm{F}_{1,15}=12.96, \mathrm{p}<.01\right)$ and escitalopram + pindolol $\left(\mathrm{F}_{1,15}=7.20, \mathrm{p}<.05\right)$ and nearly significantly increased after escitalopram + ketanserin $\left(\mathrm{F}_{1,15}=4.22, \mathrm{p}=.06\right)$. Feelings of vigour were significantly decreased by escitalopram $\left(F_{1,15}=9.60, p<.01\right)$, escitalopram + ketanserin $\left(\mathrm{F}_{1,15}=4.66, \mathrm{p}<.05\right)$ and escitalopram + pindolol $\left(\mathrm{F}_{1,15}=10.34, \mathrm{p}<.01\right)$ compared to placebo. No Significant correlations were found between the different scales of the POMS and the cognitive measurements.

\section{Discussion}

Escitalopram did not affect spatial memory and reversal learning, but tended to impair immediate recall in a verbal learning task. Escitalopram furthermore produced feelings of depression, anger and fear and decreased feelings of vigour. Additional treatment with pindolol augmented the effects of escitalopram and caused significant impairment of immediate verbal recall, elevated feelings of anger and fear and deceased vigour. Additional treatment with ketanserin resulted in significant impairment of spatial memory performance and less vigour. These findings suggest that $5-\mathrm{HT}_{1 \mathrm{~A}}$ and $5-\mathrm{HT}_{2 \mathrm{~A}}$ receptors are distinctly involved in verbal memory and spatial working memory processing respectively.

Verbal memory impairment approached statistical significance after treatment with escitalopram but additional treatment with pindolol significantly augmented this deficit compared to placebo as well as escitalopram alone. This combined effect of escitalopram and pindolol was very selective and confined to verbal memory performance only. The mechanism of escitalopram and pindolol on serotonin release may be twofold. Firstly, pindolol binds at presynaptic $5-\mathrm{HT}_{1 \mathrm{~A}}$ autoreceptors where it disinhibits serotonin release and contributes to the general increase of serotonin caused by escitalopram (Rasmussen et al., 2004). Secondly, pindolol binds at the postsynaptic $5-\mathrm{HT}_{1 \mathrm{~A}}$ receptor where it blocks signal transmission. The former might be the main mechanism underlying verbal memory impairment as pindolol was shown to preferentially bind at the presynaptic autoreceptors (Rabiner et al., 2004; Rabiner et al., 2000). Prior studies on the association between memory function and brain levels of serotonin have shown that both elevated and reduced levels of serotonin have been associated with impairment of immediate recall and delayed recall measures in verbal memory tasks (Harrison et al., 2004; Riedel, Klaassen et al., 1999; Schmitt et al., 2000). 
It has been suggested that the association between serotonergic activity and cognition can be described in terms of an inverted U-curve (Meeter et al., 2006; Schmitt et al., 2006). That is, both understimulation as well as overstimulation of the serotonergic system resulting from either serotonin depletion or serotonin suppletion may imbalance the cortical network responsible for memory function and lead to impairment of certain aspects of memory such as learning and consolidation. In the present study, pindolol would lead to a scenario of overstimulation by increasing the overall availability of serotonin in the synaptic cleft.

Spatial working memory performance decreased after combined treatment with escitalopram and ketanserin but not after treatment with escitalopram alone. As before, the effect of this combined treatment was very selective and confined to spatial working memory. The mechanisms underlying spatial working memory impairment clearly must be related to blockade of post-synaptic $5-\mathrm{HT}_{2 \mathrm{~A}}$ receptors by kentanserin because memory impairment increased after combined escitalopram/ketanserin treatment compared to escitalopram alone. The exact role of 5- $\mathrm{HT}_{2}$ receptors in memory function however is rather unclear (Meneses, 2002). Indications are present for $5-\mathrm{HT}_{2}$ receptors to modulate dopaminergic activity. Moreover, $5-\mathrm{HT}_{2 \mathrm{~A}}$ receptors and $5-\mathrm{HT}_{2 \mathrm{c}}$ receptors exert opposite effects on dopamine release (Esposito, 2006). Evidence has been provided that $5-\mathrm{HT}_{2 \mathrm{c}}$ receptors inhibit dopamine pathways (Di Matteo et al., 2001; Lucas \& Spampinato, 2000) and that 5- $\mathrm{HT}_{2 \mathrm{~A}}$ receptors enhance dopamine release (Bortolozzi et al., 2005; Lucas \& Spampinato, 2000). Dopamine enhancement occurs in the Ventral Tegmental Area (VTA) which gives rise to the mesocortical dopamine system (Bortolozzi et al., 2005) and the mesocortical dopamine system is regarded as an important modulator of cognition such as spatial working memory (Ellis et al., 2005; Robbins, 2000). For example, dopaminergic stimulation by bromocriptine, a dopamine agonist has been demonstrated to improve spatial working memory (Luciana et al., 1998) whereas decrements in dopamine levels after tyrosine depletion has been shown to impair spatial working memory (Harmer et al., 2001). The implication is that blockade of the $5-\mathrm{HT}_{2 \mathrm{~A}}$ receptor by ketanserin in the present study may have decreased dopamine release in the mesocortical pathway which in turn may have resulted in impairment of spatial working memory performance.

It is noteworthy that the impairing effect of combined escitalopram and ketanserin treatment on spatial working memory performance occurred independent or did not interact with variations in response delay. In other words, combined 
treatment affected the intercept but not the slope of the regression lines describing localization error as a function of response delay. One could argue that spatial memory impairments were caused by a general perceptual deficit following combined treatment of escitalopram and ketanserin. However, the fact that impairment of spatial memory was selective and specific to escitalopram/ketanserin and did not appear in other tasks in which visual perception was also required, refutes this argument. Furthermore, it is not expected that the effects caused by the differential serotonergic manipulations were due to side effects caused by the drug treatments. Participants generally reported loss of vigour and increase in anger, fatigue and drowsiness to similar degrees in all drug conditions which cannot account for the specificity and selectivity of memory impairments that were observed in the present study under different drug conditions. In addition, no correlations were found between mood and cognition.

No significant effects of the serotonergic manipulations were found on reversal learning. The former seems in line with a number of previous studies that failed to show any behavioural effects of acute tryptophan depletion on reversal learning (Evers, Tillie et al., 2005; Evers, Cools et al., 2005; Gallagher et al., 2003; Hughes, Gallagher et al., 2003; Murphy et al., 2002). Apart from the present study, no other work on the effects of serotonin suppletion on reversal learning is available. The overall conclusion thus seems to be that task performance in reversal learning tasks is not strongly dependent on serotonergic mechanisms.

In the present study, pindolol, a 5- $\mathrm{HT}_{1 \mathrm{~A}}$ partial agonist, impaired immediate verbal memory and ketanserin, a $5-\mathrm{HT}_{2 \mathrm{~A}}$ receptor antagonist, impaired spatial memory, both in combination with the SSRI escitalopram. These results suggest that 5$\mathrm{HT}_{1 \mathrm{~A}}$ receptors and $5-\mathrm{HT}_{2}$ receptor are distinctly involved in verbal and spatial memory function.

\section{Acknowledgments}

The authors are thankful to Silke Conen and medical doctor C.J. van Leeuwen, for their assistance in data collection. 


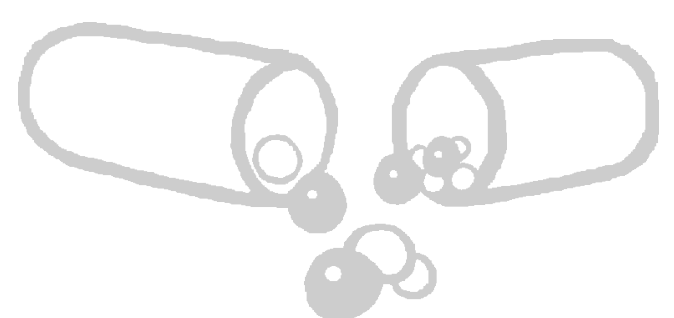

\title{
Chapter 7 \\ The role of $5-\mathrm{HT}_{1 \mathrm{~A}}$ and $5-\mathrm{HT}_{2 \mathrm{~A}}$ receptors in attention and motor control: a mechanistic study in healthy volunteers
}

\begin{abstract}
Rationale: various studies have demonstrated a modulating role for serotonin in attention. Selective serotonin inhibitors have repeatedly been shown to impair performance in sustained attention tasks.

Objectives: to assess the contribution of serotonin reuptake inhibition and specific blockade of the presynaptic $5-\mathrm{HT}_{1 \mathrm{~A}}$ receptor and the $5-\mathrm{HT}_{2} \mathrm{~A}$ receptor to deficits in attention. Methods: the study was conducted according to a randomized, double-blind, placebo controlled, 4-way crossover design including 16 healthy volunteers. Treatments consisted of oral administration of the SSRI escitalopram $20 \mathrm{mg}+$ placebo, escitalopram $20 \mathrm{mg}+$ ketanserin (5- $\mathrm{HT}_{2 \mathrm{~A}}$ antagonist) $50 \mathrm{mg}$, escitalopram $20 \mathrm{mg}+$ pindolol $\left(5-\mathrm{HT}_{1 \mathrm{~A}}\right.$ antagonist) $10 \mathrm{mg}$ and placebo + placebo on 4 separate days. A range of performance tasks were conducted to assess the subjects' attention and motor functions. Results: escitalopram administered alone impaired tracking performance in a divided attention task. The combination of escitalopram and pindolol as well as escitalopram and ketanserin impaired divided attention as compared to placebo. In addition, escitalopram and ketanserin impared sustained attention. Divided attention impairment observed after combined treatments did not significantly differ from impairments after escitalopram alone. Sustained attention impairment observed after combined escitalopram and ketanserin significantly differed from escitalopram alone. Conclusions: 5-HT $1 \mathrm{~A}$ receptor blockade hardly affected SSRI effects on attention. Additional 5-HT $2 \mathrm{~A}$ receptor blockade however produced impairments of sustained attention.
\end{abstract}

Wingen, M., Kuypers, K. P. C., \& Ramaekers, J. G. (2007). The role of 5-HT1A and 5$\mathrm{HT}_{2 \mathrm{~A}}$ receptors in attention and motor control: a mechanistic study in healthy volunteers. Psychopharmacology, 190, 391-400. 


\section{Introduction}

Suppletion of serotonin (5-HT) levels has been associated with a reduction in sustained attention i.e. the capability to stay alert for a period of time, often called vigilance. Enhancement of serotonin levels after acute and subchronic administration of a range of selective serotonin reuptake inhibitors (SSRIs), i.e. fluoxetine, paroxetine, venlafaxine and citalopram, has been shown to reduce vigilance performance as assessed by various studies in healthy volunteers using the Mackworth Clock paradigm (O'Hanlon et al., 1998; Ramaekers et al., 1995; Riedel et al., 2005; Schmitt, Ramaekers et al., 2002). The Mackworth Clock Task has been extensively used in studies on human vigilance performance. The task last 45 minutes during which subjects must sustain attention on the task, while waiting for an unpredictable event (Mackworth, 1950).

The role of serotonin in vigilance performance has not been fully determined, but may be indirectly related to dopaminergic neurotransmission. Stimulation of dopamine transmission by amphetamine and methylphenidate has been shown to improve vigilance performance (Koelega, 1993) and dopamine has been associated with tonic internally-driven arousal and readiness to respond (Pribram \& McGuinness, 1975; Robbins, 1997). Serotonin exerts an inhibitory influence on dopaminergic activity (Soubrie, 1986; Spoont, 1992) which in turn could lead to vigilance impairment. The role of dopamine in SSRI induced vigilance impairment was previously demonstrated in two studies assessing the effect of sertralin on vigilance (Riedel et al., 2005; Schmitt, Ramaekers et al., 2002). Both studies showed that sertralin does not reduce vigilance, probably because the drug not only inhibits serotonin transporters but dopamine transporters as well. This additional dopaminergic stimulation is believed to attenuate the vigilance impairment following indirect serotonin stimulation by sertralin (Schmitt, Ramaekers et al., 2002).

The aim of the study was to further examine the association between serotonin and attention and the contributing roles of specific serotonin receptors. Therefore, the present study examined the role of $5-\mathrm{HT}_{1 \mathrm{~A}}$ and $5-\mathrm{HT}_{2 \mathrm{~A}}$ receptors in serotonin related impairment of attention in subjects that were pre-treated with an SSRI. Pre-treatment by the SSRI escitalopram, was expected to stimulate serotonergic functionby elevating serotonin levels in the synaptic cleft by indirect activation of $5-\mathrm{HT}_{1}$ and $5-\mathrm{HT}_{2}$ receptors. Subsequently, blockade of $5-\mathrm{HT}_{1 \mathrm{~A}}$ and $5-\mathrm{HT}_{2 \mathrm{~A}}$ receptors was achieved by administration of pindolol and ketanserin respectively. Evidence exists that $5-\mathrm{HT}_{2}$ receptors modulate dopaminergic activity (Esposito, 2006). It was expected that 
blocking the postsynaptic $5-\mathrm{HT}_{2 \mathrm{~A}}$ receptor by ketanserin may decrease dopamine release (Bortolozzi et al., 2005; Lucas \& Spampinato, 2000). A decrease in dopamine may lead to less activation of the mesocortical dopamine projections to the prefrontal cortex (Robbins, 1997) which in turn may augment the impairing effect of the SSRI on sustained attention. It was expected that blocking the $5-\mathrm{HT}_{1 \mathrm{~A}}$ receptor by pindolol would augment the effect of escitalopram on sustained attention. Pindolol is a very partial $5-\mathrm{HT}_{1 \mathrm{~A}}$ agonist that blocks $5-\mathrm{HT}_{1 \mathrm{~A}}$ receptors preferentially presynaptically (Rabiner et al., 2004; Rabiner et al., 2000). The indirect inhibiting effect or feedback mechanism of the SSRI on serotonin via the presynaptic 5- $\mathrm{HT}_{1 \mathrm{~A}}$ autoreceptor is attenuated by pindolol which leads to increased serotonin levels (Rasmussen et al., 2004). In addition to vigilance performance, other tasks measuring divided attention, selective attention, motor control and motor impulse control were included in the present study to determine the effects of the abovementioned serotonergic manipulations on these cognitive functions.

\section{Methods}

\section{Subjects}

Sixteen healthy volunteers $(8 \uparrow, 8 \jmath)$ mean age (se) $25,9(1,73)$ were recruited. All participants underwent a screening procedure consisting of a telephone interview, health questionnaire, 12-lead electrocardiogram, laboratory testing (haematology and blood chemistry, urinalysis, drug-and pregnancy screening) and a routine medical examination. Volunteers were included when they were 21 to 45 years of age, healthy (i.e. absence of all exclusion criteria), had a normal static binocular acuity (corrected or uncorrected), a body mass index between 19 and 30, and were willing to sign an informed consent. Excluded were those volunteers who suffered from, or had a history of cardiac, hepatic, renal, pulmonary, neurological, gastrointestinal, haematological or psychiatric illness. Other exclusion criteria were excessive drinking (>20 glasses of alcohol containing beverages a week), pregnancy or lactation, menstrual disorder, use of medication other than oral contraceptives, smoking tobacco or use of illicit drugs, and any sensory or motor deficits which could reasonably be expected to affect test performance. Those volunteers who had a first-degree relative with a psychiatric disorder or a history of a psychiatric disorder were also excluded. Eventually, 3 out of 8 women used oral contraceptives. The study was approved by the standing medical ethics committee of Maastricht University and the Maastricht Academic Hospital's Board of Directors. It was carried out in accordance with the 
World Medical Association's Declaration of Helsinki (Edinburgh, 2000). Written informed consent was obtained from each volunteer prior to participation to the study.

\section{Design and treatment}

The study was conducted according to a double-blind, placebo controlled, 4-way crossover design. Complete balancing of the treatments yielded sixteen treatment orders that were randomly assigned to 16 healthy human volunteers. Treatments consisted of escitalopram $20 \mathrm{mg}+$ placebo, escitalopram $20 \mathrm{mg}+$ ketanserin $50 \mathrm{mg}$, escitalopram $20 \mathrm{mg}$ + pindolol $10 \mathrm{mg}$ and placebo + placebo administered at 4 different test days separated by a wash-out period of at least 7 days. Escitalopram, ketanserin and pindolol oral dosages have a Cmax of 4 hours, 2 hours and 1 hour and a half-life of 27-32 hours, 3-4 hours and 13-18 hours respectively (Aellig et al., 1981; Hedner et al., 1983). An oral dose of $10 \mathrm{mg}$ of pindolol was needed in healthy volunteers to achieve a $37 \%$ occupancy of the $5-\mathrm{HT}_{1 \mathrm{~A}}$ autoreceptor (Rabiner et al., 2001). A $50 \mathrm{mg}$ single dose of Ketanserin was used based on a previous study (Liechti et al., 2000).

\section{Testing procedure}

Participants underwent a training session of the performance test in order to minimize learning effects. On test days, participants arrived at 9.00 a.m. at the test laboratory; received a standard breakfast and completed a sleep quality questionnaire. They received the first treatment capsule, containing either escitalopram or placebo at 9.30 a.m. Participants were then seated for the next $4 \mathrm{hrs}$ in a secluded waiting room in order to wait for escitalopram to reach Cmax. At 12 hours participants received a standard lunch, followed by the second treatment capsule at 12.30 p.m. containing pindolol, ketanserin or placebo. Mood assessment and cognitive assessments were conducted at 13.30 p.m., i.e. at $1 \mathrm{hr}$ after the second drug treatment. Participants were not allowed to consume alcohol 24 hours prior to testing and caffeine-containing beverages 4 hours prior to the start of the test day.

\section{Performance tasks measuring attention}

\section{Sustained attention - Mackworth Clock Test}

The Mackworth Clock Test measures vigilance or sustained attention (Mackworth, 1950). Participants were seated in front of a computer screen displaying a circular 
arrangement of 60 gray dots. The dots were briefly illuminated in clockwise rotation at a rate of one per 500 milliseconds, moving 6 degrees from dot to dot. Volunteers were instructed that occasionally there would be a skip of illumination of one of the dots (12 degrees move of the dots). At this signal volunteers were required to press a button as fast as possible. A response within 3 seconds of the signal was registered as a correct detection. A total of 30 signals were presented with 10 per 15 minute period and intervals of 8 seconds to 7.2 minutes. Outcome measures were total correct detections (hits) and corresponding reaction times.

\section{Selective attention - Left-right task}

The left-right task or choice reaction time task is a parametric version of the wellknown colour-word response conflict task (Stroop, 1935). The words 'Left' and 'Right' were displayed either at the left or the right side of a computer screen. Response instructions were to respond quickly (by pressing a corresponding button) to the location of the word irrespective of its meaning. The outcome variables were correct detections for compatible and incompatible words with the corresponding reaction times. A compatible word stands for the word 'left' or 'right' presented at the corresponding side of the computer screen. An incompatible word means that the word 'left' was presented at the right side of the computer screen or the word 'right' was presented at the left side of the computer screen.

\section{Divided attention - Divided Attention Task}

Divided Attention Task (DAT) (Moskowitz, 1973)measures the ability to divide attention between two tasks performed simultaneously. Participants performed the same tracking task as described below (CTT) but at a constant level of difficulty set at $50 \%$ of his/her maximum capacity. Tracking error is measured as the difference in millimetre between the position of the cursor and the midpoint of the scale. While tracking, the participant monitored 24 peripheral displays upon which single digits change asynchronously at 5 seconds intervals. The occurrence of the digit " 2 " was a signal for the participant to remove the foot from a pedal as rapidly as possible. Signals occurred twice at every location, in random order, at intervals of 5 to 25 seconds. Average absolute tracking error, mean reaction time, hits and control losses were taken as outcome variables. 
Performance tasks measuring motor control

Motor control - Critical Tracking Task

The Critical Tracking Task (CTT) (Jex et al., 1966)measures the participant's ability to control a displayed error signal in a first-order compensatory tracking task. Error was displayed as an increasing horizontal deviation of a cursor from the midpoint on a horizontal, linear scale. Compensatory joystick movements nulled the error by returning the cursor to the midpoint. The frequency, at which the participant lost control, was the critical frequency or lambda ${ }_{\mathrm{c}}\left(\lambda_{\mathrm{c}}\right)$ in rad/s. The test included 5 trials of which the lowest and the highest score were discarded the average of the remaining scores was taken as the final score.

\section{Motor inhibition - Stop signal task}

This task requires participants to make quick key responses to visually presented go signals and to inhibit any response when a visual stop signal occurs (Ramaekers \& Kuypers, 2006). The go signals were four $1.5 \mathrm{~cm}$ letters (ABCD) presented one at a time in the centre of a computer screen. Participants were required to respond to each letter as quickly as possible by pressing on one of two response buttons. One button was pressed to indicate that " $\mathrm{A}$ " or " $\mathrm{C}$ " appeared and the other to indicate " $\mathrm{B}$ " or " $\mathrm{D}$ ". Letters were displayed for $500 \mathrm{~ms}$ and the computer screen was blank for $1.5 \mathrm{~s}$ interstimulus interval before the next letter was displayed. This provides a period of 2 sec in which the participant can respond to a letter.

A single test consisted of 176 trials in which each of the 4 letter stimuli were presented equally often. A stop signal occurred in 48 trials during a test. The stop signal consisted of visual cue, i.e. "*", that appeared in one of the four corners of the screen. Participants were required to withhold any response in case a stop-signal was presented. Stop signal were presented 12 times at each of the four delays after the onset of a letter: 50, 150, 250 and $350 \mathrm{~ms}$. Trials always begin with a $500 \mathrm{~ms}$ preparation interval in which a fixation point appeared on the centre of the screen. The task lasted about 10 minutes. Outcome measures were correct responses to the go signals, successfully inhibited responses per delay (correct inhibitions), mean reaction time of the go signals and an estimated time required to inhibit responses (stop signal reaction time). The stop signal reaction time was calculated on the probability of inhibiting responses to stop signals and the distribution of reaction times to go-signals after excluding outliers (Logan, 1994). 


\section{Subjective measurements}

Each test day subjects filled out the Groninger Sleep Quality Scale (Mulder-Hajonides van der Meulen, 1981) to assess sleep quality during the preceding night. The total score consisted of 14 yes/no questions to score the number of sleep complaints (ranging from good sleep (score 0) to worst possible sleep (score 14)). In addition, specific questions about time needed to fall asleep, number of awakenings during the night and sleep duration in hours were included. Bond and Lader Questionnaire was assessed using different scales for Alertness, Contentedness and Calmness (Bond \& Lader, 1974).

\section{Statistical analyses}

Data were analyzed by means of a GLM repeated measures analysis (SPSS 11.5) with Treatment (4 levels) as the main factor. Only when a significant overall effect of Treatment was found on a particular performance measure (alpha level was set at $\mathrm{p}=0.05)$, follow-up contrast analyses were conducted to test for differences between treatments and placebo and between combined treatments and escitalopram. For follow-up contrast analyses, alpha level was set at $\mathrm{p}=0.01$ to correct for multiple (i.e. 5) comparisons.

\section{$\underline{\text { Results }}$}

\section{Missing data}

A total of 16 subjects completed the study. Due to technical failure data of one subject on the Mackworth clock task in the escitalopram + ketanserin condition was not recorded. These incomplete data were treated as missing values and replaced by the mean score of the remaining 15 subjects in the escitalopram + ketanserin condition.

\section{Cognitive assessments}

Mean (se) of the cognitive assessments are shown in Table 1. Confidence intervals, estimates of effect size and observed power are presented in Table 2.

\section{Cognitive outcome parameters measuring attention}

\section{Sustained attention - Mackworth Clock Test}

Overall, Treatment significantly affected correct detections $\left(\mathrm{F}_{3,45}=9.06, \mathrm{p}<.001\right)$. In addition there was a Time on task effect for correct detections $\left(\mathrm{F}_{3,45}=12.57, \mathrm{p}<.001\right)$, which indicates a vigilance decrement during the 45 minutes task performance, but no 
Treatment by Time on task interaction effect. Drug-placebo contrasts showed that escitalopram + ketanserin $\left(\mathrm{F}_{1,15}=37.59, \mathrm{p}<.001\right)$ reduced the total number of correct detections significantly, as compared to placebo. Drug-drug comparisons demonstrated that escitalopram + ketanserin significantly impaired correct detections $\left(\mathrm{F}_{1,15}=8.065, \mathrm{p}=.01\right)$ compared to escitalopram alone. Escitalopram alone or escitalopram + pindolol did not affect the outcome measurements of the Mackworth Clock Test. No Treatment effects or Time on task effects were found on the reaction time. Mean (se) number of correct detections in every treatment condition as a function of time on task is shown in Figure 1.

Table 1. Mean (se) outcome variables of cognitive tasks

\begin{tabular}{|c|c|c|c|c|c|c|}
\hline \multirow{3}{*}{$\begin{array}{l}\text { Treatment } \\
\text { Outcome measures } \\
\end{array}$} & \multicolumn{6}{|c|}{ Mean (standard error of mean) } \\
\hline & \multirow{2}{*}{ Escitalopram } & \multirow{2}{*}{$\begin{array}{l}\text { Escitalopram } \\
+ \text { ketanserin } \\
\end{array}$} & \multirow{2}{*}{$\begin{array}{c}\text { Escitalopram } \\
+ \text { pindolol }\end{array}$} & \multirow{2}{*}{ Placebo } & \multicolumn{2}{|c|}{ Overall } \\
\hline & & & & & $\mathrm{F}, \mathrm{df}=3,45$ & $\mathbf{P}$ \\
\hline \multicolumn{7}{|l|}{ Mackworth Clock Task } \\
\hline Correct detections (hits) & $21,4(1,48)$ & $15,2(1,56)^{\mathrm{B}, \mathrm{D}}$ & $19,8(1,50)$ & $22,7(1,04)$ & 9.07 & $<.001$ \\
\hline mean RT (msec) & $797(63,6)$ & $905(87,9)$ & $788(65,6)$ & $770(62,0)$ & 2.47 & ns \\
\hline \multicolumn{7}{|l|}{ Choice Reaction TimeTask } \\
\hline Correct compatible & $15,8(0,10)$ & $15,9(0,06)$ & $15,8(0,14)$ & $15,9(0,09)$ & 0.65 & ns \\
\hline Mean RT compatible (msec) & $574(19,6)$ & $623(29,2)$ & $590(23,9)$ & $571(24,7)$ & 2.15 & ns \\
\hline Correct incompatible & $15,5(0,16)$ & $15,5(0,16)$ & $15,4(0,22)$ & $15,4(0,16)$ & 0.09 & ns \\
\hline Mean RT incompatible (msec) & $586(20,1)$ & $618(24,7)$ & $601(22,0)$ & $574(15,9)$ & 2.76 & .053 \\
\hline \multicolumn{7}{|l|}{ Divided Attention Task } \\
\hline Average tracking error (mm) & $19,0(1,52)^{\mathrm{B}}$ & $20,2(1,62)^{B}$ & $19,2(1,87)^{\mathrm{B}}$ & $17,1(1,47)$ & 5.67 & .002 \\
\hline Mean RT (msec) & $1831(66,0)$ & $1955(84,4)$ & $1872(96,7)$ & $1759(75,7)$ & 2.45 & ns \\
\hline Hits & $45,7(0,70)$ & $44,6(0,89)$ & $45,2(1,28)$ & $45,6(0,73)$ & 1.50 & ns \\
\hline Control losses & $5,2(1,73)$ & $10,4(4,13)$ & $6,2(2,20)$ & $7,2(3,27)$ & 1.07 & ns \\
\hline \multicolumn{7}{|l|}{ Critical Tracking Task } \\
\hline $\operatorname{lambda}_{c}\left(\lambda_{c}\right)$ & $3,7(0,20)$ & $3,8(0,19)$ & $3,9(0,18)$ & $3,8(0,18)$ & 0.69 & ns \\
\hline \multicolumn{7}{|l|}{ Stop Signal Task } \\
\hline Correct detections & $116,8(3,28)$ & $115,7(3,41)$ & $118,4(1,76)$ & $118,7(2,85)$ & 0.59 & ns \\
\hline Correct inhibitions 350 & $3,6(0,83)$ & $4,5(0,91)$ & $4,0(1,06)$ & $3,8(0,98)$ & 0.88 & ns \\
\hline 250 & $7,7(0,95)$ & $7,1(1,03)$ & $7,1(1,08)$ & $7,3(1,01)$ & 0.48 & ns \\
\hline 150 & $9,8(0,66)$ & $9,2(0,83)$ & $9,4(0,90)$ & $9,1(0,78)$ & 0.54 & ns \\
\hline 50 & $10,8(0,50)$ & $10,2(0,65)$ & $10,5(0,75)$ & $10,6(0,63)$ & 1.10 & ns \\
\hline Mean RT hits (msec) & $555(29,0)$ & $604(37,0)$ & $578(45,3)$ & $567(40,9)$ & 2.64 & ns \\
\hline Stop Signal RT (msec) & $273(12,0)$ & $307(19,4)$ & $256(13,7)$ & $285(11,3)$ & 3.89 & .028 \\
\hline
\end{tabular}

$\mathrm{B}=\mathrm{p}<.01$ as indicated by separate drug-placebo contrasts

$\mathrm{D}=\mathrm{p}=.01$ as indicated by separate drug-drug comparisons compared to escitalopram ns= not significant 
Table 2. Mean differences (confidence intervals), estimates of effects sizes and observed power of significant drug-placebo contrasts

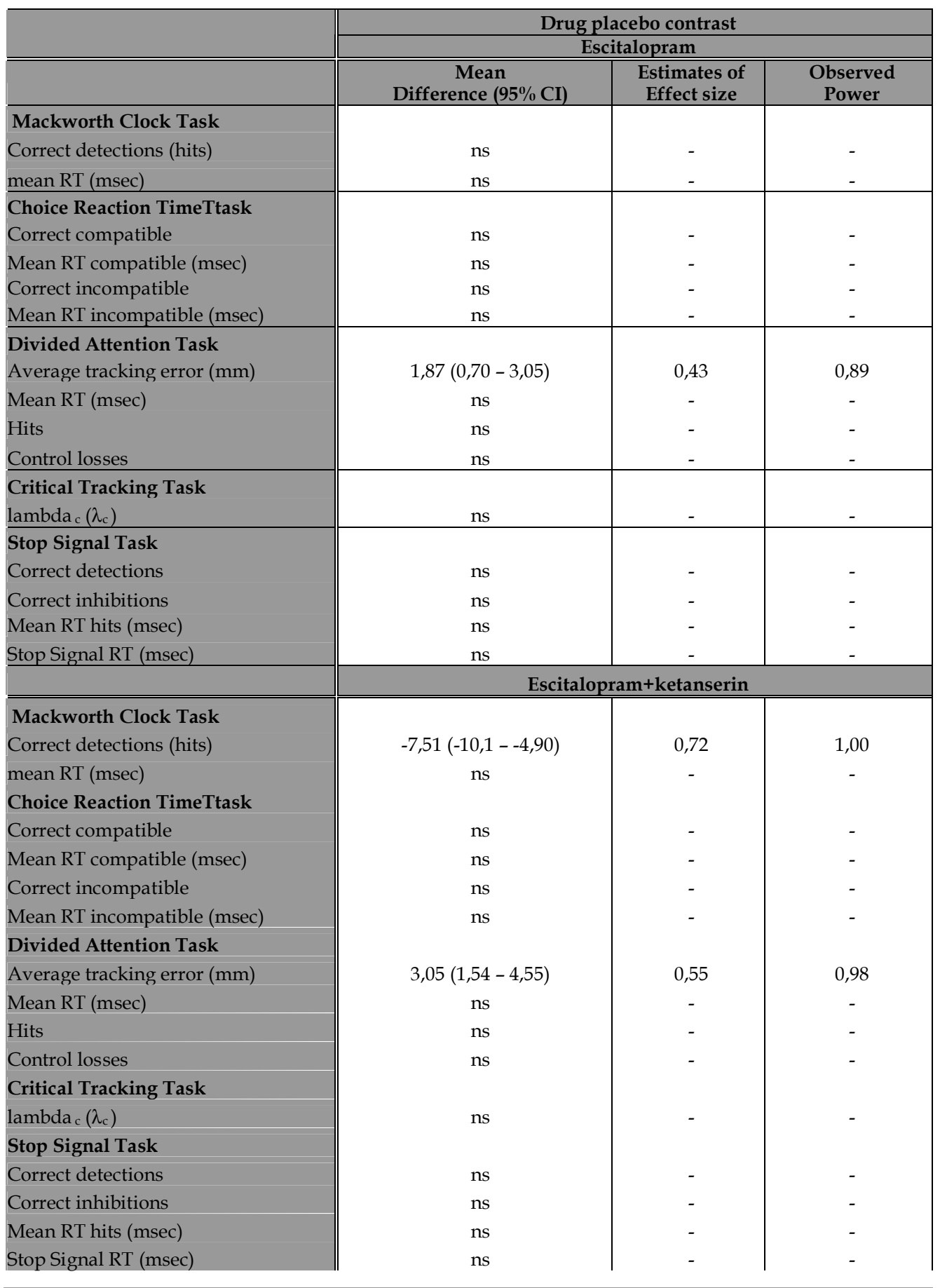


(Table 2 continued)

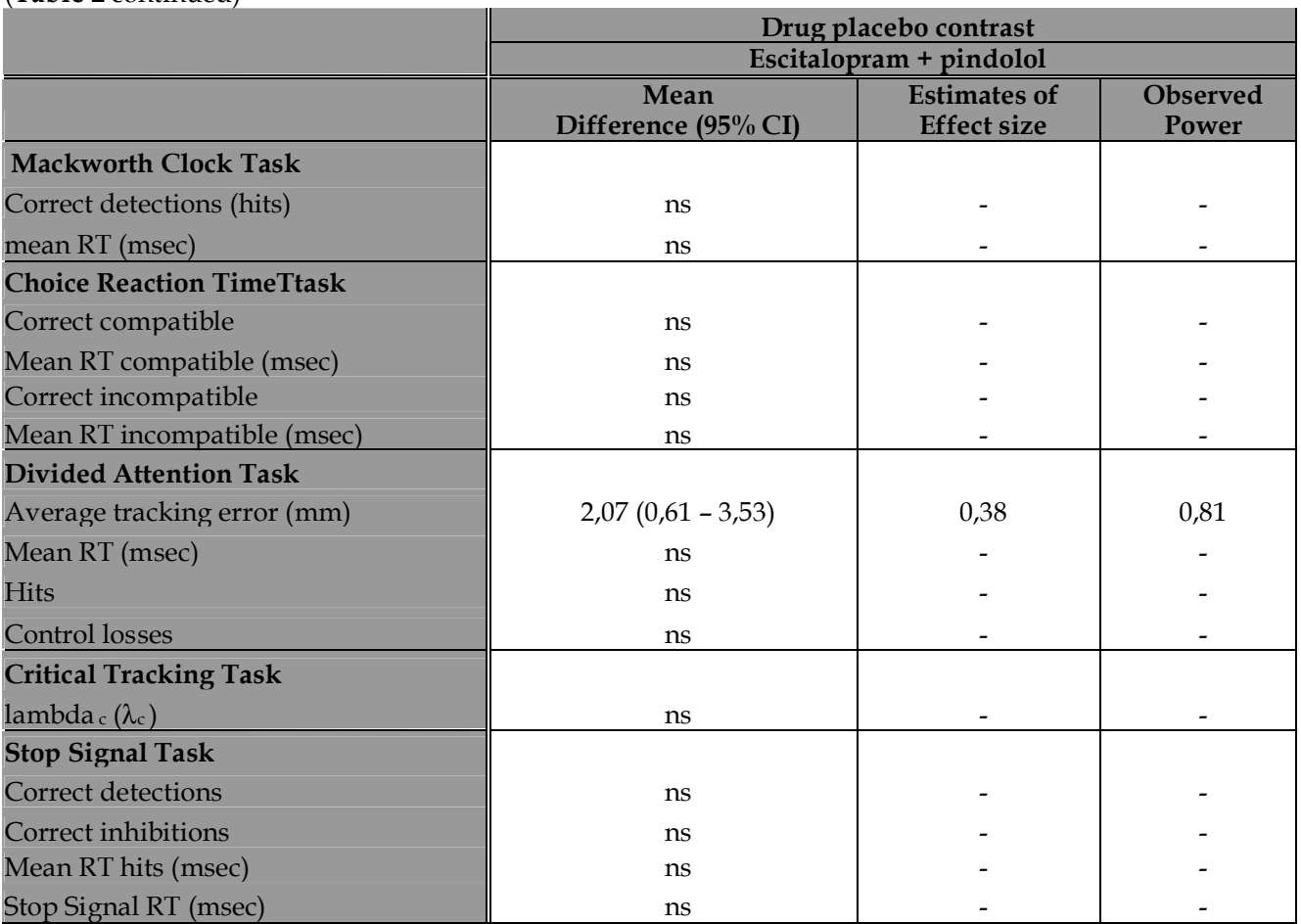

ns= non significant

\section{Selective attention - Left-right task}

A significant overall effect of Treatment was found on mean reaction time in incompatible trials $\left(\mathrm{F}_{3,45}=2.76, \mathrm{p}=05\right)$. Drug-placebo contrasts and drug-drug contrasts did not reveal any differences. No effect was found on other outcome measurements of the selective attention task.

\section{Divided attention - Divided Attention Task}

Treatment $\left(\mathrm{F}_{3,45}=5.67, \mathrm{p}<.01\right)$ significantly affected tracking performance in the divided attention task. Escitalopram $\left(\mathrm{F}_{1,15}=11.52, \mathrm{p}<.01\right)$, escitalopram + pindolol $\left(\mathrm{F}_{1,15}=9.13\right.$, $\mathrm{p}<.01)$ and escitalopram + ketanserin $\left(\mathrm{F}_{1,15}=18.64, \mathrm{p}<.001\right)$ all increased tracking error as compared placebo. Mean (se) of tracking error in every treatment condition is shown in Figure 2. Drug-drug contrasts did not reveal any differences. No treatments effects were found on other outcome measurements of the divided attention task. 
Figure 1 Mean (se) number of hits as performance measurement in the Mackworth Clock Task

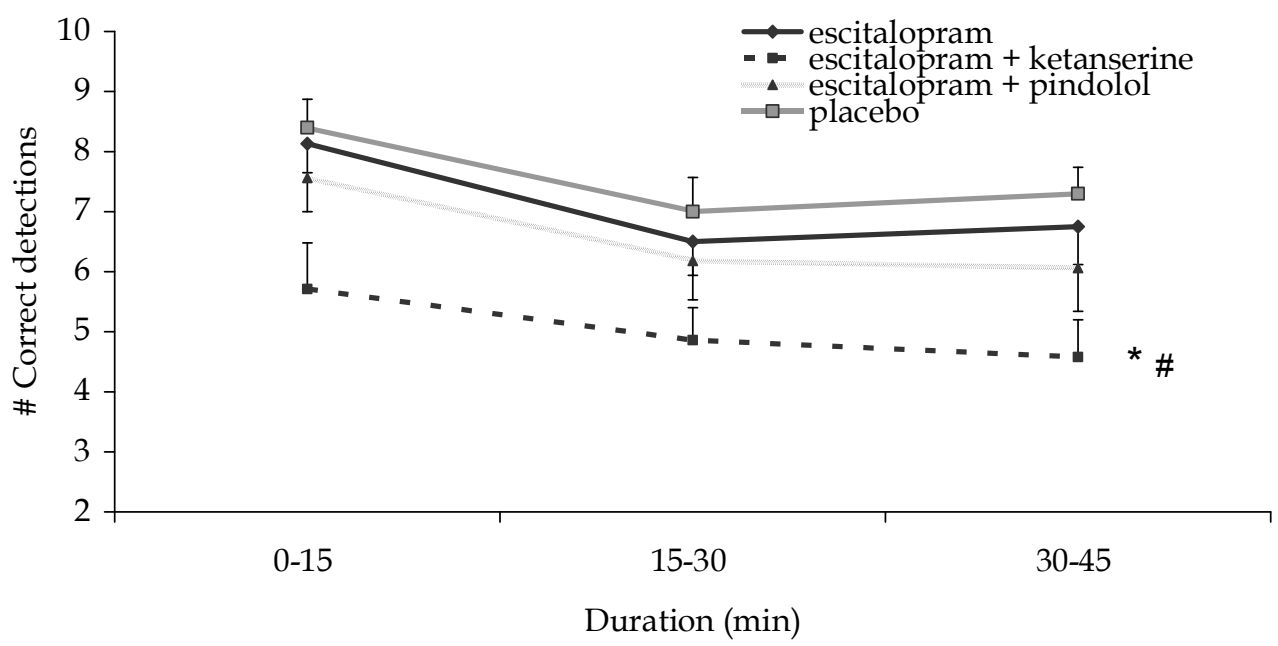

$*=p<.01$ escitalopram + ketanserin compared to placebo as indicated by separate drug-placebo contrasts

$\#=p=.01$ as indicated by separate drug-drug comparisons escitalopram + ketanserin compared to escitalopram

Figure 2 Mean (se) tracking error of the Divided Attention Task

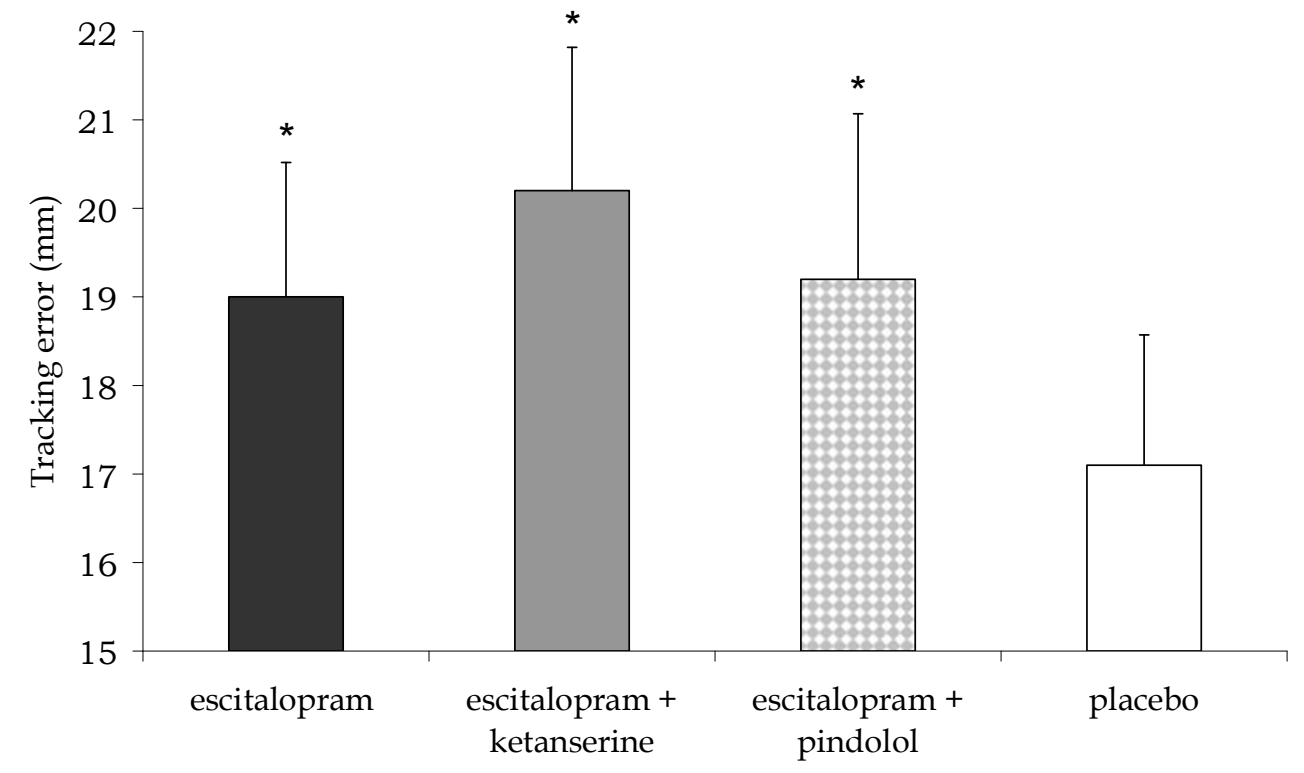

$*=p<.01$ compared to placebo as indicated by separate drug-placebo contrasts 


\section{Cognitive outcome parameters measuring motor control}

Motor control - Critical Tracking Task

No Treatment effects were found on the mean lambda score.

Motor inhibition - Stop signal task

A significant effect of Treatment was found on stop signal reaction time $\left(\mathrm{F}_{3,45}=3.89\right.$, $\mathrm{p}<.05)$. Drug-placebo and drug-drug contrasts did not reveal any significant differences. No Treatment effects were found on correct detections, reaction times or correct inhibitions per delay.

\section{Subjective measurements}

Subjective evaluations of sleep quality and mood are shown in Table 3.

Treatments did not affect the outcome measures of the Groninger Sleep Quality Scale and the Contentedness and Calmness ratings but did affect the Alertness ratings $\left(\mathrm{F}_{3,45}=3,65, \mathrm{p}<.05\right)$. Drug-placebo comparisons did not reveal any differences. Subsequent analysis showed no significant correlations between the alertness ratings and the significant attention performance measurements.

Table 3. Mean (se) outcome variables of subjective measurements

\begin{tabular}{|c|c|c|c|c|c|c|}
\hline \multirow{3}{*}{$\begin{array}{l}\text { Treatment } \\
\text { Outcome measures }\end{array}$} & \multicolumn{6}{|c|}{ Mean (standard error of mean) } \\
\hline & \multirow{2}{*}{ Escitalopram } & \multirow{2}{*}{$\begin{array}{l}\text { Escitalopram } \\
+ \text { ketanserin }\end{array}$} & \multirow{2}{*}{$\begin{array}{c}\begin{array}{c}\text { Escitalopram } \\
+ \text { pindolol }\end{array} \\
\end{array}$} & \multirow{2}{*}{ Placebo } & \multicolumn{2}{|l|}{ Overall } \\
\hline & & & & & $\mathrm{F}, \mathrm{df}=3,45$ & $\mathbf{P}$ \\
\hline \multicolumn{7}{|l|}{ Groninger Sleep Quality Scale } \\
\hline Number of sleep complaints & $2,6(0,71)$ & $1,4(0,39)$ & $2,2(0,69)$ & $0,88(0,42)$ & 2.42 & ns \\
\hline Time needed to fall asleep ( $\mathrm{min}$ ) & $21,3(6,20)$ & $17,5(3,39)$ & $14,4(3,49)$ & $17,3(3,93)$ & 0.85 & ns \\
\hline Number of awakenings & $1,4(0,92)$ & $0,3(0,18)$ & $0,6(0,27)$ & $0,5(0,32)$ & 1.01 & ns \\
\hline Sleep duration (h) & $6,9(0,31)$ & $7,3(0,23)$ & $6,9(0,31)$ & $7,3(0,21)$ & 1.44 & ns \\
\hline \multicolumn{7}{|l|}{ Bond and Lader questionnaire } \\
\hline Alertness & $66,2(5,71)$ & $67,6(6,17)$ & $63,0(6,93)$ & $80,4(2,87)$ & 3.65 & .019 \\
\hline Contentedness & $78,0(4,39)$ & $79,8(3,54)$ & $75,7(4,42)$ & $82,3(2,97)$ & 1.32 & ns \\
\hline Calmness & $81,5(2,99)$ & $83,5(2,76)$ & $76,1(4,34)$ & $84,7(2,46)$ & 2.36 & ns \\
\hline
\end{tabular}

ns= not significant 


\section{Discussion}

Data from the present study showed that escitalopram alone significantly impaired tracking performance in a divided attention task. Combinations of pindolol + escitalopram and ketanserin + escitalopram also impaired measures of divided attention when compared to placebo. The combination of escitalopram + ketanserin impaired sustained attention, even when compared to escitalopram alone. Subjective mood scales showed an overall reduction in alertness. It is not expected that the effects caused by the differential serotonergic manipulations were due to sedative properties, because the drug effects on the performance measurements of the different tasks, were very selective. If sedation was a causal factor, one would have expected more general impairment on all laboratory tasks. In addition, no correlations were found between degree of alertness and different measures of attention.

The lack of vigilance impairment after escitalopram alone is not in line with previous studies showing impairing effects of various SSRIs on vigilance performance using the same Mackworth Clock paradigm (Mackworth, 1950). This can probably be explained by the relative small number of subjects that were employed in the present study $(n=16)$. SSRI induced vigilance decrements are usually very minor and researchers regularly used up to 24 subjects in order to increase statistical power (Riedel et al., 2005). In the present study the number of correct detections after escitalopram decreased by only $6 \%$. When combined with pindolol the number of correct detection decreased by $13 \%$. The latter decrement did also not reach statistical significance. There was no interaction between treatment and time on task, indicating that the effect of the different treatments on vigilance decrement over time was relatively equal. This is in accordance with the abovementioned previous studies on serotonin and vigilance (Ramaekers et al., 1995; Schmitt, Ramaekers et al., 2002).

The combination of ketanserin + escitalopram decreased the number of correct detections by $33 \%$. This decrement not only differed from placebo but also from escitalopram alone. This strongly suggests that postsynaptic $5-\mathrm{HT}_{2 \mathrm{~A}}$ receptor blockade plays a modulatory role in vigilance performance. Various animal studies indicate that $5-\mathrm{HT}_{2}$ receptors modulate dopaminergic activity and that $5-\mathrm{HT}_{2 \mathrm{~A}}$ receptors and $5-\mathrm{HT}_{2 \mathrm{C}}$ receptors even exert opposite effects on dopamine release (Esposito, 2006). Dopamine release is inhibited by $5-\mathrm{HT}_{2 \mathrm{C}}$ receptor stimulation (Di Matteo et al., 2001; Lucas \& Spampinato, 2000) and dopamine is enhanced by $5-\mathrm{HT}_{2 \mathrm{~A}}$ receptor activation (Bortolozzi et al., 2005; Lucas \& Spampinato, 2000). Dopamine is released in the Ventral Tegmental Area (VTA) which leads to the mesocortical 
dopamine system (Bortolozzi et al., 2005). The mesocortical dopamine system in turn is regarded as an important modulator of cognition (Robbins, 2000). In the current study, $5-\mathrm{HT}_{2 \mathrm{~A}}$ receptor blockade by ketanserin may have decreased dopamine release which may have resulted in vigilance impairment.

There is more evidence that suggest a role for dopamine in attention. Simultaneous depletion of serotonin and catecholamines in healthy volunteers produced vigilance impairment independent of general drowsiness levels (Matrenza et al., 2004). According to the authors, the impairment in sustained attention was more likely to be caused by catecholamine reducement (norepinephrine and dopamine). However, some studies failed to demonstrate changes in vigilance performance after tyrosine depletion (Harmer et al., 2001; Harrison et al., 2004). Yet, dopamine suppletion following stimulant drugs such as methylphenidate and amphetamines improved sustained attention (Koelega, 1993; Nicholson \& Pascoe, 1990). In addition, as mentioned in the introduction, the SSRI sertraline did not impair vigilance performance (Riedel et al., 2005; Schmitt, Ramaekers et al., 2002) probably because it inhibits serotonergic as well as dopaminergic reuptake.

In the present study escitalopram and the combinations of pindolol + escitalopram and ketanserin + escitalopram all decreased tracking performance in a divided attention task. No effects of the specific serotonergic manipulations were found on selective attention. Impairments seen on the divided attention task after both combinations never differed from those observed after escitalopram alone, which seems to indicate that blockade of $5-\mathrm{HT}_{1 \mathrm{~A}}$ and $5-\mathrm{HT}_{2 \mathrm{~A}}$ receptors did not significantly contribute the impairments of divided attention. The impairment of tracking performance was evident in the divided attention task but not in the critical tracking task. The presence of impairment in the former task may be related tot the fact that this task involves performing two tasks simultaneously and this requires more attentional resources. Previous studies on the association between serotonin and selective or divided attention have produced mixed results. Serotonin suppletion following SSRI administration has usually not been associated with changes in selective and divided attention (Schmitt, Ramaekers et al., 2002; Wingen et al., 2005). Yet, tryptophan depletion generally improved performance in a Stroop task (Schmitt et al., 2000) and reduced motor speed on incompatible trials in a reaction time task (Coull et al., 1995).

With respect to the role of $5-\mathrm{HT}_{1 \mathrm{~A}}$ and $5-\mathrm{HT}_{2 \mathrm{~A}}$ receptors in attention, the results of the present study suggest that the $5-\mathrm{HT}_{2 \mathrm{~A}}$ receptor might be involved more 
in sustained attention as compared to divided or selective attention. As far as the 5$\mathrm{HT}_{1 \mathrm{~A}}$ receptor, it seems that this receptor does not have an additional effect next to a possible effect of general increased serotonin levels. However, the present study used $10 \mathrm{mg}$ pindolol to obtain a $37 \%$ binding of the $5-\mathrm{HT}_{1 \mathrm{~A}}$ autoreceptor. Still there can be a large variation in $5-\mathrm{HT}_{1 \mathrm{~A}}$ binding between subjects (Rabiner et al., 2004) and it is possible that not all subjects had an adequate $5-\mathrm{HT}_{1 \mathrm{~A}}$ receptor blockade to cause significant changes in serotonergic function. With less variation in binding, more specific changes in serotonergic function could occur and different effects on attention are possible. This is an important issue for future studies examining the role of different serotonergic receptors in cognition.

Motor impulsivity as assessed in the stop signal task was not affected by the specific serotonergic manipulations. Current notions on the role of serotonin in motor impulse control show different results. Serotonin suppletion following a single dose of 3,4-Methylenedioxymethamphetamine (MDMA) has previously been shown to decrease stop reaction time in a stop signal task (Ramaekers \& Kuypers, 2006) and motor impulse control decreases after central serotonin depletion (Harrison et al., 1997). Results from animal studies however are mixed and demonstrate both increments and decrements in motor impulsivity after $5-\mathrm{HT}_{1 \mathrm{~A}}$ receptor stimulation (Blokland et al., 2005; Carli \& Samanin, 2000). Possibly, serotonin as well as dopamine are involved in motor impulse control. It has been shown that stimulation of dopamine release will improve motor impulse control in a stop signal paradigm (de Wit et al., 2000; de Wit et al., 2002). The results of the present study on impulsivity cannot support previous findings, but possibly with a bigger sample size more firm statements can be given.

It is concluded that $5-\mathrm{HT}_{1 \mathrm{~A}}$ blockade hardly affected SSRI effects on attention. Combined $5-\mathrm{HT}_{2 \mathrm{~A}}$ blockade however produced additional impairment of sustained attention.

\section{Acknowledgments}

The authors are thankful to Silke Conen and medical doctor C.J. van Leeuwen, for their assistance in the data collection. 


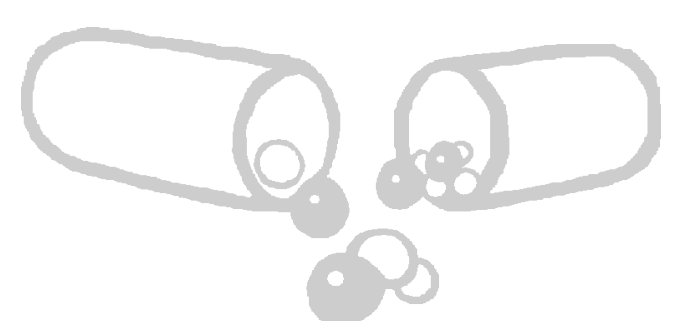

\title{
Chapter 8 Sustained attention and serotonin: a pharmaco- fMRI study
}

\begin{abstract}
Background: evidence suggests that stimulation of serotonergic function in healthy humans causes an impairment of sustained attention. Aim: the present study assessed the influence of increased serotonin levels on brain areas involved in sustained attention. Method: 10 healthy volunteers $(5$ ㅇ, $5 \hat{0})$ received the selective serotonin reuptake inhibitor (SSRI) escitalopram (20mg) and placebo in a balanced, double blind, 2-way crossover design. Participants performed the Mackworth Clock Test to measure sustained attention during functional MRI measurements at 3-Tesla. Subjective measurements after pharmacological manipulation were conducted with the Bond \& Lader Questionnaire. Results: independent of treatment, brain areas associated with task performance on a sustained attention task were activated, including right prefrontal and parietal areas. After escitalopram administration, less activation was shown in the caudate nucleus, thalamus and frontal areas. No effect of escitalopram was shown on behavioral data although subjective measurements showed decreased alertness after escitalopram. Conclusion: the results suggest that serotonin stimulation impairs sustained attention through modulating activation of selective brain areas including the thalamus and caudate nucleus, which are possibly involved in a subcortical network for sustained attention.
\end{abstract}

Wingen, M., Kuypers, K. P. C., Van de Ven, V., Formisano, E., \& Ramaekers, J. G. (2006). Sustained attention and serotonin: a pharmaco-fMRI study, submitted. 


\section{Introduction}

The neurotransmitter serotonin is involved in different psychiatric disorders, including major depression. The majority of patients diagnosed with a depressive disorder have a malfunction of the serotonergic system (Meltzer, 1989). Depression and also other psychiatric disorders are associated with cognitive dysfunction such as impaired memory performance and attention or planning deficits (Austin et al., 2001; Elliott, Baker et al., 1997; Landro et al., 2001). There is evidence that serotonin plays a role in the etiology of depression and is associated with cognitive problems (reviewed by (Schmitt et al., 2006)). Some studies in depressed patients showed that serotonin stimulation by antidepressants such as fluoxetine, fluvoxamine and trazodone, directly improves cognitive functions independent from elevation of depressive symptoms (Doraiswamy et al., 2003; Koetsier et al., 2002; Riedel, Schoenmakers et al., 1999). Studies in healthy volunteers have also provided evidence for a possible role of serotonin in cognitive performance. Low serotonin levels following tryptophan depletion and high serotonin levels following SSRI administration have both been shown to reduce memory performance (Riedel, Klaassen et al., 1999; Riedel, Klaassen, Griez et al., 2002; Wingen, Kuypers et al., 2006b). It has been suggested that the relation between serotonin and memory reflects an inverted U-curve (Meeter et al., 2006) which entails that both overstimulation and understimulation of serotonin levels worsen memory performance. Other cognitive functions such as cognitive flexibility and attention are possibly also modulated by serotonin (Schmitt et al., 2006). Particularly, sustained attention, often called vigilance, is one aspect of the general construct of attention that is associated with serotonergic functioning (Schmitt, Ramaekers et al., 2002). Single and repeated doses of SSRIs have been shown to reduce sustained attention in healthy volunteers in a number of studies using the Mackworth Clock Test paradigm (O'Hanlon et al., 1998; Ramaekers et al., 1995; Riedel et al., 2005; Schmitt, Ramaekers et al., 2002). While performing the Mackworth Clock Test, participants have to sustain their attention for 45 minutes while monitoring a circular arrangement of sequentially illuminating dots. A button press is required when a rare event occurs (signal detection) (Mackworth, 1950). As time passes, it becomes more difficult to sustain attention and more misses occur. This is called the vigilance decrement (Teichner, 1974).

Antidepressants are suitable means for challenging the serotonergic system while measuring cognitive functions. Moreover, combining (psycho)pharmacological studies with functional magnetic resonance imaging (fMRI) might give more insight 
into the underlying neuroanatomical substrates of task performance and changes in functioning of these brain areas associated with cognitive performance. Previous pharmaco-fMRI studies for establishing the association between serotonin and cognition have used several antidepressants. For instance, one study used the noradrenergic and specific serotonergic antidepressant mirtazapine to examine the effects of serotonin on behavioral inhibition in a parallel group design. The results showed a modulatory role for serotonin of brain responses in a Go/No-Go and a Reward/No-Reward task in various brain areas including orbitofrontal cortex and parietal cortex (Vollm et al., 2006). Unfortunately, mirtazapine has additional noradrenergic and histaminergic affinity, which can influence the results on the behavior level as well as on the neuroanatomic level. Ideally, a more selective serotonergic antidepressant is required. Another study used the selective serotonin reuptake inhibitor (SSRI) citalopram in a within-subject fMRI design and demonstrated effects of serotonin on brain areas involved in several neuropsychological tasks, despite little behavioral changes due to a ceiling effect (Del Ben et al., 2005). No prior pharmaco-fMRI studies have yet been undertaken to assess the effects of serotonin on sustained attention.

The aim of the present study was to define the brain regions that are involved in performance during the Mackworth Clock Test. In addition, the effects of increased serotonin levels in healthy human volunteers were assessed on sustained attention and on brain areas underlying sustained attention. Serotonin stimulation was obtained by blocking serotonin reuptake through administration of the most selective SSRI, escitalopram. It was expected that acutely increased serotonin levels would impair sustained attention performance measured by the Mackworth Clock Test. In addition, this impaired performance was expected to be reflected in activation changes in the brain areas that underlie sustained attention. Previous studies using sustained attention tasks and fMRI showed involvement of the right parietal and frontal brain areas (Coull et al., 1998; Lewin et al., 1996; O'Conner et al., 2004). It was expected that the same brain areas would be involved while performing the Mackworth Clock Test.

\section{Materials and Methods}

\section{Subjects}

Ten healthy volunteers (5, 5§), mean age (se) 26.3 (2.46) were recruited. All participants underwent a screening procedure, which consisted of a telephone 
interview, health questionnaire, 12-lead electrocardiogram, laboratory testing (hematology and blood chemistry, urinalysis, drug-and pregnancy screening) and a routine medical examination. Volunteers were included when they were 21 to 45 years of age, healthy, had a normal static binocular acuity (corrected or uncorrected), a body mass index between 19 and 30, and were willing to sign an informed consent. Excluded were those volunteers who suffered from, or had a history of cardiac, hepatic, renal, pulmonary, neurological, gastrointestinal, hematological or psychiatric illness. Other exclusion criteria were excessive drinking $(>20$ glasses of alcohol containing beverages a week), pregnancy or lactation, menstrual disorder, use of medication other than oral contraceptives, smoking tobacco or use of illicit drugs, and any sensory or motor deficits which could reasonably be expected to affect test performance. Those volunteers who had a first-degree relative with a psychiatric disorder or a history of a psychiatric disorder were also excluded. The study was approved by the standing medical ethics committee of Maastricht University and the Maastricht Academic Hospital's Board of Directors. It was carried out in accordance with the World Medical Association's Declaration of Helsinki (Edinburgh, 2000). Written informed consent was obtained from each volunteer prior to participation to the study.

\section{Design and Treatment}

The study was conducted according to a double-blind, placebo controlled, 2-way crossover design. Complete balancing of the treatments led to two treatment orders that were randomly assigned to the participants. Treatments consisted of escitalopram ( $20 \mathrm{mg}$ ) and placebo administered at 2 different test days separated by a wash-out period of at least 7 days.

\section{Testing procedure}

Participants underwent a short training session of the Mackworth Clock Test on the day of the medical examination in order to minimize learning effects. On test days, participants arrived at 9.00 a.m. at the laboratory, filled out an informed consent concerning scanning procedures, received a standard breakfast and completed a sleep quality questionnaire. They received the treatment capsule containing either escitalopram or placebo at 9.30 a.m. Participants were then seated for the next hours in a secluded waiting room in order to wait for escitalopram to reach the maximum concentration in blood (Cmax). At noon participants received a standard light lunch. 
Mood assessments were conducted at 13.00 p.m. followed by an anatomical scan. Scanning and testing took place at 13.30 p.m., i.e. 4 hrs after drug intake, till 14.15 p.m. Participants were not allowed to consume alcohol 24 hours prior to testing and caffeine-containing beverages 4 hours prior to the start of the test day.

\section{Subjective measurements}

Subjects filled out the Groninger Sleep Quality Scale (Mulder-Hajonides van der Meulen, 1981) on each test day to assess sleep quality during the preceding night. The total score consisted of 14 yes/no questions to score the number of sleep complaints (ranging from good sleep (score 0) to worst possible sleep (score 14)). In addition, specific questions on time needed to fall asleep, number of awakenings during the night and sleep duration in hours were included. The Bond and Lader Questionnaire was also assessed using different scales for Alertness, Contentedness and Calmness (Bond \& Lader, 1974).

\section{FMRI Data acquisition}

A 3 T Siemens Allegra MR scanner, situated at the Faculty of Psychology (University Maastricht, The Netherlands) was used for the anatomical and functional measurements. A T1-weighted anatomical scan was acquired for each subject using a 3-D MDEFT (Modified Driven Equilibrium Fourier Transform) sequence with an isotropic spatial resolution of $1 \mathrm{~mm}$. During task performance 896 whole-brain volumes of T2*-weighted functional measurements were acquired, each comprising 32 slices (slice thickness 3.5mm; no slice gap; flip angle $90^{\circ}$ ) using a blood oxygenation level dependent (BOLD) measurement and echo planar image (EPI) pulse sequence (TR: $2 \mathrm{sec}$; TE: $30 \mathrm{msec}$; resolution: $3.5 \times 3.5 \times 3.5 \mathrm{~mm}^{3}$, matrix size 64 x 64) and interleaved slice sampling. Stimulus presentation and scanning were synchronized at the beginning of the task.

\section{Sustained attention - Mackworth Clock Test}

FMRI scans were obtained while subjects performed the Mackworth Clock Test for 30 minutes. Participants laid supine in the scanner with the head fixated with foam pads. Head phone and earplugs were provided. Participants looked at the projection of a computer screen displaying a circular arrangement of 60 gray dots, via a mirror in the head volume coil. The dots were briefly illuminated in clockwise rotation at a rate of one per 500 milliseconds, moving 6 degrees from dot to dot. Volunteers were 
instructed that occasionally the dot would move 12 degrees. This 'skipping of one of the dots' was the signal participants had to detect throughout the task by pressing a response button as fast as possible with the right index finger. A response within 3 seconds of the signal was registered as a correct detection. A total of 20 signals were randomly presented with 10 per 15 minute period. Behavioral measures were total correct detections (hits) and corresponding reaction times. For objectives of a different study a resting condition was included prior to as well as proceeding to the task block. These additional time points were modeled as covariates in the current study.

\section{Statistical analyses}

Behavioral data were analyzed by means of a repeated measures general linear model (GLM) analysis (SPSS 11.5) with Treatment (2 levels) and Time on task (2 levels) as the main factors. The alpha criterion significance level was set at $\mathrm{p}=0.05$.

Preprocessing of the functional images was done using BrainVoyager QX version 1.6 (www.brainvoyager.com), and contained slice scan correction, 3D motion correction (trilinear interpolation) and linear trend removal and high pass temporal filtering (cut-off $=5$ cycles per time course). Individual anatomical datasets were spatially normalized to a standardized 3-D space (Talairach \& Tournoux, 1988). Individual scans were realigned using the first scan as a reference and coregistered and normalized to the anatomical data, and resampled to a voxel size of $3 \times 3 \times 3 \mathrm{~mm}^{3}$.

Analysis of the brain activations were performed using a whole-brain, fixed effects ANCOVA that included 12 predictors based on the task design. These predictors included correct detections, misses, false alarms, fixation, task block, time before correct detections and 6 motion correction preprocessing parameters. The predictor correct responses (corresponding to 2 time scans) was used as a main measure for sustained attention as previous studies showed an effect of increased serotonin levels on this variable (O'Hanlon et al., 1998; Ramaekers et al., 1995; Riedel et al., 2005; Schmitt, Ramaekers et al., 2002). The implication was that as a participant responds to the event (signal detection), it is also sustaining attention.

Results were color-coded and superimposed onto a standardized template of a single brain (courtesy of Montreal Neurological Institute (MNI)). Main task effects of placebo were visualized using a $\mathrm{p}$ (bonf) $<0.01$ corrected, and a cluster threshold of $>100$ voxels. The differential effect of escitalopram was analyzed using whole-brain analyses within the placebo-escitalopram contrast on the correct responses of the task using a q (FDR) <.001 (Genovese et al., 2002) (which corresponded to $p<0.000008$, 
uncorrected) and a cluster threshold of >50 voxels. Subsequent event-related averaging of the cluster time courses corresponding to the correct response trials was performed.

\section{$\underline{\text { Results }}$}

\section{Missing data}

In total, 12 participants were included of which two ended their participation due to claustrophobic reactions in the fMRI scanner. Ten participants completed the study, there were no missing data. Escitalopram was well tolerated in all subjects.

\section{Behavioural data}

Means and standard errors (se) of the sustained attention task performance are shown in Table 1.

Table 1 Mean (se) outcome variables of the Mackworth Clock Test and the Bond \& Lader questionnaire

\begin{tabular}{l||ll}
\hline $\begin{array}{l}\text { Treatment } \\
\text { N=10 }\end{array}$ & Escitalopram & Placebo \\
\hline \hline Mackworth Clock Test & $15.8(1.25)$ & $15.0(1.69)$ \\
Total correct responses & $629(32.4)$ & $662(38.3)$ \\
Total reaction time (msec) & $8.4(0.50)$ & $7.9(0.92)$ \\
Correct responses 0-15 min & $589(25.4)$ & $635(36.5)$ \\
Reaction time 0-15 min (msec) & $7.4(0.82)$ & $7.1(0.91)$ \\
Correct responses 15-30 min & $664(43.2)$ & $680(42.3)$ \\
Reaction time 15-30 min & & $87.4(3.96)$ \\
\hline Bond and Lader questionnaire & $79.0(4.41)$ & $83.4(4.05)$ \\
Alertness & $79.3(4.28)$ & $80.7(5.21)$ \\
\hline Contentedness & & \\
Calmness & & \\
\hline * p - 001 & & \\
\hline
\end{tabular}

* $=\mathrm{p}=.001$

Treatment did not significantly affect correct detections or reaction time $\left(\mathrm{F}_{1,9}=0.91\right.$, $\mathrm{p}=.366)$. There was a trend for a Time on task effect for correct detections $\left(\mathrm{F}_{1,9}=3.41\right.$, $\mathrm{p}=.098)$ and a significant Time on task effect for reaction time $\left(\mathrm{F}_{1,9}=9.61, \mathrm{p}=.013\right)$, which indicates an overall tendency for a vigilance decrement during the 30 minutes performance. There was no Treatment by Time on task interaction effect. 


\section{Subjective measurements}

Mean (se) of subjective evaluations are shown in Table 1. The outcome measures of the Groninger Sleep Quality Scale were not different for the two treatments. Treatment did significantly affect the Alertness ratings $\left(\mathrm{F}_{1,9}=21.5, \mathrm{p}=.001\right)$ but not the Contentedness or Calmness ratings. Escitalopram reduced alertness as compared to placebo.

\section{Functional MRI during task performance}

Figure 1 shows significantly activated brain areas in the placebo condition during the Mackworth Clock Test performance. The main effect of task showed increased activation of motor areas including the left post central gyrus, the right precentral gyrus, the middle cingulate gyrus, and the supplemental motor area. In addition there was increased activation in brain areas associated with an attentional network including the right inferior parietal gyrus, the right angular gyrus, the insula, the thalamus and the caudate nucleus. Other areas which showed increased activation were the middle frontal gyrus, the right middle temporal gyrus, the parietal-occipital fissure, the precuneus and the vermis. Decreased activation was seen in the posterior and anterior cingulate gyrus.

Figure 2 shows significantly activated brain areas after a contrast between escitalopram and placebo two conditions with corresponding event-related plots. Comparing escitalopram to placebo resulted in decreased activation of the left supplementary motor area, parts of the thalamus, the left caudate nucleus, the left precentral sulcus, the right middle frontal gyrus, the left inferior frontal gyrus and the left superior frontal gyrus. In addition, after escitalopram administration there was increased activation in the right superior temporal gyrus and the right lateral fissure. The event-related plots of the specific brain areas in the contrast between the two conditions showed less or no response (BOLD signal) after escitalopram administration. In table 2, significantly activated brain areas during Mackworth Clock Test performance are presented in the placebo condition and after a contrast between the two conditions (placebo-escitalopram) including mean $\mathrm{t}$-value and number of voxels. Figure 3 shows the event related plot of the primary motor cortex, which shows no difference between the two conditions. 
Table 2. Areas in which voxels were activated corresponding to correct detections of the Mackworth Clock Test

\begin{tabular}{|c|c|c|c|c|c|c|}
\hline \multirow[b]{2}{*}{ Region } & \multirow[b]{2}{*}{$\begin{array}{l}\text { Left/ } \\
\text { Right }\end{array}$} & \multicolumn{5}{|c|}{$\begin{array}{l}\text { Main effect (placebo) } \\
\text { Talairach coordinates }\end{array}$} \\
\hline & & $\mathbf{X}$ & $\mathbf{Y}$ & $\mathbf{Z}$ & $\begin{array}{l}\text { Number } \\
\text { of voxels }\end{array}$ & $\begin{array}{c}\text { Mean } \\
\text { t-value }\end{array}$ \\
\hline Postcentral gyrus/ Motor cortex + & $\overline{\mathrm{L}}$ & -30 & -30 & 50 & 945 & 7.725 \\
\hline Middle cingulate gyrus + & $\mathrm{L} / \mathrm{R}$ & 1 & 3 & 36 & 1331 & 10.248 \\
\hline Supplementary motor area + & $\mathrm{L} / \mathrm{R}$ & 2 & 12 & 51 & 1321 & 9.043 \\
\hline Inferior parietal gyrus + & $\mathrm{R}$ & 40 & -47 & 42 & 1045 & 8.771 \\
\hline \multirow[t]{2}{*}{ Precentral gyrus + } & $\mathrm{R}$ & 41 & -6 & 41 & 963 & 7.494 \\
\hline & $\mathrm{R}$ & 46 & 3 & 24 & 1274 & 7.946 \\
\hline Angular gyrus + & $\mathrm{R}$ & 49 & -43 & 31 & 1211 & 9.330 \\
\hline Middle temporal gyrus + & $\mathrm{R}$ & 53 & -43 & 9 & 1160 & 8.336 \\
\hline Insula + & $\mathrm{L} / \mathrm{R}$ & 35 & 18 & 7 & 1331 & 11.139 \\
\hline Thalamus + & $\mathrm{L} / \mathrm{R}$ & 9 & -16 & 10 & 1070 & 8.015 \\
\hline Caudate nucleus + & $\mathrm{L} / \mathrm{R}$ & 9 & 5 & 9 & 1133 & 7.247 \\
\hline Middle frontal gyrus + & $\mathrm{L} / \mathrm{R}$ & 37 & 37 & 24 & 835 & 6.064 \\
\hline Parietal-occipital fissure + & $\mathrm{L} / \mathrm{R}$ & 10 & -70 & 28 & 735 & 6.349 \\
\hline Precuneus + & $\mathrm{L} / \mathrm{R}$ & 1 & -53 & 35 & 645 & 6.234 \\
\hline Vermis + & $\mathrm{L} / \mathrm{R}$ & 2 & -53 & -19 & 1317 & 8.565 \\
\hline Posterior cingulate gyrus - & $\mathrm{L} / \mathrm{R}$ & -3 & -54 & 17 & 652 & -6.055 \\
\hline Anterior cingulate gyrus - & $\mathrm{L} / \mathrm{R}$ & -3 & 31 & -7 & 157 & -5.455 \\
\hline
\end{tabular}

$+=$ increased activation

- = decreased activation

\begin{tabular}{|c|c|c|c|c|c|c|}
\hline \multirow[b]{2}{*}{ Region } & \multirow{2}{*}{$\begin{array}{l}\text { Left/ } \\
\text { Right }\end{array}$} & \multicolumn{5}{|c|}{$\begin{array}{c}\text { Different activation after escitalopram } \\
\text { administration } \\
\text { Talairach coordinates }\end{array}$} \\
\hline & & $\mathbf{X}$ & $\mathbf{Y}$ & $\mathbf{Z}$ & $\begin{array}{l}\text { Number } \\
\text { of voxels }\end{array}$ & $\begin{array}{c}\text { Mean } \\
t \text {-value }\end{array}$ \\
\hline Supplementary motor area - & $\overline{\mathrm{L}}$ & $\overline{-6}$ & 11 & $\overline{48}$ & 333 & -4.972 \\
\hline Ventral nucleus left - & $\mathrm{L}$ & -8 & -18 & 9 & 54 & -4.649 \\
\hline Mediodorsal nucleus left - & $\mathrm{L}$ & -5 & -7 & 9 & 209 & -4.991 \\
\hline Mediodorsal nucleus right - & $\mathrm{R}$ & 7 & -13 & 11 & 176 & -4.847 \\
\hline Anterior nucleus - & $\mathrm{L} / \mathrm{R}$ & 0 & -5 & 7 & 372 & -5.021 \\
\hline Caudate nucleus - & $\mathrm{L}$ & -13 & 4 & 14 & 262 & -4.796 \\
\hline Precentral sulcus - & $\mathrm{L}$ & -24 & -8 & 51 & 299 & -4.975 \\
\hline Middle frontal gyrus - & $\mathrm{R}$ & 32 & 16 & 43 & 79 & -4.824 \\
\hline Inferior frontal gyrus - & $\mathrm{L}$ & -34 & 31 & 28 & 183 & -5.072 \\
\hline Superior frontal gyrus - & $\mathrm{L}$ & -27 & 53 & 15 & 67 & -4.731 \\
\hline Superior temporal gyrus + & $\mathrm{R}$ & 53 & 4 & 4 & 76 & 4.763 \\
\hline Lateral fissure + & $\mathrm{R}$ & 61 & -19 & 15 & 77 & 4.856 \\
\hline
\end{tabular}

$+=$ significant increased activation compared to placebo

- = significant decreased activation compared to placebo 
Figure 1 Main task effect of the Mackworth Clock Test (correct detections) in the placebo condition, increased and decreased activations are both presented at a $\mathrm{p}($ bonf $)<0.01$ and a cluster threshold of $>100$ voxels

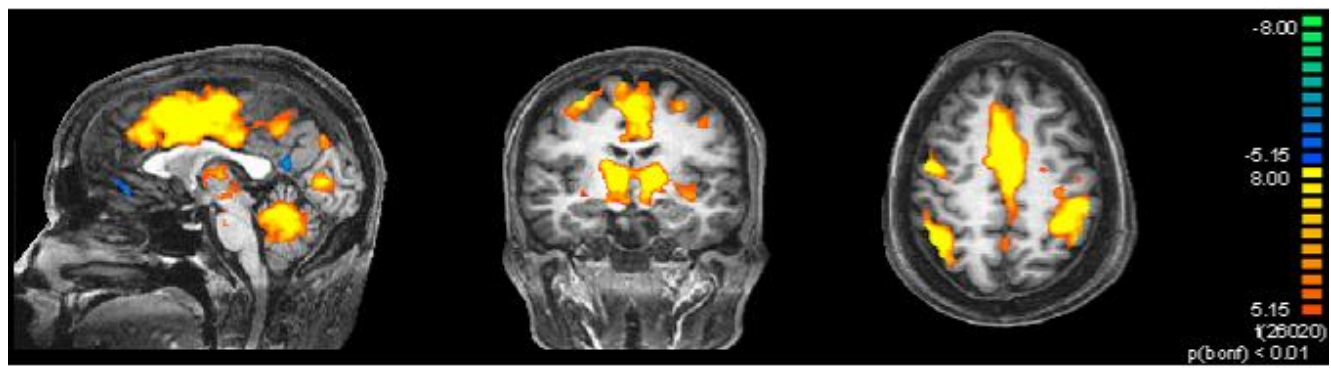

Figure 2 Decreased activation after the placebo-escitalopram contrast at q (FDR) < .001 and a cluster threshold of $>50$ voxels

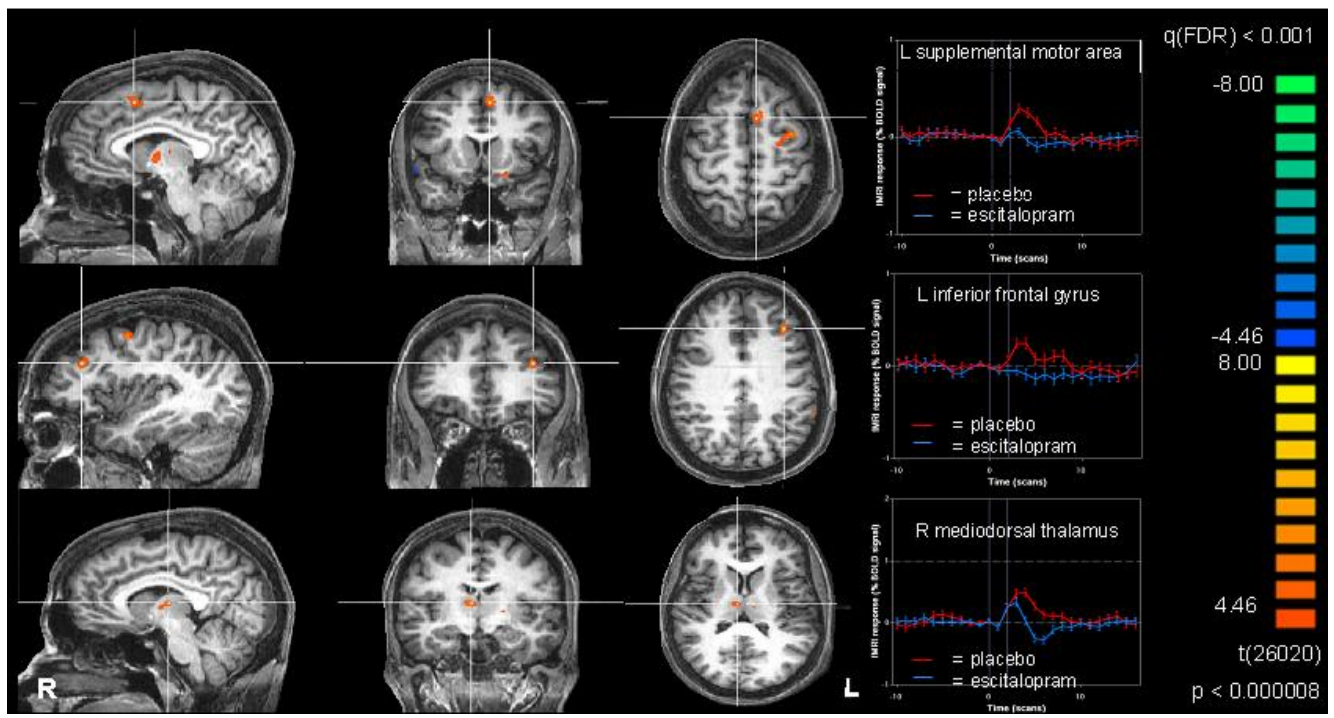


Figure 3 Event related plot in the primary motor cortex which shows no difference between escitalopram and placebo administration

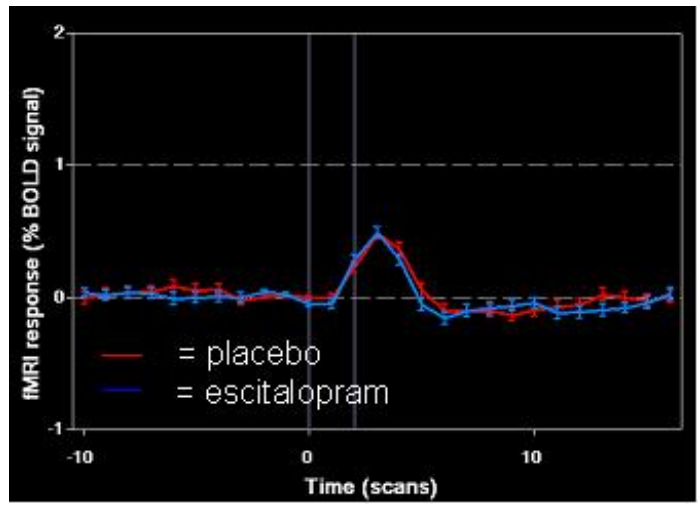

\section{Discussion}

The present study examined the effects of increased serotonin levels on sustained attention and underlying brain activation. Similar to previous studies that measured sustained attention (Coull et al., 1998; Lewin et al., 1996; O'Conner et al., 2004) the current study showed activation of right dorsal and prefrontal areas. The brain areas of the present study involved in sustained attention show some remarkable overlap with brain areas associated with visual attention (Corbetta et al., 2002). In addition, escitalopram and consequently the neurotransmitter serotonin modulated activity of several brain areas including the thalamus and prefrontal areas during a sustained attention task, although no effect was found on behavioral measurements of sustained attention.

Performance of the Mackworth Clock Test in the placebo condition showed increased activation in the right inferior parietal gyrus, the right angular gyrus, the right precentral gyrus, the insula bilateral, the thalamus bilateral, and the bilateral middle frontal gyrus. These areas and particularly right parietal and prefrontal areas have been associated with sustained attention in previous research with fMRI (Cabeza \& Nyberg, 2000; Sturm \& Willmes, 2001) and PET (Positron Emission Tomography) (Mottaghy et al., 2006). Using the rapid visual information (RIVP) task to assess networks of sustained attention, Lawrence et al. (2003) demonstrated a network comprising frontal and parietal cortical areas and the thalamus and caudate nucleus, which is very comparable to the set of active brain areas found in the present study (Lawrence et al., 2003). Coull (1998) suggests that two different networks are 
involved in attention and arousal and interact with each other: a cortical network including the right frontal and inferior parietal cortex, which is associated with attention and a subcortical network including the thalamus, striatum and the anterior cingulate, which is associated with arousal (Coull, 1998). Adequate levels of both arousal and attention are necessary to achieve good sustained attention performance (Coull, 1998). Other areas with increased activation in the present study were areas associated with the response by a button press including the left postcentral gyrus/motor cortex and the supplemental motor area. Activation of the parietaloccipital fissure is probably related to the seeing of the signal in the Mackworth Clock Test, as this area has been previously associated with perception of visual motion (Richer et al., 1991).

After escitalopram administration, differences in activation were seen in several brain areas. Decreased activation was seen in parts of the basal ganglia, including the ventral lateral nucleus of the left thalamus, the mediodorsal nucleus of thalamus bilateral and the left caudate nucleus. In addition, the left superior precentral sulcus, the left superior frontal gyrus and the right middle frontal gyrus also showed decreased activation after acutely elevated serotonin levels. The eventrelated plots of these areas also showed lower amplitude of activity and/or a postevent dip in activation for the escitalopram administration condition in the abovementioned areas. These decreased activations did not appear in the motor cortex, there were no differences between the two conditions in the primary motor cortex and also the event-related plot of the primary motor cortex showed an equal BOLD response after escitalopram and placebo. This suggests that the observed effects may be related to cognitive effects, possibly sustained attention.

Several nuclei of the thalamus including the ventrolateral and mediodorsal nuclei, in which decreased activity is found after escitalopram administration in the present study, are involved in mediating the interaction between attention and arousal in humans (Portas et al., 1998). The findings of the present study suggest that increments in serotonin levels impair sustained attention through influencing the interaction between the cortical and subcortical network involved in arousal and attention, described previously by Coull et al (1998). A candidate mechanism of the influence of serotonin on selective changes in brain activity may be through a serotonin-dopamine interaction mechanism. The basal ganglia (including the thalamus and the caudate nucleus) are often associated with the neurotransmitter dopamine (Garnett et al., 1983) and it has previously been proposed that serotonin 
has a general inhibitory effect on dopamine release (Soubrie, 1986; Spoont, 1992). Particularly serotonin projections from the raphe nucleus have an inhibitory influence over the mesocortical dopamine system and forebrain, which are involved in cognition (Kapur \& Remington, 1996). It could be suggested that serotonin impairs sustained attention through inhibiting dopamine in the thalamus and the caudate nucleus and consequently a decrease in dopamine may lead to less activation of the mesocortical dopamine projections to the prefrontal cortex (Robbins, 1997). The results from the present study support this statement by showing decreased activity in the thalamus as well as in (pre)frontal areas after serotonin stimulation. In humans and in primates, connections between the mediodorsal nuclei of the thalamus and the prefrontal cortex are well demonstrated (Behrens et al., 2003; Siwek \& Pandya, 1991) and these connections are involved in top-down modulation of attention (La Berge, 1995).

The present study did not show an effect of increased serotonin stimulation on behavioral measurements of attention. However, other studies did find an impairing effect of serotonin on sustained attention measured by the Mackworth Clock Test (O'Hanlon et al., 1998; Ramaekers et al., 1995; Riedel et al., 2005; Schmitt, Ramaekers et al., 2002). The differences between the previous studies and the present study are probably due to a small sample size and a small effect size. In addition, in the present fMRI study, the task only lasted 30 minutes whereas it lasted 45 minutes in performance studies. Possibly, the task duration was too short to pick up any significant differences between the treatment conditions, which is supported by our finding of a non-significant trend of a vigilance decrement. Escitalopram did decrease alertness subjectively but this was not reflected in behavioral measures of sustained attention. In addition, the entire procedure of conducting the task in the scanner may have influenced performance in that both treatments affect sustained attention in a comparable way. The number of correct detections after 30 minutes in the placebo condition in other (not-fMRI) studies is around 17.4 (se +/-2.0) (Ramaekers et al., 1995; Wingen, Kuypers et al., 2007). In the present study the number of correct detections in the placebo condition is certainly lower, 15.0 (se+/ - 1.8).

An important issue is the influence of drug administration on general blood flow. In fMRI, activations measured are an indirect reflection of neural activity but a direct reflection of the difference between the magnetic properties of oxygenated and deoxygenated hemoglobin or BOLD response. Theoretically, it is possible that serotonin influenced blood flow or BOLD changes in the present study. However, 
activations seen in the current study are task dependent and specific. This is in line with a previous study using intravenous administration of the SSRI citalopram that demonstrated that citalopram is a good tool to use in pharmacoMRI studies to manipulate the serotonergic system (McKie et al., 2005). Furthermore, the present study showed increased activations as well as decreased activations of BOLD response after increased serotonin levels in the same task manipulation. Activations in opposite direction are not possible in the context of a general effect on blood flow. Finally, SSRIs in general have few cerebrovascular effects (Ramasubbu, 2004) and particularly $20 \mathrm{mg}$ of the parent compound of escitalopram (citalopram) did not alter heart rate in healthy males (Seifritz et al., 1996). Therefore, it is unlikely that general effects on the BOLD response caused by escitalopram influenced the results.

For future studies it would be very interesting to use a data-driven method such as independent component analysis (ICA) (McKeown \& Sejnowski, 1998) to indicate different components or functional networks related to sustained attention. The decreased activity demonstrated in the anterior and posterior cingulate cortex in the present study is a remarkable finding to further examine with ICA as these areas seem to be involved in a certain network concerning resting and are shown to decrease when the cognitive demand of a certain task increases (Raichle et al., 2001). Furthermore, the use of this method would allow us to better attribute the influence of increased serotonin on sustained attention to a certain neural network.

To conclude, it could be suggested that serotonin stimulation may impair sustained attention through modulation of selective brain areas including (pre)frontal areas and parts of the basal ganglia, which are possibly involved in a subcortical network for sustained attention.

\section{Acknowledgments}

The authors are thankful to Sven J.C. Gijsen for his assistance in scanning procedures and data collection and medical doctor Cees J. van Leeuwen for his medical assistance. 


\section{Chapter 9}

\section{General Discussion \\ Discussion}
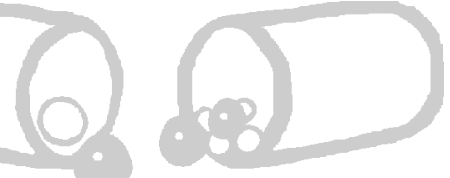

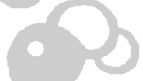

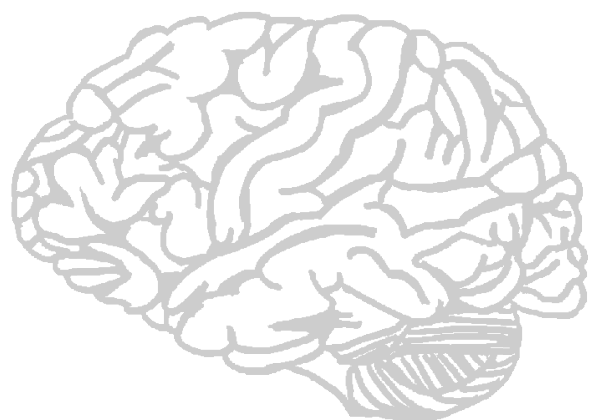


Depression and the use of antidepressants are an important concern for the patient and the society. Research in the present thesis examined antidepressant effects on cognitive functions and driving performance in healthy volunteers as well as in depressed patients. Furthermore, the hypothesis that the neurotransmitter serotonin is involved in different aspects of behaviour (including mood) and cognition was investigated. The involvement of serotonin in cognition (specifically memory and attention) was investigated by manipulating serotonin availability and measuring its effects on specific brain mechanisms and comparing between drugs affecting different 5-HT receptors. In this last chapter, it is attempted to relate the outcomes from the different chapters, to give an overview and to discuss the findings in a broader perspective. First, the main results of the different chapters will be considered in the light of the aim of the thesis. Next, it will be discussed how the results of this thesis contribute to previous research on depression, antidepressants, serotonin and cognition. Furthermore, methodological issues in experimental psychopharmacological studies will be discussed and implications will be given for future psychopharmacological research. Finally some concluding remarks will close this chapter.

\section{Main results}

The present thesis contains two types of experimental psychopharmacological studies: applied studies and fundamental studies. The aim was firstly to give more information on side effects of antidepressants. The two applied studies, described in Chapter 3 and 4, assessed the side effects of antidepressants on cognition and driving performance, in healthy volunteers and in depressed patients. In healthy volunteers, the SSRI escitalopram did not influence psychomotor function or driving performance, but the noradrenergic and serotonergic antidepressant mirtazapine impaired actual driving performance (increase in Standard Deviation of Lateral Position or SDLP), produced an increase in tracking error on a divided attention task and caused decreased subjective alertness. Both antidepressants were administered in the evening and mirtazapine's detrimental effects were only seen after the initial dose. Subchronic use did not influence psychomotor function or driving performance. Impairing effects of mirtazapine can be attributed to histaminergic $\mathrm{H}_{1}$ receptor binding, which causes drowsiness or sedation. In depressed patients, after long-term use of commonly administered antidepressants such as SSRIs and the SNRI venlafaxine driving performance was impaired (increased SDLP and Time to Speed 
Adaptation) compared to healthy volunteers. The use of these antidepressants had no effects on laboratory tests of cognition in the depressed patients. Driving impairment after long-term SSRI and SNRI use by depressed patients is somehow unexpected given that SSRIs are generally known to produce no or little effects on psychomotor function and cognition in healthy volunteers compared to sedative antidepressants (Ramaekers, 2003) as also was demonstrated in Chapter 3. In addition, it is known that depressed patients receiving antidepressant treatment will experience difficulties in performance particularly at the beginning of the treatment before the onset of therapeutic response, based on the side effects of the medication and the depressive symptoms. After a few weeks, when the treatment provides an alleviation of depressive symptoms, there will be a balance between side effects and beneficial effects of the treatment (Lane \& O'Hanlon, 1999). Consequently, it is unlikely that side effects of the antidepressants influenced driving performance in our study and therefore most likely the impairing effects are caused by residual depressive symptoms which were still present. However, other studies are necessary to draw firm conclusions.

The second aim of the present thesis was to explore the role of serotonin in cognition. The fundamental studies of this thesis described in Chapter 5, 6, 7 and 8 were preceded by a review discussing previous knowledge on serotonin and cognition. There is a role for serotonin in several cognitive functions, particular in human episodic memory, attention and cognitive flexibility. Evidence can be found mainly in challenge studies which use acute tryptophan depletion or SSRIs but there are inconsistencies concerning the direction of experimentally induced serotonin decrease and increase and its association with either cognitive enhancement or impairment. Regarding (elderly) depressed patients, serotonin seems to have beneficial effects on memory (Butters et al., 2000) and studies in depressed patients but also in aging would provide further insight in these issues. In Chapter 5, the effects of acute and subchronic administration of the SSRI escitalopram and the $3^{\text {rd }}$ generation antidepressant mirtazapine were examined on verbal memory. In fact, this chapter describes the effects of two different antidepressants on verbal memory (applied research) on the one hand but provides more information on serotonin and memory (fundamental research) on the other hand. The results of this study showed a slight impairing effect of mirtazapine on verbal memory probably due to its $\mathrm{H}_{1}$ receptor blocking properties. No effects of acute and subchronic administration of escitalopram and consequently of serotonin on verbal memory were demonstrated. 
Unfortunately, there was no measurement of the earliest action of escitalopram because the antidepressants were administered in the evening. Conversely, the following chapters described the effects of acutely manipulated serotonin levels on different aspects of cognition. Escitalopram tended to impair immediate verbal recall and further increased serotonin levels by means of combining an SSRI and a 5- $\mathrm{HT}_{1 \mathrm{~A}}$ autoreceptor antagonist significantly impaired immediate verbal memory. Together with previous work (Meeter et al., 2006), the present results support an association between serotonin and memory that describes an inverted U-curve. This effect is specific for verbal memory because additional increased serotonin levels through 5$\mathrm{HT}_{1 \mathrm{~A}}$ receptor blockade did not impair spatial memory and hardly affected different aspects of attention. The 5- $\mathrm{HT}_{2 \mathrm{~A}}$ receptor appears to be involved in spatial memory, as blockade of this receptor in combination with escitalopram, impairs performance on a spatial memory task. Furthermore, sustained attention is also modulated by the $5-\mathrm{HT}_{2 \mathrm{~A}}$ receptor and probably a link between the 5- $\mathrm{HT}_{2 \mathrm{~A}}$ receptor and dopamine release is responsible for these effects on cognition (Bortolozzi et al., 2005). More information on an interaction between serotonin and dopamine associated with sustained attention comes from the fMRI study in this thesis, in which increased serotonin levels on brain mechanisms involved in the Mackworth Clock Task were explored. After increased serotonin levels, less activation was found in the caudate nucleus, thalamus and (pre)frontal areas. This suggests that serotonin modulates sustained attention via a subcortical network including the thalamus from which dopaminergic pathways leading to (pre)frontal areas are also influenced.

\section{Side effects of antidepressants on cognition and driving performance}

Side effects of antidepressants as sedation, blurred vision or dizziness, interact with daily life and may impair cognition and driving performance. Sedative antidepressants including tricyclic antidepressant impair driving performance in healthy volunteers, but SSRIs cause less impairing effects (Ramaekers, 2003). Epidemiological research shows that TCAs increase the risk of becoming involved in a traffic accident (Leveille et al., 1994) (Ray et al., 1992) but SSRIs do not (Barbone et al., 1998). Recently, a clinical study in depressed patients using a computerized testsystem assessing driving skills confirmed these results (Brunnauer et al., 2006). Preferably, severe major depression should be treated with effective antidepressants with few side effects. 
One novel finding described in this thesis is that escitalopram of which is said that it is currently the most selective SSRI (Owens et al., 2001) had no influence on actual driving performance and psychomotor function after an evening dose. In addition, a nocturnal dose $(30 \mathrm{mg})$ of the sedative antidepressant mirtazapine did impair driving performance and psychomotor function the day after initial treatment, in spite of the fact that previous studies suggested that the considerably impairing acute sedative effect of daytime-administered mirtazapine $(15 \mathrm{mg})$ might much be alleviated by noctural administration (Ramaekers et al., 1998) . This information may be valuable for medical practice as mirtazapine is often prescribed at a start dosage of $30 \mathrm{mg}$. Considering the effects of antidepressant use in depressed patients, various studies show impairing effects on measurements related to driving behaviour (Gerhard \& Hobi, 1984) (Grabe et al., 1998) and also on actual driving performance (Ramaekers et al., 1997). In other words, antidepressant treatment of maximally 6 weeks did not normalize performance in depressed patients. This thesis adds to this that even after long-term antidepressant treatment (SSRIs and SNRI), i.e. 6 till 52 weeks, driving performance in depressed patients is still impaired compared to healthy persons.

An important issue is the correspondence of cognition and driving performance in terms of information processing demands. Driving behaviour contains aspects varying from automatic (fast and inflexible) processing to effortful (slow and flexible) processing. The actual driving tasks used in the research of this thesis measured driving performance on the more automatic operational level (weaving) and on the manoeuvring level (car-following) (Lamers \& Ramaekers, 2001). Other aspects of driving behaviour including planning, understanding and applying traffic rules occur at the strategic level and are not measured with the driving tests used in the present thesis. Laboratory tasks are supposed to measure different aspects of driving related behaviour. More specific, the divided attention task, a double task containing a signal detection subtask and a tracking subtask, seems to be closely related to the Road Tracking Test, for example the tracking component is very similar to keeping a car on the road. Nevertheless, results of the tracking task correlate only modestly with SDLP measurements and other psychomotor tests do not correlate with these results of driving performance (mean $\mathrm{r}$ of 0.23 - 0.45) (Ramaekers, 2003). Another inconsistency is that SSRIs impair sustained attention or vigilance in the Mackworth Clock Test, but SSRIs have few effects on driving performance as the Road Tracking Test, although both tasks may be seen as vigilance tasks. A possible explanation is that the Mackworth Clock Test 
measures only sustained attention by signal detection and reaction time but the Road Tracking Test measures various variables including Standard Deviation of Lateral Position and speed variation. The Mackworth Clock Test is designed to mainly measure vigilance while the road tracking task also measures other aspects of driving performance. Importantly, an actual driving test also includes selfmonitoring of arousal and performance which can be seen as an executive function. While driving in a real car there is the knowledge of the possibility of getting involved in an accident. This component is not or to a lesser extent existing in laboratory tasks and this may account for different results. Obviously the two types of tasks are not directly comparable and possibly different processes are measured on different levels. Apparently, the character of the task in terms of its cognitive demands is of great importance to assess psychopharmacological effects. From this point of view, actual driving tasks are of important additional value as cognitive tasks are limited in predicting the effects of drugs on daily life.

\section{Role of serotonin in cognition}

Animal research previously showed that serotonin receptors in the brain are situated in structures associated with memory function such as the hippocampus. Moreover, memory is influenced by 5-HT agonists or antagonists that act on different serotonergic receptors (Buhot et al., 2000) (Meneses, 1999). Evidence exists that 5$\mathrm{HT}_{1 \mathrm{~A}}$ and $5-\mathrm{HT}_{2}$ receptors are involved in memory. The present thesis showed for the first time that human $5-\mathrm{HT}_{1 \mathrm{~A}}$ and $5-\mathrm{HT}_{2 \mathrm{~A}}$ receptors are distinctly involved in verbal memory and spatial memory respectively. Furthermore, the results are consistent with the notion that $5-\mathrm{HT}_{2 \mathrm{~A}}$ receptors modulate dopaminergic activity (Bortolozzi et al., 2005) and thereby influence aspects of cognition as spatial memory and sustained attention. This is in line with findings reported in a very recent review on the mechanisms of serotonin in regulating dopamine neurotransmission (Alex \& Pehek, 2006). Evidence is available that activation of the $5-\mathrm{HT}_{2 \mathrm{~A}}$ receptor increases dopaminergic activity in all three dopaminergic pathways, i.e. nigrostriatal, mesolimbic and mesocortical pathway. Furthermore, selective antagonism of 5- $\mathrm{HT}_{2 \mathrm{~A}}$ receptors diminishes dopamine release (Alex \& Pehek, 2006). Consequently, this suggests that a decrease in dopamine activity by blockade of the $5-\mathrm{HT}_{2 \mathrm{~A}}$ receptor may lead to cognitive impairment as was shown in the present thesis. Since the present thesis manipulated serotonin, no direct conclusions can be drawn on dopamine or an interaction between dopamine and serotonin and therefore future research is needed. 
In addition, the present thesis for the first time provides more evidence for a role of serotonin in sustained attention by showing less activity in brain areas including thalamus, caudate nucleus and prefrontal areas while performing the Mackworth Clock Test after SSRI administration. Two different networks, one subcortical for arousal and one cortical for attention, are involved in sustained attention and these networks interact with each other (Coull, 1998). Particularly the thalamus is involved in mediating the interaction between attention and arousal in humans (Portas et al., 1998). The results of the present thesis suggest, together with previous research, that an SSRI impairs sustained attention by affecting the interaction between the subcortical and cortical network in the thalamus.

Altogether, the relation between serotonin and cognition is complex and not only mediated by serotonin levels but also by receptor mechanisms and the interaction with other neurotransmitters. Previous research showed a role for serotonin in cognitive flexibility (Murphy et al., 2002), motor impulse control (Ramaekers \& Kuypers, 2006) and selective attention (Coull et al., 1995; Schmitt et al., 2000) and also impulsivity (Chamberlain et al., 2006).The fundamental studies in this thesis cannot confirm these findings and future studies could examine the role of different serotonin receptors in other aspects of cognition. In addition, other 5-HT receptors may also be involved in cognition including learning and memory given the number and diversity of serotonin receptors found in brain areas associated with learning and memory (Buhot et al., 2000; Meneses, 1999). Recent evidence from animal studies suggest even cognition enhancing effects after $5-\mathrm{HT}_{4}$ or $5-\mathrm{HT}_{6}$ receptor manipulations (Micale et al., 2006; Schreiber et al., 2006).

\section{Methodological issues in psychopharmacology and recommendations for future} research

In the applied studies, a typical design to assess the effects of antidepressant on driving performance is a placebo-controlled within subjects design. However, the study conducted in depressed patients in this thesis deviates from this by studying driving performance in a between subject design. The SDLP of different groups (depressed patients using antidepressants vs healthy controls) were compared whereas it is challenging to compare driving behaviour of different persons. By matching the groups on several variables including age, gender and driving experience and by only allowing participants with a driving license for at least three years and driving experience of at least $5000 \mathrm{~km}$ /years, differences between groups 
are minimized and this may be a good design for studying between-subject driving performance. However, there will be other variables influencing driving performance except from age, gender and driving experience, for example personality or risk taking behaviour. For this reason, a within subject-design will be better when there is the possibility to use this study design.

Furthermore, after examining the effect of antidepressant treatment on driving performance of depressed patients, the question remains if antidepressant treatment may attenuate even more severe driving impairment after untreated depression. Additional analysis in the present thesis suggests that there may be a small beneficial effect of treatment on driving performance. This is in line with recent results of a study in which crash-involved drivers filled out a questionnaire on health and use of medication. The chance to be involved in a traffic accident was greater for persons with depressed feelings than for persons who take antidepressant drugs (Sagberg, 2006). The development of driving performance of depressed patients without and with antidepressant treatment over time is an important topic for further investigation in an experimental within-subject design. In addition, applied studies measuring the effects of antidepressants on driving performance could be more elaborated by measuring more aspects of driving behaviour than currently is conducted. By measuring driving behaviour on different levels and also on the strategic level, which includes the reaction of a participant to traffic signs, in experimental research, it should be possible to draw even more conclusions on accident risk and participating in traffic in daily life.

In the studies described in this thesis, relatively new methods are applied in humans. This thesis contains the first ever publication of a human study in which cognition was examined while different specific serotonergic drugs were administered in combination, with the aim to elevate synaptic 5-HT availability while also blocking specific (post-)synaptic 5-HT receptors. The present thesis showed that these are promising methods in psychopharmacology but it has to be emphasized that the use of selective agents with no other side effects is very important to provide information on the role of specific receptors in cognition. In addition, manipulation of the serotonergic system in depressed patients may lead to further insight in the role of serotonin in cognition as this can give additional information to studies in healthy volunteers. For example, more room is available for improvement of cognitive functions after serotonergic stimulation in depressed patients. Another main issue when studying the role of serotonin in patients is if cognitive improvement is directly 
due to enhanced serotonin levels or if the improvement of cognition is due to alleviation of depressive illness. Studies that compare cognitive performance of healthy volunteers and depressed patients after administration of a selective agonist or antagonist can give more insight in these issues. For example, in a previously conducted study (Riedel et al., 2002) ipsapirone, a 5-HT $1 \mathrm{~A}$ agonist, tended to improve short-term memory in depressed patients but impaired short-term memory in healthy volunteers, independent of mood changes. These results suggest a role for serotonin in memory but differently between patients and healthy volunteers. Future studies may investigate the role of serotonin in cognition at the receptor level in healthy volunteers as well as in depressed patients.

Furthermore, the use of fMRI in psychopharmacological research is also a relative new method. Functional imaging methods in psychopharmacology will help identifying neural circuits involved in drug effects on cognition and performance. However, knowledge obtained in pharmaco-fMRI studies provides only indirect clues about how drugs may modify cognitive task related BOLD activation. Pharmaco-fMRI's best value therefore may be to close the gap between prior knowledge obtained at the neurotransmitter level, i.e. about cellular mechanisms of drugs and obtained at the behavioural level such as changes in cognitive performance induced by these drugs. More information on the role of different serotonin receptors in cognition could be obtained by methods as PET and SPECT in which specific receptors can be anatomically mapped. Brain imaging in general together with the use of selective agents to get more insight in underlying brain mechanisms is an appealing method to use in healthy volunteers as well as patients which will receive more publicity in the future.

\section{Concluding remarks}

Taking into account the results described in the present thesis, some conclusions and implications can be given. Firstly, the adverse side effects of antidepressants due to additional mechanisms next to their therapeutic action may have an impact on the life and performance of patients. Therefore it is important to test these drugs on negative cognitive effects. This may of course be done in a laboratory with computerized tasks, although to predict performance in daily life as much as possible, actual driving tests should be included and more experimental studies in patients should be conducted.

Secondly, the core therapeutic action of antidepressants relies on serotonin. Using selective antidepressants in fundamental research to manipulate serotonin can 
provide more insight in negative or positive effects on cognitive functions. Furthermore, selectively manipulating 5-HT receptors and also using pharmaco-fMRI in human studies may give even more information on the underlying mechanisms of serotonin in cognition. The present thesis is the first to show that the $5-\mathrm{HT}_{1 \mathrm{~A}}$ receptor is involved in verbal memory and that the $5-\mathrm{HT}_{2 \mathrm{~A}}$ receptor is an important receptor involved in spatial memory and sustained attention. Furthermore, the impairing effect of SSRIs on sustained attention is visualized in the brain. These findings have opened new doors for future experimental research that concerns neurotransmitters and cognition. 


\section{References}

Aellig, W. H., Narjes, H. H., Nuesch, E., Oertle, R. J., Devos, J. E., \& Pacha, W. (1981). A pharmacodynamic and pharmacokinetic comparison of pindolol $20 \mathrm{mg}$ retard and a conventional tablet. European journal of clinical pharmacology, 20(3), 179-183.

Aghajanian, G. K., \& Sanders-Bush, E. (2002). Serotonin. In K. L. Davis, D. Charney, J. T. Coyle \& C. Nemerhoff (Eds.), Neuropsychopharmacology: the fifth generation of progress. Philadelphia: Lippincott Williams and Wilkins.

Ahveninen, J., Kahkonen, S., Pennanen, S., Liesivuori, J., Ilmoniemi, R. J., \& Jaaskelainen, I. P. (2002). Tryptophan depletion effects on EEG and MEG responses suggest serotonergic modulation of auditory involuntary attention in humans. Neuroimage, 16(4), 10521061.

Alex, K. D., \& Pehek, E. A. (2006). Pharmacologic mechanisms of serotonergic regulation of dopamine neurotransmision. Pharmacology and Therapeutics, online.

Allain, H., Bentue Ferrer, D., Tribut, O., Merienne, M., \& Belliard, S. (2003). Drug therapy of frontotemporal dementia. Human psychopharmacology, 18(3), 221-225.

Amado-Boccara, I., Gougoulis, N., Poirier Littre, M. F., Galinowski, A., \& Loo, H. (1995). Effects of antidepressants on cognitive functions: a review. Neuroscience and Biobehavioral Reviews, 19(3), 479-493.

Anand, A., \& Charney, D. S. (2000). Norepinephrine dysfunction in depression. Journal of clinical psychiatry, 61 Suppl 10, 16-24.

Anderson, G. M., Barr, C. S., Lindell, S., Durham, A. C., Shifrovich, I., \& Higley, J. D. (2005). Time course of the effects of the serotonin-selective reuptake inhibitor sertraline on central and peripheral serotonin neurochemistry in the rhesus monkey. Psychopharmacology, 178(2-3), 339-346.

Anderson, I. M., Richell, R. A., \& Bradshaw, C. M. (2003). The effect of acute tryptophan depletion on probalistic choice. Journal of Psychopharmacology, 17(1), 3-7.

Anttila, S. A., \& Leinonen, E. V. (2001). A review of the pharmacological and clinical profile of mirtazapine. CNS drug reviews, 7(3), 249-264.

Aronson, S., \& Delgado, P. (2004). Escitalopram. Drugs of today Barcelona, Spain 1998, 40(2), 121131.

Auquier, P., Robitail, S., Llorca, P. M., \& Rive, B. (2003). Comparison of escitalopram and citalopram efficacy: A meta-analysis. International Journal of Psychiatry, 7(4), 259-268.

Austin, M. P., Mitchell, P., \& Goodwin, G. M. (2001). Cognitive deficits in depression: possible implications for functional neuropathology. British Journal of Psychiatry, 178, 200-206. 
Austin, M. P., Ross, M., Murray, C., O'Carroll, R. E., Ebmeier, K. P., \& Goodwin, G. M. (1992). Cognitive function in major depression. Journal of Affective Disorders, 25(1), 21-29.

Baddeley, A. D. (1968). A 3 Min Reasoning Test Based on Grammatical Transformation. Psychonomic Science, 10(10), 341-342.

Barbone, F., McMahon, A. D., Davey, P. G., Morris, A. D., Reid, I. C., McDevitt, D. G., et al. (1998). Association of road-traffic accidents with benzodiazepine use. Lancet, 352(9137), 1331-1336.

Beck, A. T., Ward, C. H., Mendelson, M., Mock, J., \& Erbaugh, J. (1961). An inventory for measuring depression. Archives of General Psychiatry, 4, 561-571.

Behrens, T. E., Johansen Berg, H., Woolrich, M. W., Smith, S. M., Wheeler Kingshott, C. A., Boulby, P. A., et al. (2003). Non-invasive mapping of connections between human thalamus and cortex using diffusion imaging. Nature neuroscience, 6(7), 750-757.

Bethea, C. L., Lu, N. Z., Gundlah, C., \& Streicher, J. M. (2002). Diverse actions of ovarian steroids in the serotonin neural system. Frontiers in Neuroendocrinology, 23, 41-100.

Biswas, P. N., Wilton, L. V., \& Shakir, S. A. (2003). The pharmacovigilance of mirtazapine: results of a prescription event monitoring study on 13554 patients in England. Journal of psychopharmacology Oxford, England, 17(1), 121-126.

Blier, P., Pineyro, G., el Mansari, M., Bergeron, R., \& de Montigny, C. (1998). Role of somatodendritic 5-HT autoreceptors in modulating 5-HT neurotransmission. Annals of the New York Academy of Sciences, 861, 204-216.

Blokland, A., Sik, A., \& Lieben, C. (2005). Evaluation of DOI, 8-OH-DPAT, eticlopride and amphetamine on impulsive responding in a reaction time task in rats. Behavioural pharmacology, 16(2), 93-100.

Bond, A., \& Lader, M. (1974). The use of analogue scales in rating subjective feelings. British Journal of Medical Psychology, 47(3), 211-218.

Bondareff, W., Alpert, M., Friedhoff, A. J., Richter, E. M., Clary, C. M., \& Batzar, E. (2000). Comparison of sertraline and nortriptyline in the treatment of major depressive disorder in late life. American Journal of Psychiatry, 157(5), 729-736.

Booij, L., van der Does, A. J. W., Haffmans, J. P. M., Riedel, W. J., Fekkes, D., \& Blom, M. J. B. (2005). The effects of high-dose and low-dose tryptophan depletion on mood and cognitive functions of remitted depressed patients. Journal of psychopharmacology, in press.

Booij, L., van der Does, A. J. W., \& Riedel, W. J. (2003). Monoamine depletion in psychiatric and healthy populations: review. Molecular Psychiatry, 8(951-973). 
Borkenstein, R. F., Crowther, R. F., Shumate, R. P., Ziel, W. B., \& Zylman, R. (1974). The role of the drinking driver in traffic accidents (The Grand Rapids study). Blutalkohol, 11(S1), 1-131.

Bortolozzi, A., Diaz Mataix, L., Scorza, M. C., Celada, P., \& Artigas, F. (2005). The activation of 5 -HT receptors in prefrontal cortex enhances dopaminergic activity. Journal of neurochemistry, 95(6), 1597-1607.

Brand, A. N., Jolles, J., \& Gispen de Wied, C. (1992). Recall and recognition memory deficits in depression. Journal of affective disorders, 25(1), 77-86.

Brookhuis, K. A., de Waard, D., \& Mulder, B. (1994). Measuring driving performance by carfollowing in traffic. Ergonomics, 37(3), 427-434.

Brunnauer, A., \& Laux, G. (2003). Fahrtuchtigkeit und Antidepressiva. Psychiatrische Praxis, 30 Suppl 2, S102-105.

Brunnauer, A., Laux, G., Geiger, E., Soyka, M., \& Moller, H. (2006). Antidepressants and driving ability: results from a clinical study. Journal of Clincal Psychiatry, 67, 1776-1781.

Buhot, M. (1997). Serotonin receptors in cognitive behaviors. Current Opinion in Neurobiology, $7(2), 243-254$.

Buhot, M. C., Martin, S., \& Segu, L. (2000). Role of serotonin in memory impairment. Annals of medicine, 32(3), 210-221.

Burt, D. B., Zembar, M. J., \& Niederehe, G. (1995). Depression and memory impairment: a meta-analysis of the association, its pattern, and specificity. Psychological bulletin, 117(2), 285-305.

Butters, M. A., Becker, J. T., Nebes, R. D., Zmuda, M. D., Mulsant, B. H., Pollock, B. G., et al. (2000). Changes in cognitive functioning following treatment of late-life depression. American journal of psychiatry, 157(12), 1949-1954.

Cabeza, R., \& Nyberg, L. (2000). Imaging cognition II: An empirical review of 275 PET and fMRI studies. Journal of cognitive neuroscience, 12(1), 1-47.

Camilleri, M. (2001). Management of the irritable bowel syndrome. Gastroenterology, 120(3), 652-668.

Capuron, L., Hauser, P., Hinze-Selch, D., Miller, A. H., \& Neveu, P. J. (2002). Treatment of cytokine-induced depression. Brain Behavior and Immunity, 16(5), 575-580.

Capuron, L., Neurauter, G., Musselman, D. L., Lawson, D. H., Nemeroff, C. B., Fuchs, D., et al. (2003). Interferon-alpha-induced changes in tryptophan metabolism. relationship to depression and paroxetine treatment. Biological Psychiatry, 54(9), 906-914.

Carli, M., \& Samanin, R. (2000). The 5-HT(1A) receptor agonist 8-OH-DPAT reduces rats' accuracy of attentional performance and enhances impulsive responding in a five- 
choice serial reaction time task: role of presynaptic 5-HT(1A) receptors. Psychopharmacology, 149(3), 259-268.

Catafau, A. M., Parellada, E., Lomena, F., Bernardo, M., Setoain, J., Catarineu, S., et al. (1998). Role of the cingulate gyrus during the Wisconsin Card Sorting Test: a single photon emission computed tomography study in normal volunteers. Psychiatry Research, 83(2), 67-74.

Chamberlain, S. R., Muller, U., T.W., R., \& B.J., S. (2006). Neuropharmacological modulation of cognition. Current Opinion in Neurology, 19(6), 607-612.

Charney, D. S. (1998). Monoamine dysfunction and the pathophysiology and treatment of depression. Journal of clinical psychiatry, 59 Suppl 14, 11-14.

Clark, L., Cools, R., \& Robbins, T. W. (2004). The neuropsychology of ventral prefrontal cortex: Decision-making and reversal learning. Brain and Cognition, 55, 41-53.

Clarke, H. F., Dalley, J. W., Crofts, H. S., Robbins, T. W., \& Roberts, A. C. (2004). Cognitive inflexibility after prefrontal serotonin depletion. Science, 304, 878-880.

Coffey, D. K., Jenkyn, L. R., Coffey, A. K., \& Wells, B. B. (1994). Sertraline vs amitriptyline vs placebo: effects on cognitive and motor functioning in the elderly. Neuropsychopharmacology, 10, 222S.

Conners, C. X. (1992). Conners' continuous performnace test computer program user's guide. Toronto: Multi-Health Systems.

Cools, R., Clark, L., Owen, A. M., \& Robbins, T. W. (2002). Defining the neural mechanisms of probalistic reversal learning using event-related functional magnetic resonance imaging. Journal of Neuroscience, 22, 4563-4567.

Corbetta, M., Kincade, J. M., \& Shulman, G. L. (2002). Neural systems for visual orienting and their relationships to spatial working memory. Journal of cognitive neuroscience, 14(3), 508-523.

Coull, J. T. (1998). Neural correlates of attention and arousal: insights from electrophysiology, functional neuroimaging and psychopharmacology. Progress in neurobiology, 55(4), 343-361.

Coull, J. T., Frackowiak, R. S., \& Frith, C. D. (1998). Monitoring for target objects: activation of right frontal and parietal cortices with increasing time on task. Neuropsychologia, 36(12), 1325-1334.

Coull, J. T., Sahakian, B. J., Middleton, H. C., Young, A. H., Park, S. B., McShane, R. H., et al. (1995). Differential effects of clonidine, haloperidol, diazepam and tryptophan depletion on focused attention and attentional search. Psychopharmacology, 121(2), 222230. 
Curran, H. V., \& Travill, R. A. (1997). Mood and cognitive effects of +/-3,4methylenedioxymethamphetamine (MDMA, 'ecstasy'): week-end 'high' followed by mid-week low. Addiction Abingdon, England, 92(7), 821-831.

Curran, H. V., \& Verheyden, S. L. (2003). Altered response to tryptophan supplementation after long-term abstention from MDMA (ecstasy) is highly correlated with human memory function. Psychopharmacology, 169(1), 91-103.

Curran, S. (1990). Critical Flicker Fusion techniques in psychopharmacology. In I. Hindmarch \& P. D. Stonier (Eds.), Human Psychopharmacology (Vol. 3). Chichester: John Wiley.

Curran, S., \& Wattis, J. P. (1998). Critical Flicker Fusion Threshold: A usefull research tool in patients with Alzheimers Disease. Human Psychopharmacology, 13, 337-355.

Danjou, P., Hamon, M., Lacomblez, L., \& Warot, D. (1990). Psychomotor, subjective and neuroendocrine effects of acute tryptophan depletion in the healthy volunteer. Psychiatrie et Psychobiologie, 5(1), 31-38.

Davis, K. L., Charney, D., Coyle, J. T., \& Nemerhoff, C. (Eds.). (2002). Neuropsychopharmacology: The Fifth Generation of Progress: American College of Neuropsychopharmacology.

De Boer, T., \& Ruigt, G. S. F. (1995). The selective a2-adrenoceptor antagonist mirtazapine (org 3770) enhances noradrenergic and 5-HT-1a-mediated serotonergic neurotransmission. CNS Drugs, 4(suppl1), 29-38.

de Quervain, D. J., Henke, K., Aerni, A., Coluccia, D., Wollmer, M. A., Hock, C., et al. (2003). A functional genetic variation of the 5-HT2a receptor affects human memory. Nature neuroscience, 6(11), 1141-1142.

de Wit, H., Crean, J., \& Richards, J. B. (2000). Effects of d-amphetamine and ethanol on a measure of behavioral inhibition in humans. Behavioral neuroscience, 114(4), 830-837.

de Wit, H., Enggasser, J. L., \& Richards, J. B. (2002). Acute administration of d-amphetamine decreases impulsivity in healthy volunteers. Neuropsychopharmacology, 27(5), 813-825.

Deakin JB, R. S., Nestor PJ, Hodges JR, Sahakian BJ. (2004). Paroxetin does improve symptoms and impairs cognition in frontotemporal dementia: a double-blind randomized controlled trial. Psychopharmacology, 172, 400-408.

Deijen, J. B., Loriaux, S. M., Orlebeke, J. F., \& De Vries, J. (1989). Effects of paroxetine and maprotiline on mood, perceptual-motor skills and eye movements in healthy volunteers. Journal of Psychopharmacology, 3(3), 148-155.

Del Ben, C. M., Deakin, J. F., McKie, S., Delvai, N. A., Williams, S. R., Elliott, R., et al. (2005). The effect of citalopram pretreatment on neuronal responses to neuropsychological tasks in normal volunteers: an FMRI study. Neuropsychopharmacology, 30(9), 1724-1734. 
Delagrange, P., Canu, M. H., Rougeul, A., Buser, P., \& Bouyer, J. J. (1993). Effects of locus coeruleus lesions on vigilance and attentive behaviour in cat. Behavioural Brain Research, 53(1-2), 155-165.

Den Hartog, H. M., Derix, M. M., Van Bemmel, A. L., Kremer, B., \& Jolles, J. (2003). Cognitive functioning in young and middle-aged unmedicated out-patients with major depression: testing the effort and cognitive speed hypotheses. Psychological medicine, 33(8), 1443-1451.

Di Matteo, V., De Blasi, A., Di Giulio, C., \& Esposito, E. (2001). Role of 5-HT(2C) receptors in the control of central dopamine function. Trends in pharmacological sciences, 22(5), 229232.

Doraiswamy, P. M., Krishnan, K. R., Oxman, T., Jenkyn, L. R., Coffey, D. J., Burt, T., et al. (2003). Does antidepressant therapy improve cognition in elderly depressed patients? Journals of gerontology, 58(12), M1137-1144.

Dornhoefer, S. M., Unema, P. J., \& Velichkovsky, B. M. (2002). Blinks, blanks and saccades: how blind we really are for relevant visual events. Progress in Brain Research, 140, 119-131.

Duman, R. S., Malberg, J., \& Nakagawa, S. (2001). Regulation of adult neurogenesis by psychotropic drugs and stress. Journal of Pharmacology and Experimental Therapeutics, 299(2), 401-407.

Dwivedi, Y., \& Pandey, G. N. (1998). Quantitation of 5HT2A receptor mRNA in human postmortem brain using competitive RT-PCR. Neuroreport, 9(17), 3761-3765.

Edgar, C. J., Preskorn, S., Erickson, M., Friesen, S., Werder, S., Waddington, G., et al. (2004). A Randomized, double-blind, parallel group comparison of the effects of escitalopram vs sertraline on cognition. Journal of Neuropsychopharmacology, 7(suppl 168).

Elliott, R., Baker, S. C., Rogers, R. D., O'Leary, D. A., Paykel, E. S., Frith, C. D., et al. (1997). Prefrontal dysfunction in depressed patients performing a complex planning task: a study using positron emission tomography. Psychological medicine, 27(4), 931-942.

Elliott, R., Sahakian, B. J., Herrod, J. J., Robbins, T. W., \& Paykel, E. S. (1997). Abnormal response to negative feedback in unipolar depression: evidence for a diagnosis specific impairment. Journal of Neurology Neurosurgery and Psychiatry, 63(1), 74-82.

Elliott, R., Sahakian, B. J., McKay, A. P., Herrod, J. J., Robbins, T. W., \& Paykel, E. S. (1996). Neuropsychological impairments in unipolar depression: the influence of perceived failure on subsequent performance. Psychological Medicine, 26(5), 975-989.

Elliott, R., Sahakian, B. J., Michael, A., Paykel, E. S., \& Dolan, R. J. (1997). Abnormal neural response to feedback on planning and guessing tasks in patients with unipolar depression. Psychological Medicine, 28, 559-571. 
Ellis, K. A., Mehta, M. A., Wesnes, K. A., Armstrong, S., \& Nathan, P. J. (2005). Combined D1/D2 receptor stimulation under conditions of dopamine depletion impairs spatial working memory performance in humans. Psychopharmacology, 181(4), 771-780.

Eriksen, B. A., \& Eriksen, C. W. (1974). Effects of noise letters upon the identification of a target letter in a nonsearch task. Perception and Psychophysics, 16(1), 143-149.

Esposito, E. (2006). Serotonin-dopamin interaction as a focus of novel antidepressant drugs. Current Drug Targets, 7, 177-185.

Evers, E. A., Tillie, D. E., van der Veen, F. M., Lieben, C. K., Jolles, J., Deutz, N. E., et al. (2005). Effects of a novel method of acute tryptophan depletion on plasma tryptophan and cognitive performance in healthy volunteers. Psychopharmacology, 178(1), 92-99.

Evers, E. A. T., Cools, R., Clark, L., van der Veen, F. M., Jolles, J., Sahakian, B. J., et al. (2005). Serotonergic modulation of prefrontal cortex during negative feedback in probabilistic reversal learning. Neuropsychopharmacology, 1-10.

Fadda, F. (2000). Tryptophan-Free Diets: A Physiological Tool to Study Brain Serotonin Function. News Physiol. Sci., 15, 260-264.

Fairweather, D. B., Ashford, J., \& Hindmarch, I. (1996). Effects of fluvoxamine and dothiepin on psychomotor abilities in healthy volunteers. Pharmacology Biochemistry and Behavior, 53(2), 265-269.

Fairweather, D. B., DalPozzo, C., Kerr, J. S., Lafferty, S. V., \& Hindmarch, I. (1997). Citalopram compared to dothiepin and placebo: Effects on cognitive function and psychomotor performance. Human Psychopharmacology Clinical and Experimental, 12(2), 119-126.

Fairweather, D. B., Kerr, J. S., Harrison, D. A., Moon, C. A., \& et al. (1993). A double blind comparison of the effects of fluoxetine and amitriptyline on cognitive function in elderly depressed patients. Human Psychopharmacology, 8(1), 41-47.

Finkel, S. I., Richter, E. M., \& Clary, C. M. (1999). Comparative efficacy and safety of sertraline versus nortriptyline in major depression in patients 70 and older. International psychogeriatrics IPA, 11(1), 85-99.

Finkel, S. I., Richter, E. M., Clary, C. M., \& Batzar, E. (1999). Comparative efficacy of sertraline vs. fluoxetine in patients age 70 or over with major depression. American journal of geriatric psychiatry official journal of the American Association for Geriatric Psychiatry, 7(3), 221-227.

Fossati, P., Ergis, A. M., \& Allilaire, J. F. (2002). Executive functioning in unipolar depression: a review. Encephale, 28(2), 97-107. 
Fox, H. C., McLean, A., Turner, J. J., Parrott, A. C., Rogers, R., \& Sahakian, B. J. (2002). Neuropsychological evidence of a relatively selective profile of temporal dysfunction in drug-free MDMA ("ecstasy") polydrug users. Psychopharmacology, 162(2), 203-214.

Furlan, P. M., Kallan, M. J., Ten Have, T., Pollock, B. G., Katz, I., \& Lucki, I. (2001). The cognitive and psychomotor effects of paroxetine and sertraline on healthy elderly subjects. American Journal of Geriatric Psychiatry, 9, 429-438.

Gallagher, P., Massey, A. E., Young, A. H., \& McAllister-Williams, R. H. (2003). Effects of acute tryptophan depletion on executive function in healthy male volunteers. BMCPsychiatry, 3(1), 10.

Garnett, E. S., Firnau, G., \& Nahmias, C. (1983). Dopamine visualized in the basal ganglia of living man. Nature, 305(5930), 137-138.

Genovese, C. R., Lazar, N. A., \& Nichols, T. (2002). Thresholding of statistical maps in functional neuroimaging using the false discovery rate. NeuroImage, 15(4), 870-878.

Gerhard, U., \& Hobi, V. (1984). Cognitive-Psychomotor Functions with Regard to Fitness for Driving of Psychiatric Patients Treated with Neuroleptics and Antidepressants. Neuropsychobiology, 12(1), 39-47.

Gottfries, C. G. (2001). Late life depression. European Archives of Psychiatry and Clinical Neuroscience, 251 Suppl 2, II57-61.

Gouzoulis-Mayfrank, E., Thimm, B., Rezk, M., Hensen, G., \& Daumann, J. (2003). Memory impairment suggests hippocampal dysfunction in abstinent ecstasy users. Prog Neuropsychopharmacol Biol Psychiatry, 27(5), 819-827.

Grabe, H. J., Wolf, T., Gratz, S., \& Laux, G. (1998). The influence of polypharmacological antidepressive treatment on central nervous information processing of depressed patients: implications for fitness to drive. Neuropsychobiology, 37(4), 200-204.

Grappe, H. J., Wolf, T., Gratz, S., \& Laux, G. (1998). The influence of polypharmacological antidepressive treatment on central nervous information processing of depressed patients: Implications for fitness to drive. Neuropsychobiology, 37(4), 200-204.

Green, A. R., Mechan, A. O., Elliott, J. M., O'Shea, E., \& Colado, M. I. (2003). The pharmacology and clinical pharmacology of 3,4-methylenedioxymethamphetamine (MDMA, "ecstasy"). Pharmacological reviews, 55(3), 463-508.

Hamilton, M. (1967). Development of a rating scale for primary depressive illness. British Journal of Social and Clinical Psychiatry, 16, 278-296.

Harmer, C. J., Bhagwagar, Z., Cowen, P. J., \& Goodwin, G. M. (2002). Acute administration of citalopram facilitates memory consolidation in healthy volunteers. Psychopharmacology (Berl), 163(1), 106-110. 
Harmer, C. J., McTavish, S. F. B., Clark, L., Goodwin, G. M., \& Cowen, P. J. (2001). Tyrosine depletion attenuates dopamine function in healthy volunteers. Psychopharmacology, 154, 105-111.

Harrison, A. A., Everitt, B. J., \& Robbins, T. W. (1997). Central 5-HT depletion enhances impulsive responding without affecting the accuracy of attentional performance: interactions with dopaminergic mechanisms. Psychopharmacology, 133(4), 329-342.

Harrison, B. J., Olver, J. S., Norman, T. R., Burrows, G. D., Wesnes, K. A., \& Nathan, P. J. (2004). Selective effects of acute serotonin and catecholamine depletion on memory in healthy women. Journal of Psychopharmacology, 18(1), 32-40.

Hedner, T., Persson, B., \& Berglund, G. (1983). Ketanserin, a novel 5-hydroxytryptamine antagonist: monotherapy in essential hypertension. British journal of clinical pharmacology, 16(2), 121-125.

Herberg, K. (2001). Verkehrs-und alltagssicherheit bei medikation mit citalopram. Psychopharmakotherapie, 18, 81-88.

Hindmarch, I. (1995). The behavioural toxicity of the selective serotonin reuptake inhibitors. International clinical psychopharmacology, 9 Suppl 4, 13-17.

Hindmarch, I. (1997). The effects of antidepressants on psychomotor function with particular reference to reboxetine. European neuropsychopharmacology the journal of the European College of Neuropsychopharmacology, 7 Suppl 1, S17-21; discussion S71-13.

Hindmarch, I., \& Harrison, C. (1988). The Effects of Paroxetine and other Antidepressants in Combination with Alcohol in Psychomotor Activity Related to Car Driving. Human Psychopharmacology, 3, 13-20.

Hindmarch, I., \& Shamsi, Z. (1999). Antihistamines: models to assess sedative properties, assessment of sedation, safety and other side-effects. Clinical and experimental allergy journal of the British Society for Allergy and Clinical Immunology, 29 Suppl 3, 133-142.

Hughes, J., Matrenza, C., Kemp, A., Harisson, B. J., Liley, D., \& Nathan, P. J. (2003). Selective effects of simultaneous monoamine depletion on mood and emotional responsiveness. International Journal of Neuropsychopharmacology, in press.

Hughes, J. H., Gallagher, P., Stewart, M. E., Matthews, D., Kelly, T. P., \& Young, A. H. (2003). The effects of acute tryptophan depletion on neuropsychological function. Journal of Psychopharmacology, 17(3), 300-309.

Jacobs, B. L., Praag, H., \& Gage, F. H. (2000). Adult brain neurogenesis and psychiatry: a novel theory of depression. Molecular Psychiatry, 5(3), 262-269.

Jex, H. R., McDonnell, J. D., \& Phatak, A. V. (1966). A "critical" tracking task for man-machine research related to the operators effective delay time.Part I: Theory and experiments 
with a first order divergent controlled element. NASA CR-616, NASA Contractor Report. NASA CR, 100-105.

Kapur, S., \& Remington, G. (1996). Serotonin-dopamine interaction and its relevance to schizophrenia. American Journal of Psychiatry, 153(4), 466-476.

Kasper, S., Praschak Rieder, N., Tauscher, J., \& Wolf, R. (1997). A risk-benefit assessment of mirtazapine in the treatment of depression. Drug safety an international journal of medical toxicology and drug experience, 17(4), 251-264.

Kerr, J. S., Fairweather, D. B., Mahendran, R., \& Hindmarch, I. (1992). The effects of paroxetine, alone and in combination with alcohol on psychomotor performance and cognitive function in the elderly. International Journal of Psychopharmacology, 7(2), 101-108.

Kerr, J. S., Sherwood, N., \& Hindmarch, I. (1991). The comparative psychopharmacology of 5HT reuptake inhibitors. Human Psychopharmacology Clinical and Experimental, 6(4), 313-317.

Kilkens, T. O., Honig, A., Rozendaal, N., Van Nieuwenhoven, M. A., \& Brummer, R. J. (2003). Serotonergic modulators in the treatment of irritable bowel syndrome - influence on psychiatric and gastrointestinal symptoms. Alimentary Pharmacology $\mathcal{E}$ Therapeutics, 17(1), 43-51.

Kilkens, T. O., Honig, A., van Nieuwenhoven, M. A., Riedel, W. J., \& Brummer, R. J. (2004). Acute tryptophan depletion affects brain-gut responses in irritable bowel syndrome patients and controls. Gut, 53(12), 1794-1800.

Kimura, D., \& D'Amico, C. (1989). Evidence for subgroups of adextrals based on speech lateralization and cognitive patterns. Neuropsychologia, 27(7), 977-986.

Klaassen, T., Riedel, W. J., Deutz, N. E., \& Van Praag, H. M. (2002). Mood congruent memory bias induced by tryptophan depletion. Psychological medicine, 32(1), 167-172.

Klaassen, T., Riedel, W. J., Deutz, N. E. P., Van Someren, A., \& Van Praag, H. M. (1999). Specificity of the tryptophan depletion method. Psychopharmacology, 141(3), 279-286.

Koelega, H. S. (1993). Stimulant drugs and vigilance performance: a review. Psychopharmacology, 111(1), 1-16.

Koetsier, G. C., Volkers, A. C., Tulen, J. H., Passchier, J., van den Broek, W. W., \& Bruijn, J. A. (2002). CPT performance in major depressive disorder before and after treatment with imipramine or fluvoxamine. Journal of psychiatric research, 36(6), 391-397.

Krawczyk, D. C. (2002). Contributions of the prefrontal cortex to the neural basis of human decision making. Neuroscience and Biobehavioral Reviews, 26(6), 631-664.

Kringelbach, M. L., \& Rolls, E. T. (2003). Neural correlates of rapid reversal learning in a simple model of human social interaction. NeuroImage, 20(2), 1371-1383. 
Kronfol, Z. (2002). Immune dysregulation in major depression: a critical review of existing evidence. International Journal of Neuropsychopharmacology, 5(4), 333-343.

Kuypers, K. P. C., \& Ramaekers, J. G. (2005). Transient memory impairment after acute dose of 75mg 3.4-Methylene-dioxymethamphetamine. Journal of psychopharmacology Oxford, England, 19(6), 633-639.

Kuypers, K. P. C., \& Ramaekers, J. G. (2006). Acute dose of MDMA (75 mg) impairs spatial memory for location but leaves contextual processing of visuospatial information unaffected. Psychopharmacology, DOI 10.1007/s00313-006-0321-7.

La Berge, D. (1995). Attentional procesing. Cambridge: Harvard UP.

La Pia, S., Fuschillo, C., Giorgio, D., Ciriello, R., Pinto, A., Rivellini, M., et al. (2001). Sertonin (5HT)-related symptoms and fluoxetine in geriatric depression. Archives of gerontology and geriatrics, 33 Suppl 1, 213-225.

Lai, M. K. P., Tsang, S. W. Y., Francis, P. T., Keene, J., Hope, T., Esiri, M. M., et al. (2002). Postmortem serotonergic correlated of cognitive decline in Alzheimer's disease. Neuroreport, 13(9), 1175-1178.

Lamers, C. T. J., \& Ramaekers, J. G. (2001). Visual search and urban city driving under the influence of marijuana and alcohol. Human Psychopharmacology: Clinical and Experimental, 16(5), 393-401.

Landro, N. I., Stiles, T. C., \& Sletvold, H. (2001). Neuropsychological function in nonpsychotic unipolar major depression. Neuropsychiatry, neuropsychology, and behavioral neurology, 14(4), 233-240.

Lane, R. M., \& O'Hanlon, J. F. (1999). Cognitive and psychomotor effects of antidepresants with emphasis on selective serotonin reuptake inhiitors and the depressed elderly patient. German Journal of Psychiatry, 2, 1-28.

Lawrence, N. S., Ross, T. J., Hoffmann, R., Garavan, H., \& Stein, E. A. (2003). Multiple neuronal networks mediate sustained attention. Journal of cognitive neuroscience, 15(7), 10281038.

Lembo, T. (2000). Neurotransmitter antagonism in management of irritable bowel syndrome. Lancet, 355(9209), 1030-1031.

Lepola, U. M., Loft, H., \& Reines, E. H. (2003). Escitalopram (10-20 mg/day) is effective and well tolerated in a placebo-controlled study in depression in primary care. International clinical psychopharmacology, 18(4), 211-217.

Leveille, S. G., Buchner, D. M., Koepsell, T. D., McCloskey, L. W., Wolf, M. E., \& Wagner, E. H. (1994). Psychoactive medications and injurious motor vehicle collisions involving older drivers. Epidemiology, 5(6), 591-598. 
Levkovitz, Y., Caftori, R., Avital, A., \& Richter Levin, G. (2002). The SSRIs drug Fluoxetine, but not the noradrenergic tricyclic drug Desipramine, improves memory performance during acute major depression. Brain research bulletin, 58(4), 345-350.

Lewin, J. S., Friedman, L., Wu, D., Miller, D. A., Thompson, L. A., Klein, S. K., et al. (1996). Cortical localization of human sustained attention: detection with functional MR using a visual vigilance paradigm. Journal of computer assisted tomography, 20(5), 695701.

Lidow, M. S., Koh, P. O., \& Arnsten, A. F. (2003). D1 dopamine receptors in the mouse prefrontal cortex: Immunocytochemical and cognitive neuropharmacological analyses. Synapse New York, N.Y., 47(2), 101-108.

Liechti, M. E., Saur, M. R., Gamma, A., Hell, D., \& Vollenweider, F. X. (2000). Psychological and physiological effects of MDMA ("Ecstasy") after pretreatment with the 5-HT(2) antagonist ketanserin in healthy humans. Neuropsychopharmacology, 23(4), 396-404.

Logan, G. D. (1994). On the ability to inhibit thought and action. In D. Dagenbach \& T. H. Carr (Eds.), Inhibitory processes in attentin, memory and language. San Diego: Academic Press.

Louwerens, J. W., Gloerich, A. B. M., De Vries, G., Brookhuis, K. A., \& O'Hanlon, J. F. (1987). The relationship between drivers' blood alcohol concentration (BAC) and actual driving performance during high speed travel. In P. C. Noordzij \& R. Roszbach (Eds.), Alcohol, drugs and traffic safety (pp. 183-186). Amsterdam: Elsevier.

Lucas, G., \& Spampinato, U. (2000). Role of striatal serotonin2A and serotonin2C receptor subtypes in the control of in vivo dopamine outflow in the rat striatum. Journal of neurochemistry, 74(2), 693-701.

Luciana, M., Collins, P. F., \& Depue, R. A. (1998). Opposing roles for dopamine and serotonin in the modulation of human spatial working memory functions. Cereb Cortex, 8(3), 218-226.

Mackworth, N. H. (1950). Researches on the measurement of human performance. In H. W. Sinaiko (Ed.), Selected papers on human factors in the design and use of control systems (pp. 174-331). New York: Dover.

Maes, M., \& Meltzer, H. Y. (1995). The Serotonin Hypothesis of Major Depression. In F. E. Bloom \& D. J. Kupfer (Eds.), Psychopharmacology: The Fourth Generation of Progress (pp. 933-944). New York: Raven Press.

Matrenza, C., Hughes, J. M., Kemp, A. H., Wesnes, K. A., Harrison, B. J., \& Nathan, P. J. (2004). Simultaneous depletion of serotonin and catecholamines impairs sustained attention in healthy female subjects without affecting learning and memory. Journal of psychopharmacology, 18(1), 21-31. 
Mattila, M., Mattila, M. J., Vrijmoed de Vries, M., \& Kuitunen, T. (1989). Actions and interactions of psychotropic drugs on human performance and mood: single doses of ORG 3770, amitriptyline, and diazepam. Pharmacology and toxicology, 65(2), 81-88.

Mattila, M. J., Saarialho-Kere, U., \& Mattila, M. (1988). Acute effects of sertraline, amitriptyline, and placebo on the psychomotor performance fo healthy subjects over 50 years of age. J Clin Psychiatry, 49(S8), 52-58.

McAllister-Williams, R. H., Massey, A. E., \& Rugg, M. D. (2002). Effects of tryptophan depletion on brain potential correlates of episodic memory retrieval. Psychopharmacology, 160(4), 434-442.

McCann, U. D., Eligulashvili, V., \& Ricaurte, G. A. (2000). (+/-)3,4Methylenedioxymethamphetamine ('Ecstasy')-Induced Serotonin Neurotoxicity: Clinical Studies. Neuropsychobiology, 42(1), 11-16.

McCann, U. D., Szabo, Z., Scheffel, U., Dannals, R. F., \& Ricaurte, G. A. (1998). Positron emission tomographic evidence of toxic effect of MDMA ("Ecstasy") on brain serotonin neurons in human beings. Lancet, 352(9138), 1433-1437.

McGaughy, J., Sandstrom, M., Ruland, S., Bruno, J. P., \& Sarter, M. (1997). Lack of effects of lesions of the dorsal noradrenergic bundle on behavioral vigilance. Behavioral Neuroscience, 111(3), 646-652.

McGuirk, J., \& Silverstone, T. (1990). The effect of the 5-HT re-uptake inhibitor fluoxetine on food intake and body weight in healthy male subjects. International Journal of Obesity and Related Metabolic Disorders, 14(4), 361-372.

McKeown, M. J., \& Sejnowski, T. J. (1998). Independent component analysis of fMRI data: examining the assumptions. Human brain mapping, 6(5-6), 368-372.

McKie, S., Del Ben, C., Elliott, R., Williams, S., del Vai, N., Anderson, I., et al. (2005). Neuronal effects of acute citalopram detected by pharmacoMRI. Psychopharmacology, 180(4), 680686.

McNair, D. M., Lorr, M., \& Droppleman, L. F. (1992). Manual for the Profile of Mood States (Revised). San Diego: Educational and Industrial Testing Service.

Meeter, M., Talamini, L., Schmitt, J. A., \& Riedel, W. J. (2006). Effects of 5-HT on memory and the hippocampus: model and data. Neuropsychopharmacology, 31(4), 712-720.

Meltzer, C. C., Smith, G., DeKosky, S. T., Pollock, B. G., Mathis, C. A., Moore, R. Y., et al. (1998). Serotonin in aging, late-life depression and Alzheimer's disease: The emerging role of functional imaging. Neuropsychopharmacology, 18(6), 407-430.

Meltzer, H. (1989). Serotonergic dysfunction in depression. British Journal of Psychiatry, 8 (suppl), 25-31. 
Meneses, A. (1999). 5-HT system and cognition. Neuroscience and biobehavioral reviews, 23(8), 1111-1125.

Meneses, A. (2002). Involvement of 5-HT(2A/2B/2C) receptors on memory formation: simple agonism, antagonism, or inverse agonism? Cellular and molecular neurobiology, 22(5-6), 675-688.

Micale, V., Marco Leggio, G., Mazzola, C., \& Drago, F. (2006). Cognitive effects of SL65.0155, a serotonin 5-HT(4) receptor partial agonist, in animal models of amnesia. Brain Res, 22(1), 207-215.

Montgomery, S. A. (1995). Safety of mirtazapine: a review. International clinical psychopharmacology, 10 Suppl 4, 37-45.

Moore, P., Landolt, H. P., Seifritz, E., Clark, C., Bhatti, T., Kelsoe, J., et al. (2000). Clinical and physiological consequences of rapid tryptophan depletion. Neuropsychopharmacology, 23(6), 601-622.

Moretti, R., Torre, P., Antonello, R. M., Cazzato, G., \& Bava, A. (2002). Depression and Alzheimer's disease: symptom or comorbidity? American Journal of Alzheimer's Disease and Other Dementias, 17(6), 338-344.

Moskowitz, H. (1973). Laboratory studies of the efects of alcohol on some variables related to driving. Journal of Safety Research, 5, 185-192.

Mottaghy, F. M., Willmes, K., Horwitz, B., Muller, H. W., Krause, B. J., \& Sturm, W. (2006). Systems level modeling of a neuronal network subserving intrinsic alertness. NeuroImage, 29(1), 225-233.

Mulder-Hajonides van der Meulen, W. R. E. H. (1981). Measurement of subjective sleep quality. Proceedings of the International European Sleep Congress. Amsterdam: Elsevier.

Murphy F.C., Rubinsztein J.S., Michael A., Robbins T.W., Paykel E.S., \& B.J., S. (2001). Decision-making cognition in mania and depression. Psychological Medicine, 31(4), 679693.

Murphy, F. C., Michael, A., Robbins, T. W., \& Sahakian, B. J. (2003). Neuropsychological impairment in patients with major depressive disorder: the effects of feedback on task performance. Psychological Medicine, 33(3), 455-467.

Murphy, F. C., Smith, K. A., Cowen, P. J., Robbins, T. W., \& Sahakian, B. J. (2002). The effects of tryptophan depletion on cognitive and affective processing in healthy volunteers. Psychopharmacology, 163, 42-53.

Nakai, A., Kumakura, Y., Boivin, M., Rosa, P., Diksic, M., D'Souza, D., et al. (2003). Sex differences of brain serotonin synthesis in patients with irritable bowel syndrome 
using alpha-[11C]methyl-L-tryptophan, positron emission tomography and statistical parametric mapping. Canadian Journal of Gastroenterology, 17(3), 191-196.

Nathan, P. J., Sitaram, G., Stough, C., Silberstein, R. B., \& Sali, A. (2000). Serotonin, noradrenaline and cognitive function: a preliminary investigation of the acute pharmacodynamic effects of a serotonin versus a serotonin and noradrenaline reuptake inhibitor. Behavioural pharmacology, 11(7-8), 639-642.

Nebes, R. D., Pollock, B. G., Houck, P. R., Butters, M. A., Mulsant, B. H., Zmuda, M. D., et al. (2003). Persistence of cognitive impairment in geriatric patients following antidepressant treatment: a randomized, double-blind clinical trial with nortriptyline and paroxetine. Journal of psychiatric research, 37(2), 99-108.

Newhouse, P., Tatro, A., Naylor, M., Quealey, K., \& Delgado, P. L. (2002). Alzheimer Disease, serotonin systems and tryptophan depletion. American Journal of Geriatric Psychiatry, 10(4), 483-484.

Newhouse, P. A., Krishnan, K. R., Doraiswamy, P. M., Richter, E. M., Batzar, E. D., \& Clary, C. M. (2000). A double-blind comparison of sertraline and fluoxetine in depressed elderly outpatients. Journal of clinical psychiatry, 61(8), 559-568.

Nicholson, A. N., \& Pascoe, P. A. (1990). Dopaminergic transmission and the sleep-wakefulness continuum in man. Neuropharmacology, 29(4), 411-417.

Nishizawa, S., Benkelfat, C., Young, S. N., Leyton, M., Mzengeza, S., de Montigny, C., et al. (1997). Differences between males and females in rates of serotonin synthesis in human brain. Proceedings of the National Acadamy of Science, 94(10), 5308-5313.

Nutt, D. J. (2002). Tolerability and safety aspects of mirtazapine. Human psychopharmacology, 17 Suppl 1, S37-41.

O'Brien, S. M., Scott, L. V., \& Dinan, T. G. (2004). Cytokines: abnormalities in major depression and implications for pharmacological treatment. Human psychopharmacology, 19(6), 397-403.

O'Conner, C., Manly, T., Robertson, I. H., Hevenor, S. J., \& Levine, B. (2004). An fMRI study of sustained attention with endogenous and exogenous engagement. Brain and Cognition, 54, 133-176.

O'Hanlon, J., Robbe, H. W. J., Vermeeren, A., VanLeeuwen, C., \& Danjou, P. E. (1998). Venlafaxine's effects on healthy volunteers' driving, psychomotor, and vigilance performance during 15-day fixed and incremental dosing regimens. Journal of Clinical Psychopharmacology, 18(3), 212-221.

O'Hanlon, J. F. (1984). Driving performance under the influence of drugs: rationale for, and application of, a new test. British Journal of Pharmacology, 18 Suppl 1, 121S-129S. 
O'Hanlon, J. F., \& Ramaekers, J. G. (1995). Antihistamine effects on actual driving performance in a standard test: a summary of Dutch experience, 1989-94. Allergy, 50(3), 234-242.

Ordway, G. A., Klimek, V., \& Mann, J. J. (2002). Neurocircuitry of mood disorders. In K. L. Davis, D. Charney, J. T. Coyle \& C. Nemeroff (Eds.), Neuropsychopharmacology the fifth generation of progress: American College of Neuropsychopharmacology.

Owens, M. J., Knight, D. L., \& Nemeroff, C. B. (2001). Second-generation SSRIs: human monoamine transporter binding profile of escitalopram and R-fluoxetine. Biological psychiatry, 50(5), 345-350.

Park, S. B., Coull, J. T., McShane, R. H., Young, A. H., Sahakian, B. J., Robbins, T. W., et al. (1994). Tryptophan Depletion in Normal Volunteers Produces Selective Impairments in Learning and Memory. Neuropharmacology, 33(3/4), 575-588.

Parrott, A. C., Lees, A., Garnham, N. J., Jones, M., \& Wesnes, K. (1998). Cognitive performance in recreational users of MDMA of 'ecstasy': evidence for memory deficits. Journal of psychopharmacology, 12(1), 79-83.

Portas, C. M., Rees, G., Howseman, A. M., Josephs, O., Turner, R., \& Frith, C. D. (1998). A specific role for the thalamus in mediating the interaction of attention and arousal in humans. Journal of neuroscience, 18(21), 8979-8989.

Porter, R. J., Gallagher, P., Watson, S., \& Young, A. H. (2004). Corticosteroid-serotonin interactions in depression: a review of the human evidence. Psychopharmacology, 173, $1-17$.

Porter, R. J., Lunn, B. S., \& O'Brien, J. T. (2003). Effects of acute tryptophan depletion on cognitive function in Alzheimer's Disease and the healthy ederly. Psychological Medicine, 33, 41-49.

Porter, R. J., Lunn, B. S., Walker, L. L., Gray, J. M., Ballard, C. G., \& O'Brien, J. T. (2000). Cognitive deficit induced by acute tryptophan depletion in patients with Alzheimer's disease. American Journal of Psychiatry, 157(4), 638-640.

Pribram, K. H., \& McGuinness, D. (1975). Arousal, activation, and effort in the control of attention. Psychological review, 82(2), 116-149.

Purcell, R., Maruff, P., Kyrios, M., \& Pantelis, C. (1997). Neuropsychological function in young patients with unipolar major depression. Psychological Medicine, 27(6), 1277-1285.

Rabiner, E. A., Bhagwagar, Z., Gunn, R. N., Cowen, P. J., \& Grasby, P. M. (2004). Preferential 5HT1A autoreceptor occupancy by pindolol is attenuated in depressed patients: effect of treatment or an endophenotype of depression? Neuropsychopharmacology, 29(9), 1688-1698. 
Rabiner, E. A., Bhagwagar, Z., Gunn, R. N., Sargent, P. A., Bench, C. J., Cowen, P. J., et al. (2001). Pindolol augmentation of selective serotonin reuptake inhibitors: PET evidence that the dose used in clinical trials is too low. American journal of psychiatry, 158(12), 2080-2082.

Rabiner, E. A., Gunn, R. N., Wilkins, M. R., Sargent, P. A., Mocaer, E., Sedman, E., et al. (2000). Drug action at the 5-HT(1A) receptor in vivo: autoreceptor and postsynaptic receptor occupancy examined with PET and [carbonyl-11C] WAY-100635. Nuclear medicine and biology, 27(5), 509-513.

Radhakishun, F. S., van den Bos, J., van der Heijden, B. C., Roes, K. C., \& O'Hanlon, J. F. (2000). Mirtazapine effects on alertness and sleep in patients as recorded by interactive telecommunication during treatment with different dosing regimens. Journal of clinical psychopharmacology, 20(5), 531-537.

Rahman, S., Sahakian, B. J., Hodges, J. R., Rogers, R. D., \& Robbins, T. W. (1999). Specific cognitive deficits in mild frontal variant frontotemporal dementia. Brain, 122(Pt 8), 1469-1493.

Raichle, M. E., MacLeod, A. M., Snyder, A. Z., Powers, W. J., Gusnard, D. A., \& Shulman, G. L. (2001). A default mode of brain function. Proceedings of the National Academy of Sciences of the United States of America, 98(2), 676-682.

Ramaekers, G., Lamers, J., Verhey, F., Muntjewerff, D., Mobbs, E., Sanders, N., et al. (2002). A comparative study of the effects of carbamazepine and the NMDA receptor antagonist remacemide on road tracking and car-following performance in actual traffic. Psychopharmacology (Berl), 159(2), 203-210.

Ramaekers, J. G. (1998). Behavioural toxicity of medicinal drugs. Practical consequences, incidence, management and avoidance. Drug safety an international journal of medical toxicology and drug experience, 18(3), 189-208.

Ramaekers, J. G. (2003). Antidepressants and driver impairment: empirical evidence from a standard on-the-road test. Journal of Clinical Psychiatry, 64(1), 20-29.

Ramaekers, J. G., Ansseau, M., Muntjewerff, N. D., Sweens, J. P., \& O'Hanlon, J. F. (1997). Considering the P450 cytochrome system as determining combined effects of antidepressants and benzodiazepines on actual driving performance of depressed outpatients. International Clinical Psychopharmacology, 12(3), 159-169.

Ramaekers, J. G., \& Kuypers, K. P. C. (2006). Acute effects of 3,4methylenedioxymethamphetamine (MDMA) on behavioral measures of impulsivity: alone and in combination with alcohol. Neuropsychopharmacology, 31(5), 1048-1055. 
Ramaekers, J. G., Muntjewerff, N. D., \& O'Hanlon, J. F. (1995). A comparative study of acute and subchronic effects of dothiepin, fluoxetine and placebo on psychomotor and actual driving performance. British Journal of Pharmacology, 39(4), 397-404.

Ramaekers, J. G., Muntjewerff, N. D., Van Veggel, L. M. A., Uiterwijk, M. M. C., \& O'Hanlon, J. F. (1998). Effects of nocturnal doses of mirtazapine and mianserin on sleep and on daytime psychomotor and driving performance in young, healthy volunteers. Human Psychopharmacology, 13(Suppl 2), S87-S97.

Ramasubbu, R. (2004). Cerebrovascular effects of selective serotonin reuptake inhibitors: a systematic review. Journal of clinical psychiatry, 65(12), 1642-1653.

Raptopoulos, P., McClelland, G. R., \& Jackson, D. (1989). The clinical pharmacology of paroxetine in healthy subjects. Acta psychiatrica Scandinavica. Supplementum, 350, 46-48.

Rasmussen, K., McCreary, A. C., \& Shanks, E. A. (2004). Attenuation of the effects of fluoxetine on serotonergic neuronal activity by pindolol in rats. Neuroscience letters, 355(1-2), 1-4.

Ray, W. A., Fought, R. L., \& Decker, M. D. (1992). Psychoactive drugs and the risk of injurious motor vehicle crashes in elderly drivers. American journal of epidemiology, 136(7), 873883.

Reilly, J. G., McTavish, S. F. B., \& Young, A. H. (1997). Rapid depletion of plasma tryptophan: a review of studies and experimental methodology. Journal Of Psychopharmacology, 11(4), 381-392.

Reneman, L. (2001). Investigating the potential neurotoxicity of Ecstasy (MDMA); an imaging approach. Universiteit van Amsterdam, Amsterdam.

Rey, A. (1964). L'examen psychologique dans les cas d'encéphalopathie traumatique. Paris: Presses Universitaires de France.

Rezai, K., Andreasen, N. C., Alliger, R., Cohen, G., Swayze, V., \& O'Leary, D. S. (1993). The neuropsychology of the prefrontal cortex. Archives of Neurology, 50(6), 636-642.

Ricaurte, G. A., Yuan, J., \& McCann, U. D. (2000). (+/-)3,4-Methylenedioxymethamphetamine ('Ecstasy')-Induced Serotonin Neurotoxicity: Studies in Animals. Neuropsychobiology, 42(1), 5-10.

Richardson, J. S., Keegan, D. L., Bowen, R. C., Blackshaw, S. L., Cebrian Perez, S., Dayal, N., et al. (1994). Verbal learning by major depressive disorder patients during treatment with fluoxetine or amitriptyline. International clinical psychopharmacology, 9(1), 35-40.

Richer, F., Martinez, M., Cohen, H., \& Saint Hilaire, J. M. (1991). Visual motion perception from stimulation of the human medial parieto-occipital cortex. Experimental brain research, 87(3), 649-652. 
Richter-Levin, G., \& Segal, M. (1996). Serotonin, Aging and Cognitive Functions of the Hippocampus. Reviews in the Neurosciences, 7, 103-113.

Ridout, F., Meadows, R., Johnsen, S., \& Hindmarch, I. (2003). A placebo controlled investigation into the effects of paroxetine and mirtazapine on measures related to car driving performance. Human psychopharmacology, 18(4), 261-269.

Riedel, W. J., Eikmans, K., Heldens, A., \& Schmitt, J. A. (2005). Specific serotonergic reuptake inhibition impairs vigilance performance acutely and after subchronic treatment. Journal of psychopharmacology, 19(1), 12-20.

Riedel, W. J., Klaassen, T., Deutz, N. E., van Someren, A., \& van Praag, H. M. (1999). Tryptophan depletion in normal volunteers produces selective impairment in memory consolidation. Psychopharmacology (Berl), 141(4), 362-369.

Riedel, W. J., Klaassen, T., Griez, E., Honig, A., Menheere, P. P., \& van Praag, H. M. (2002). Dissociable hormonal, cognitive and mood responses to neuroendocrine challenge: evidence for receptor-specific serotonergic dysregulation in depressed mood. Neuropsychopharmacology, 26(3), 358-367.

Riedel, W. J., Klaassen, T., \& Schmitt, J. A. (2002). Tryptophan, mood, and cognitive function. Brain, behavior, and immunity, 16(5), 581-589.

Riedel, W. J., Schoenmakers, E., Vermeeren, A., \& O'Hanlon, J. F. (1999). The influence of trazodone treatment on cognitive functions in outpatients with major depressive disorder. 14(7), 499-508.

Robbins, T. W. (1997). Arousal systems and attentional processes. Biological psychology, 45(1-3), 57-71.

Robbins, T. W. (2000). Chemical neuromodulation of frontal-executive functions in humans and other animals. Exp Brain Res, 133(1), 130-138.

Robbins, T. W., Granon, S., Muir, J. L., Durantou, F., Harrison, A., \& Everitt, B. J. (1998). Neural systems underlying arousal and attention. Implications for drug abuse. Annals of the New York Academy of Sciences, 846, 222-237.

Rogers, R. D., Blackhaw, A. J., Middleton, H. C., Matthews, K., Hawtin, H., Crowley, C., et al. (1999). Tryptophan depletion impairs stimulus-reward learning while methylphenidate disrupts attentional control in healthy young adults: implications for the monoaminergic basis of impulsive behaviour. Psycopharmacology, 146, 482-491.

Rogers, R. D., Everitt, B. J., Baldacchino, A., Blackshaw, A. J., Swainson, R., Wynne, K., et al. (1999). Dissociable deficits in the decision-making cognition of chronic amphetamine abusers, opiate abusers, patients with focal damage to prefrontal cortex, and tryptophan-depleted normal volunteers. Neuropsychopharmacology, 20(4), 322-339. 
Rogers, R. D., Owen, A. M., Middleton, H. C., Williams, E. J., Pickard, J. D., Sahakian, B. J., et al. (1999). Choosing between small, likely rewards and large, unlikely rewards activates inferior and orbital prefrontal cortex. Journal of Neuroscience, 19(20), 9029-9038.

Rogers, R. D., Tunbridge, E. M., Bhagwagar, Z., Drevets, W. C., Sahakian, B. J., \& Carter, C. S. (2003). Tryptophan depletion alters decision-making of healthy volunteers through altered processing of reward cues. Neuropsychopharmacology, 28(1), 153-162.

Roth, B. L., Hanizavareh, S. M., \& Blum, A. E. (2004). Serotonin receptors represent highly favorable molecular targets for cognitive enhancement in schizophrenia and other disorders. Psychopharmacology, 174(1), 17-24.

Rowley, B., Van, F., Mortimore, C., \& Connell, J. (1998). Effects of Acute Tryptophan Depletion on Tests of Frontal and Temporal Lobe Function. Journal of Psychopharmacology, 12(3), A60.

Rubinsztein, J., Rogers, R. D., Riedel, W. J., Mehta, M. A., Robbins, T. W., \& Sahakian, B. J. (2001). Acute dietary tryptophan depletion impairs affective shifting and delayed visual recognition in healthy volunteers. Psychopharmacology, 154(3), 319-326.

Russo, S., Nielen, M. M., Boon, J. C., Kema, I. P., Willemse, P. H., de Vries, E. G., et al. (2003). Neuropsychological investigation into the carcinoid syndrome. Psychopharmacology, 168(3), 324-328.

Sabbe, B., van Hoof, J., Hulstijn, W., \& Zitman, F. (1996). Changes in fine motor retardation in depressed patients treated with fluoxetine. Journal of affective disorders, 40(3), 149-157.

Sagberg, F. (2006). Driver health and crash involvement: a case-control study. Accident analysis and prevention, 38(1), 28-34.

Saletu, B., \& Grunberger, J. (1988). Drug profiling by computed electroencephalography and brain maps, with special consideration of sertraline and its psychometric effects. Journal of clinical psychiatry, 49 Suppl, 59-71.

Sanchez, C., Bogeso, K. P., Ebert, B., Reines, E. H., \& Braestrup, C. (2004). Escitalopram versus citalopram: the surprising role of the R-enantiomer. Psychopharmacology, 174(2), 163176.

Schatzberg, A. F., Garlow, S. J., \& Nemeroff, C. B. (2002). Molecular And Cellular Mechanisms In Depression. In K. L. Davis, D. Charney, J. T. Coyle \& C. Nemerhoff (Eds.), Neuropsychopharmacology: The Fifth Generation of Progress: American College of Neuropsychopharmacology.

Schmitt, J. A., Jorissen, B. L., Sobczak, S., van Boxtel, M. P., Hogervorst, E., Deutz, N. E., et al. (2000). Tryptophan depletion impairs memory consolidation but improves focussed attention in healthy young volunteers. Journal of psychopharmacology, 14(1), 21-29. 
Schmitt, J. A., Kruizinga, M. J., \& Riedel, W. J. (2001). Non-serotonergic pharmacological profiles and associated cognitive effects of serotonin reuptake inhibitors. Journal of Psychopharmacology, 15(3), 173-179.

Schmitt, J. A., Riedel, W. J., Vuurman, E. F., Kruizinga, M., \& Ramaekers, J. G. (2002). Modulation of the critical flicker fusion effects of serotonin reuptake inhibitors by concomitant pupillary changes. Psychopharmacology (Berl), 160(4), 381-386.

Schmitt, J. A. J. (2001). Serotonin, caffeine and cognition: psychopharmacological studies in human cognitive functioning. Maastricht: Neuropsych Publishers.

Schmitt, J. A. J., Ramaekers, J. G., Kruizinga, M. J., van Boxtel, M. P., Vuurman, E. F., \& Riedel, W. J. (2002). Additional dopamine reuptake inhibition attenuates vigilance impairment induced by serotonin reuptake inhibition in man. Journal of Psychopharmacology, 16(3), 207-214.

Schmitt, J. A. J., Wingen, M., Ramaekers, J. G., Evers, E. A. T., \& Riedel, W. J. (2006). Serotonin and human cognitive performance. Current Pharmaceutical Design, 12, 2473-2486.

Schreiber, R., Vivian, J., Hedley, L., Szczepanski, K., Secchi, R. L., Zuzow, M., et al. (2006). Effects of the novel 5-HT6 receptor antagonist RO4368554 in rat models for cognition and sensorimotor gating. European Neuropsychopharmacology, online.

Seifritz, E., Baumann, P., Muller, M. J., Annen, O., Amey, M., Hemmeter, U., et al. (1996). Neuroendocrine effects of a $20-\mathrm{mg}$ citalopram infusion in healthy males. A placebocontrolled evaluation of citalopram as 5-HT function probe. Neuropsychopharmacology, 14(4), 253-263.

Shansis, F. M., Busnello, J. V., Quevedo, J., Forster, L., Young, S., Izquierdo, I., et al. (2000). Behavioural effects of acute tryptophan depletion in healthy male volunteers. $J$ Psychopharmacol, 14(2), 157-163.

Sheline, Y. I., Barch, D. M., Donnelly, J. M., Ollinger, J. M., Snyder, A. Z., \& Mintun, M. A. (2001). Increased amygdala response to masked emotional faces in depressed subjects resolves with antidepressant treatment: an fMRI study. Biological psychiatry, 50(9), 651658.

Siepmann, M., Grossmann, J., Muck Weymann, M., \& Kirch, W. (2003). Effects of sertraline on autonomic and cognitive functions in healthy volunteers. Psychopharmacology, 168(3), 293-298.

Siwek, D. F., \& Pandya, D. N. (1991). Prefrontal projections to the mediodorsal nucleus of the thalamus in the rhesus monkey. Journal of comparative neurology, 312(4), 509-524. 
Sobczak, S., Honig, A., Schmitt, J. A. J., \& Riedel, W. J. (2003). Pronounced cognitive deficts following an intravenous L-tryptophan challenge in first degree relatives of bipolar patients compared to healthy controls. Neuropsychopharmacology, 28, 711-719.

Sobczak, S., Riedel, W. J., Booij, L., Aan het Rot, M., Deutz, N. E. P., \& Honig, A. (2002). Cognition following Acute Tryptophan Depletion: differences between first-degree relatives of bipolar disorder patients and matched healthy control volunteers. Psychological Medicine, 32(3), 503-515.

Sogaard, B., Mengel, H., Rao, N., \& Larsen, F. (2005). The pharmacokinetics of escitalopram after oral and intravenous administration of single and multiple doses to healthy subjects. Journal of clinical pharmacology, 45(12), 1400-1406.

Soubrie, P. (1986). Reconciling the role of central serotonin neurons in human and animal behavior. Behavioral and Brain Sciences, 9, 319-364.

Spijker, J., Bijl, R. V., de Graaf, R., \& Nolen, W. A. (2001). Care utilization and outcome of DSMIII-R major depression in the general population. Results from the Netherlands Mental Health Survey and Incidence Study (NEMESIS). Acta psychiatrica Scandinavica, 104(1), 19-24.

Spoont, M. R. (1992). Modulatory role of serotonin in neural information processing: implications for human psychopathology. Psychological bulletin, 112(2), 330-350.

Stahl, S. M. (1998a). Basic psychopharmacology of antidepressants, part 1: Antidepressants have seven distinct mechanisms of action. Journal of Clinical Psychiatry, 59 Suppl 4, 514.

Stahl, S. M. (1998b). Mechanism of action of serotonin selective reuptake inhibitors. Serotonin receptors and pathways mediate therapeutic effects and side effects. Journal of Affective Disorders, 51(3), 215-235.

Steckler, T., \& Sahgal, A. (1995). The role of serotonergic-cholinergic interactions in the mediation of cognitive behaviour. Behavioural Brain Research, 67, 165-199.

Stordal, K. I., Lundervold, A. J., Egeland, J., Mykletun, A., Asbjornsen, A., Landro, N. I., et al. (2004). Impairments across executive functions in recurrent depression. Nordic Journal of Psychiatry, 58(1), 41-47.

Stroop, J. R. (1935). Studies of interference in serial verbal reactions. Journal of Experimental Psychology, 18, 643-662.

Sturm, W., \& Willmes, K. (2001). On the functional neuroanatomy of intrinsic and phasic alertness. NeuroImage, 14(1 Pt 2), S76-84. 
Swartz, J. R., Miller, B. L., Lesser, I. M., \& Darby, A. L. (1997). Frontotemporal dementia: Treatment response to serotonin selective reuptake inhibitors. Journal Of Clinical Psychiatry, 58(5), 212-216.

Talairach, J., \& Tournoux, P. (1988). Co-planar sereotaxic atlas of the human brain. New York: Thieme medical.

Teichner, W. H. (1974). The detection of a simple visual signal as a function of time of watch. Human factors, 16(4), 339-353.

Theunissen, E. L., Vermeeren, A., \& Ramaekers, J. G. (2006). Repeated-dose effects of mequitazine, cetirizine and dexchlorpheniramine on driving and psychomotor performance. British journal of clinical pharmacology, 61(1), 79-86.

Vaa, T. (2003). Impairments, diseases, age and their erlative risks of acident involvement: results from a meta-analysis. IMMORTAL-deliverable 1.1, Project funded by the European Commisison under the transport RTD Programme of the 5th framework programme.

Vermeeren, A., Jackson, J. L., Muntjewerff, N. D., Quint, P. J., Harrison, E. M., \& O'Hanlon, J. F. (1995). Comparison of acute alprazolam $(0.25,0.50$ and $1.0 \mathrm{mg})$ effects versus those of lorazepam $2 \mathrm{mg}$ and placebo on memory in healthy volunteers using laboratory and telephone tests. Psychopharmacology, 118(1), 1-9.

Vollenweider, F. X., Vollenweider Scherpenhuyzen, M. F., Babler, A., Vogel, H., \& Hell, D. (1998). Psilocybin induces schizophrenia-like psychosis in humans via a serotonin-2 agonist action. Neuroreport, 9(17), 3897-3902.

Vollm, B., Richardson, P., McKie, S., Elliott, R., Deakin, J. F., \& Anderson, I. M. (2006). Serotonergic modulation of neuronal responses to behavioural inhibition and reinforcing stimuli: an fMRI study in healthy volunteers. European journal of neuroscience, 23(2), 552-560.

Volz, H. P., Gaser, C., Hager, F., Rzanny, R., Mentzel, H., Kreitschmann-Andermahr, I., et al. (1997). Brain activation during cognitive stimulation with the Wisconsin Card Sorting Test - a functional MRI study on healthy volunteers and schizophrenics. Psychiatry Research, 75(3), 145-157.

Wechsler, D. (1981). The measurement and appraisal of adult intelligence. Baltimore: Williams and Wilkins.

WHO. (2001). The world health report 2001: mental health, new understanding, new hope.Unpublished manuscript. 
Wilkinson, C. J., Lee, G. S., Chang, C., \& Ventura, D. (2003). The effects of escitalopram administered alone and with alcohol on cognition and psychomotor performance. NIMH-43rd Annual NCDEU Meeting: poster session I.

Wingen, M., Bothmer, J., Langer, S., \& Ramaekers, J. G. (2005). Actual driving performance and psychomotor function in healthy subjects after acute and subchronic treatment with escitalopram, mirtazapine, and placebo: a crossover trial. Journal of clinical psychiatry, 66(4), 436-443.

Wingen, M., Kuypers, K. P. C., \& Ramaekers, J. G. (2007). The role of 5-HT1a and 5-HT2a receptors in attention and motor control: a mechanistic study in healthy volunteers. Psychopharmacology, 190, 391-400.

Wingen, M., Kuypers, K. P. C., \& Ramaekers, J. G. (2006b). Selective verbal and spatial memory impairment after 5-HT1a and 5-HT2a receptor blockade in healthy volunteers pretreated with an SSRI. Journal of Psychopharmacology, DOI:10.1177/0269881106072506.

Wingen, M., Langer, S., \& Ramaekers, J. G. (2006). Verbal memory performance during subchronic challenge with a selective serotonergic and a mixed action antidepressant. Human psychopharmacology, 21(7), 473-479.

Yasuno, F., Suhara, T., Nakayama, T., Ichimiya, T., Okubo, Y., Takano, A., et al. (2003). Inhibitory effect of hippocampal 5-HT1A receptors on human explicit memory. American journal of psychiatry, 160(2), 334-340.

Young, S. N. (1986). The clinical Psychopharmacology of Tryptophan. In R. J. Wurtman \& J. J. Wurtman (Eds.), Nutrition and the Brain (pp. 49-88). New York: Raven Press.

Young, S. N. (1996). Behavioral Effects of Dietary Neurotransmitter Precursors: Basic and Clinical Aspects. Neuroscience and Biobehavioral Reviews, 20(2), 313-323.

Young, S. N., \& Gauthier, S. (1981). Effect of tryptophan administration on tryptophan, 5hydroxyindoleacetic acid and indoleacetic acid in human lumbar and cisternal cerebrospinal fluid. J Neurol Neurosurg Psychiatry, 44(4), 323-327.

Young, S. N., \& Leyton, M. (2002). The role of serotonin in human mood and social interaction. Insight from altered tryptophan levels. Pharmacology Biochemistry and Behavior, 71, 857865.

Zakzanis, K. K., Leach, L., \& Kaplan, E. (1998). On the nature and pattern of neurocognitive function in major depressive disorder. Neuropsychiatry, neuropsychology, and behavioral neurology, 11(3), 111-119. 


\section{Summary}

The central theme in the present thesis is the behavioral toxicity of antidepressant drugs. It is hypothesized that the net action of antidepressants on serotonin neurotransmission has specific effects on cognition, including memory improvement and attention impairment, whereas other pharmacological mechanisms encountered in antidepressants, such as anticholinergic-, anti-adrenergic and antihistaminergic mechanisms may have a negative impact on performance.

The first part of the thesis concerns antidepressants that exert adverse effects on cognition and driving performance. The effects of specific antidepressant treatments on cognition and driving performance are investigated in healthy volunteers and depressed patients. The second part of the thesis concerns the role of serotonin in cognition. The neurotransmitter serotonin was manipulated by an antidepressant (particularly a selective serotonin reuptake inhibitor (SSRI)) in combination with 5-HT receptor agents and cognitive performance was assessed.

In Chapter $\mathbf{1}$ the aims of the present thesis are described. Research in animals as well as in humans demonstrates that serotonin is involved in different aspects of cognition including attention and memory. The question arises which pharmacological mechanisms mediate these effects of serotonin on memory and attention. Furthermore, serotonergic dysfunctioning is assumed to be part of the etiology of major depressive disorder and next to the more commonly recognised features as sadness and diminished energy, depression is often accompanied by a decline in cognitive and psychomotor functioning. Antidepressants are among the most prescribed psychoactive drugs nowadays of which SSRIs are the most common. Depending on the mechanism of action, antidepressant drugs exert different side effects, physically as well as cognitively. The aim of the present thesis was to assess the side effects of commonly used antidepressants on cognition and actual driving performance in healthy volunteers as well as in depressed patients. Expected was that treated depressed patients did not differ in cognitive function or driving performance compared to healthy volunteers because treatment with common prescribed antidepressants alleviates the depression with little adverse effect. In addition, the question was what the role of increased serotonin levels in memory and attention in humans is in different brain and receptor mechanisms. Manipulation of the $5-\mathrm{HT}_{1 \mathrm{~A}}$ and $5-\mathrm{HT}_{2 \mathrm{~A}}$ receptors was expected to augment the effects of pre-treated serotonin levels and by this means influencing memory and attention. Sustained attention impairment after SSRI treatment was expected to be observed in brain regions involved in sustained attention investigated in an fMRI study. 
In Chapter $\mathbf{2}$ an overview is given on experimental studies involving healthy human volunteers and selected (clinical) patients which revealed that manipulations of the central serotonin system can produce quite specific changes in cognitive functioning, independent of overt mood changes. Reduced serotonin turnover is consistently associated with impaired long-term memory functioning. Low serotonin function may also impair cognitive flexibility and improve focused attention. On the other hand, stimulation of central serotonin has repeatedly been found to impair performance in a true vigilance task. Currently, there is little evidence for mirrored cognitive changes due to opposite serotonin manipulations in healthy volunteers. Given the mounting evidence for a role of serotonin in human cognition, reduced serotonin function could be directly linked to cognitive disturbances in certain conditions, such as in depression and Alzheimer's Disease (AD). There is evidence that stimulating (i.e. normalizing) serotonin activity in depression may have specific beneficial effects on cognition, independent of a general relief of depressive symptoms, but this premise needs to be confirmed by larger-scale clinical studies. Recently, a potential role of serotonin in the cognitive symptoms in AD has been identified, but there is insufficient data to evaluate the effects of serotonin stimulation on cognitive symptoms in AD. It is concluded that serotonin is a potential target for pharmacological cognition enhancement, particularly for restoration of impaired cognitive performance due to serotonin dysfunction. Further differentiation of the role of serotonin in normal and disturbed cognition and evaluation of the effects of serotonin manipulations in various populations is required to establish the full potential of serotonin drugs as cognition enhancers.

In Chapter 3 the effects of escitalopram 10-20 mg per day and mirtazapine 30-45 mg per day on actual driving and psychomotor performance of 18 healthy subjects in a randomised, double-blind, placebo-controlled, multiple-dose, 3-way crossover trial are determined. The effects of ecitalopram on psychomotor and actual driving performance had not been investigated. Mirtazapine previously impaired psychomotor function and driving performance and in the present study it was included as an active control. It was expected that escitalopram had no effect but that mirtazapine would impair driving performance and psychomotor function. Each treatment period lasted for 15 days and was separated from the next period by a washout period of at least 13 days. Subjects received an evening dose of escitalopram $10 \mathrm{mg}$, mirtazapine 30mg, or placebo from Days 1-7 and an evening dose of escitalopram 20mg, mirtazapine $45 \mathrm{mg}$, or placebo from Days 8-15. On Days 2, 9, and 
16, reflecting acute period, dose increase, and steady state, respectively, the Road Tracking test was performed. The main parameter was Standard Deviation of Lateral Position. Psychomotor performance was also assessed on Days 2, 9, and 16 by laboratory computer tasks. Subjective sleep quality and mood were measured by visual analogue scales. Treatment differences were apparent during the acute treatment period, in which subjects treated with mirtazapine $30 \mathrm{mg}$ performed less well in the driving test as compared to placebo. The Divided Attention Task results also revealed a significant increase in tracking error after a single dose of mirtazapine $30 \mathrm{mg}$ as compared with placebo. Mirtazapine decreased feelings of alertness and contentedness. Escitalopram did not affect driving, psychomotor performance or subjective mood throughout treatment. In conclusion, driving performance, as well as psychomotor functioning, were not affected by escitalopram treatment in healthy subjects. Driving performance was significantly impaired after ingestion of mirtazapine $30 \mathrm{mg}$ during the acute treatment period.

In Chapter 4 actual driving performance and cognition of depressed patients receiving long-term antidepressant treatment is assessed. It is generally known that SSRIs have less impairing effects on driving performance than TCAs. Expected was that driving performance and cognition of depressed patients receiving long-term SSRI or SNRI treatment was comparable to that of healthy volunteers, due to alleviated depressive symptoms. Performance was assessed in 24 depressed patients receiving SSRI (Selective Serotonin Reuptake inhibitor) or SNRI (Serotonin and Noradrenalin Reuptake Inhibitor) treatment for 6-52 weeks and in 24 matched, healthy controls by means of two standardized on-the-road driving tests and laboratory tests of cognition. Data showed poorer driving performance as indicated by a higher Standard Deviation of Lateral Position (SDLP) or 'weaving motion' in medicated patients relative to controls. Time to speed adaptation and Critical Flicker Fusion Threshold were also impaired in medicated patients. Hamilton Depression Rating Scale scores in medicated patients were significantly higher as compared to controls. No other significant results between the two groups were demonstrated on the variables of the driving tests and laboratory tests of cognition. Depressed patients receiving long-term treatment with SSRI and SNRI type antidepressants show impaired driving performance. This impairment in driving performance can probably be attributed to residual depressive symptoms instead the of antidepressant treatment. 
In Chapter 5 the effects of escitalopram 10-20 mg per day and mirtazapine 30-45 mg per day on verbal memory of 18 healthy participants are assessed in a randomized, double-blind, placebo-controlled, 3-way crossover trial. It was expected that escitalopram may enhance verbal memory due to its serotonergic action and that mirtazapine would impair verbal memory due to its histaminergic affinity. Each treatment period lasted for 15 days and was separated from the next period by a washout period of at least 13 days. Participants received an evening dose of escitalopram $10 \mathrm{mg}$, mirtazapine $30 \mathrm{mg}$, or placebo from days 1-7 and an evening dose of escitalopram $20 \mathrm{mg}$, mirtazapine $45 \mathrm{mg}$, or placebo from days $8-15$. On days 2, 9, and 16 a visual verbal memory task was performed measusing drug effects during the acute phase, after dose increase and at steady state. Escitalopram did not affect immediate or delayed verbal memory score throughout treatment. During mirtazapine treatment, participants performed less well in the overall immediate recall score compared to placebo. This impairment was most pronounced in the final trial of the visual verbal learning task. Verbal memory was not affected by acute and subchronic escitalopram treatment in healthy participants. Overall immediate verbal memory was slightly but significantly impaired throughout mirtazapine treatment, probably due to a general reduction in overall arousal caused by $\mathrm{H}_{1}$ receptor blockade.

In Chapter $\mathbf{6}$ a study conducted according to a randomized, double-blind, placebo controlled, 4-way crossover design including 16 healthy volunteers, is described. Assessed was whether the $5-\mathrm{HT}_{1 \mathrm{~A}}$ and $5-\mathrm{HT}_{2 \mathrm{~A}}$ receptors are involved in the association between serotonin and memory. The treatment consisted of oral administration of escitalopram $20 \mathrm{mg}+$ placebo, escitalopram $20 \mathrm{mg}+$ ketanserin 50 $\mathrm{mg}$ (5- $\mathrm{HT}_{2 \mathrm{~A}}$ antagonist), escitalopram $20 \mathrm{mg}+$ pindolol $10 \mathrm{mg}$ (5- $\mathrm{HT}_{1 \mathrm{~A}}$ partial agonist) and placebo on 4 separate days with a wash-out period of minimum 7 days. It was expected that pindolol and ketanserin would augment the effect of escitalopram on memory. Different memory tasks were performed including task measuring verbal memory, spatial working memory and reversal learning. Escitalopram showed an impairing effect on immediate verbal recall which nearly reached statistical significance. No effects of escitalopram were found on other types of memory. In combination with pindolol, immediate verbal recall was significantly impaired. Escitalopram in combination with ketanserin impaired spatial working memory significantly. No effects were found on reversal learning. To conclude, selective impairment of immediate verbal recall after a $5-\mathrm{HT}_{1 \mathrm{~A}}$ partial agonist and selective impairment of spatial working memory performance after 5- $\mathrm{HT}_{2 \mathrm{~A}}$ receptor 
antagonist, both in combination with a selective serotonergic reuptake inhibitor (escitalopram), suggests that $5-\mathrm{HT}_{1 \mathrm{~A}}$ and $5-\mathrm{HT}_{2 \mathrm{~A}}$ receptors are distinctly involved in verbal and spatial memory.

In Chapter 7 the contribution of serotonin reuptake inhibition and specific blockade of the pre-synaptic $5-\mathrm{HT}_{1 \mathrm{~A}}$ receptor and the $5-\mathrm{HT}_{2 \mathrm{~A}}$ receptor to deficits in attention is assessed according to a randomized, double-blind, placebo controlled, 4-way crossover study design including 16 healthy volunteers. Treatments consisted of oral administration of the SSRI escitalopram $20 \mathrm{mg}+$ placebo, escitalopram $20 \mathrm{mg}+$ ketanserin (5- $\mathrm{HT}_{2 \mathrm{~A}}$ antagonist) $50 \mathrm{mg}$, escitalopram $20 \mathrm{mg}+$ pindolol $\left(5-\mathrm{HT}_{1 \mathrm{~A}}\right.$ antagonist) $10 \mathrm{mg}$ and placebo + placebo on 4 separate days. It was expected that pindolol and ketanserin would augment the impairing effect of escitalopram on sustained attention by acutely elevating serotonin levels and by decreasing dopamine release respectively. A range of performance tasks were conducted to assess the subjects' attention and motor functions. Escitalopram administered alone impaired tracking performance in a divided attention task. The combination of escitalopram and pindolol as well as escitalopram and ketanserin impaired divided attention as compared to placebo. In addition, escitalopram and ketanserin impared sustained attention. Divided attention impairment observed after combined treatments did not significantly differ from impairments after escitalopram alone. In conclusion, sustained attention impairment observed after combined escitalopram and ketanserin significantly differed from escitalopram alone. 5- $\mathrm{HT}_{1 \mathrm{~A}}$ receptor blockade hardly affected SSRI effects on attention. Additional 5- $\mathrm{HT}_{2 \mathrm{~A}}$ receptor blockade however produced impairments of sustained attention.

SSRIs were previously shown to impair sustained attention as measured with the Mackworth Clock Test. In Chapter $\mathbf{8}$ the influence of presumably increased serotonin levels on brain regions involved in sustained attention is examined by means of an fMRI study. Expected was that escitalopram administration would impair sustained attention observed in brain regions involved in sustained attention. Ten healthy volunteers $(5$ 이 $5 \hat{\jmath})$ received the selective serotonin reuptake inhibitor (SSRI) escitalopram (20mg) and placebo in a balanced, double blind, 2-way crossover design. Participants performed the Mackworth Clock Test to measure sustained attention during functional MRI measurements at 3-Tesla. Subjective measurements after pharmacological manipulation were conducted with the Bond \& Lader Questionnaire. Independent of treatment, brain areas associated with task performance on a sustained attention task were activated, including right prefrontal 
and parietal areas. After escitalopram administration, less activation was shown in the caudate nucleus, thalamus and frontal areas. No effect of escitalopram was shown on behavioral data although subjective measurements showed decreased alertness after escitalopram. The results suggest that serotonin stimulation impairs sustained attention through modulating activation of selective brain areas including the thalamus and caudate nucleus, which are possibly involved in a subcortical network for sustained attention.

In Chapter 9 the results of this thesis are discussed in a broader perspective. Overall, antidepressants with anticholinergic, anti-adrenergic or antihistaminergic mechanisms may impair cognition and driving performance in healthy subjects. It is very important to take into account any residual depressive symptoms by considering the effects of antidepressants on cognition and specifically driving performance in depressed patients. The research in this thesis gives additional evidence for an association between serotonin and memory because after administration of an SSRI in combination with a 5- $\mathrm{HT}_{1 \mathrm{~A}}$ partial agonist presumably increased serotonin levels impair verbal memory. Furthermore, it is also demonstrated that the $5-\mathrm{HT}_{2 \mathrm{~A}}$ receptor is an important receptor in modulating specific effects of serotonin on spatial memory and sustained attention. In addition, the effect of serotonin on an attentional network including the thalamus is possibly responsible for sustained attention performance. In this thesis, several relative new methods in human studies are introduced including the use of brain imaging and examining brain mechanisms at the receptor level. These methods could be further elaborated in future studies to acquire knew knowledge relating to neurotransmitter mechanisms, behaviour and the development of pharmacological treatment. 


\section{Samenvatting}

Het centrale thema van dit proefschrift is gedragstoxicologie van antidepressiva. De hypothese is dat de netto werking van antidepressiva op serotonine tot specifieke effecten leidt op cognitie, waaronder geheugen verbetering en aandacht verslechtering, en dat andere farmacologische mechanismen van antidepressiva waaronder anticholinerge, anti-adrenerge en antihistaminerge effecten een negatieve impact hebben op prestatie.

Het eerste gedeelte van dit proefschrift handelt over de bijwerkingen van antidepressiva op cognitie en rijvaardigheid. The effecten van bepaalde antidepressiva op cognitie en rijvaardigheid zijn gemeten bij gezonde vrijwilligers en depressieve patiënten. Het tweede gedeelte van het proefschrift handelt over de rol van serotonine in cognitieve prestaties. De neurotransmitter serotonine werd gemanipuleerd door de toediening van een selectief antidepressivum (in het bijzonder een selectieve serotonine heropname remmer (SSRI) al of niet in combinatie met middelen die op 5-HT receptoren werken terwijl cognitieve prestaties werden gemeten.

In hoofdstuk 1 worden de doelen van het huidige proefschrift besproken. Zowel dieronderzoek als humaanonderzoek laat zien dat serotonine betrokken is bij verschillende cognitieve aspecten waaronder aandacht en geheugen. De vraag is welke farmacologische mechanismen aan deze effecten van serotonine op geheugen en aandacht ten grondslag liggen. Daarbij wordt het disfunctioneren van serotonine gezien als een van de mogelijke oorzaken van een depressie en naast meer algemeen herkenbare kenmerken van depressie zoals neerslachtigheid en verlies van energie, gaat een depressie vaak gepaard met een afname van cognitief en psychomotorisch functioneren. Antidepressiva vallen momenteel onder de meest voorgeschreven psychoactieve medicatie en daarvan worden SSRIs het meest voorgeschreven. Antidepressiva verschillen in fysieke en cognitieve bijwerkingen, afhankelijk van het werkingsmechanisme. Het doel van het huidige proefschrift was het vaststellen van de bijwerkingen van vaak gebruikte antidepressiva op cognitie en rijvaardigheid bij zowel gezonde vrijwilligers als depressieve patiënten. Verwacht werd dat behandelde depressieve patiënten niet verschilden van gezonde vrijwilligers wat betreft cognitieve prestaties en rijvaardigheid omdat behandeling met veel voorgeschreven antidepressiva de depressie verlicht met weinig bijwerkingen. De vraag is ook wat de rol is van verhoogde serotonine niveaus in geheugen en aandacht bij mensen in verschillende hersen- en receptor mechanismen. Manipulatie van de 5$\mathrm{HT}_{1 \mathrm{~A}}$ en $5-\mathrm{HT}_{2 \mathrm{~A}}$ receptor werd verwacht de effecten van voorverhoogde serotonine 
niveaus te verergeren en daarbij geheugen en aandacht te beïnvloeden. Het werd verwacht dat verslechtering van volgehouden aandacht na SSRI toediening waar te nemen is in hersengebieden die betrokken zijn bij volgehouden aandacht, onderzocht in een $\mathrm{fMRI}$ onderzoek.

In hoofdstuk 2 wordt een overzicht gegeven van experimenteel onderzoek bij gezonde menselijke vrijwilligers en geselecteerde (klinische) patiënten waarin wordt besproken dat manipulaties van het serotonerge systeem specifieke veranderingen teweeg brengen in cognitief functioneren, onafhankelijk van zichtbare veranderingen in stemming. Verlaagde serotonine verwerking wordt herhaaldelijk geassocieerd met verslechterd lange termijn geheugen. Aan de andere kant is meerdere malen gebleken dat stimulatie van centrale sertonine de prestatie op een vigilantietaak verslechterd. Tot nu toe is er weinig bewijs voor gespiegelde cognitieve veranderingen door tegenovergestelde serotonine manipulaties bij gezonde vrijwilligers. Verlaagd serotonerg functioneren kan direct worden verbonden met cognitieve stoornissen bij bepaalde condities zoals depressie en de ziekte van Alzheimer (AD), gezien het groeiende bewijs voor een rol voor serotnine in menselijke cognitieve functies. Bewijs is geleverd dat stimulatie (oftewel normalisatie) van serotonerge activiteit bij depressie voordelige effecten kan hebben op cognitie, onafhankelijk van verlichting van de depressieve symptomen, alhoewel dit nog bevestigd moet worden door grootschalige klinische onderzoeken. Recent is er een potentiële rol voor serotonine in cognitieve symptomen bij AD vastgesteld, maar er zijn onvoldoende gegevens om de effecten van serotonine stimulatie op cognitieve symptomen bij AD te evalueren. Geconcludeerd kan worden dat serotonine een potentieel doel is voor farmacologische cognitieverbetering na serotonerg disfunctioneren. Verder onderscheid tussen de rol van serotonine in normaal en verstoord cognitief functioneren en evaluatie van de effecten van serotonerge manipulaties in verschillende populaties is vereist om de volwaardige rol van serotoninerge medicatie in cognitieverbetering vast te leggen.

In hoofdstuk 3 werden de effecten van escitalopram 10-20 mg per dag en mirtazapine 30-45 mg per dag onderzocht op rijvaardigheid en psychomotorisch functioneren bij 18 gezonde vrijwilligers in een gerandomiseerde, dubbelblinde, placebo gecontroleerde, 3 wegs onderzoeksprocedure. The effecten van escitalopram op psychomotorisch functioneren en rijvaardigheid waren nog niet eerder onderzocht. Eerder werd aangetoond dat mirtazapine psychomotorisch functioneren en rijvaardigheid verslechterd en in het huidige onderzoek werd het meegenomen als 
actief controlemiddel. Er werd verwacht dat escitalopram geen effect zou hebben maar dat mirtazapine rijvaardigheid en psychomotorisch functioneren negatief zou beïnvloeden. Iedere behandelingsperiode duurde 15 dagen en werd onderscheidden van de volgende periode door een uitwerkperiode van minimaal 13 dagen. Proefpersonen ontvingen een avonddosering escitalopram $10 \mathrm{mg}$, mirtazapine $30 \mathrm{mg}$ of placebo van dag $1 \mathrm{t} / \mathrm{m} 7$ en een avonddosering escitalopram $20 \mathrm{mg}$, mirtazapine 45 mg of placebo van dag $8 \mathrm{t} / \mathrm{m} \mathrm{15}$. Op dag 2, 9 en 16 dat wil zeggen in de acute periode, na verhoging van de dosering en in de stabiele staat werd de Road Tracking Test uitgevoerd. De hoofd parameter was de Standaard Deviatie van de Laterale Positie. Psychomotorisch functioneren werd ook gemeten op dag 2, 9 en 16. Subjectieve slaapkwaliteit en stemming werden door visuele analoge schalen gemeten. Verschillen tussen de medicatie werden zichtbaar in de acute periode waarin proefpersonen na mirtazapine $30 \mathrm{mg}$ slechter presteerden op rijvaardigheid vergeleken met placebo. De resultaten van de Divided Attention Task lieten en significante toename in tracking error zien na een enkele dosering van mirtazapine 30 mg vergeleken met placebo. Mirtazapine verminderde ook de alertheid en de tevredenheid. Escitalopram had geen effect op rijvaardigheid, psychomotorisch functioneren of subjectieve stemming tijdens de hele periode. Ter conclusie, zowel rijvaardigheid en psychomotorisch functioneren bij gezonde vrijwilligers werd niet beïnvloedt door escitalopram. Rijvaardigheid was significant slechter na inname van mirtazapine $30 \mathrm{mg}$ tijdens de acute behandelingsperiode.

In hoofdstuk 4 werden rijvaardigheid en cognitie van depressieve patiënten tijdens lange termijn behandeling met antidepressiva onderzocht. Het is bekend dat SSRIs in mindere mate een negatieve invloed hebben op rijvaardigheid dan TCAs. In het huidige onderzoek werd verwacht dat rijvaardigheid en cognitie van depressieve patiënten behandeld met SSRIs en een SNRI voor een langere termijn te vergelijken is met de rijvaardigheid en de cognitie van gezonde vrijwilligers, door de verlichting van depressieve symptomen. De prestatie van 24 depressieve patiënten behandeld met een SSRI of SNRI voor 6-52 weken, werd vergeleken met 24 gezonde vrijwilligers door middel van 2 gestandaardiseerde rijtesten en gedragstaken die cognitie meten. De resultaten lieten een slechtere rijvaardigheid zien ( grotere Standaard Deviatie van de Laterale Positie oftewel slingering) bij behandelde patiënten relatief ten opzichte van gezonden. De benodigde tijd om de snelheid aan te passen en de Critical Flicker Fusion drempelwaarde waren ook verslechterd bij behandelde patiënten. De scores op de Hamilton Depression Rating Scale waren bij behandelde patiënten significant hoger vergeleken met gezonden. Geen andere verschillen werden gemeten op het 
gebeid van rijvaardigheid en cognitie tussen de twee groepen. Geconcludeerd kan worden dat depressieve patiënten die voor een langere tijd behandeld worden met SSRIs of SNRIs een verslechterde rijvaardigheid laten zien. Deze verslechtering van rijvaardigheid is waarschijnlijk te wijten aan de nog steeds aanwezige depressieve symptomen en niet aan de behandeling met de genoemde antidepressiva.

In hoofdstuk 5 werden de effecten van escitalopram 10-20 mg per dag en mirtazapine 30-45 mg per dag onderzocht op verbaal geheugen bij 18 gezonde vrijwilligers in een gerandomiseerde, dubbelblinde, placebo gecontroleerde, 3 wegs onderzoeksprocedure. Er werd verwacht dat escitalopram verbaal geheugen verbeterd door de serotonerge werking en dat mirtazapine verbaal geheugen verslechterd door de histaminerge affiniteit. Proefpersonen ontvingen een avonddosering escitalopram $10 \mathrm{mg}$, mirtazapine $30 \mathrm{mg}$ of placebo van dag $1 \mathrm{t} / \mathrm{m} 7 \mathrm{en}$ een avonddosering escitalopram $20 \mathrm{mg}$, mirtazapine $45 \mathrm{mg}$ of placebo van dag $8 \mathrm{t} / \mathrm{m}$ 15. Op dag 2, 9 en 16 werd een verbale geheugen taak uitgevoerd, de effecten van de medicatie werden dus gemeten in de acute periode, na verhoging van de dosering en in de stabiele staat. Escitalopram had geen effect op direct of lange termijn verbaal geheugen tijdens de behandelingsperiode. Tijdens behandeling met mirtazapine presteerden de proefpersonen minder goed op de totale directe herhaling vergeleken met placebo. Deze verslechtering kwam het meeste naar voren in de laatste poging van de visuele verbale geheugen taak. Ter conclusie, verbaal geheugen werd niet beïnvloedt door acute en subchronische behandeling van escitalopram bij gezonde vrijwilligers. Totaal direct verbaal geheugen was enigszins maar significant verslechterd tijdens behandeling met mirtazapine en dit is waarschijnlijk te wijten aan afname van algemene alertheid veroorzaakt door $\mathrm{H}_{1}$ receptor blokkade.

Hoofdstuk 6 handelt over een gerandomiseerde, dubbelblinde, placebo gecontroleerde, 4-wegs studie met 16 gezonde vrijwilligers. Onderzocht werd of de 5$\mathrm{HT}_{1 \mathrm{~A}}$ en de $5-\mathrm{HT}_{2 \mathrm{~A}}$ receptor betrokken zijn in de associatie tussen serotonine en geheugen. De behandeling bestond uit de toediening van een capsule escitalopram 20 $\mathrm{mg}+$ placebo, escitalopram $20 \mathrm{mg}+$ ketanserine $50 \mathrm{mg}\left(5-\mathrm{HT}_{2 \mathrm{~A}}\right.$ antagonist), escitalopram $20 \mathrm{mg}$ + pindolol $10 \mathrm{mg}$ (5- $\mathrm{HT}_{1 \mathrm{~A}}$ partiële agonist) en placebo op 4 aparte dagen met een tussentijdse uitwerkperiode van minimaal 7 dagen. Verwacht werd dat pindolol en ketanserine het effect van escitalopram op geheugen zouden versterken. Verschillende geheugentaken werden uitgevoerd waaronder taken voor verbaal geheugen, ruimtelijk geheugen en reversal learning. $\mathrm{Na}$ escitalopram toediening was direct verbaal geheugen enigszins verslechterd, geen effecten van 
escitalopram werden gevonden op andere geheugentaken. In combinatie met pindolol was direct verbaal geheugen significant verslechterd. Escitalopram in combinatie met ketanserine verslechterde ruimtelijk werkgeheugen significant. Geen effecten werden gevonden op reversal learning. Selectieve verslechtering van direct verbaal geheugen na een $5-\mathrm{HT}_{1 \mathrm{~A}}$ receptor partial agonist en selectieve verslechtering van ruimtelijk werkgeheugen na een $5-\mathrm{HT}_{2 \mathrm{~A}}$ receptor antagonist, beide in combinatie met een SSRI (escitalopram) suggereert dat $5-\mathrm{HT}_{1 \mathrm{~A}}$ en $5-\mathrm{HT}_{2 \mathrm{~A}}$ receptors afzonderlijk betrokken zijn bij verbaal en ruimtelijk geheugen.

In hoofdstuk 7 werd de bijdrage van serotonine heropname inhibitie en specifieke blokkade van pre-synaptische $5-\mathrm{HT}_{1 \mathrm{~A}}$ receptors en de $5-\mathrm{HT}_{2 \mathrm{~A}}$ receptors aan stoornissen in aandacht onderzocht door middel van een gerandomiseerde, dubbelblinde, placebo gecontroleerde, 4 -wegs studie met 16 gezonde vrijwilligers. De behandeling bestond uit de toediening van een capsule escitalopram $20 \mathrm{mg}+$ placebo, escitalopram $20 \mathrm{mg}+$ ketanserine $50 \mathrm{mg}$ (5- $\mathrm{HT}_{2 \mathrm{~A}}$ antagonist), escitalopram $20 \mathrm{mg}+$ pindolol $10 \mathrm{mg}$ (5--HT $1 \mathrm{~A}$ partial agonist) en placebo op 4 aparte dagen met een tussentijdse uitwerkperiode van minimaal 7 dagen. Verwacht werd dat pindolol en ketanserine het verslechterende effect van escitalopram op volgehouden aandacht verergerden door respectievelijk acute verhoging van de serotonine niveaus en verlaging van dopamine afgifte. Om de aandachts en motorische functies van de proefpersonen te onderzoeken werd een reeks taken afgenomen. Na toediening van alleen escitalopram was tracking prestatie veslechterd in een verdeelde aandachtstaak. De combinatie escitalopram en pindolol en ook escitalopram en ketanserine verslechterden verdeelde aandacht. Daarbij verslechterde de combinatie escitalopram en ketanserine ook volgehouden aandacht. De verslechtering van verdeelde aandacht na de combinaties verschilden niet significant van escitalopram alleen. Geconcludeerd kan worden dat verlechtering van volgehouden aandacht na de combinatie escitalopram en ketanserine significant verschilt van alleen escitalopram toediening. Blokkade van de $5-\mathrm{HT}_{1 \mathrm{~A}}$ receptor beïnvloedde nauwelijks de effecten van een SSRI op aandacht. Blokkade van de $5-\mathrm{HT}_{2 \mathrm{~A}}$ receptor veroorzaakte echter wel verslechtering van volgehouden aandacht.

In eerder onderzoek werd aangetoond dat SSRIs volgehouden aandacht verslechteren gemeten met de Mackworth Clock Taak. In hoofdstuk 8 werd de invloed van vermoedelijk toegenomen serotonine niveaus onderzocht in hersengebieden betrokken bij volgehouden aandacht door middel van een fMRI onderzoek. Verwacht werd dat toediening van escitalopram volgehouden aandacht verslechterd en dat dit 
waarneembaar zou zijn in hersengebieden betrokken bij volgehouden aandacht. Tien gezonde vrijwilligers $(5 \circ, 5 \hat{\jmath})$ kregen de SSRI escitalopram (20 mg) toegediend en placebo in een gebalanceerde, dubbelblinde, 2-wegs studie. De Mackworth Clock Taak werd uitgevoerd in een 3-Tesla functionele MRI meting om volgehouden aandacht te meten. De Bond \& Lader vragenlijst werd afgenomen om subjectief de stemming te meten. Hersengebieden die geassocieerd zijn met taakprestatie op een volgehouden aandachtstaak waren geactiveerd, waaronder de rechter prefrontaal en pariëtale gebieden, onafhankelijk van medicatie. Na toediening van escitalopram was er een verminderde activiteit waarneembaar in de caudate nucleus en de thalamus en in frontale gebieden. De gedragsdata lieten geen effect van escitalopram zien hoewel subjectieve metingen lieten zien dat er een afname was van alertheid na escitalopram. Deze resultaten suggereren dat vermoedelijke serotonine stimulatie, volgehouden aandacht verslechterd door de activatie van specifieke hersengebieden te moduleren waaronder de thalamus en de caudate nucleus. Deze gebieden zijn mogelijk betrokken in een subcorticaal netwerk voor volgehouden aandacht.

In hoofdstuk 9 zijn de resultaten van dit proefschrift bediscussieerd in een breder perspectief. Over het algemeen hebben antidepressiva met anticholinerge, antiadrenerge of antihistamninerge mechanismen een negatief effect op cognitie en rijvaardigheid bij gezonde vrijwilligers. Als de effecten van antidepressiva op cognitie en rijvaardigheid bij depressieve patiënten worden beschouwd, is het belangrijk om rekening te houden met resterende depressieve symptomen na een behandeling. Het onderzoek in dit proefschrift geeft aanvullend bewijs voor een associatie tussen serotonine en geheugen door aan te tonen dat na toediening van een SSRI in combinatie met een $5-\mathrm{HT}_{1 \mathrm{~A}}$ partiële agonist verbaal geheugen verslechterd door vermoedelijk verhoogde serotonine niveaus. Bovendien is er ook aangetoond dat de $5-\mathrm{HT}_{2 \mathrm{~A}}$ receptor een belangrijke receptor is bij het moduleren van specifieke effecten van serotonine op ruimtelijk geheugen en volgehouden aandacht. Het effect van serotonine op een aandachtsnetwerk met daarin de thalamus is mogelijk verantwoordelijk voor de volgehouden aandachtsprestatie. In dit proefschrift zijn enkele relatief nieuwe methoden geïntroduceerd waaronder het gebruik van brain imaging en het onderzoeken van hersenmechanismen op receptor niveau. Deze methoden kunnen verder worden uitgebreid in toekomstig onderzoek om nieuwe kennis te verwerven over neurotransmitter mechanismen, gedrag en de ontwikkeling van medicijnen. 


\section{Dankwoord}

Aan het eind van dit proefschrift en dus ook aan het eind van een tijd waarin veel gebeurd en geleerd is, mag ik hier de mensen noemen die ik zeer dankbaar ben en waar ik veel om geef. Het proefschrift is gebouwd met de stenen die anderen mij hebben toegeworpen. Ik hoop dat jullie ook trots zijn op dit boekje!

Jan Ramaekers, als co-promotor, bedankt voor de kans die je me gegeven hebt om meer over psychofarmacologisch onderzoek te leren en bedankt voor je wijze inzichten. Als dagelijks begeleider, bedankt dat je deur altijd open stond en voor alle nuttige momenten op de werkvloer en tijdens congressen. Ik heb veel waardering voor je, ook voor je prestaties als hardloper.

Wim Riedel (promotor), bedankt voor je supervisie tijdens mijn hele aio-periode. Je wijze woorden en advies, soms van een afstand (Cambridge), soms van dichtbij (UM) hebben altijd goed geholpen, zeker in de laatste fase van dit proefschrift.

Jeroen Schmitt, bedankt voor je tijd, je suggesties en je royaliteit. Helaas (voor mij) verhuisde je naar Zwitserland, maar ook toen mocht ik je ieder moment storen voor tips en informatie. Ook al was het af en toe moeilijk als het commentaar van jullie begeleiders soms recht tegenover elkaar leek te staan, ik besef maar al te goed dat dit uiteindelijk voor vooruitgang heeft gezorgd.

Kim Kuypers, we hebben 4 jaar niet alleen onze kamer maar ook lief en leed gedeeld. Bedankt voor je steun, je grapjes en de mooie tijd! Gefeliciteerd met je super proefschrift, ik ben blij dat je mijn paranimf wilt zijn. Andere collega's, waaronder Eef Theunissen, bedankt dat ik altijd met vragen kon aankomen, maar ook Anke Sambeth, Tequilla-Tim Leufkens, Peter van Ruitenbeek, Anita van Oers, Annemiek Vermeeren, Petra Hurks, Sven Stapert, Jeroen van Deursen, Arjan Blokland, Erik Vuurman, Coene van Dijk, Pascal van Gerven, Caroline Lamers. Jullie bepaalden de plezierige werksfeer. Bedankt voor alle leuke en leerzame ervaringen tijdens onder andere EPU-vergaderingen, lunches, dagjes uit, borrels, kerstdiners of congressen.

Inez Ramaekers, Ananda Waelen en Heidi Lansdaal bedankt voor jullie gezelligheid, het carpoolen maakte de weg Heerlen-Maastricht en terug een stuk aangenamer. Inez, heel erg bedankt dat je mijn paranimf wilt zijn en me bijstaat bij het moment van de waarheid! 
Een speciaal bedankje voor de "fMRI-mensen", a special thanks to the "fMRIpeople". In particular, Elia Formisano, thank you for coordinating the fMRI study, for your inspiring words and thoughts. Vincent van de Ven, bedankt voor je hulp tijdens de data-analyse en voor de handige tips. Sven Gijsen, bedankt voor je geduld en gezelligheid bij het scannen van de proefpersonen.

Willy Jeurissen, Henk en Irma Brauers, Cees van Leeuwen, stagiaires en onderzoeksassistenten: Silke Conen, Nadine Kessen, Marije Meeuwissen, Michiel Schraven, Majken Klomp, Jolijn de Hoogh, allemaal bedankt voor jullie hulp bij de data-verzameling en voor jullie zeer gewaardeerde assistentie.

Zonder gegevens komt een aio nergens, daarom bedank ik hier in het bijzonder alle proefpersonen voor hun deelname!

Secretariaat neurocognitie, Annemie Jeukens en Riny Bodifee, bedankt voor de ondersteuning tijdens mijn aio-tijd en tijdens de laatste fase van het proefschrift.

En dan hier een bedankje aan alle familie en vrienden die buiten het werk voor ontspanning zorgden met mailtjes, leuke avonden en weekenden. In het bijzonder bedank ik Cher en Petra, oma Wingen en opa en oma Embrechts voor de interesse in mijn werkzaamheden.

Lieve papa en mama, bedankt voor jullie steun door dik en dun. Van jullie heb ik geleerd wat doorzetten is en voor jullie zal ik altijd mijn best blijven doen.

Lieve Monique, zussen bij toeval, vriendinnen naar keuze. Bedankt dat je er bent en altijd voor me klaarstaat! Benjamin, bedankt voor je Engelse hulpvaardigheid.

Als laatste maar als beste, lieve Raymond, de liefde die jij geeft, de rust die jij uitstraalt maar tegelijkertijd ook de energie die jij hebt, daar kan niemand tegenop. Jij bent alles voor mij! 


\section{Curriculum vitae}

Marleen Wingen werd geboren op 28 augustus 1980 te Heerlen. In 1998 behaalde ze het VWO diploma aan het Bernardinus college in Heerlen. Aansluitend startte Marleen met de studie Psychologie aan de universiteit Maastricht, afstudeerrichting Neuropsychologie. Ze studeerde af in 2003 na een jaar stage stage bij de afdeling medische Psychologie in het azM. In datzelfde jaar begon ze met promotie onderzoek op het gebied van de psychofarmacologie bij de faculteit der Psychologie aan de Universiteit Maastricht. Uiteindelijk heeft dit geresulteerd in het huidige proefschrift. Vanaf maart 2007 is Marleen Wingen werkzaam als statistisch onderzoeker bij het Centraal Bureau voor de Statistiek in Heerlen. Ook hoopt ze dit jaar de Master in Affecive Neuroscience af te ronden.

\section{Publications}

Wingen M, Bothmer J, Langer S, Ramaekers JG. (2005). Actual driving performance and psychomotor function in healthy subjects after acute and subchronic treatment with escitalopram, mirtazapine, and placebo: a crossover trial. Journal of clinical psychiatry, 66: 436-43

Wingen M, Ramaekers JG, Schmitt JAJ. (2006). Driving impairment in depressed patients receiving long-term antidepressant treatment. Psychopharmacology, 188: 84-91

Wingen M, Langer S, Ramaekers JG. (2006). Verbal memory performance during subchronic challenge with a selective serotonergic and a mixed action antidepressant. Human Psychopharmacology, 21:473-479

Wingen, M, Kuypers, KPC, Ramaekers, JG. (2006). Selective verbal and spatial memory impairment after 5-HT1a and 5-HT2a receptor blockade in healthy volunteers pretreated with an SSRI. Journal of Psychopharmacology, DOI:10.1177/0269881106072506

Wingen, M, Kuypers, KPC, Ramaekers, JG. (2007). The role of 5- $\mathrm{HT}_{1 \mathrm{a}}$ and 5- $\mathrm{HT}_{2 \mathrm{a}}$ receptors in attention and motor control: a mechanistic study in healthy volunteers. Psychopharmacology, 190: 391-400

Wingen, M, Kuypers, KPC, Ramaekers, JG. (2007). Sustained attention and serotonin: a pharmaco-fMRI study, submitted

Wingen, M, Ramaekers, JG en Schmitt, JAJ. (2007). Depressief en onder behandeling van antidepressiva: wat betekent dit voor de rijvaardigheid? Neuropraxis, 1, jaargang 11: $17-22$

Schmitt JAJ, Wingen M, Ramaekers JG, Evers EAT, Riedel WJ (2006). Serotonin and human cognitive performance. Current Pharmaceutical Design, 12: 2473-2486

Kuypers, KPC, Wingen, M, Samyn, N, Limbert, N, Ramaekers, JG. (2007). Acute effects of nocturnal doses of MDMA on measures of impulsivity and psychomotor performance throughout the night. Psychopharmacology, DOI 10.1007/s00213-006-0679-6

Kuypers, KPC, Wingen, M, Ramaekers, JG. (2007). Memory and mood during the night and in the morning after repeated evening doses of MDMA. Journal of Psychopharmacology, submitted 
